\title{
Infochemikalien von Stamm und Wurzel der Rot-Buche (Fagus sylvatica L.): Methodenentwicklung und Betrachtung des VOC-Musters
}

\author{
Dissertation \\ zur Erlangung des Doktorgrades \\ der Fakultät für Forstwissenschaften und Waldökologie \\ der Georg-August-Universität Göttingen
}

vorgelegt von

Christine Rachow

geboren in Hannover

Göttingen, den 01. Juli 2019 
1. Gutachter: Prof. Dr. Andreas Schuldt

2. Gutachter: Prof. Dr. Michael Müller

Tag der mündlichen Prüfung: 19. September 2019 
Für Clyde

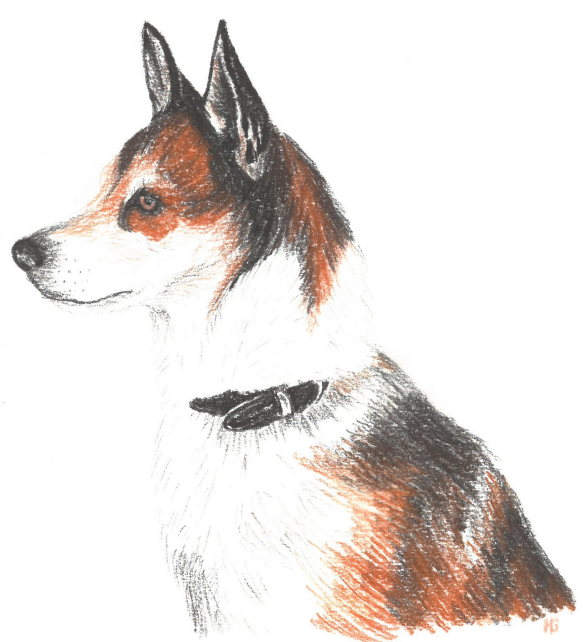




\section{Kurzfassung}

Ziel der Arbeit war es, Infochemikalien, die als Marker-VOC zur Früherkennung eines Insektenbefalls von durch Trockenheit geschwächten Bestände der Rot-Buche (Fagus sylvatica) dienen können, zu finden. Einige dieser VOC können von einigen Insekten als Hinweis genutzt werden, um gezielt gestresste und weniger gut verteidigte Bäume aufzusuchen.

Es wurden in dieser Arbeit zwei Messmethoden entwickelt, die es zum einen ermöglichen, das VOC-Muster von Stämmen im Freiland zu messen und zum anderen die Bodenbeweglichkeit von Wurzel-VOC nachzuweisen. Zusätzlich wurden VOC-Messungen an Stämmen wie auch an Wurzeln von F. sylvatica in Untersuchungsgebieten mit unterschiedlichen jährlichen Niederschlagsmengen durchgeführt und die Antennenreaktionen zwei an F. sylvatica vorkommenden Schmetterlingen (Calliteara pudibunda und Aglia tau) auf das Stamm-VOC-Muster von F. sylvatica untersucht.

Die Ergebnisse dieser Arbeit zeigen, dass sich das Stamm-VOC-Muster von F. sylvatica aus elf verschiedenen Stoffgruppen zusammensetzt, wobei vier Stoffgruppen (Terpene, Alkane, Aldehyde und Aromate) dominierten. Für das Wurzel-VOC-Muster konnten 42 VOC aus sieben Stoffgruppen identifiziert werden, wobei auch hier Terpene und Aldehyde dominierten. Ein Einfluss von unterschiedlichen jährlichen Niederschlagsmengen auf die Emissionsraten einzelner VOC konnte sowohl bei den Stamm- als auch bei den WurzelVOC-Mustern nachgewiesen werden.

Insgesamt konnten aus den Stamm- und den Wurzel-VOC-Mustern drei VOC ( $\alpha$-Pinen, Limonen und Nonanal) identifiziert werden, die als mögliche Marker-VOC genutzt werden können. Zwei dieser drei VOC (Limonen und Nonanal) lösten Antennenreaktionen bei $C$. pudibunda und A. tau aus, die für ihre Eiablage den Buchenstamm anfliegen. Alle drei VOC wurden in Untersuchungsgebieten mit jährlichen Niederschlagsmengen unter $600 \mathrm{~mm}$ in höheren Konzentrationen als in Gebieten mit jährlichen Niederschlagsmengen über $600 \mathrm{~mm}$ gemessen. Diese drei VOC sind bekannt dafür, entweder vermehrt bei Trockenstress ( $\alpha$-Pinen) oder bei Herbivorenfraß (Limonen und Nonanal) von Pflanzen emittiert zu werden und könnten als mögliche Marker für die VOC-basierte Früherkennung zur Abwehr von Schaderregern an F. sylvatica dienen.

In Zukunft könnten die gefundenen Marker-VOC helfen, gefährdete Bestände zu identifizieren, bevor sichtbare Schädigungen wie Blattverluste durch frühzeitigen trockenheitsbedingten Blattabwurf und/oder Insektenfraß eintreten. Dies würde das Auffinden möglicher Risikobestände erleichtern und helfen, schnellere Gegenmaßnahmen bei aufkommenden Kalamitäten durchzuführen. 


\section{Abstract}

Plant emission of volatile organic compounds (VOC) can be affected by changing environmental conditions such as temperature and drought. VOC play an important role in the interaction between plants and insects. For instance, insects use VOC, so-called infochemicals, to locate their host plants for feeding and oviposition. The aim of this study was to identify VOC released by drought stressed beech (Fagus sylvatica) trees. Some of these VOC can be a possible cue for certain insect species to reach stressed trees, which have a weakened defence against herbivore insects. Additionally, stress related VOC can act as an indicator for an early detection of insect infestation.

In this study, two methods were developed; one method to sample VOC emissions from stems of trees and the second one to investigate the soil mobility of VOC released by roots. Furthermore, stem as well as roots VOC patterns of drought stressed F. sylvatica under field conditions were collected at selected plots with different mean annual precipitations. Stem VOC patterns were measured over one year and root VOC patterns in spring and autumn.

The results of the study showed that VOC patterns from stems of F. sylvatica contained eleven different chemical groups, of which four chemical groups dominated (terpenes, alkanes, aldehydes and aromatic compounds). VOC pattern of roots contained seven different chemical groups, of which two chemical groups dominated (terpenes and aldehydes). Summarising the results, three VOC ( $\alpha$-pinene, limonene and nonanal) from both parts of the trees were identified as a potential VOC marker for plants under drought conditions ( $<600 \mathrm{~mm}$ mean annual precipitation). Additionally, this study indicates that VOC releasing rates of both stems and roots of $F$. sylvatica directly correlate to the annual precipitations.

In order to identify insect perceived VOC cues from stressed trees, stem VOC samples were tested with gas chromatography-electroantennographic detection (GC-EAD) for two different Lepidoptera species on beech (Calliteara pudibunda and Aglia tau). Two potential VOC markers (limonene and nonanal) eliciting antennal responses could be identified.

These experiments identified three important VOC, which are known to increase with decreasing annual precipitation. Two of these three VOC act as a kairomone for herbivores. These results combined with currently available pest management approaches can help to develop an early warning system for forest management implementation. 


\section{Danksagung}

Zunächst möchte ich mich bei Herr Prof. Dr. Andreas Schuldt und Herr Prof. Dr. Michael Müller für die Betreuung und die Begutachtung der Arbeit bedanken.

Vielen Dank an Anne für ihre schnelle Hilfe bei Fragen.

Ich möchte meinen Dank all jenen aussprechen, die mich in meiner Promotionszeit begleitet haben und mich aufgemuntert haben, wenn ich einmal nicht weiter wusste. In diesem Zusammenhang danke ich besonders den Mitarbeitern der Abteilung Forstzoologie und Waldschutz. Dabei möchte ich mich bei Sara Nicke-Mühlfeit für ihre Hilfe bei den Vor- und Nachbereitungen meiner Freilandversuche bedanken. Bei Dr. Martin Gabriel und Dr. Gerrit Holighaus möchte ich mich für die vielen regen und konstruktiven Gesprächsaustausche in den Pausen, bei Dr. Bernhard Weissbecker für seine Hilfe bei technischen Problemen und bei Frau Kistner für die Unterstützung mit Nervenfutter bedanken. Des Weiteren möchte ich mich bei Alisa Klamm und Katharina Gellrich für die gemeinsamen Arbeiten im Feld als auch im Labor bedanken.

Für das Korrekturlesen dieser Arbeit und die vielen hilfreichen Kommentare möchte ich mich an dieser Stelle bei meiner Schwester Karina, meinem Schwager Till, Nina Tiralla, Lena-Marie Simon, Katharina Gellrich, Karthi Balakrishnan, Christian Beck und Annett Reinhardt bedanken. Vielen Dank für die moralische Unterstützung und die Verpflegung in der Endphase. Ein besonderer Dank geht dabei an meine Arbeitskolleginnen Nina Tiralla und Lena-Marie Simon sowie an Katharina Gellrich, die mir bis zum Schluss Mut zugesprochen haben und mir nicht nur fachlich zur Seite standen, sondern mich in stressigen Momenten mit Kaffee und Schokolade versorgt haben. Ich möchte mich bei Dr. Carsten Thies für seine Hilfe bei statistischen Fragen und Niko Simon für seine Geduld bei Formatierungsfragen bedanken.

Mein tiefster Dank gilt meinen Eltern, die mir das Studium ermöglicht haben und mir bei meinen Entscheidungen immer zur Seite standen. Als guter Ratgeber hat mich Onkel Peter auf meinen bisherigen Lebensweg begleitet und stand mir mit meiner Familie als Rückhalt zur Verfügung.

Ein ganz herzlicher Dank geht an meine Schwestern und Brüder von Jomsborg, die mir die Stärke zum Durchhalten gegeben haben. „One shield, one brotherhood“.

Mein Entschluss Forstwissenschaften und Waldökologie zu studieren verdanke ich Frau Elke Mühlbach, bei der ich mich herzlich bedanken möchte.

Ein ganz besonderer und großer Dank gilt meinem vierbeinigen Freund Clyde, der mir stets ein treuer Gefährte war. Er hat mich bei meinen Freilandmessungen immer begleitet und auf unseren gemeinsamen Hunderunden für konstruktive Denkpausen gesorgt. Ruhe in Frieden Meister! 


\section{Inhaltsverzeichnis}

I. Kurzfassung

II. Abstract II

III. Danksagung III

\begin{tabular}{ll}
\hline IV. Abbildungsverzeichnis & VI
\end{tabular}

V. Tabellenverzeichnis $\quad$ IX

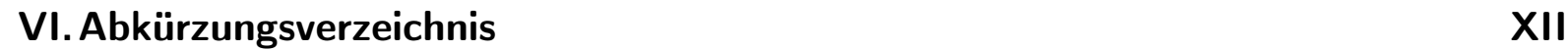

$\begin{array}{ll}\text { 1. Allgemeine Einleitung } & 1\end{array}$

2. Theoretischer Hintergrund 3

2.1. Klimafolgenforschung in Niedersachsen (KLIFF) . . . . . . . . . . . . . . 3

2.2. Flüchtige organische Verbindungen (VOC) . . . . . . . . . . . . . . 3

2.2.1. Pflanzen-Insekten-Interaktionen mittels VOC . . . . . . . . . . 3

2.2.2. VOC als Stressmarker . . . . . . . . . . . . . . . . . 5

2.3. Rot-Buche (Fagus sylvatica) . . . . . . . . . . . . . . . . 5

2.3.1. Vorkommen und Wuchsbedingungen . . . . . . . . . . 5

2.3.2. Trockenheit . . . . . . . . . . . . . . . . . . 6

2.4. Insekten an Rot-Buche (F. sylvatica $) \ldots \ldots \ldots$. . . . . . . . 7

2.5. Untersuchungsgebiete . . . . . . . . . . . . . . . . 8

2.6. Methodik der VOC-Analyse . . . . . . . . . . . . . . . . . . 12

2.6.1. Gaschromatographie . . . . . . . . . . . . . . . . 12

2.6.2. Massenspektrometrie . . . . . . . . . . . . . . . . 13

3. Stamm-Volatilenmuster von Rot-Buche (F. sylvatica) 15

3.1. Theoretischer Hintergrund ． . . . . . . . . . . . . . . . . . . . 17

3.1.1. Rot-Buchenstamm . . . . . . . . . . . . . 17

3.1.2. Oberirdische VOC-Muster von F. sylvatica . . . . . . . . . . . . . . 17

3.2. Ein neues Verfahren zum Sammeln von Stamm-VOC . . . . . . . . . . . . 19

3.2.1. Einleitung . . . . . . . . . . . . . . . . . . . 19

3.2.2. Material und Methoden . . . . . . . . . . . . . . 20

3.2.3. Ergebnisse . . . . . . . . . . . . . . . . . . . . 22

3.2.4. Diskussion . . . . . . . . . . . . . . . . . . . 24 
3.3. Kohlenstoffabgabe von Stämmen von F. sylvatica mittels VOC . . . . . . 26

3.3.1. Material und Methoden . . . . . . . . . . . . . 26

$3.3 .2 . \quad$ Ergebnisse . . . . . . . . . . . . . . . . . . 30

3.3.3. Diskussion . . . . . . . . . . . . . . . . . . . . . . . . 32

3.4. Stamm-VOC-Messung entlang eines Niederschlagsgradienten . . . . . . . . 34

3.4.1. Material und Methoden . . . . . . . . . . . . . . 34

$3.4 .2 . \quad$ Ergebnisse . . . . . . . . . . . . . . . . . . . . 38

3.4.3. Diskussion . . . . . . . . . . . . . . . . . . . . . . . . . . . . . . . . 49

3.5. Zusammenfassung . . . . . . . . . . . . . . . . . . 52

4. Wurzel-Volatilenmuster von Rot-Buche (F. sylvatica) 54

4.1. Theoretischer Hintergrund . . . . . . . . . . . . . . . . . . . . 56

4.1.1. Wurzelsystem von F. sylvatica . . . . . . . . . . . . . . . . . . . 56

4.1.2. VOC im Waldboden . . . . . . . . . . . . . . . . . . . . 58

4.1.3. Bisherige Methoden zur Messung von Wurzel-VOC . . . . . . . . . 62

4.1.4. Untersuchungsgebiete . . . . . . . . . . . . . . . . . 62

4.2. Wurzel-VOC-Messung . . . . . . . . . . . . . . . . . . . 63

4.2.1. Material und Methoden . . . . . . . . . . . . . 63

$4.2 .2 . \quad$ Ergebnisse . . . . . . . . . . . . . . . . . . 67

4.2 .3$. Diskussion . . . . . . . . . . . . . . . . . . . 75

4.3. Entwicklung einer Apparatur für die Untersuchung der Bodenbeweglichkeit von Wurzel-VOC . . . . . . . . . . . . . . . . . . . . . . . . . . . . . . . 80

4.3.1. Material und Methoden . . . . . . . . . . . . . . . . . . 81

$4.3 .2 . \quad$ Ergebnisse . . . . . . . . . . . . . . . . . . 93

4.3.3. Diskussion . . . . . . . . . . . . . . . . . . . . . . 101

4.4. Zusammenfassung . . . . . . . . . . . . . . . . . . . . . . 105

5. Olfaktorische Wahrnehmung von Insekten auf Volatile von $F$. sylvatica 108

5.1. Einleitung . . . . . . . . . . . . . . . . . . 109

5.2. Material und Methoden . . . . . . . . . . . . . . . . . . 109

5.3. Ergebnisse . . . . . . . . . . . . . . . . . . . . . . . . . 111

5.4. Diskussion . . . . . . . . . . . . . . . . . 111

\begin{tabular}{ll}
\hline 6. Gesamtdiskussion & 114
\end{tabular}

\begin{tabular}{lr}
\hline 7. Ausblick & 120
\end{tabular}

\begin{tabular}{ll}
\hline 8. Literaturverzeichnis & 121
\end{tabular} 
A. Anhang i

A.1. Laub-VOC von F. sylvatica . . . . . . . . . . . . . . . . i

A.2. Stamm-VOC von F. sylvatica . . . . . . . . . . . . . . . . . iv

A.3. Wurzel-VOC von F. sylvatica . . . . . . . . . . . . . . . . . . xix

A.4. Zuordnung der Wurzel-VOC von F. sylvatica zu weiteren Produzenten. . . xxii

A.5. Apparaturentwicklung für die Untersuchung der Bodenbeweglichkeit von

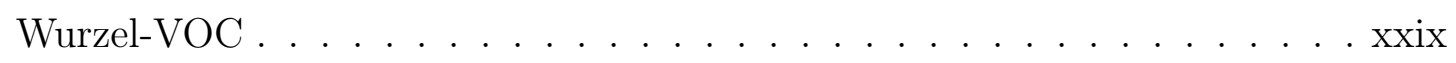

\section{Abbildungsverzeichnis}

1. Lage der Untersuchungsgebiete und Versuchsflächen (C) 2018 Google, erstellt mit QGis 3.6.2 ,Noosa“ for Mac OS X). . . . . . . . . . . . . . . . . . 10

2. $\quad$ Massenspektrum von (Z)-3-Hexen-1-ol (verändert nach der NIST Datenbank (MS Search 2.0)) . . . . . . . . . . . . . . . . 13

3. Darstellung eines Stammes von F. sylvatica . . . . . . . . . . . . . . . . . 14

4. $\quad$ Stammabsaugkammer a) Modell und b) Foto: 1: Probe (Tenax ${ }^{\circledR}$ TA), 2: Luftfilter $\left(\right.$ Tenax ${ }^{\circledR}$ TA oder CLSA), 3: Schnittstelle des Bratschlauches mit Leukoplast ${ }^{\circledR}$ fixiert, 4: Teflonadapter, 5: Spanngurt mit Ratschenverschluss, 6: Isomatte, 7: Teflonschlauch, 8: Bratschlauch, 9: Drehschieberpumpe . . . . . . . . . . . . . . . . . . . . . . . . . . 22

5. Total Ionenchromatogramm [TIC] der TDS-Proben im Jahr 2010 von $F$. sylvatica a) im August $\left(+20^{\circ} \mathrm{C}\right)$ und b) im November $\left(-1^{\circ} \mathrm{C}\right)$. Buchsta-

\begin{tabular}{|c|}
\hline ben: Identifizierung der Volatile mit dem Mass Spectral Search Library of \\
\hline the National Institute of Standards and Technology NIST (Gaithersburg,
\end{tabular} USA). a: 2-Methyl-1-pentanal; b: 2-Ethyl-3-methylbutanal; c: 3-Caren; d: p-Xylol; e: o-Xylol; f: Tricyclene; g: 2-Ethylhexanal Zahlen: Zusätzliche Identifizierung mit authentischen Standards. 1: Toluol; 2: Hexanal; 3: $\alpha$-Pinen; 4: Camphen; 5: $\beta$-Pinen; 6: Eukalyptol; 7: Tetradecan; 8: Pentadecan; 9: Hexadecan; 10: Heptadecan; 11: 2-Ethylhexanal . . . . . . . . . 23

6. Kohlenstoffabgabe von Stämmen von F. sylvatica. Hier dargestellt die Kohlenstoffabgabe (Mittelwerte \pm s. e.) von vier VOC bei einer zweistündigen Beprobung an dem jeweiligen Messtag im August, September, Oktober und November 2010 auf der Fläche Calvörde (Sand). Verschiedene Buchstaben geben signifikante Unterschiede in der Kohlenstoffabgabe zwischen den Messtagen wieder (Wilcoxon-Test mit Bonferroni-Korrektur, $\mathrm{p} \leq 0,013$ ) 30 
7. Kohlenstoffabgabe der vier VOC für die gesamte Stammoberfläche von $F$. sylvatica auf der Fläche Calvörde (Sand). Hier dargestellt die Mittelwerte aus sechs Messungen $(2 \mathrm{~h})$ der jeweiligen vier VOC . . . . . . . . . . . . . 31

8. Anzahl der VOC über das Jahr 2011 bei Stammmessungen von F. sylvatica im Untersuchungsgebiet Unterlüß aufgeteilt nach Stoffgruppen. $[S]=$ Sand,

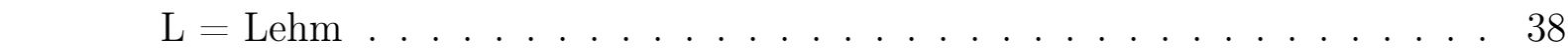

9. Anzahl der VOC über das Jahr 2011 bei Stammmessungen von F. sylvatica im Untersuchungsgebiet Göhrde aufgeteilt nach Stoffgruppen. S = Sand, L

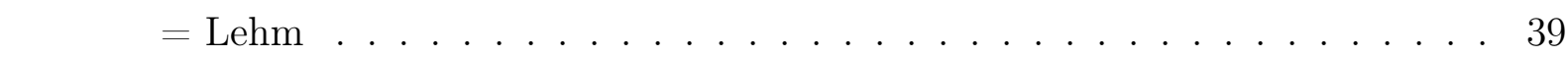

10. Anzahl der VOC über das Jahr 2011 bei Stammmessungen von F. sylvatica im Untersuchungsgebiet Calvörde aufgeteilt nach Stoffgruppen. S = Sand,

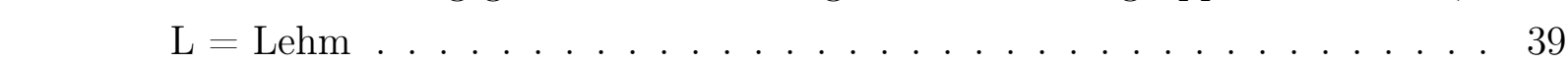

11. Vorkommen der Stoffklassen Aldehyd, Alkan, Alken, Alkohol und Aromat in allen genommenen Proben [\%] bei dem jeweiligen Untersuchungsgebiet, Bodentyp und Jahreszeit (Mittelwert \pm s. e.). Bonferroni-Test: ${ }^{*}=\mathrm{p}<$ $0,05,{ }^{* *}=\mathrm{p} \leq 0,01,{ }^{* * *}=\mathrm{p} \leq 0,001$. Un $=$ Unterlüß, Gö $=$ Göhrde, Ca

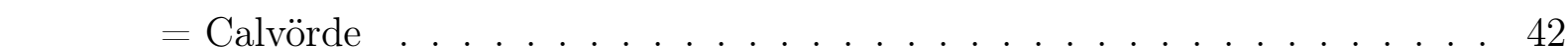

12. Vorkommen der Stoffklassen Ester, Keton, Terpen, Acetat und Unbekannt in allen genommenen Proben [\%] bei dem jeweiligen Untersuchungsgebiet, Bodentyp und Jahreszeit (Mittelwert \pm s. e.). Bonferroni-Test: ${ }^{*}=\mathrm{p} \leq$ $0,05,{ }^{* *}=\mathrm{p} \leq 0,01,{ }^{* * *}=\mathrm{p} \leq 0,001 . \mathrm{Un}=$ Unterlüß, Gö $=$ Göhrde, Ca $=$ Calvörde . . . . . . . . . . . . . . . . . . 44 43

13. Emittierte Konzentrationen von Nonanal und Decanal innerhalb von zwei Stunden über einen Stamm von F. sylvatica zu unterschiedlichen Jahreszeiten und jährlichen Niederschlagsmengen $\left(\bar{x} \pm\right.$ s. e.). Wilcoxon-Test: ${ }^{*}$ $=\mathrm{p} \leq 0,05,{ }^{* *}=\mathrm{p} \leq 0,01,{ }^{* * *}=\mathrm{p} \leq 0,001 . \mathrm{S}=$ Sand, $\mathrm{L}=$ Lehm, $1=$ Frühjahr, $2=$ Sommer, $3=$ Herbst, $4=$ Winter $\ldots \ldots \ldots$. . . . . 46

14. Emittierte Konzentrationen von $\alpha$-Pinen und Limonen innerhalb von zwei Stunden über einen Stamm von F. sylvatica zu unterschiedlichen Jahres\begin{tabular}{|c|}
\hline zeiten und jährlichen Niederschlagsmengen $(\bar{x} \pm$ s. e. $)$. Wilcoxon-Test: ${ }^{*}$ \\
\hline$=\mathrm{p} \leq 0,05,{ }^{* *}=\mathrm{p} \leq 0,01,{ }^{* * *}=\mathrm{p} \leq 0,001 . \mathrm{S}=$ Sand, $\mathrm{L}=$ Lehm, $1=$ \\
\hline
\end{tabular} Frühjahr, $2=$ Sommer, $3=$ Herbst, $4=$ Winter . . . . . . . . . . . . . 47

15. Emittierte Konzentrationen von Camphen und Campher innerhalb von zwei Stunden über einen Stamm von F. sylvatica zu unterschiedlichen Jah\begin{tabular}{l}
\hline reszeiten und jährlichen Niederschlagsmengen $(\bar{x} \pm$ s. e.). Wilcoxon-Test: \\
\hline$*=\mathrm{p} \leq 0,05,{ }^{* *}=\mathrm{p} \leq 0,01,{ }^{* * *}=\mathrm{p} \leq 0,001 . \mathrm{S}=$ Sand, $\mathrm{L}=$ Lehm, $1=$
\end{tabular} Frühjahr, $2=$ Sommer, $3=$ Herbst, $4=$ Winter . . . . . . . . . . . . . . . 48 
16. Schematische Darstellung eines Wurzelsystems . . . . . . . . . . . . . . . . 53

17. Schema der unterschiedlichen Mykorrhizatypen in Verbindung mit Baumwurzeln: Ekto- (links) und Endomykorrhiza (rechts) (verändert nach |1|)] . 57

18. $\quad$ Beispiel für Mikro- und Makrofauna in der Rhizosphäre . . . . . . . . . . . 58

19. Bedeutung von Terpenen bei der Interaktion von unterschiedlichen Organismengruppen in der Rhizosphäre. $+=$ positive Auswirkung; $-=$ negative

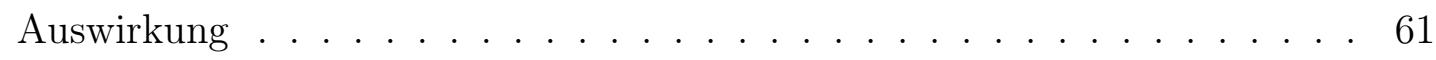

20. Bedeutung von Aldehyden bei der Interaktion von unterschiedlichen Organismengruppen in der Rhizosphäre. + = positive Auswirkung; - = negative

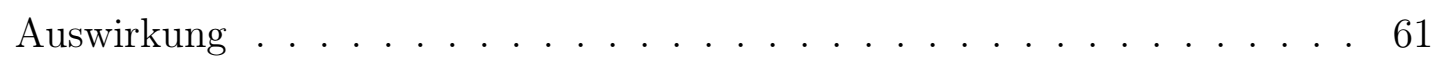

21. Versuchsaufbau zur Wurzel-VOC-Beprobung . . . . . . . . . . . . . . 65

22. Beispielchromatogramme von einer Wurzelprobe (a) und einer Stammprobe (b) von F. sylvatica. Die Zahlen stehen für verschiedene VOC: $1=$ Hexanal, $2=$ Heptanal, $3=\alpha$-Pinen, $4=$ Camphen, $5=2$-Ethylhexanal, $6=$ Benzaldehyd, $7=$ Octanal, $8=3$-Caren, $9=2$-Ethylhexanol, $10=$ Nonanal, TIC $=$ Totalionenstrom . . . . . . . . . . . . . . . . . . 69

23. Abhängigkeiten der Feinwurzel-VOC entlang des Niederschlagsgradienten.

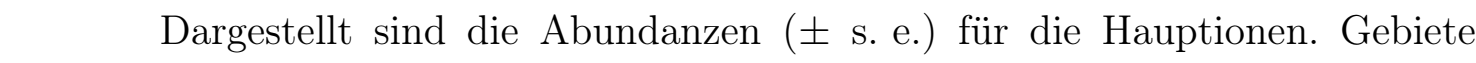
mit verschiedenen Buchstaben unterscheiden sich signifikant voneinander \begin{tabular}{|c|}
\hline (Wilcoxon-Test mit Bonferroni-Korrektur, $\mathrm{p} \leq 0,017)$. [SIM $=$ single ion \\
\hline
\end{tabular} modus . . . . . . . . . . . . . . . . . . 70

24. Signifikante saisonale Unterschiede in den Abundanzen ( \pm s. e.) der Hauptionen von ausgewählten VOC für Herbst (H, blau) und Frühling (F, rot). \begin{tabular}{|l|}
\hline Wilcoxon-Test: ${ }^{*}=\mathrm{p} \leq 0,05,{ }^{* *}=\mathrm{p} \leq 0,01,{ }^{* * *}=\mathrm{p} \leq 0,001 . \mathrm{S}=$ Sellhorn, \\
\hline
\end{tabular}

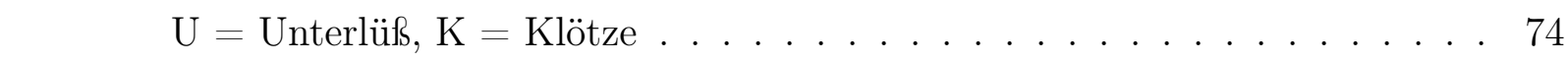

25. Zuordnung der gemessenen VOC zu (a) buchenbürtigen VOC und (b) mikrobiellen VOC (mVOC). . . . . . . . . . . . . . . . . . . 76

26. Apparatur mit Maßangaben ohne Teflonadapter . . . . . . . . . . . . . . 81

27. Aufbau der Apparatur mit eingedrehten Teflonadaptern und Markierung

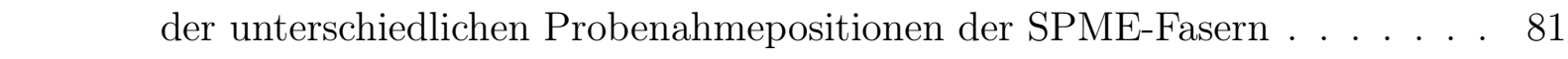

28. Seiten- und Frontansicht der Verschlusskappe . . . . . . . . . . . . . . . 82

29. Teflonadapter mit Metallgehäuse . . . . . . . . . . . . . . . . . . . 82

30. Querschnitt der Apparatur . . . . . . . . . . . . . . . . . . 83

31. Aufbau der Vorversuche (Foto: A. Klamm) . . . . . . . . . . . . . . . 86

32. SPME-Messung der Substratprobe in Pentan (Foto: A. Klamm) . . . . . . 90 
33. Chromatogramme der Kontrollmessungen des Probesubstrates (TIC = Totalionenstrom). Zahlen kennzeichnen die vier Hauptpeaks: 1 = Camphen, $2=$ Cyclotetrasiloxan, $3=$ Benzen, $4=\alpha$-Cedren . . . . . . . . . . . . . . 94

34. Nachweis von 3-Octanon in der Apparatur $10 \mathrm{~cm}$ links und rechts von der Duftquelle zu unterschiedlichen Messzeiten. Dargestellt ist die Peakfläche für das Ion 43. . . . . . . . . . . . . . . . . . . . . . . . 95

35. Nachweis von 3-Octanon in der Apparatur in 10, 30 und $50 \mathrm{~cm}$ Distanz zur Duftquelle zu unterschiedlichen Zeiten. Dargestellt ist die Peakfläche für das Ion 43. . . . . . . . . . . . . . . . . . . . . . . . . . 96 96

36. 1. Wiederholungsmessung mit 3-Octanon $\left(200 \mu l, 10^{-2}(\mathrm{w} / \mathrm{w})\right)$ in der Apparatur. Dargestellt sind die Peakflächen (Ion 43) bei den jeweiligen gemes\begin{tabular}{|c|}
\hline senen Distanzen zur Duftquelle bei den unterschiedlichen Messzeitpunkten \\
\hline
\end{tabular} in $\%$ zum Vergleichswert. . . . . . . . . . . . . . . . . . . . . . . . . 97

37. 2. Wiederholungsmessung mit 3-Octanon $\left(200 \mu l, 10^{-2}(\mathrm{w} / \mathrm{w})\right)$ in der Apparatur. Dargestellt sind die Peakflächen (Ion 43) bei den jeweiligen gemes\begin{tabular}{|c|}
\hline senen Distanzen zur Duftquelle bei den unterschiedlichen Messzeitpunkten \\
\hline
\end{tabular}

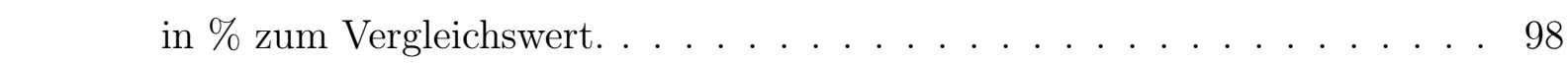

38. Calliteara pudibunda (on, links) und Aglia tau (o, rechts) . . . . . . . . . . 107

39. Sampling set up of bark volatiles . . . . . . . . . . . . . . . . 110

A.1. Feinwurzel mit Mykorrhizierung . . . . . . . . . . . . . . . . . . xix

A.2. Handgefertigte Skizze der Apparatur nach ersten Überlegungen . . . . . . xxix

A.3. Technische Zeichung der Apparatur und des Teflonadapters von A. Teichmann . . . . . . . . . . . . . . . . . . . . xxx

A.4. Technische Zeichnung des Deckels der Apparatur von A. Teichmann . . . . xxxi

\section{Tabellenverzeichnis}

1. Überblick der topographischen, klimatischen, edaphischen und bestandesstrukturellen Eigenschaften der untersuchten Buchenbestände entlang ei\begin{tabular}{|c|}
\hline nes Niederschlagsgradienten im norddeutschen Tiefland. (Quelle: Müller- \\
\hline
\end{tabular} Haubold et al. 2013 |2|, verändert) _. . . . . . . . . . . . . . . . . 11

2. $\quad$ Temperatur- und Luftfeuchtigkeitsdaten für den jeweiligen Messtag auf der

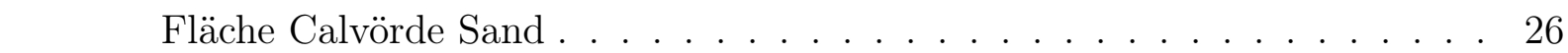

3. Temperatur und Luftfeuchtigkeit [\%] an den Messtagen . . . . . . . . . . . 35

4. Signifikante Unterschiede hinsichtlich des Vorkommens der Stoffklassen in

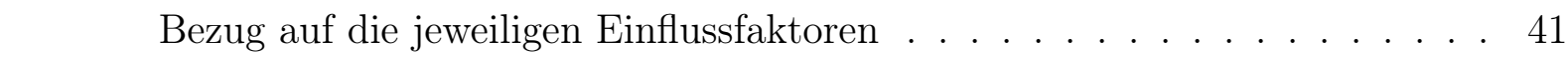


5. Jahreszeitliche Konzentrationsveränderungen von Nonanal und Decanal für den jeweiligen Bodentyp . . . . . . . . . . . . . . . . . . 46

6. Jahreszeitliche Konzentrationsveränderungen von $\alpha$-Pinen und Limonen für den jeweiligen Bodentyp . . . . . . . . . . . . . . . . . . . . . 47

7. Jahreszeitliche Konzentrationsveränderungen von Camphen und Campher für den jeweiligen Bodentyp . . . . . . . . . . . . . . . . . . . . . 48

8. Häufigkeiten der identifizierten Feinwurzel-VOC . . . . . . . . . . . . . . . 68

9. Signifikante Unterschiede in den VOC-Abundanzen des Hauptiones zwischen den Untersuchungsgebieten (Wilcoxon-Test mit Bonferroni-Korrektur,

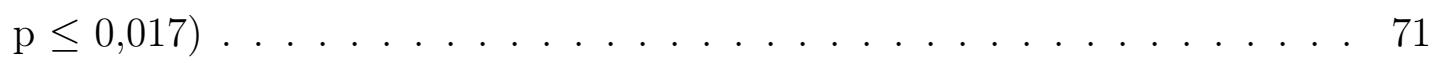

10. Häufigkeiten der identifizierten Feinwurzel-VOC für Herbst und Frühling . 73

11. Signifikante saisonale Unterschiede in den VOC-Häufigkeiten der Untersuchungsgebiete (Exakter Fisher-Test, $\mathrm{p} \leq 0,05)$. . . . . . . . . . . . . . . . 74

12. Chemische, physikalische und allgemeine Eigenschaften der VOC, die bei der Erprobung der Apparatur verwendet wurden . . . . . . . . . . . . . . . 84

13. Übersicht der Vorversuche . . . . . . . . . . . . . . . . . . . . 86

14. Peakfläche für das Hauption 43 beider Messreihenfolgen der Faserkalibrierung 93

15. Peakflächen des Hauptions (Ion 43) für 2-Heptanon und 3-Octanon bei den

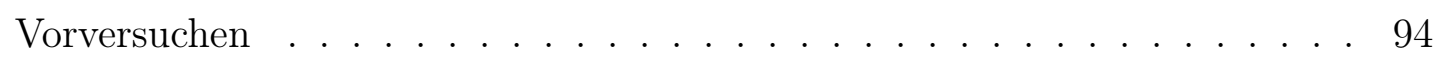

16. Vergleichswert für die Startmenge an der Startposition zum Zeitpunkt t $=096$

17. Halbmaxima und die dazugehörigen interpolierten Zeitpunkte für die erste \begin{tabular}{|c|}
\hline Wiederholungsmessung im Hauptversuch mit 3-Octanon. Peakfläche ermit- \\
\hline
\end{tabular}

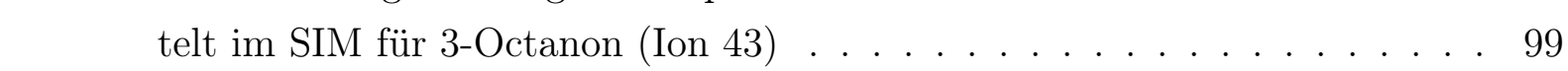

18. Halbmaxima und die dazugehörigen interpolierten Zeitpunkte für die zweite Wiederholungsmessung im Hauptversuch mit 3-Octanon. Peakfläche ermittelt im SIM für 3-Octanon (Ion 43) . . . . . . . . . . . . . . . . . . . . 99

19. Diffusionskoeffizienten für beide Wiederholungsmessungen mit 3-Octanon . 99

20. Diffusionskonstante für beide Wiederholungsmessungen mit 3-Octanon . . 100

21. SPME-Proben des Sand/Pentan-Gemisches . . . . . . . . . . . . . . . . 100

22. Dichlormethan/Methanol-Proben des Sand/Pentan-Gemisches . . . . . . . 100

23. Retention index, compound names and number of responding antennae from females and males of Calliteara pudibunda to different beech bark volatiles . . . . . . . . . . . . . . . . . . . 111

24. Retention Index, Compound names and number of responding antennae from females and males of Aglia tau to different beech bark volatiles. . . . 112

A.1. Bekannte VOC von Zweigen und Blätter von F. sylvatica in der Literatur . i

A.2. Stamm-VOC des Untersuchungsgebiet Unterlüßs. . . . . . . . . . . . . . v v 
A.3. Stamm-VOC des Untersuchungsgebiet Göhrde. . . . . . . . . . . . . . . . ix

A.4. Stamm-VOC des Untersuchungsgebiet Calvörde. . . . . . . . . . . . . . . . xiii

A.5. Signifikante Unterschiede in den Konzentrationen verschiedener VOC in Bezug auf die Einflussfaktoren . . . . . . . . . . . . . . . . . xviii

A.6. Signifikante Abhängigkeit der Konzentrationen von sechs VOC bei drei Einflussfaktoren . . . . . . . . . . . . . . . . . . xviii

A.7. Klimadaten für die Wurzel-VOC-Messungen im Mai . . . . . . . . . . . . . xix

A.8. Wurzellänge der Feinwurzeln im Oktober 2013 . . . . . . . . . . . . . . . . xx

A.9. Wurzellänge, Abstand zum jeweiligen Baum und BHD für die VOC-Messung der Feinwurzeln im Mai 2015. . . . . . . . . . . . . . . . . . . . . . xxi

A.10.Messplan des zweiten Hauptversuches mit 3-Octanol: 1. Wiederholung in der Apparatur 1 . . . . . . . . . . . . . . . . xxxii

A.11.Messplan des zweiten Hauptversuches mit 3-Octanol: 2. Wiederholung in der Apparatur 2 . . . . . . . . . . . . . . . . . xxxiii

A.12.Identifizierte VOC der Kontrollmessungen vom Probesubstrat . . . . . . . xxxiii

A.13.Identifizierte VOC der Kontrollmessungen von der leeren Apparatur . . . . xxxiv

A.14.Identifizierte VOC der Kontrollmessungen von der Apparatur mit eingefülltem Probesubstrat . . . . . . . . . . . . . . . . . . . xxxiv

A.15.Peakfläche (SIM: Ion 43) zu den Untersuchungen mit 3-Octanon bei $10 \mathrm{~cm}$ links und rechts von der Startposition . . . . . . . . . . . . . . xxxv

A.16.Peakfläche (SIM: Ion 43) zu den Untersuchungen mit 3-Octanon bei den Distanzen 10, 30 und $50 \mathrm{~cm}$ rechts von der Startposition . . . . . . . . . . xxxv

A.17.Peakflächen von 3-Octanon (SIM: Ion 43) für den Hauptversuch Teil 2. . . xxxvi 


\section{Abkürzungsverzeichnis}

AM arbuskuläre Mykorrhizapilze

ANOVA Varianzanalyse (analysis of variance)

BHD Brusthöhendurchmesser

C Kohlenstoff

CAS Registernummer (chemical abstracts service number)

CLSA closed-loop stripping analysis

EAG Electroantennographie

GC Gaschromatograph

GC-MS Gaschromatograph gekoppelt mit einem Massenspektrometer

GC-MS/EAD GC-MS mit einem elektoantennographischen Detektor

h Stunde

GLV Grünblattdüfte (green leaf volatiles)

K Kelvin

KLIFF Klimafolgenforschung in Niedersachsen

kPa Kilopascal

L Lehm

LD letale Dosis

LRI linearer Retentionsindex

Max. Maxima

Mio. Millionen

Mrd. Milliarden

MT Monoterpene

MS Massenspektrometer 
$\mathbf{m} / \mathbf{z} \quad$ Masse/Ladungs-Verhältnis

N Anzahl

n. a. nicht abrufbar

NIST National Institute of Standards and Technology

n. s. nicht signifikant

nWSK nutzbare Wasserspeicherkapazität

RI Retentionsindex

RT Retentionszeit

S Sand

s. e. $\quad$ Standardfehler

SIM single ion modus

SPME Festphasenmikroextraktion (solid phase microextraction)

ST Sesquiterpene

TDS Thermodesorption System (thermal desorption system)

TIC Totalionenstrom (total ion current)

Tsd. Tausend

VOC flüchtige organische Verbindungen (volatile organic compounds)

$\mathbf{w} / \mathbf{w} \quad$ weight/weight

WRB world reference base for soil recources 


\section{Allgemeine Einleitung}

Durch die Zunahme von extremen Wetterbedingungen, verursacht durch den Klimawandel [3], werden sich abiotische Umweltbedingungen wie die Wasserversorgung von Waldstandorten in der Zukunft verändern. Bis zum Ende des 21. Jahrhunderts wird mit einer globalen Zunahme der Temperatur zwischen 1,5 und 2 Grad gerechnet [3]. Die Sommermonate im kontinental geprägten Europa werden voraussichtlich trockener werden und Niederschlagsunterschiede zwischen feuchten und trockeneren Regionen werden zunehmen [3]. Steigende Temperaturen und infolgedessen hohe Transpirationen in Kombination mit saisonal knapper Wasserverfügbarkeit kann Sommertrockenheit zu einem der Hauptprobleme für wichtige Hauptbaumarten wie der Rot-Buche (Fagus sylvatica) machen [4] und die Stressanfälligkeit von Bäumen erhöhen [5].

Die sich verändernden Umweltbedingungen, etwa sich häufende und in der Stärke zunehmende Trockenheitsereignisse während der Vegetationsperiode, wie es in den Jahren 2003 und 2018 der Fall war, führen bei F. sylvatica zu einer möglichen Beeinträchtigung der Konkurrenzfähigkeit [6,7] sowie der Vitalität und Produktivität [8 aufgrund ihrer Sensibilität gegenüber geringer Wasserverfügbarkeit [6] und länger anhaltenden Trockenperioden während der Vegetationsperiode 911 .

Nicht nur die Vitalität, sondern auch die Anfälligkeit der Bäume gegenüber Insekten wird beeinflusst. Wärmeliebende Insekten können von den sich verändernden Umweltbedingungen profitieren [5, 12], weshalb mit vermehrten Gradationen von Forstschadinsekten zu rechnen ist 5, 12,13. . Doch nicht nur sich verändernde Umweltbedingungen wirken sich positiv auf diese Insekten aus, auch durch Trockenheit gestresste Pflanzen sind physiologisch besser für Insekten als Wirtspflanzen geeignet, da hier die Pflanzennährstoffe konzentrierter vorliegen [14]. Folglich sind solche Pflanzen gute Nahrungsquellen, die das Wachstum, das Überleben und die Reproduktion vor allem der rinden- und holzbrütender Insekten verbessern 15.

Mittels flüchtiger organischer Verbindungen (volatile organic compounds, VOC), die bei der Interaktion zwischen Insekten und Pflanzen als Signal- und Botenstoffe dienen [16], auch Infochemikalien genannt [17], können Insekten jene Wirtspflanzen finden, die eine gute Entwicklung der Nachkommen gewährleisten [18. Es kann daher angenommen werden, dass herbivore Insekten, die durch Trockenstress veränderte VOC-Muster von Pflanzen wahrnehmen können [19, 20, bestimmte Infochemikalien nutzen, um zwischen gut und weniger gut verteidigten Pflanzen zu selektieren [21.

Aufgrund dessen ist es wichtig frühzeitig zu erkennen, wann ein Bestand durch Trockenheit geschwächt ist, bevor die sichtbaren Anzeichen (wie z. B. frühzeitiger Laubabwurf |9|) auftreten und es zu Gradationen von Forstschadinsekten kommt, damit rechtzeitig Maß- 
nahmen gegen diese eingeleitet werden können. Das Messen von Infochemikalien bietet eine Möglichkeit, um den Stresszustand von Pflanzen zu ermitteln [22, 23. Karl et al. (2008) wiesen nach, dass Methylsalicylat im Luftraum von Kronen als ein frühes chemisches Warnsignal zur Diagnose des Waldstresses verwendet werden kann, bevor sichtbare Schäden an den Waldbäumen erkennbar sind [24]. Auch bei jungen Pflanzen von F. sylvatica konnte eine Veränderung der Abgabe von Monoterpenen (MT) bei unterschiedlichen Trockenstressstärken nachgewiesen werden 25].

Bisherige Studien, die sich mit dem VOC-Muster von trocken-gestressten Baumarten auseinandergesetzt haben, wurden an jungen Pflanzen durchgeführt [26 29]. Es gibt eine Untersuchung zum VOC-Muster von trocken-gestressten Blättern von F. sylvatica 25. Zum Stamm- und Wurzel-VOC-Muster von ausgewachsenen trocken-gestressten Bäumen im Freiland ist dagegen noch nichts bekannt.

Diese Arbeit beschäftigt sich daher mit dem VOC-Muster vom Stamm als auch von der Wurzel von F. sylvatica auf Standorten mit unterschiedlichen jährlichen Niederschlagsmengen und war Teil des Verbundprojektes „KLIFF“ (Klimafolgenforschung in Niedersachsen), welches vom niedersächsischen Ministerium für Wissenschaft und Kultur gefördert wurde.

Das Gesamtziel dieser Studie ist es herauszufinden, ob es für trocken-gestresste Bestände von F. sylvatica Marker-VOC gibt, die auf einen bevorstehenden Befall mit Schadinsekten hinweisen können. Diese Hauptfragestellung soll anhand folgender Hypothesen geklärt werden:

Hypothese 1: Das Stamm-VOC-Muster von F. sylvatica ändert sich auf Standorten entlang eines Niederschlagsgradienten (s. Kapitel 3).

Hypothese 2: Das Wurzel-VOC-Muster von F. sylvatica ändert sich auf Standorten entlang eines Niederschlagsgradienten (s. Kapitel 4).

Hypothese 3: Es können Antennenreaktionen von zwei an F. sylvatica vorkommenden Schmetterlingen (Calliteara pudibunda und Aglia tau) auf einzelne VOC des Stamm-VOCMuster von F. sylvatica gemessen werden (s. Kapitel 5).

Im folgenden Kapitel 2 wird der theoretische Hintergrund, der sich auf die Gesamtstudie bezieht, erläutert. 


\section{Theoretischer Hintergrund}

\subsection{Klimafolgenforschung in Niedersachsen (KLIFF)}

KLIFF ist ein interdisziplinärer Forschungsverbund, an dem 25 Universitäten und Forschungseinrichtungen aus den Bereichen Agrar- und Forstwissenschaften, Biologie, Geographie, Ingenieurwissenschaften, Meeresforschung, Meteorologie, Raumplanung, Sozialwissenschaften und Wirtschaftswissenschaften zusammengearbeitet haben, um eine Wissensgrundlage über die Auswirkungen des Klimawandels zu schaffen und um praktikable Anpassungsstrategien für die Folgen des Klimawandels zu erarbeiten. Das Verbundprojekt hatte eine Laufzeit von fünf Jahren (Januar 2009 bis Dezember 2013) und wurde vom niedersächsischen Ministerium für Wissenschaft und Kultur (MWK) gefördert [30].

Es wurde in diesem Verbundprojekt nach Lösungsansätzen für die Region Niedersachsen gesucht, in dessen Zentrum dabei die Wirtschaftszweige Landwirtschaft, Forstwirtschaft und Wasserwirtschaft sowie der Küstenschutz standen. Als Modellregionen wurden der Küstenraum, das Aller-Leine-Einzugsgebiet, der Harz und die Heide ausgewählt [30]. Im Forschungsthema Wald, eines der sieben Forschungsthemen von KLIFF, wurde der Fragestellung nachgegangen, wie sich der Klimawandel auf die Hauptbaumarten Rot-Buche (F. sylvatica) und Fichte (Picea abies) auswirkt 30.

\subsection{Flüchtige organische Verbindungen (VOC)}

Als flüchtige organische Verbindung (volatile organic compounds = VOC) wird jede organische Verbindung benannt, die bei 293,15 K einen Dampfdruck von 0,01 $\mathrm{kPa}$ oder mehr hat 31.

Pflanzen produzieren eine große Anzahl dieser VOC, von denen inzwischen über 1.700 von den verschiedenen Pflanzenfamilien der Angio- und Gymnospermen identifiziert wurden [32. Die physikalische Eigenschaft der VOC, bereits bei Raumtemperatur als Gas vorzuliegen, ermöglichen es diesen sich frei durch die Zellmembranen der Pflanzen zu bewegen und können so von den Pflanzen in die Umgebungsluft abgegeben werden 33.

Die Haupt-Biosynthesewege der pflanzlichen VOC sind der Shikimisäureweg, der Mevalonatweg, der Methylerythritolphosphatweg und die Lipoxygenase. Diese Synthesewege führen zur Bildung von benzenoiden und phenylpropanoiden VOC, Terpenen und Fettsäurederivaten (wie z. B. Grünblattdüfte, GLV] 34].

\subsubsection{Pflanzen-Insekten-Interaktionen mittels VOC}

Die als Infochemikalien fungierenden VOC spielen eine wichtige Rolle in der ökologischen Kommunikation von Pflanzen und Insekten und dienen der inter- und intras- 
pezifischen Kommunikation. So wurden Interaktionen zwischen Pflanzen [35, 36], Insekten [37,38] und zwischen Pflanzen und Insekten [39| bereits gut beschrieben.

Jede Pflanze hat ihr charakteristisches VOC-Muster, welches durch unterschiedliche abiotische und biotische Faktoren beeinflusst und verändert werden kann. Abiotische Umweltbedingungen wie z. B. Wasserverfügbarkeit, Licht und Temperatur können Pflanzen dazu veranlassen, unterschiedliche VOC zu emittieren [40,41. Ebenso kann Herbivorenfraß das VOC-Muster von Pflanzen wie von F. sylvatica beeinflussen 39] und die Emission von VOC aus unterschiedlichen chemischen Stoffgruppen wie z. B. Terpene (Campher, Eukalyptol), Ester (Methylsalicylat) und Aldehyde (Nonanal) bei befallenen Pflanzen hervorrufen 42,44 .

Durch Herbivorenfraß induzierte pflanzliche VOC können sowohl intra- als auch interspezifisch als Signale für Pflanzen dienen und zur Vorbereitung und Induzierung von Pflanzenabwehrmechanismen führen [45 47]. Dabei sind vorwiegend Terpene und GLV an der indirekten Pflanzenverteidigung beteiligt [48 50]. Von verletzten Pflanzen abgegebenes (Z)-3-Hexen-1-ol kann z. B. bei unbefallenen Nachbarpflanzen eine vorbeugende Verteidigung auslösen [16,51]. In vielen Fällen helfen VOC, die von Blättern durch Insektenfraß abgegeben werden, den Antagonisten von Herbivoren zwischen befallener und unbefallener Pflanze zu unterscheiden [52,54].

Die Emission von Pflanzen-VOC kann unterschiedliche Verhaltensreaktionen bei den Insekten bewirken, welche von Art zu Art [55,56] und zwischen den Geschlechtern unterschiedlich ausfallen können [57, 58]. GLV, z. B. (Z)-3-Hexen-1-ol, sind für einige Insekten (z. B. Melolontha hippocastani [59]) anlockend und für andere (z. B. Ostrinia furnacalis [60|) abschreckend.

Als Duftgemisch kombinierte VOC können für Insekten eine andere Bedeutung besitzen als einzeln wahrgenommene VOC. Ein und dasselbe VOC kann sowohl als WirtsbaumVOC oder als Nichtwirtsbaum-VOC fungieren, abhängig davon, in welchem Kontext, ob einzeln oder im Duftgemisch, dieses VOC wahrgenommen wird 61 63. Zum Beispiel wirken auf die Schwarze Bohnenlaus Aphis fabae 15 VOC der Ackerbohne (Vicia faba) anziehend, dagegen lösen zehn dieser VOC als Einzelsubstanz eine repellente Verhaltensreaktion bei A. fabae aus [61]. Auch das Verhältnis der einzelnen VOC zueinander, die das Duftgemisch der Wirtspflanze ausmachen, spielt eine wichtige Rolle bei der Erkennung der Wirtspflanze. Verändert sich z. B. das Verhältnis und die Konzentration der WeizenVOC zu dem des natürlichen Duftgemisches von Weizen, kann keine Attraktivität des Duftgemisches bei der Fliegenart Sitodiplosis moselana festgestellt werden 64.

Zusätzlich erlaubt das VOC-Muster von Pflanzen den Insekten nicht nur zwischen den Wirtspflanzen-Arten zu unterscheiden, sondern auch der Feststellung des Nährwertes der Wirtspflanze und die Anwesenheit von anderen Insekten auf dieser [65]. 


\subsubsection{VOC als Stressmarker}

Sich verändernde Wasserverfügbarkeit und Trockenheit sind allgemeine Stressfaktoren für viele Waldbäume und können das VOC-Muster dieser verändern [25. Bekannte Stressmarker-VOC sind GLV, die von Pflanzen als Reaktion auf Trockenheit und Herbivorenfraß emittiert werden 66 69. Nicht nur die Trockenheit an sich, sondern auch die Stärke sowie die Dauer der Trockenheit kann sich auf die VOC-Emission auswirken. Mit zunehmendem Trockenstress kommt es zu einer Zunahme der VOC-Emission von GLV 66, 69. Bei Kiefer und Eiche führt eine kurzfristige Wasserknappheit zu einer Zunahme der Monoterpen-Emissionen und einer Abnahme der Sesquiterpen-Emissionen, hält die Wasserknappheit weiter an, führt dies bei beiden Baumarten schließlich zu einer Abnahme aller Terpen-Emissionen [28, 29].

\subsection{Rot-Buche (Fagus sylvatica)}

\subsubsection{Vorkommen und Wuchsbedingungen}

F. sylvatica ist eine Schattenbaumart und die am häufigsten vorkommende dominante Laubbaumart in der potentiellen natürlichen Vegetation Zentraleuropas [6]. In der Forstwirtschaft und in der Holzindustrie zählt F. sylvatica zu den wirtschaftlich bedeutendsten Laubhölzern Europas [70.

Zur Zeit nehmen Buchenwälder von den $75 \%$ der Waldfläche, die sie in Deutschland nach der potentiellen natürlichen Vegetation einnehmen müssten, nur $21 \%$ der Gesamtwaldfläche ein [71]. Der Waldumbau zu natürlichen Waldgesellschaften ist ein wichtiges Ziel der Forstwirtschaft. Daher hat sich der Anbau von F. sylvatica auf Waldstandorten in den Jahren 2002 bis 2012 um 6 \% erhöht (102.000 ha) [71 und Monokulturen aus Nadelbaumarten wurden in den vergangenen Jahren in Mischbestände umgewandelt [72]. Der Umbau von Nadelbaumreinbeständen hin zu standortgerechten Laub- und Laubmischbeständen hat zum Ziel, den Wald für die erwarteten Belastungen durch den Klimawandel besser vorzubereiten 71 .

Das Verbreitungsgebiet von F. sylvatica erstreckt sich über das gesamte Zentraleuropa. Im Norden und im Osten Europas ist F. sylvatica eine Art, die in der Ebene, in hügeligen Gebieten und in niederen Bergländern vorkommt. Im Gegensatz dazu ist F. sylvatica im Süden und im Süd-Osten Europas eine Baumart der höheren Berglagen [6]. Ausgeprägte Sommertrockenheit und strenge Wintermonate limitieren das Verbreitungsgebiet von $F$. sylvatica 73 .

Das klimatische Optimum von F. sylvatica befindet sich im atlantisch beeinflussten Raum mit milden Wintern und nicht zu trockenen Sommermonaten 73. Eine niederschlagsreiche lange Vegetationsperiode mit einer Durchschnittstemperatur von $13^{\circ} \mathrm{C}$ sorgt 
für eine hohe Wuchsleistung von F. sylvatica 73 .

In Mitteleuropa kommen Buchenwälder von 460 bis über 2000 mm mittleren jährlichen Niederschlagsmengen vor [74. Gegenüber feuchten und sehr nassen Böden (Gley- und Aue-Böden) sowie armen Sandböden mit wechselnder Wasserversorgung ist F. sylvatica weniger tolerant [6] und verträgt keine Staunässe [75]. F. sylvatica besitzt ein hohes Toleranzpotential hinsichtlich der Bodeneigenschaften und Nährstoffversorgung im Boden und ist konkurrenzfähig sowohl auf alkanischen als auch auf sehr sauren Böden (pH-Bereich von 3 bis 7) 76 .

\subsubsection{Trockenheit}

Gegenüber Trockenheit ist F. sylvatica empfindlich [77,78. In ausgeprägten Trockenperioden kann vorzeitiger Blattfall und teilweise ein komplettes Absterben von älteren Bäumen beobachtet werden [9,79]. Nach einem sehr trockenem Jahr reagiert F. sylvatica mit einer zunehmenden Ausdünnung der Krone [80]. Des Weiteren zeigen Buchenwälder mit zunehmenden Trockenstress eine Reduktion der Feinwurzelmasse 79,81. Es wurde ebenfalls beobachtet, dass Trockenheit bei F. sylvatica zu einer Abnahme des Wurzel/SprossVerhältnisses von Sämlingen führt [82]. In einer moderaten Trockenperiode ist F. sylvatica in der Lage sich durch abnehmende Blattleitfähigkeit und Photosynthese an Trockenheit anzupassen [83]. Trocken-heiße Wetterbedingungen während des Sommers können zu einem starken Verlust der Nettoprimärfunktion von Buchenwäldern führen [84]. Während des extrem heißen und trockenen Sommers 2003 erreichte F. sylvatica im östlichen Frankreich nur $15 \%$ des durchschnittlichen Wertes der Stomataleitfähigkeit im Vergleich zum Vorjahr 84 .

Die gegenwärtigen Diskussionen zu den vorausgesagten Einflüssen des Klimawandels auf Buchenwälder 8, 85] befassen sich mit der Frage, ob die trockenstresssensible F. sylvatica in Zukunft konkurrenzfähig bleiben wird. Manthey et al. (2007) vermuten, dass Buchenwälder auf Standorten mit weniger als $650 \mathrm{~mm}$ Jahresniederschlag durch zunehmende Sommertrockenheit künftig geschädigt werden könnten 85. Auch Geßler et al. 2007 teilen die Vermutung, dass sich die Konkurrenzfähigkeit von F. sylvatica durch intensivere Trockenheitsereignisse und andere sich verändernde Umweltbedingungen im Rahmen des Klimawandels verringern wird. Wie genau andere Umweltbedingungen (wie z. B. Bodeneigenschaften und Wettbewerbsinteraktionen) die Reaktion von F. sylvatica auf Trockenheit beeinflussen, ist noch nicht genau bekannt [11]. In den südlichsten Verbreitungsgebieten zeigt F. sylvatica seit 1975 einen Wachstumsrückgang bei steigenden Temperaturen 86. 


\subsection{Insekten an Rot-Buche ( $F$. sylvatica)}

Die Insektenfauna an F. sylvatica ist relativ artenarm und bis zu fünfmal geringer als bei der Eiche 87]. Dies führen Petraktis et al. (2007) auf den hohen phenolischen Anteil in Blättern und Rinde von F. sylvatica zurück. Walentowski et al. (2010) beziffern die Anzahl der an F. sylvatica vorkommenden phytophagen Arthropodenarten, für die F. sylvatica die Haupt-Wirtspflanze ist, auf ca. 170-180 Arten [88.

Unter die an F. sylvatica vorkommenden Insektenarten fallen auch einige Arten, die deutliche Schäden an F. sylvatica anrichten können. Zu diesen Forstschädlingen zählen unter anderem zwei Arten aus der Ordnung Coleoptera, der Kleine Buchenborkenkäfer (Taphrorychus bicolor) und der Buchenprachtkäfer (Agrilus viridis), aus der Ordnung Hemiptera die Buchenblattlaus (Phyllaphis fagi) und aus der Ordnung Lepidoptera der Buchenstreckfuß (Calliteara pudibunda). Weitere Schädlinge, die an der Seite von T. bicolor und A. viridis vorkommen, sind Ernoporus fagi und der Goldgrubenprachtkäfer (Chrysobothoris affinis) [89]. An bereits gefällten Stämmen von F. sylvatica sind der Buchennutzholzborkenkäfer (Trypodendron domesticum) und der Werftkäfer (Elateroides dermestoides) zu finden [89]. Ein weiterer Schmetterling, der in Mitteleuropa aufgrund der engen Bindung an verschiedene Buchenwaldgesellschaften zu den Leitarten des Fagions zählt, ist der Nagelfleck (Aglia tau) [90].

T. bicolor befällt stehende Stämme nur, wenn sie geschwächt sind, ansonsten brütet er in absterbenden Ästen und gefällten Stämmen [89. Bei A. viridis kann es nach Dürreund Hitzejahren zu Massenvermehrungen kommen, wenn die Buchenbestände geschwächt sind [89]. Junge Pflanzen von F. sylvatica werden häufig stark von P. fagi besiedelt 91,92. Die Blattläuse sitzen meisten an der Blattunterseite von jungen Pflanzen von F. sylvatica und ernähren sich vom Phloemsaft 91,93. Massenvermehrungen von P. fagi können zu wirtschaftlichen Schäden bei Jungpflanzen und zum Absterben von geschwächten Pflanzen führen 92 . C. pudibunda ist, im Gegensatz zum A. tau, der jedoch auch bei ausreichenden Nahrungsbedingungen keine Gradationen ausbildet [90], ein Schädling an F. sylvatica 94, bei dem es immer wieder zu Massenvermehrungen in Europa kommt 95, 96.

Es wird vermutet, dass einige wärmeliebende Insekten von wärmeren und trockeneren Sommermonaten durch den Klimawandel profitieren [5, 12, mehr Generationen ausbilden [5, 12 und daher verstärkt mit Gradationen von Schadinsekten [8, 97, 98] wie dem Buchdrucker (Ips typographus) [5,12,13, zu rechnen ist. Auch der wärmeliebende C. pudibunda kann ein Profiteur des Klimawandels werden [5]. Besonders am Rande ihres potentiellen Verbreitungsgebietes kann die Anfälligkeit der Bäume durch Klimaveränderungen gegenüber Schädlingen zunehmen [98]. Zusätzlich können warme Wetterbedingungen die Flugzeiten von Schadinsekten temporär vorverlagern [99, 100].

Eine solche mögliche Entwicklung lässt sich aus den Massenausbrüchen von T. bico- 
lor, T. domesticum und A. viridis an F. sylvatica im extremen Trockensommer 2003 in Baden-Württemberg ableiten [101]. Alle drei Insektenarten sind an F. sylvatica zu Massenvermehrungen fähig und können dadurch erhebliche Schäden an den befallenen Bäumen nach einer Trockenperiode anrichten [101]. In Rotbuchenwäldern kann Insektenfraß somit in der Zukunft eine wachsende Bedeutung im Schadgeschehen zuteil werden [101].

Generell kann davon ausgegangen werden, dass durch den Klimawandel die Veränderungen im Verhalten der Borkenkäfer zunehmen, sich die Frequenz und Intensität der Schädlingsausbrüche im Waldökosystem verändern werden 12, 102 und Schadorganismen, vor allem Borkenkäferarten, Gewinner von trocken-gestressten Wirtsbäumen sein können 103 .

\subsection{Untersuchungsgebiete}

Die untersuchten Bestände von F. sylvatica liegen entlang eines 130 km langen nordwestsüdöstlichen Niederschlagsgradienten im norddeutschen Flachland in den Bundesländern Niedersachsen und Sachsen-Anhalt in den Regionen Lüneburger Heide, Wendland und Altmark im Zentrum des Verbreitungsgebietes von F. sylvatica (zw. 52 und $53^{\circ} \mathrm{N}$ und 9 und $11^{\circ} \mathrm{E}$; Abb. 1). Die Untersuchungsgebiete der Altbestände (beginnend mit den höchsten jährlichen Niederschlagsmengen) sind Sellhorn, Unterlüß, Göhrde, Klötze und Calvörde. Der klimatische Gradient zeigt einen Übergang vom mehr subozeanischen im Nordwesten bis hin zum mehr subkontinentalen Klima im Südosten mit einer kontinuierlich abnehmenden durchschnittlichen jährlichen Niederschlagsmenge von 816 bis 544 mm, einer abnehmenden durchschnittlichen Niederschlagsmenge in der Vegetationsperiode (April bis September) von 405 auf $294 \mathrm{~mm}$ und einem zunehmendem langjährigen Temperaturmittel von $8,5{ }^{\circ} \mathrm{C}$ auf $9,2{ }^{\circ} \mathrm{C}$ vom nordwestlich gelegenen Sellhorn bis zum südöstlich liegenden Calvörde (Deutscher Wetterdienst, Offenbach, Deutschland) [2].

Für die Vergleichbarkeit in Hinblick auf die bodenchemischen Bedingungen wurden Untersuchungsgebiete mit ähnlichem geologischen Substrat ausgewählt: Pleistozäne fluvioglaziale Sandablagerungen aus der letzten (Saale-)Eiszeit. Die Bestände wachsen auf sauren, nährstoffarmen sandigen oder sandig bis lehmigen Sandböden bei einer Höhenlage von 70-130 m ü. NN [2]. Die Mineralböden sind Arenosole oder Podsole (world reference base for soil recources; WRB Klassifikation) und besitzen eine 4-9 cm mächtige organische Auflage. Bodenchemische Parameter, welche die Nährstoffverfügbarkeit und den Bodensäuregehalt wiedergeben, sind in Tab. 1 aufgeführt.

Alle Standorte sind reine Altbestände von F. sylvatica oder durch F. sylvatica dominiert mit Beimischung von Traubeneiche (Quercus petraea: Sellhorn Sand, Unterlüß Sand, Klötze Sand, Calvörde Lehm: 13-19 \%) 104. Sie hatten ein Bestandesalter zwischen 115 
und 142 Jahren und besaßen ein geschlossenes Kronendach und keine erkennbare Strauchschicht. Die Bestände wurden durch Naturverjüngung begründet und die letzte Bewirtschaftung lag mehr als zehn Jahre zurück 105.

Die Messungen des Stamm-VOC-Musters wurden je Untersuchungsgebiet (Unterlüß, Göhrde und Calvörde) auf jeweils einer Versuchsfläche mit sandigem und einer mit lehmreichem Boden durchgeführt. Die Messungen des Wurzel-VOC-Musters fanden nur auf sandigen Versuchsflächen der Untersuchungsgebiete Sellhorn, Unterlüß und Klötze statt.

Die Versuchsflächen auf Sandstandorten waren durch einen höheren Anteil an grobkörnigen Bodenpartikeln und sandigen Moränenablagerungen mit wenig Schluff- und Lehmanteil und geringerer Wasserspeicherkapazität (nachfolgend als sandig bezeichnet, Sand) gekennzeichnet [2]. Dagegen wuchsen die ausgewählten Buchenbestände auf den Lehmstandorten auf lehmreichen Sandablagerungen (meist lehmhaltige oder sand-lehmhaltige Moränenablagerungen) mit höherem Schluff- und Lehmanteil und in den meisten Fällen auch höherer nutzbarer Wasserspeicherkapazität (nWSK) (nachfolgend als lehmreich bezeichnet, Lehm). Alle Informationen zu den Untersuchungsgebieten und den dazugehörigen Versuchsflächen (Sand, Lehm) können der Tab. 1 entnommen werden.

Die Versuchsflächen waren mit einem 1,5 m hohen Wildschutzzaun eingezäunt und besaßen die Größe 30 x $30 \mathrm{~m}$. 


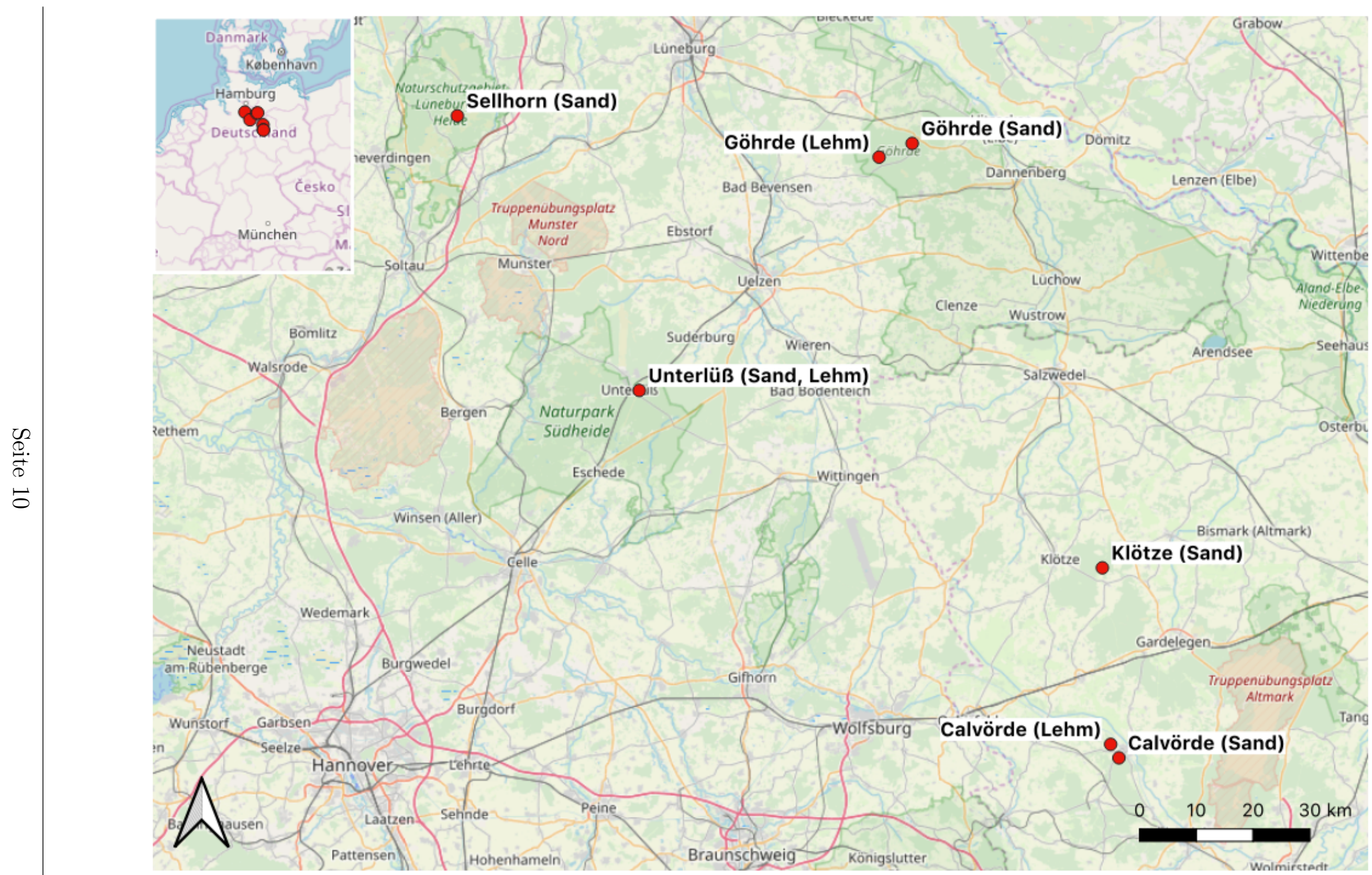

Abbildung 1: Lage der Untersuchungsgebiete und Versuchsflächen (C) 2018 Google, erstellt mit QGis 3.6.2 „Noosa“ for Mac OS X). 
Tabelle 1: Überblick der topographischen, klimatischen, edaphischen und bestandesstrukturellen Eigenschaften der untersuchten Buchenbestände entlang eines Niederschlagsgradienten im norddeutschen Tiefland. (Quelle: Müller-Haubold et al. 2013 [2], verändert)

\begin{tabular}{|c|c|c|c|c|c|c|c|c|}
\hline Untersuchungsgebiet & Sellhorn & \multicolumn{2}{|c|}{ Unterlüß } & \multicolumn{2}{|c|}{ Göhrde } & Klötze & \multicolumn{2}{|c|}{ Calvörde } \\
\hline Bodentyp & Sand & Lehm & Sand & Lehm & Sand & Sand & Lehm & Sand \\
\hline Georg. Breite & $53^{\circ} 10^{\prime} \mathrm{N}$ & $52^{\circ} 50^{\prime} \mathrm{N}$ & $52^{\circ} 50^{\prime} \mathrm{N}$ & $53^{\circ} 07^{\prime} \mathrm{N}$ & $53^{\circ} 08^{\prime} \mathrm{N}$ & $52^{\circ} 37^{\prime} \mathrm{N}$ & $52^{\circ} 24^{\prime} \mathrm{N}$ & $52^{\circ} 23^{\prime} \mathrm{N}$ \\
\hline Georg. Länge & $9^{\circ} 57^{\prime} \mathrm{E}$ & $10^{\circ} 19^{\prime} \mathrm{E}$ & $10^{\circ} 19^{\prime} \mathrm{E}$ & $10^{\circ} 49^{\prime} \mathrm{E}$ & $10^{\circ} 52^{\prime} \mathrm{E}$ & $11^{\circ} 15^{\prime} \mathrm{E}$ & $11^{\circ} 16^{\prime} \mathrm{E}$ & $11^{\circ} 17^{\prime} \mathrm{E}$ \\
\hline Höhenlage $\mathrm{m}$ ü. NHN & 130 & 120 & 117 & 85 & 85 & 85 & 72 & 75 \\
\hline Langj. Temperaturmittel $\left({ }^{\circ} \mathrm{C}\right)^{1}$ & 8,4 & 8,4 & 8,4 & 8,6 & 8,7 & 8,7 & 9,0 & 9,1 \\
\hline Temperaturmittel $2011\left({ }^{\circ} \mathrm{C}\right)$ & 9,4 & 9,6 & 9,6 & 9,6 & 9,7 & 9,8 & 10,0 & 10,1 \\
\hline Langj. Niederschlagssumme $(\mathrm{mm})^{1}$ & $816 / 405$ & $766 / 374$ & $766 / 374$ & $675 / 349$ & $665 / 347$ & $614 / 327$ & $543 / 294$ & $544 / 294$ \\
\hline Jährl. Niederschlagssumme 2011 (mm) & 810 & 710 & 710 & 677 & 666 & 573 & 509 & 515 \\
\hline Bestandesalter (a) & 127 & 115 & 115 & 142 & 133 & c. 125 & 131 & 97 \\
\hline Mittlere Baumhöhe $(\mathrm{m})^{2}$ & 28,0 & 28,4 & 25,3 & 30,2 & 24,6 & 30,2 & 28,3 & 23,8 \\
\hline Mittlerer BHD $(\mathrm{cm})^{2}$ & 29,6 & 26,1 & 18,6 & 51,0 & 30,7 & 29,0 & 36,6 & 23,4 \\
\hline Stammdichte $\left(\mathrm{N} \mathrm{ha}^{-1}\right)^{3}$ & 367 & 411 & 611 & 122 & 289 & 478 & 300 & 711 \\
\hline Bestandesgrundfläche $G\left(\mathrm{~m}^{2} \mathrm{ha}^{-1}\right)^{3}$ & 30,7 & 28,5 & 24,3 & 26,6 & 24,4 & 40,8 & 33,3 & 33,2 \\
\hline Anteil der Buche an $G(\%)$ & 95 & 100 & 81 & 100 & 94 & 81 & 97 & 100 \\
\hline Holzvorat $\left(\mathrm{m}^{3} \mathrm{ha}^{-1}\right)^{2}$ & 395 & 378 & 227 & 407 & 289 & 516 & 454 & 374 \\
\hline Dicke der organ. Auflage $(\mathrm{cm})$ & 9,1 & 6,5 & 7,3 & 9,0 & 8,8 & 6,4 & 7,5 & 6,6 \\
\hline pH-Wert $\left(\mathrm{H}_{2} \mathrm{O}\right)^{4}$ & 4,2 & 4,4 & 4,3 & 4,3 & 4,3 & 4,3 & 4,2 & 4,3 \\
\hline $\mathrm{C} / \mathrm{N}-$ Verhältnis $\left(\mathrm{g} \mathrm{g}^{-1}\right)^{4}$ & 24,2 & 25,8 & 24,1 & 22,9 & 25,5 & 15,7 & 15,9 & 13,2 \\
\hline N Konzentration $\left(\mathrm{g} \mathrm{kg}^{-1}\right)^{4}$ & 0,46 & 0,40 & 0,47 & 0,41 & 0,51 & 0,49 & 0,34 & 0,42 \\
\hline P Konzentration $\left(\mathrm{mg} \mathrm{kg}^{-1}\right)^{4}$ & 79 & 71 & 106 & 59 & 97 & 97 & 126 & 111 \\
\hline $\mathrm{Al}$ Konzentration $\left(\mathrm{g} \mathrm{kg}^{-1}\right)^{4}$ & 0,52 & 0,58 & 0,31 & 0,46 & 0,79 & 0,54 & 0,38 & 0,57 \\
\hline $\operatorname{AKe}\left(\mu_{m o l} g^{-1}\right)^{4}$ & 27,5 & 18,4 & 24,2 & 20,2 & 26,5 & 17,3 & 18,6 & 14,7 \\
\hline Basensättigung $(\%)^{4}$ & 9,7 & 14,8 & 8,3 & 6,7 & 2,8 & 9,7 & 7,4 & 5,0 \\
\hline Anteil feinkörniger $(<200 \mu \mathrm{m})$ Partikel $(\%)^{5}$ & 42 & 52 & 46 & 41 & 14 & 46 & 61 & 24 \\
\hline $\mathrm{nWSK}(\mathrm{mm} / 120 \mathrm{~cm})^{6}$ & 111 & 95 & 79 & 78 & 107 & 90 & 140 & 81 \\
\hline
\end{tabular}

$1=$ langjähriges Mittel von 1971-2000 (regionalisiert von C. Döring aus der Wetterstation-Datenbank, die vom Deutschen Wetterdienst zur Verfügung gestellt wurde); Jahresniederschlag/Niederschlag April-September

${ }^{2}=$ Brusthöhendurchmesser (BHD) und Holzvorrat beziehen sich auf alle Buchen $>7 \mathrm{~cm}$ Stammdurchmesser, Baumhöhe bezieht sich auf alle Buchen mit Anteil an der oberen Kronenschicht

$3=$ Stammmdichte und Bestandesgrundfläche G schießen alle Baumarten mit einem Durchmesser $>7 \mathrm{~cm}$ je Versuchsfläche ein

$4=$ bodenchemische Eigenschaften aus einer zentralen Profilgrube bis $30 \mathrm{~cm}$ Bodentiefe (N: Gesamtstickstoff; $\mathrm{Al}$ : $\mathrm{NH}_{4} \mathrm{Cl}$ austauschbares $\mathrm{Al}$; P: Gesamtphosphor; Daten wurden von M. Jansen und C. Döring zur Verfügung gestellt)

${ }^{5}=$ feinkörnige Bodenpartikel schließen feinen Sand, Schluff und Lehm (Partikelgröße $<200 \mu \mathrm{m}$ ) mit ein und werden als \% der gesamten Bodenmasse (0-120 cm Bodentiefe) angegeben

$6=$ nutzbare Wasserspeicherkapazität nWSK als Mittel der oberen $120 \mathrm{~cm}$ Bodentiefe 


\subsection{Methodik der VOC-Analyse}

\subsubsection{Gaschromatographie}

Die Chromatographie ist ein physikalisch-chemisches Trennverfahren und nutzt die Verteilung des einzelnen Stoffes zwischen zwei miteinander nicht mischbaren Phasen, einer stationären und einer mobilen Phase, um flüchtige Stoffgemische aufzutrennen [106, 107. Zwischen beiden Phasen findet ein kontinuierlicher Stoffaustausch statt [108. Für die Auftrennung eines Stoffgemisches spielen zwei Vorgänge, Adsorption und Desorption, welche entgegengerichtet sind und somit miteinander in Konkurrenz stehen, eine wichtige Rolle [109. Der Sorptionsprozess wird von der stationären Phase verursacht, an dem die Substanzen durch Adsorption an dem Trägermaterial festgehalten werden. Beim Desorptionsprozess, welcher von der mobilen Phase verursacht wird, werden die Substanzen vom Träger abgelöst und weitertransportiert 109.

In der Gaschromatographie wird dieses Trennverfahren von Stoffgemischen verwendet und erlaubt so die Auftrennung von sehr komplexen Stoffgemischen. Charakteristisch bei der Gaschromatographie ist, dass die mobile Phase, welche als Transportmittel für das Stoffgemisch dient, gasförmig ist. Die stationäre Phase, welche die Probebestandteile festhält, kann entweder flüssig oder fest sein [107]. Als Trägergas werden Helium, Stickstoff oder Wasserstoff verwendet. Von der Reinheit des verwendeten Gases hängt die Nachweisgrenze des Stoffes ab. Die zu trennenden Stoffe müssen in die Gasphase überführbar sein. Das bedeutet, die chemischen Verbindungen müssen einen Siedepunkt von max. $300^{\circ} \mathrm{C}$ besitzen und ausreichend thermostabil sein [107. Die zu analysierenden Stoffgemische (Proben) sind bei Raumtemperatur meist flüssig und liegen in der Regel in einem Lösungsmittel verdünnt vor 107.

Die Verweildauer der Substanzmoleküle in der Trennsäule hängt davon ab, wie lange diese zwischen der stationären und mobilen Phase hin und her wechseln [110]. Die Polarität der stationären Phase hat einen Einfluss auf die Verweildauer. So treten z. B. polare Verbindungen mit polaren stationären Phasen in Wechselwirkung und werden entsprechend ihrer Polarität zurückgehalten [111]. Die Retentionszeit ( davon ab, wie lange sich die jeweilige Substanz in der stationären Phase befindet, und ist die Zeit, die eine Substanz benötigt, um nach der Probeaufgabe die Säule zu passieren und am Detektor registriert zu werden. Die RT entspricht somit der Zeitdifferenz zwischen Injektion und Detektion [109. 


\subsubsection{Massenspektrometrie}

In dieser Arbeit wurde ein Gaschromatograph (GC) gekoppelt mit einem Massenspektrometer (MS) verwendet. Im Massenspektrometer werden die einzelnen Verbindungen ionisiert, indem sie mit Elektronen beschossen werden. Dabei zerfällt ein großer Teil der Moleküle in charakteristische Bruchstücke (Fragmentierung). Die Masse der Bruchstücke wird mit dem MS erfasst und liefert Informationen zur Struktur der Ausgangsverbindung. Durch Abgleich des Fragmentierungsmusters in den Massenspektren aus Datenbanken kann die jeweilige Substanz identifiziert werden [111.

Die elektrischen Signale, die das MS erzeugt, werden als Massenspektren ausgegeben. Auf der y-Achse wird die Intensität und auf der x-Achse das Masse-Ladungs-Verhältnis der verschiedenen Molekülbruchstücke der zuvor chromatographisch getrennten Verbindung wiedergegeben 106. Die in einem Massenspektrum vorkommenden Peaks können in Molekülionen-Peaks, Fragmentations-Peaks und Basis-Peaks unterschieden werden. Der Molekülionen-Peak ist der Peak, bei dem das Masse/Ladungs-Verhältnis $\mathrm{m} / \mathrm{z}$ ) der Molmasse der Verbindung entspricht. Peaks mit bekannten Fragmentationsbruchstücken, die bei der jeweiligen Verbindung bei der Ionisation entstehen, werden Fragmentations-Peaks genannt. Der Peak, der mit der höchsten Intensität vorkommt, wird als Basis-Peak bezeichnet und zur Berechnung der relativen Intensität verwendet (Abb. 2) [106].

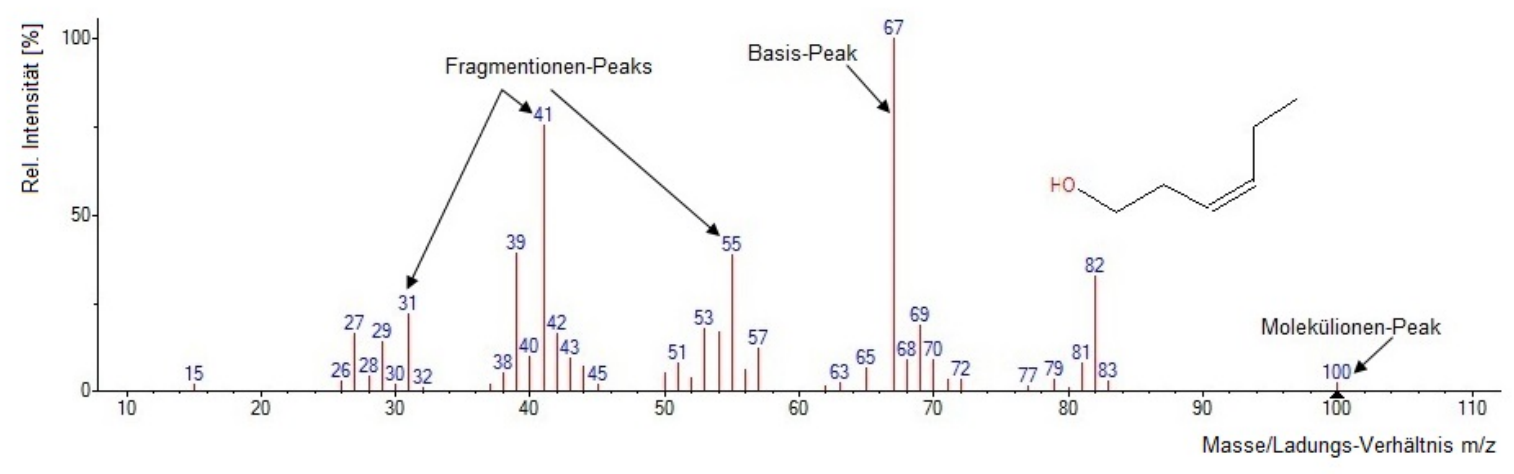

Abbildung 2: Massenspektrum von (Z)-3-Hexen-1-ol (verändert nach der NISTDatenbank (MS Search 2.0)) 


\title{
Stamm-Volatilenmuster von Rot-Buche (F. sylvatica)
}

\author{
Christine Rachow
}

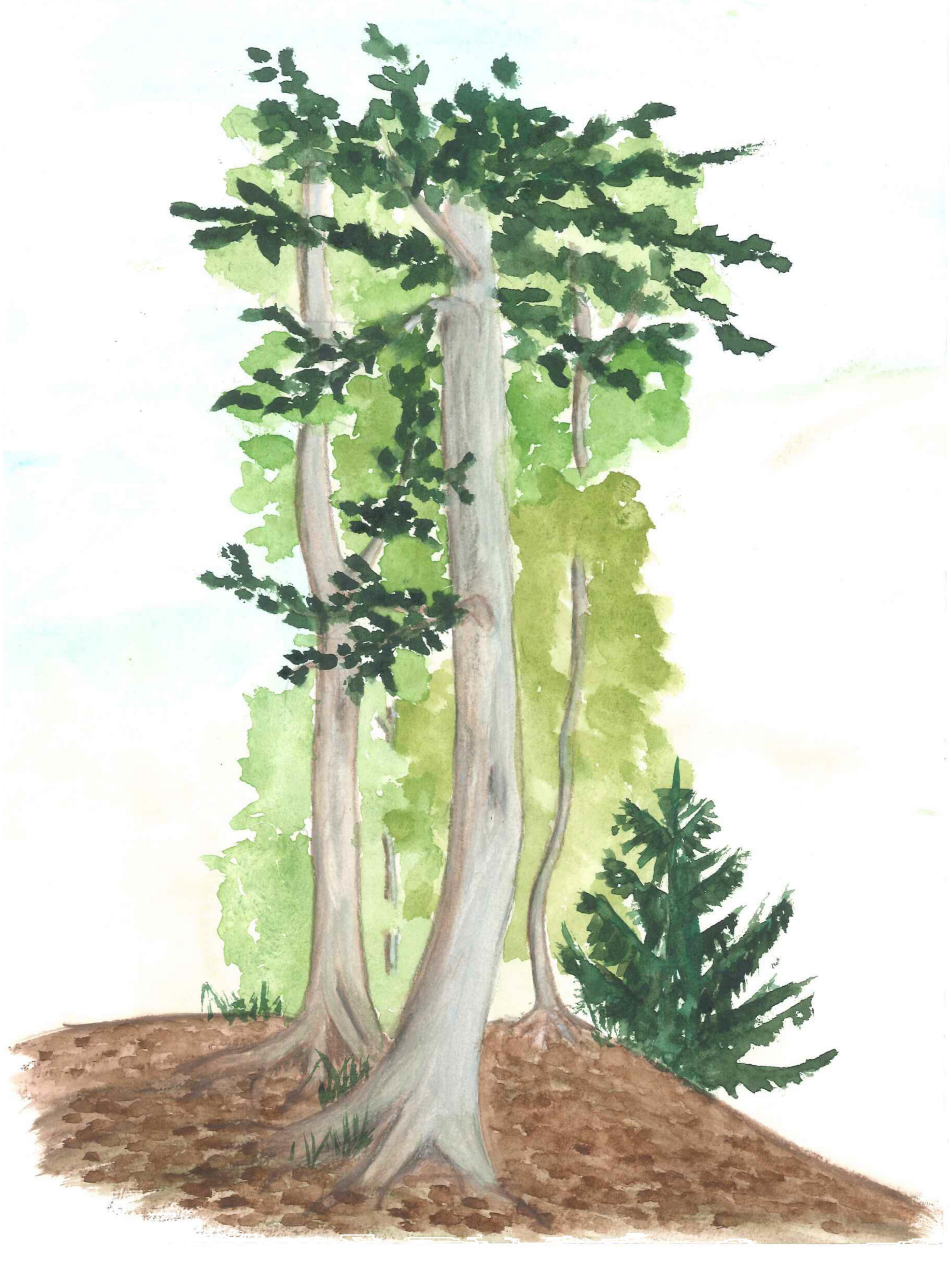

Abbildung 3: Darstellung eines Stammes von F. sylvatica

Teile des Inhaltes dieses Kapitels wurden in „Mitteilungen der Deutschen Gesellschaft für allgemeine und angewandte Entomologie“" veröffentlicht, Band 18 (2012): 635-639, ISSN: 0344-9084 


\section{Stamm-Volatilenmuster von Rot-Buche ( $F$. sylvatica)}

VOC spielen eine wichtige Rolle als Infochemikalien und tragen zu Interaktionen von Lebewesen über mehrere trophische Ebenen bei $[112$. Die Abgabe der VOC erfolgt über alle Bestandteile der Pflanze [112], wobei ihre Funktion als Infochemikalien vielfältig sein kann und unter anderem der Abwehr gegen Herbivore dient [113, 114 sowie Aufschluss über den Fitnesszustand der Pflanze selber geben kann [115]. Ist ein Baum geschwächt, emittiert dieser VOC, die den umstehenden Bäumen und Insekten als Signalstoffe von Nutzen sein können [116.

Insekten nutzen VOC auch, um eine geeignete Wirtspflanze für die Eiablage zu finden 18, 117, 118. Abiotische und biotische Umweltbedingungen können dabei die Produktion und die Freisetzung verhaltenswirksamer VOC durch die Pflanze beeinflussen und sich auf die Eiablage-Präferenzen auswirken [18]. Einige Insektenarten wählen bei ihrer Suche nach einem geeigneten Eiablageplatz geschwächte Bäume im Bestand aus [119. So nutzen Borkenkäferarten Ethanol, einen Alkohol, welcher von gestressten Pflanzen freigesetzt wird, um geeignetes Brutmaterial zu finden [120]. An F. sylvatica vorkommende Lepidoptera-Arten können buchen-spezifische Rinden-VOC wahrnehmen [121] (s. Kapitel 5 und anhand dieser möglicherweise geeignetes Brutmaterial ausfindig machen.

In diesem Kapitel wird nach Marker-VOC gesucht, die zur Früherkennung eines Insektenbefalls in einem durch Trockenheit gefährdeten Buchenwald dienen können. Um Stämme von stehenden Altbuchen auf ihr VOC-Muster beproben zu können wurde eine Stammabsaugkammer entwickelt (s. Kapitel 3.2), die die Beprobung im Vergleich zu bisherigen Methoden vereinfacht. Die Entwicklung wurde in einem Vorversuch auf ihre Einsatzmöglichkeiten (s. Kapitel 3.3) untersucht. Anschließend wurde mit der entwickelten Methode das Stamm-VOC-Muster von F. sylvatica in den Untersuchungsgebieten Unterlüß, Göhrde und Calvörde untersucht (s. Kapitel 3.4). Alle drei Untersuchungsgebiete liegen in dem Hauptverbreitungsgebiet von F. sylvatica in der Lüneburger Heide und weisen unterschiedliche jährliche Niederschlagsmengen auf.

Nach einem theoretischen Hintergrund (s. Kapitel 3.1) werden in diesem Kapitel folgende Forschungsfragen mit zugehörigen Hypothesen untersucht:

Forschungsfrage 1: Ändert sich im jahreszeitlichen Verlauf die Stoffklassen-Zusammensetzung des Stamm-VOC-Muster von F. sylvatica?

Hypothese 1: Es gibt Veränderungen der Stoffklassenzusammensetzung im StammVOC-Muster von F. sylvatica innerhalb einer Vegetationsperiode.

Forschungsfrage 2: Unterscheidet sich das Stamm-VOC-Muster von F. sylvatica auf drei verschiedenen Standorten mit unterschiedlichen jährlichen Niederschlagsmengen? 
Hypothese 2: Das Stamm-VOC-Muster von F. sylvatica von drei verschiedenen Standorten unterscheidet sich in der chemischen Stoffgruppenzusammensetzung.

Forschungsfrage 3: Gibt es spezifische Stamm-VOC von F. sylvatica, die als mögliche Stressmarker für durch Trockenstress geschwächte Bestände verwendet werden können?

Hypothese 3: Es gibt mindestens ein VOC, dass als Marker-VOC zur Früherkennung von trocken-gestressten Stämmen von F. sylvatica genutzt werden kann. 


\subsection{Theoretischer Hintergrund}

\subsubsection{Rot-Buchenstamm}

F. sylvatica ist eine geradwüchsige Baumart mit einem vorwiegend astfreien Stamm. Freistehende Rot-Buchen sind aufgrund der dünnen Rinde empfindlich gegenüber Sonnenbrand und bleiben im Freistand bis zum Boden beastet. Ansonsten bildet F. sylvatica im Bestand einen bis zu $45 \mathrm{~m}$ hohen Stamm aus [70].

Im Alter kann es zur Bildung eines rotbraunen Falschkerns, dem Rotkern, im Holz kommen. Die Ausdehnung dieses Rotkerns nimmt meist im Stammquerschnitt von unten nach oben zu. Der Anteil der verkernten Stämme steigt mit Bestandesalter und Stammdurchmesser und kann bis zu 90 \% der Bäume in einem 170 Jahre alten Bestand ausmachen [122. Bekannte farbkerntypische VOC sind Phenylethanol, 1-Octanol und Nonanal 123 .

Der Stamm von F. sylvatica ist unterschiedlichen Einflüssen ausgesetzt, die sich auf das Stamm-VOC-Muster auswirken können. Dazu zählen die Umwelt- und Bodenbedingungen, die z. B. das Dickenwachstum des Stammes beeinflussen, aber auch Buchenerkrankungen. Zu diesen gehören Pilzarten wie z. B. Nectria ditissima, welche Baumkrebs verursachen, und Holzfäuleerreger (z. B. Fomes fomentarius) [70|. Bei der Entstehung von Infektionspfaden spielt die Buchenwollschildlaus (Cryptococcus fagisuga) eine Rolle, indem sie kleinste Verletzungen in der Rinde verursacht, die als Eintrittspforten für Sekundärpathogene dienen. Ein solcher Sekundärpathogen ist der Pilz Nectria coccinea, der für die Buchenschleimflusskrankheit verantwortlich ist [70]. In dem Bereich der Schleimflecken sind häufig holzbohrende Insekten (Gestreifte Nutzholzborkenkäfer (Trypodendron lineatum), Werftkäfer (E. dermestoides)) anzutreffen, dessen Fraßgänge das Eindringen weiterer Pathogene (vor allem Weißfäuleereger) ermöglichen [70]. Die als Buchenkomplexkrankheit oder auch Buchenrindennekrose bekannte Erkrankung ist ein Aufeinanderfolgen mehrerer Schadereignisse, an dem neben $N$. coccinea auch die C. fagisuga maßgeblich beteiligt ist 124,125 .

\subsubsection{Oberirdische VOC-Muster von F. sylvatica}

Während oberirdisch das VOC-Muster der Zweige und Blätter von F. sylvatica gut erforscht ist 39, 126 128 (Tab. A.1), gibt es bisher nur wenig über das Stamm-VOCMuster von F. sylvatica. Entsprechend ersten Versuchen verändert sich das Stamm-VOCMuster von F. sylvatica vom Frühjahr bis zum Herbst. Andere VOC wie Hexanal, $\alpha$-Pinen und Camphen werden über das ganze Jahr von den Stämmen von F. sylvatica emittiert [129] (s. Kapitel 3.2).

Zu dem VOC-Muster der Zweige und Blätter zählen die typischen Grünblattdüfte (GLV, 
green leaf volatiles) wie (Z)-3-Hexen-1-ol und (Z)-3-Hexenylacetat, die von den Blättern emittiert werden [127, 128, 130], und Monoterpene (MT) ( $\alpha$-Pinen, $\beta$-Pinen, 3-Caren, Limonen und Sabinen), die von den belaubten Zweigen abgegeben werden [39, 40, 126. Sabinen gehört dabei sowohl im VOC-Muster der Blätter als auch der Zweige zu einem der dominantesten VOC [39,40,127, 130].

Allgemein ist das VOC-Muster von F. sylvatica oberirdisch von Terpenen geprägt 40, 126]. MT-Emissionen von Zweigen von F. sylvatica weisen einen ausgeprägten temperaturabhängigen Tagesgang mit entsprechenden „Nachmittagsoptimum“ bei maximalen Temperaturen am Tag auf [131 133]. Zusätzlich zeigen MT von Buchenzweigen jährliche Schwankungen in den Emissionsraten [126]. Hohe Emissionsraten wurden von Holzke et al. (2006) im Sommer bei hohen Temperaturen und hoher photosynthetisch wirksamer Strahlung und geringe Emissionsraten im Herbst gemessen [126].

Zusätzlich besitzen auch Stressereignisse einen Einfluss auf das oberirdische Blatt-VOCMuster von F. sylvatica. Die Untersuchung von Šimpraga et al. 2011 konnte eine Verbindung zwischen der MT-Abgabe bei jungen Pflanzen von F. sylvatica und dem Grad der Trockenheit nachweisen 25. Demnach wurde die Abgabe von MT durch Trockenstress beeinflusst. MT-Emissionen nahmen am Anfang des Stresses zu, um dann bei andauerndem Trockenstress abzunehmen. Auch bei anschließender Wässerung nahmen die MTEmissionen weiterhin ab, um dann ihren tiefsten Wert zu erreichen. 


\title{
3.2. Ein neues Verfahren zum Sammeln von Stammvolatilen ${ }^{1}$
}

\author{
Christine Rachow, Patrik Gester-Gerstmann, Björn Weis \& Stefan Schütz \\ Department of Forest Zoology and Forest Conservation, Büsgen-Institute, \\ Georg-August-University, Göttingen, Germany
}

\begin{abstract}
A lightweight stem enclosure for sampling of trunk volatiles
Volatiles (Volatile Organic Compounds, VOC) play an important role as cues for host plant finding and host plant choice. In contrast to leaf volatiles, the release of stem volatiles was only seldom subject of examination. However, stem volatiles are considered to be of significant importance during the host finding process of xylophagous insects.

This study presents a new method for sampling of stem volatiles with reduced weight and time expenditure. The method is based on an oven bag and an extraction equipment. The oven bag is used to constitute an enclosure around a section of a stem. The air in the enclosure is constantly extracted for two hours. The samples are individually trapped on Tenax and desorbed thermally on a TDS (ThermalDesorptionSystem) system for final sample analysis on a gas chromatograph with a mass selective detector (GC-MS).

The advantages of the method are the reduced time expenditure for the installation, the low costs for the flexible tubes and their low weight in contrast to the common enclosures made from steel and PTFE.
\end{abstract}

Key words: Fagus sylvatica, drought stress, stem volatiles, air tightness, volatile organic compounds

Christine Rachow, Büsgenweg 3, 37077 Göttingen, Germany, E-Mail: crachow@gwdg.de

\subsubsection{Einleitung}

Es ist bekannt, dass Bäume Duftstoffe über ihre Blätter freisetzen. Die Zusammensetzung der Duftstoffe variiert von Pflanze zu Pflanze und hängt vom Entwicklungsstadium der Pflanze sowie dem jeweiligen Individuum selbst ab [134-136]. Als luftübertragbare Signale haben sie eine wichtige Funktion bei der Kommunikation zwischen Pflanzen, aber auch zwischen Pflanzen und Insekten [113, 137, 138.

Sowohl über Blätter als auch über den Stamm geben Bäume Duftstoffe ab. Der Stamm als Duftstoffquelle ist im Gegensatz zu den Blättern kaum erforscht.

Für eine Abschätzung der ökologischen und physiologischen Relevanz der Stamm-Volatile

${ }^{1}$ Dieses Kapitel wurde publiziert unter: C. Rachow (2012) / Mitt. Dtsch. Ges. Allg. Angew. Ent. 18, S. 635-639 
(VOC, Volatile Organic Compounds) ist eine quantitative und qualitative Analyse der Volatilenabgabe durch den Baumstamm wichtig.

Um die emittierten Volatile effektiv aufzufangen und diese mit Hilfe von gaschromatographischen Methoden (wie Gaschromatographie mit Massenspektrometrie-Kopplung; GC-MS) zu analysieren hat sich die Methode der Stammabsaugkammer bewährt [139.

Die grundsätzliche Anwendung der bisherigen Methoden zur Stammabsaugkammer sieht einen isolierten Raum mittels Edelstahl und Teflonfolie vor, der um einen Baumstamm geschaffen wird. In diesem Raum können sich die vom Stamm emittierten Volatile anreichern, die mit Hilfe einer Pumpe auf einem Absorptionsmittel gesammelt werden. Mit diesen Stahlkammern lassen sich Stammvolatile nachweisen, doch ist die Kammer teuer, schwer und somit unhandlich. Ziel dieser Studie ist daher eine günstige, leichtgewichtige und handhabbare Stammabsaugkammer zu beschreiben.

\subsubsection{Material und Methoden}

Das Prinzip der Absaugkammer besteht darin, dass Außenluft durch einen Filter gereinigt in eine isolierte Kammer gelangt und sich dort mit baumbürtigen Volatilen anreichert. Anschließend wird diese Luft mittels einer Drehschieberpumpe (Typ DC 12/16 Fk, Firma Fürgut, Tannheim) über ein Absorptionsmittel (Tenax ${ }^{\circledR}$ TA TDS Röhrchen, Firma Gerstel, Mühlheim, D) gesaugt.

Die Absaugkammer besteht aus einem handelsüblichen Bratschlauch (Typ Nalophan (Polyethylenterephthalat), Länge $25 \mathrm{~m}$, Höhe $46 \mathrm{~cm}$, Firma Kalle, Wiesbaden, D) in dem vorher zwei Teflonadapter (Eigenbau) eingelassen worden sind (Abb. 4 a). Der Bratschlauch wird in Brusthöhe um den zu beprobenden Stamm angebracht. Die Enden des Bratschlauches werden übereinander gelegt, mehrfach gefaltet und von außen mit Leukoplast ${ }^{\circledR}$ fixiert (Abb. 4). Je nach Stammumfang wird der Bratschlauch in die entsprechende Länge zugeschnitten. Bei einem Umfang von $90 \mathrm{~cm}$ empfiehlt sich ein Bratschlauchstück von $150 \mathrm{~cm}$ Länge (Stammumfang plus $60 \mathrm{~cm}$ ). Vor jedem einmaligen Gebrauch werden die Bratschläuche bei $60{ }^{\circ} \mathrm{C}$ für jeweils zwei Stunden ausgeheizt. Anschließend werden sie luftdicht mit Alufolie umwickelt, um mögliche Kontaminationen mit der Umgebungsluft zu vermeiden. Die Vorfixierung der Kammer erfolgt mit Zurrgurten mit Klemmverschluss im $20 \mathrm{~cm}$ Abstand (Abb. 4 a) zueinander. Dieser Abstand entspricht der Höhe des Innenraumes der Stammabsaugkammer.

Um einen zweimaligen Luftwechsel des Innenraumes bei einer 80-100-jährigen Buche zu gewährleisten, wird der Mindestabstand von $2 \mathrm{~cm}$ zwischen Bratschlauchfolie und Stammoberfläche angesetzt. Mit dem Mindestabstand zwischen Folie und Oberfläche berechnet sich das Innenvolumen der Stammabsaugkammer aus dem Produkt von Höhe, Pi und der Differenz aus dem Radiusquadrat des Außendurchmessers der Stammabsaugkammer und 
Radiusquadrat des Stammes. Mittels einer Heißluftpistole wird der Bratschlauch oberhalb der Zurrgurte an den Baum geschrumpft. Dadurch werden die Ränder des Bratschlauches an die Struktur der Rinde angepasst. Anschließend können die Zurrgurte entfernt werden. Die eigentliche Fixierung des Bratschlauches am Stamm erfolgt mit einlagigen Isomatten (Polyethylen-Schaum) und vier Spanngurten mit Ratschenverschluss. Dafür werden die Isomatten in $10 \mathrm{~cm}$ breiten Streifen geschnitten und von außen an die obere und untere Abschlusskante des Bratschlauches angelegt (Abb. 4). Die Isomatten dichten dabei das System ab, in dem sie sich beim Spannen der Spanngurte an die Unebenheiten des Baumstammes anpassen. Des Weiteren schützen sie die Oberseite des Stammes vor Quetschungen durch die Spannvorrichtung. Die zwei Spanngurte werden in gegensätzlichen Zugrichtungen an die jeweilige Abschlusskante angebracht, um die Isomatten und den darunter befindlichen Bratschlauch luftdicht an den Baum anzuziehen. Durch die verschiedenen Zugrichtungen wird die Krafteinwirkung auf den Bratschlauch unterschiedlich verteilt und erzeugt, wie Vorversuche zeigten, eine höhere Dichtigkeit als bei nur einem Spanngurt. Die Teflonadapter dienen als Verbindungsstücke zwischen dem Bratschlauch und der Drehschieberpumpe mit dem Absorptionsmittel sowie zum Luftfilter. Dafür werden an dem Adapter Teflonschläuche befestigt, die dann zu dem Absorptionsmittel und dem Luftfilter führen (Abb. 4 a). Als Luftfilter eignen sich Tenax ${ }^{\circledR}$ TA Röhrchen oder CLSA Röhrchen (closed-loop stripping analysis, Aktivkohle-Filter, Gränicher Daniel, Daumazan, F). An Fagus sylvatica hat sich eine Beprobungszeit von zwei Stunden bewährt.

Die Bratschläuche werden nur einmal verwendet. Das Reinigen der wieder verwendbaren Teflonschläuche und Teflonadapter erfolgt mit $70 \%$ Ethanol (unvergällt) und demineralisiertem Wasser. Anschließend werden die Teflonmaterialien für zwei Stunden bei $120{ }^{\circ} \mathrm{C}$ ausgeheizt und danach in Alufolie eingewickelt, um eine Anreicherung von Fremdvolatilen $\mathrm{zu}$ vermeiden.

Um die Dichtigkeit dieser Methode zu überprüfen, wurden Laborversuche durchgeführt. Hierbei wurde an $80 \mathrm{~cm}$ hohen Stammrollen (Durchmesser zw. 18-22 cm) von F. sylvatica die Absaugkammer installiert. Der gesamte Aufbau wurde von einem weiteren zweiten äußeren Bratschlauch umgeben, in den eine definierte Menge einer nicht vom Stamm kommende Testsubstanz (5-Methylfurfural 98 \% (Firma Acros); 1:1000 Verdünnung in Paraffinöl Uvasol (Firma Merck)) eingebracht wurde. Dafür wurden fünf Tropfen (ca. 150 pl) der Verdünnung 5-Methylfurfural in Paraffinöl auf jeweils ein Filterpapierstück $\left(4 \mathrm{x} 4 \mathrm{~cm}^{2}\right.$ (Firma Macherey-Nagel)) gegeben. Drei Glaspetrischalen mit jeweils einem Filterpapierstück wurden gleichmäßig verteilt in den äußeren zweiten Bratschlauch gestellt. Zusätzlich zur inneren Absaugkammer wurde auch die Luft außerhalb im äußeren Bratschlauch mittels Drehschieberpumpe und dem Absorptionsmittel Tenax ${ }^{\circledR}$ TA beprobt. Anschließende Analysen mittels GC-MS ergaben den Anteil der Testsubstanz, der 
aus dem Außenbereich in den Innenbereich der Absaugkammer gelangt ist.
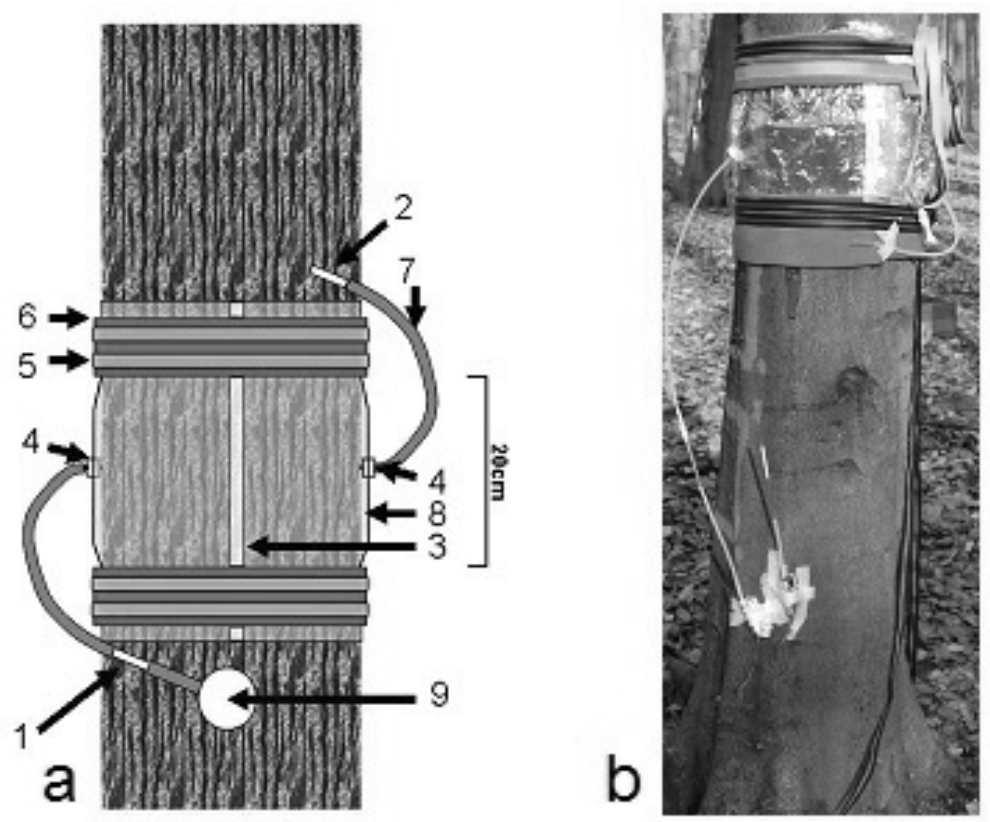

Abbildung 4: Stammabsaugkammer a) Modell und b) Foto: 1: Probe (Tenax ${ }^{\circledR}$ TA), 2: Luftfilter (Tenax ${ }^{\circledR}$ TA oder CLSA), 3: Schnittstelle des Bratschlauches mit Leukoplast ${ }^{\circledR}$ fixiert, 4: Teflonadapter, 5: Spanngurt mit Ratschenverschluss, 6: Isomatte, 7: Teflonschlauch, 8: Bratschlauch, 9: Drehschieberpumpe

\subsubsection{Ergebnisse}

Mit der Leichtbau-Stammabsaugkammer können auch bei niedrigen Temperaturen im Winter noch Stamm-Volatile gemessen werden (Abb. 䣕 a und b). Es wird aber deutlich, dass der Stamm bei wärmeren Temperaturen eine höhere Anzahl und eine größere Mengen an Stamm-Volatilen freisetzt. Exemplarisch sind zwei Messungen von je einem Buchenstamm im August und im November 2010 in Abb. 5 a und b dargestellt.

Die Überprüfung der Dichtigkeit mittels Tenax ${ }^{\circledR}$ TA Röhrchen als Luftfilter an neun Teststämmen ergaben einen Mittelwert von $97 \pm 6,5 \%$. Im Mittel sind bei den Laborversuchen nur 3,1\% der Testsubstanz aus dem äußeren zweiten Bratschlauch in die eigentliche Stammabsaugkammer gelangt. Die Peakfläche der Störsubstanz (5-Methylfurfural) ist somit im Innenraum der Stammabsaugkammer nach der ISO-Norm 16000-6:2004 unter der erforderlichen $10 \%$, die nötig sind, damit eine Duftprobe noch ausgewertet werden kann 140.

\footnotetext{
${ }^{2}$ Analyse der TDS-Proben s. S. 26
} 


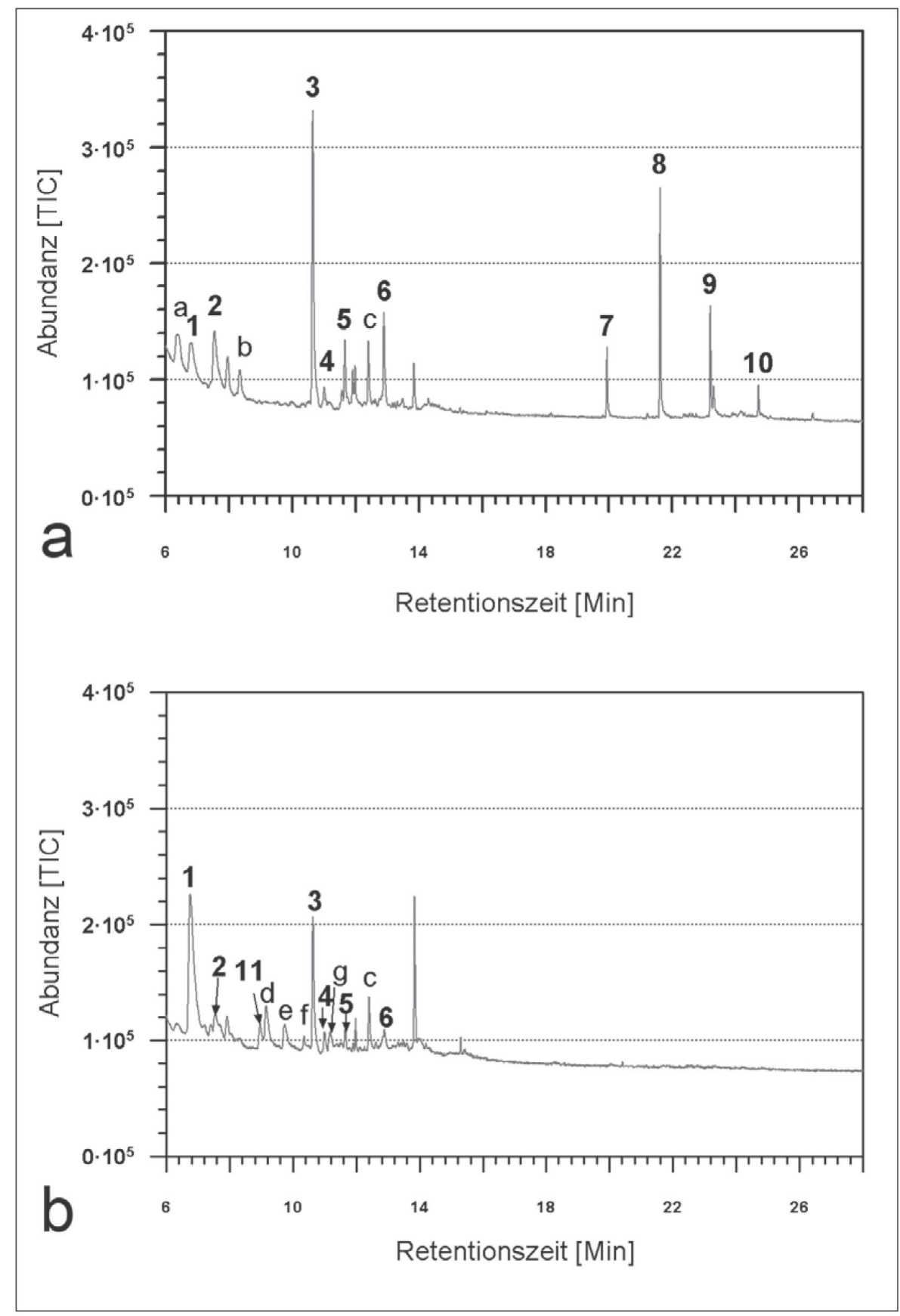

Abbildung 5: Total Ionenchromatogramm [TIC] der TDS-Proben im Jahr 2010 von $F$. sylvatica a) im August $\left(+20^{\circ} \mathrm{C}\right)$ und b) im November $\left(-1{ }^{\circ} \mathrm{C}\right)$.

Buchstaben: Identifizierung der Volatile mit dem Mass Spectral Search Library of the National Institute of Standards and Technology NIST (Gaithersburg, USA). a: 2-Methyl-1-pentanal; b: 2-Ethyl-3-methylbutanal; c: 3-Caren; d: p-Xylol; e: o-Xylol; f: Tricyclene; g: 2-Ethylhexanal

Zahlen: Zusätzliche Identifizierung mit authentischen Standards. 1: Toluol; 2: Hexanal; 3: $\alpha$-Pinen; 4: Camphen; 5: $\beta$-Pinen; 6: Eukalyptol; 7: Tetradecan; 8: Pentadecan; 9: Hexadecan; 10: Heptadecan; 11: 2-Ethylhexanal 


\subsubsection{Diskussion}

Der Aufbau der Methode wurde einfach gehalten und spiegelt sich im schnellen Aufund Abbau der Stammabsaugkammer wieder. Wichtig für den Aufbau im Gelände ist die Vorbereitung im Labor. Dadurch kann viel Zeit im Gelände gespart werden, was besonders bei schlechten Wetterbedingungen empfehlenswert ist. Bei starkem Regen kann nur gemessen werden, wenn für die Pumpen und die Absorptionsmittel ein Wetterschutz vorhanden ist. Die Einarbeitung in diese Methode ist einfach zu erlernen, da beim Aufbau eine geringe Zahl von Handgriffen im Gelände nötig ist.

Wichtig bei den verwendeten Materialien ist die geringe Abgabe und Aufnahme von Duftstoffen sowie das einfache und günstige Beschaffen der Hauptkomponente Bratschlauch. Im Vergleich zu der Stammabsaugkammern aus Stahl und PTFE 139 ist das Reinigen der Leichtbau-Kammer mit einem geringeren Zeitaufwand verbunden. Das aufwendige Reinigen der Innenseite der Kammer wird bei der Bratschlauch-Variante umgangen, da bei dieser nach jedem Versuch aufgrund des günstigen Preises die komplette Innen- und Außenseite der Stammabsaugkammer in Form des Bratschlauches entsorgt werden kann. Dadurch wird verhindert, dass sich Fremdvolatile an die Oberseite festsetzten wodurch ein Memory-Effekt vermieden wird.

Im Gegensatz zu den Stahlkammern (je nach Größe der Kammer ca. 10,5-19,5 kg) wiegt diese Kammer deutlich weniger (ca. $1 \mathrm{~kg}$ ) und ist in ihren Abmessungen handlicher. Sie kann im Gegensatz zu der Stahlvariante von nur einer Person an den Stamm angebracht werden. Beim alleinigen Aufbau werden dabei nicht mehr als 30 Minuten pro Baum benötigt (bis zu einer Stunde bei der Stahlkammer). Ein weiterer Vorteil dieser Kammer ist, dass sie sich für jeden Stammdurchmesser eignet, da der Bratschlauch und die Isomatten zugeschnitten werden können.

Diese Methode kann auch auf andere Baumarten angewendet werden. Neben der Buche wurde diese Methode auch an Fichte getestet. Kleine Zweige oder Aststümpfe sind beim Anlegen des Bratschlauches keine Schwierigkeit. Dagegen kann die Rindenstruktur der verschiedenen Baumarten ein limitierender Faktor sein.

Das Einleiten von synthetischer Luft in die Absaugkammer anstelle des Luftfilters hat sich bei einer großen Anzahl $(\mathrm{N}>5)$ zu beprobender Stämme im Freiland aufgrund der Kosten und des zusätzlichen Gewichtes der Gasflaschen als nicht umsetzbar herausgestellt. Zudem hat der Dichtigkeitsversuch gezeigt, dass der Luftfilter bereits den Hauptteil von Fremdstoffen aus der Umgebungsluft abfängt.

Ein weiteres Einsatzgebiet für diese Methode stellt neben der Volatilenmessung auch die Messung der $\mathrm{CO}_{2}$-Abgabe des Stammes dar. Mit geringem Adaptionsaufwand durch die Änderung der Probenahme mittels Omnifix ${ }^{\circledR} 10 \mathrm{ml}$ Spritzen, verbunden mit einem Omnifix ${ }^{\circledR}-2$ Zweiwegehahn (Firma B. Braun) und Anpassung der Teflonadapter an die 
Größe der Spritzenkanüle können ohne Weiteres $\mathrm{CO}_{2}$-Proben genommen werden.

Diese neue Stammabsaugkammer bietet mit ihrer Vereinfachung der Probennahme im Freiland die Möglichkeit, die bisher im Gegensatz zu den Blättern noch wenig erforschte Duftstoffquelle Stamm besser zu untersuchen. Diese Duftstoffquelle wird spätestens im Hinblick auf die Stressfrüherkennung (Trockenstress) für die prognostizierte Klimaentwicklung immer mehr in den Vordergrund treten [141. Mittels der Stammabsaugkammer kann ein Unterschied im Jahresverlauf der Duftstofffreisetzung aufgezeigt werden. Dies spricht für die Verwendung der Methode bei Fragestellungen, die den Verlauf der jahreszeitlichen Duftemission unter Berücksichtigung der saisonalen Pflanzenentwicklung und saisonal bedingten Stressfaktoren (Sommertrockenheit, Frost) untersuchen. Die durch die Stammabsaugkammer detektierbaren verändernden Duftmuster von Baumstämmen sind für Insekten Signale, die die Befallsdisposition des einzelnen Baumes aufzeigen können $[142$. 


\subsection{Kohlenstoffabgabe von Stämmen von $F$. sylvatica mittels VOC}

Über Photosynthese und Atmung geben Pflanzen Kohlenstoff (C) an die Atmosphäre ab. Einen geringeren, aber nicht vernachlässigbaren Anteil macht die Kohlenstoffabgabe der Pflanzen über die Freisetzung von kohlenstoffhaltigen Substanzen, den VOC, aus. Die bisherigen Untersuchungen zur Kohlenstoff-Freisetzung von Bäumen über VOC haben sich vorwiegend mit Blatt-VOC beschäftigt [126, 133, 143]. Die über die Blätter abgegebenen Kohlenwasserstoffe werden bis zu 88 \% über MT emittiert 127]. Das Wissen über den Kohlenstoff-Anteil, der im Laufe des Jahres über die Stammoberfläche abgegeben wird, kann dazu beitragen, die Berechnung der Kohlenstoff-Bilanz zu verbessern.

In dieser Studie soll überprüft werden, ob die Stammabsaugkammer zur Messung des Kohlenstoff-Anteils von VOC genutzt werden kann, und sie dient der Überprüfung der Einsatzmöglichkeiten der Stammabsaugkammer für verschiedene Forschungsbereiche.

\subsubsection{Material und Methoden}

Für die Messungen wurden sechs Stämme von F. sylvatica auf der Versuchsfläche Calvörde (Sand) ausgesucht. Detaillierte Informationen zu topographischen, klimatischen, edaphischen und bestandesstrukturellen Eigenschaften der Versuchsfläche sind unter Kapitel 2.5 aufgeführt. Die untersuchten Stämme wurden über vier Monate (August bis November 2010) mittels der oben beschriebenen Stammabsaugkammer unter Verwendung von Tenax ${ }^{\circledR}$ TA Röhrchen als Adsorbens beprobt. Die Temperatur und die Luftfeuchtigkeit an den Messtagen während der Beprobung sind in Tab. 2 aufgelistet.

Tabelle 2: Temperatur- und Luftfeuchtigkeitsdaten für den jeweiligen Messtag auf der Fläche Calvörde Sand

\begin{tabular}{|l|r|r|r|r|}
\hline Datum & 23.08 .2010 & 02.09 .2010 & 08.10 .2010 & 29.11 .2010 \\
\hline Temperatur & $20{ }^{\circ} \mathrm{C}$ & $17{ }^{\circ} \mathrm{C}$ & $16{ }^{\circ} \mathrm{C}$ & $-1{ }^{\circ} \mathrm{C}$ \\
\hline Luftfeuchtigkeit & $55 \%$ & $75 \%$ & $70 \%$ & $75 \%$ \\
\hline
\end{tabular}

\section{Analyse}

Die Tenax ${ }^{\circledR}$-Proben wurden durch einen GC (HP6890N, Agilent Technologies, Santa Clara, USA) mit einem gekoppelten quadrupolen MS (5973N, Agilent Technologies) mit EI Ionenquelle analysiert. Im GC befand sich eine unpolare HP-5MS Säule $(30 \mathrm{~m}$ x 0,2 mm ID x 0,24 $\mu \mathrm{m}$ Filmdicke, Agilent Technologies). Der GC war mit einem TDS A2 Thermodesorption Autosampler (Agilent Technologies) mit einem Kaltaufgabesystem (Cryofalle, CIS 4, Gerstel, Mühlheim, Deutschland) ausgestattet. Die VOC wurden vom Tenax ${ }^{\circledR}$ durch das Erhitzen bei $280{ }^{\circ} \mathrm{C}$ für drei Minuten im Thermodesorption System desorbiert. Das Trägergas, Helium (5.0; Air Liquid, Kornwestheim, Deutschland) mit ei- 
ner konstanten Flussrate von $40 \mathrm{ml} \mathrm{min}^{-1}$, beförderte die gesamte VOC-Probe auf das Kaltaufgabesystem, wo die VOC auf $-75{ }^{\circ} \mathrm{C}$ gekühlt wurden. Anschließend wurde die VOC-Probe durch schnelles Erhitzen auf $250{ }^{\circ} \mathrm{C}\left(12{ }^{\circ} \mathrm{C} \mathrm{s}^{-1}\right)$ verdampft und auf die unpolare Säule überführt. Das Ofenheizprogramm begann bei $40{ }^{\circ} \mathrm{C}$ und hielt diese für 3 min, um dann $7,5{ }^{\circ} \mathrm{C} \mathrm{min}^{-1}$ bis zum Erreichen der Endtemperatur von $280{ }^{\circ} \mathrm{C}$ hochzufahren. Die Endtemperatur wurde für 5 min gehalten. Helium (5.0; Air Liquid) diente im Ofen als Trägergas $\left(1 \mathrm{ml} \mathrm{min}^{-1}\right)$. Die Scanweite des MS war 11-300 amu.

\section{VOC-Auswahl}

Es wurden vier VOC ( $\alpha$-Pinen, $\beta$-Pinen, Camphen und 3-Caren) für die Berechnung des Kohlenstoff-Anteils ausgewählt. Alle vier VOC gehören zur Stoffklasse der MT und besitzen dieselbe Molmasse von 136,24 $\mathrm{g} \mathrm{mol}^{-1}$.

\section{Berechnung der Kohlenstoffmenge}

Die Peakflächen der ausgewählten VOC wurden im ,single ion modus“ (SIM) des Programmes Enhanced Data Analysis nach der relativ intensivsten Masse, in die ein VOC nach der Ionisierung am Massenspektrometer zerfällt, dem Hauption, integriert. Anhand von Standardmessungen von Reinsubstanzen in einer $10^{-2}$-Verdünnung (Dichlormethan/ Methanol-Lösung, 3:1, Merck) für $\beta$-Pinen und $\alpha$-Pinen und einer $10^{-4}$-Verdünnung (Dichlormethan/Methanol-Lösung, 3:1, Merck) für Camphen und 3-Caren konnte zu den ausgewählten vier VOC über eine Einpunkt-Kalibrierung die abgegebene Menge Kohlenstoff berechnet werden. Anschließend wurde die Kohlenstoffabgabe für jede Messung berechnet und das Ergebnis auf die gesamte Stammoberfläche unter der Annahme, dass die Emission über die gesamte Stammfläche gleich bleibt, hochgerechnet.

Beispiel zur Berechnung der abgegebenen Menge Kohlenstoff in ng:

$1 \mu \mathrm{l}$ der Standardsubstanz $\alpha$-Pinen in einer $10^{-2}$-Verdünnung ergeben nach dem Hauption 93 integrierte Peakfläche (im SIM) eine Abundanz von 381995912.

Die Abundanz des in der Probe vorkommenden $\alpha$-Pinens bei einer zweistündigen Messung ergibt nach Auswertung nach dem Hauption 93 eine Abundanz von 1275623.

Aus beiden Werten wird die Konzentration für $\alpha$-Pinen nach Gleichung 1 berechnet und beträgt für die Probe 0,033 nl.

$$
\begin{aligned}
\frac{1 \mu l * 10^{-2}}{\text { Abundan }_{\text {Standard }}} & =\frac{\text { Konzentration }_{\text {Probe }}}{\text { Abundan }_{\text {Probe }}} \\
\text { Konzentration }_{\text {Probe }} & =\frac{1 \mu l * 10^{-2}}{\text { Abundan }_{\text {Standard }}} * \text { Abundan } z_{\text {Probe }}
\end{aligned}
$$


Das Ergebnis wird mit der Dichte von $\alpha$-Pinen multipliziert, um die Masse in Kohlenstoff für $\alpha$-Pinen aus einer zweistündigen Messung zu erhalten (Gleichung 2). $\alpha$-Pinen hat bei Raumtemperatur eine Dichte von $0,86 \mathrm{~g} \mathrm{~cm}^{-3}$. Dies ergibt für $\mathrm{m}_{\alpha-\text { Pinen }} 287 \mathrm{ng}$.

$$
m=D * \text { Konzentration }_{\text {Probe }}
$$

$$
\begin{array}{lrl}
\text { mit: } & m- & \text { Masse, } \\
& D- & \text { Dichte, } \\
\text { Konzentration }_{\text {Probe }}- & \text { Konzentration der Probe. }
\end{array}
$$

Die Strukturformel von $\alpha$-Pinen lautet $\mathrm{C}_{10} \mathrm{H}_{16}\left(=136 \mathrm{~g} \mathrm{~mol}^{-1}\right)$. Von diesen $136 \mathrm{~g} \mathrm{~mol}^{-1}$ macht Kohlenstoff $88 \%$ aus und somit auch von den $287 \mathrm{ng}$. Das bedeutet, dass ein Baum innerhalb der zweistündigen Messung durch das VOC $\alpha$-Pinen 253 ng Kohlenstoff in die Stammabsaugkammer abgegeben hat.

\section{$\underline{\text { Berechnung der Kohlenstoffabgabe über den gesamten Stamm: }}$}

Es wird angenommen, dass der Stamm ein Kegelstumpf ist, so dass die Emissionsfläche „Stamm“ über die Mantelfäches des Kegels berechnet werden kann. Mittels der Formel für die Berechnung eines Kegelstumpfes lässt sich die Mantelfläche des Stammes $\left(\mathrm{M}_{\text {Stamm }}\right)$ anhand der Gleichung 3 berechnen. Hierbei wird die Ausbauchungsreihe von Schober [144] für F. sylvatica zur Hilfe genommen, die besagt, dass sich ein $22 \mathrm{~m}$ hoher Stamm am Ende auf $40 \%$ des Brusthöhendurchmessers (BHD) verjüngt. Daraus folgt $a_{B u c h e}=0,4$.

$$
\begin{aligned}
M_{\text {Stamm }}=(R+r) * \pi * m & \\
= & \left(\frac{B H D}{2}+\frac{a_{\text {Buche }} * B H D}{2}\right) * \pi * \sqrt{\frac{B H D}{2}-\left(\frac{a_{\text {Buche }} * B H D}{2}\right)^{2}+h^{2}} \\
\text { mit: } \quad & \text { Mantelfäche Stamm, } \\
& M_{\text {Stamm }}-\quad \text { Radius der Grundfläche, } \\
R- & \text { Radius der Deckfläche eines Kegelstumpfes, } \\
r- & \text { Länge einer Mantelfläche, } \\
m- & \text { Brusthöhendurchmesser, } \\
B H D- & \text { Ausbauchungsfaktor der Buche }(=0,4), \\
a_{\text {Buche }}- & \text { Höhe. }
\end{aligned}
$$

Für einen mittleren Baum auf der Versuchsfläche mit $25 \mathrm{~cm}$ BHD und $22 \mathrm{~m}$ Höhe ergibt sich darüber $M_{\text {Stamm }}$ von $12,10 \mathrm{~m}^{2}$. 
Bei der Gleichung zur Berechnung der Mantelfäche, die durch den Bratschlauch am Stamm abgedeckt wird ( $\left.M_{\text {Bratschlauch }}\right)$, wird angenommen, dass der Stammabschnitt sich wie ein Zylinder verhält:

$$
M_{\text {Bratschlauch }}=\pi * B H D * h
$$

$$
\begin{aligned}
& d-\quad \text { Durchmesser (BHD), } \\
& h-\quad \text { Höhe, } \\
& M_{\text {Bratschlauch }}-\quad \text { Mantelfläsche Bratschlauch. }
\end{aligned}
$$

Für einen mittleren Baum auf der Versuchsfläche mit $25 \mathrm{~cm}$ BHD und einer Höhe der Stammabsaugkammer von $20 \mathrm{~cm}$ ergibt dies für $\mathrm{M}_{\text {Bratschlauch }} 1570,8 \mathrm{~cm}^{2}$.

Zur Berechnung der Kohlenstoff-Abgabe über die gesamte Fläche eines Stammes über ein ausgewähltes VOC wurde die Gleichung 5 verwendet. Dabei beziehen sich $C_{\text {Stamm }}$ und $C_{\text {Bratschlauch }}$ auf die Kohlenstoffmenge, die innerhalb der zweistündigen Messungen über ein ausgewähltes VOC emittiert wird.

$$
C_{\text {Stamm }}=C_{\text {Bratschlauch }} * \frac{M_{\text {Stamm }}}{M_{\text {Bratschlauch }}}
$$

Bei der Annahme, dass ein Baum auf seiner gesamten Stammoberfläche gleich stark das jeweilige VOC emittiert, ergibt sich für das Beispiel-VOC $\alpha$-Pinen eine Kohlenstoffmenge von $1,95 * 10^{-5} \mathrm{~g}$, die über die Stammoberfläche eines $22 \mathrm{~m}$ hohen Stamms von F. sylvatica emittiert wird.

Die statistische Auswertung wurde mit R Studio (Version 3.1.1, The R Foundation for Statistical Computing) durchgeführt. Um Unterschiede in der Kohlenstoffabgabe zwischen den einzelnen Messterminen zu berechnen, wurde, da die Voraussetzung für einen parametrischen Test (Normalverteilung) nicht gegeben war, der Wilcoxon-Test mit BonferroniKorrektur verwendet. Das Signifikanzniveau lag bei $\alpha=0,013$. 


\subsubsection{Ergebnisse}

In Abb. 6] sind die berechneten Kohlenstoffmengen für die vier VOC ( $\alpha$-Pinen, $\beta$-Pinen, Camphen und 3-Caren), die am jeweiligen Messtag in den Monaten August bis November gemessen wurden, in ng angegeben. Trotz Minusgraden im November (Tab. 2) konnte ein Kohlenstofffluss auf der Versuchsfläche Calvörde Sand nachgewiesen werden. $\beta$-Pinen $(\mathrm{p}=0,004)$ zeigt eine signifikant geringere Kohlenstoffabgabe im November als im Sommermonat August (Abb. 6). 3-Caren konnte in der Messung im November auf der Fläche Calvörde Sand nicht nachgewiesen werden.

$\alpha$-Pinen $(\mathrm{m} / \mathrm{z})=93$

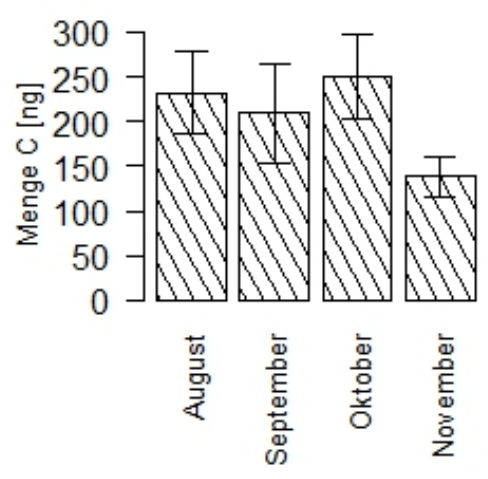

Camphen $(\mathrm{m} / \mathrm{z})=93$

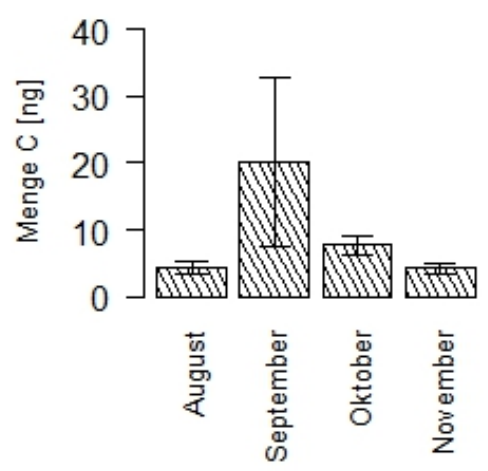

$\beta$-Pinen $(\mathrm{m} / \mathrm{z})=93$

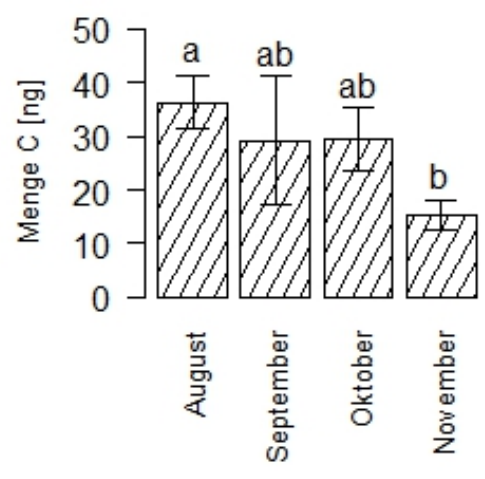

3-Caren $(\mathrm{m} / \mathrm{z})=107$

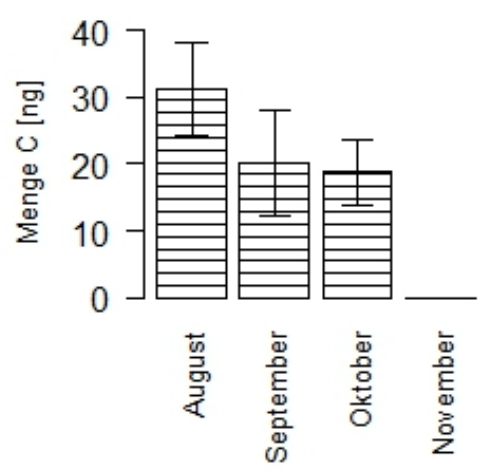

Abbildung 6: Kohlenstoffabgabe von Stämmen von F. sylvatica. Hier dargestellt die Kohlenstoffabgabe (Mittelwerte \pm s. e.) von vier VOC bei einer zweistündigen Beprobung an dem jeweiligen Messtag im August, September, Oktober und November 2010 auf der Fläche Calvörde (Sand). Verschiedene Buchstaben geben signifikante Unterschiede in der Kohlenstoffabgabe zwischen den Messtagen wieder (Wilcoxon-Test mit Bonferroni-Korrektur, $\mathrm{p} \leq 0,013$ ) 
In der Abb. 7 sind die Kohlenstoffmengen für alle vier VOC als Mittelwert in ng für die jeweilige Messung in dem entsprechendem Monat bezogen auf die Stammoberfläche eines $22 \mathrm{~m}$ hohen Stamms von F. sylvatica mit $25 \mathrm{~cm}$ BHD dargestellt.

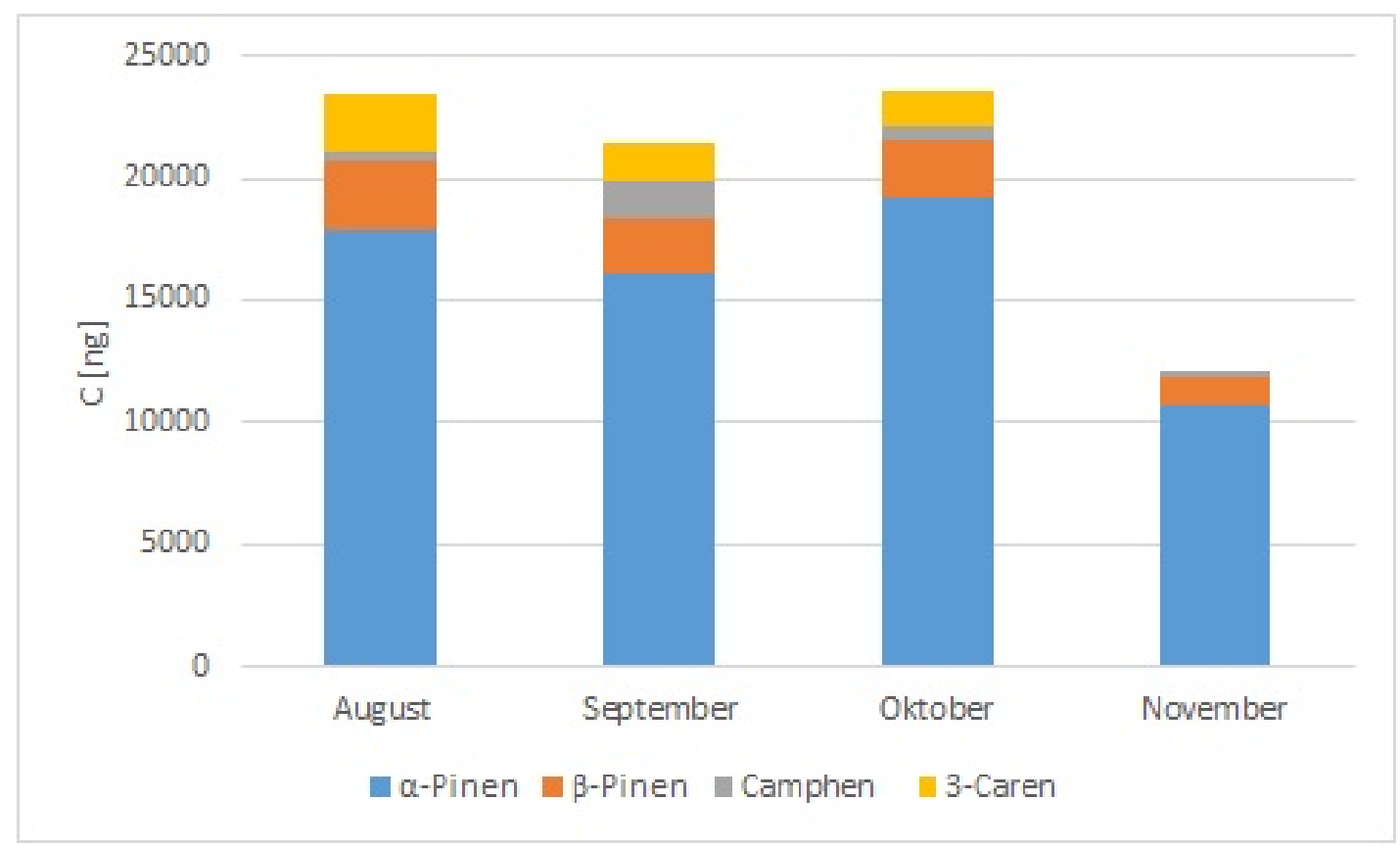

Abbildung 7: Kohlenstoffabgabe der vier VOC für die gesamte Stammoberfläche von $F$. sylvatica auf der Fläche Calvörde (Sand). Hier dargestellt die Mittelwerte aus sechs Messungen $(2 \mathrm{~h})$ der jeweiligen vier VOC

Unter der Annahme, dass ein Hektar mit 600 Bäumen von F. sylvatica bestockt ist (im Mittel $22 \mathrm{~m}$ hoch mit einem BHD von $25 \mathrm{~cm}$ ), emittiert dieser Hektar innerhalb der zwei Messstunden aufgrund des VOC $\alpha$-Pinen an dem Messnachmittag im August 0,01 g Kohlenstoff und aufgrund aller vier untersuchten VOC 0,014 g über die Stammoberflächen. 


\subsubsection{Diskussion}

In dieser Studie wurden vier VOC ( $\alpha$-Pinen, $\beta$-Pinen, Camphen und 3-Caren) aus der Stoffgruppe der MT ausgewählt. MT sind die am meisten studierten VOC-Emissionen des Laubes, da sie einen großen Teil zu den globalen Kohlenstoffemissionen beitragen und sehr reaktiv in der Atmosphäre sind 145,146. Global machen die jährlichen natürlichen VOC-Flüsse aus biogenen Quellen schätzungsweise bis zu $1150 \mathrm{Tg}$ Kohlenstoff aus, wovon etwa $11 \%$ aus MT bestehen [145. Zudem tragen MT zur Ozonchemie und zur Partikelbildung bei und können durch den Klimawandel einen indirekten Einfluss auf die Atmosphärenchemie haben 146.

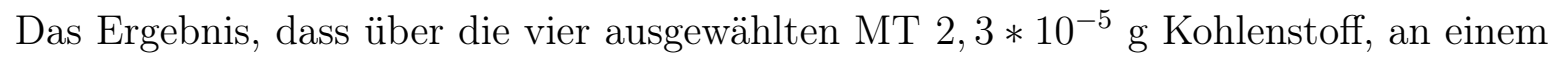
Nachmittag im August innerhalb von zwei Stunden über die Stammoberfläche eines $22 \mathrm{~m}$ hohen Stamms (BHD $25 \mathrm{~cm}$ ) von F. sylvatica emittiert werden, wirkt im Gegensatz zu dem, was schätzungsweise eine gleichgroße Buche an Kohlenstoff (109-218 kg) speichert [147, relativ gering. Doch ist nicht nur die Menge des Kohlenstoffes, die über ein VOC freigesetzt wird wichtig für dessen Einfluss auf das Klima, sondern auch welchen Anteil das jeweilige VOC bei der Bildung von sekundären organischen Aerosolen spielt. So trägt das VOC Isopren, die von Pflanzen am stärksten emittierte Verbindung (500 Tg C/Jahr) [145, nur einen geringen Teil zur Bildung von sekundären organischen Aerosolen bei [148. MT und besonders Sesquiterpene haben dagegen ein großes Potential zur Bildung von sekundären organischen Aerosolen [149.

In einer Untersuchung von Ceschia et al. (2002) gibt ein zwischen 25-35 Jahre alter Buchenstamm in Brusthöhe 0,21 $\mu \mathrm{mol} \mathrm{m} \mathrm{m}^{-2} \mathrm{~s}^{-1} \mathrm{C}$ über die Stammoberfläche durch Stammatmung ab [150]. Rechnet man das Ergebnis, was ein Stamm in dieser Arbeit im August freisetzt in die Einheit $\mu \mathrm{mol} \mathrm{m}{ }^{-2} \mathrm{~s}^{-1} \mathrm{C}$ um, so wird über alle vier MT 0,0017 $\mu \mathrm{mol} \mathrm{m} \mathrm{m}^{-2}$ $\mathrm{s}^{-1} \mathrm{C}$ freigesetzt. Davon wird im August $0,0013 \mu \mathrm{mol} \mathrm{m} \mathrm{m}^{-2} \mathrm{~s}^{-1} \mathrm{C}$ nur vom VOC $\alpha$-Pinen allein über die Stammoberfläche emittiert.

Des Weiteren deckt sich die in dieser Studie gemessene erhöhte Terpen-Freisetzung im Monat August mit der Untersuchung von Holzke et al. (2006), wonach MT-Emissionen von Zweigen von F. sylvatica im Sommer hoch sind und erst im späteren Verlauf des Sommers im September abnehmen 126.

Es konnte in dieser Studie zudem gezeigt werden, dass es mit der Stammabsaugkammer möglich ist den Kohlenstofffluss von Stämmen zu messen. Die Berechnung der emittierten Kohlenstoffmenge erfolgte unter der Annahme, dass Bäume über ihre gesamte Stammoberfläche gleich stark VOC emittieren. Allerdings kann davon ausgegangen werden, dass die oberen weniger stark verholzten Stammteile mehr VOC abgeben. Aufgrund dessen, dass hier nur der untere Stammbereich beprobt wurde, ist anzunehmen, dass die hier ermittelten Kohlenstoffwerte der jeweiligen VOC in Wirklichkeit für den gesamten Stamm 
etwas höher sind.

Die Ergebnisse dieser Studie zeigen, dass Stämme von F. sylvatica mittels der Freisetzung von Terpenen als eine Quelle für Kohlenstoff in der Atmosphäre angesehen werden können und in der Berechnung der Modelle zum Kohlenstoff-Kreislauf berücksichtigt werden sollten. 


\subsection{Stamm-VOC-Messung entlang eines Niederschlagsgradienten}

Bisherige Untersuchgen zum VOC-Muster von F. sylvatica befassten sich mit dem VOCMuster von Blättern und Jungbuchen (Anhang Tab. A.1). Durch die Entwicklung einer Stammabsaugkammer in Leichtbauweise ist es möglich das VOC-Muster von F. sylvatica an Altbuchen mit geringem Zeit- und Arbeitsaufwand zu untersuchen.

Die Zielsetzung dieser Untersuchung ist die Identifizierung von Marker-VOC für trockengestresste F. sylvatica für eine verletzungsfreie Erkennung von durch Stress geschwächte Bestände. Im Untersuchungsjahr herrschte keine außergewöhnliche Sommertrockenheit wie 2003 und 2018, so dass der hier definierte Trockenstress sich auf die durchschnittliche jährliche Niederschlagsmenge von unter $600 \mathrm{~mm}$ bezieht.

\subsubsection{Material und Methoden}

Die Stamm-VOC-Messungen von F. sylvatica wurden im Jahr 2011 in drei Untersuchungsgebieten (Unterlüß, Göhrde, Calvörde) durchgeführt. Pro Untersuchungsgebiet wurde eine Versuchsfläche auf einem durch Lehm und einem durch Sand geprägten Boden ausgewählt. Die Versuchsflächen auf Sandböden waren immer schlechter mit Grundwasser versorgt als die Vergleichsflächen auf Lehmböden (s. Kapitel 2.5, Tab. 1).

Alle Untersuchungsgebiete unterscheiden sich aufgrund ihrer jährlichen Niederschlagsmenge. Die langjährige Niederschlagsmenge nimmt mit zunehmender Kontinentalität vom östlichen Niedersachsen zum westlichen Sachsen-Anhalt ab (766-544 mm). Dagegen nimmt das langjährige Temperaturmittel langsam von 8,5 auf $9,2{ }^{\circ} \mathrm{C}$ zu. Weitere edaphische und bestandesstrukturelle Charakteristika der Versuchsflächen sind in der Tab. 1 aufgeführt.

Im Untersuchungsjahr 2011 nahm die jährliche Niederschlagsmenge von Unterlüß über Göhrde zu Calvörde ebenfalls ab. Wobei sich die jährlichen Niederschlagsmengen zwischen den jeweiligen Lehm- und Sandflächen ähnlich waren (Tab. 1). Temperatur und Luftfeuchtigkeit an den jeweiligen Messtagen sind in Tab. 3 aufgelistet. Während der Messtage gab es bei den Untersuchungsgebieten zwischen den jeweiligen Versuchsflächen (Sand und Lehm) keine großen Unterschiede zwischen den gemessenen Temperaturen und Luftfeuchtigkeiten. 
Tabelle 3: Temperatur und Luftfeuchtigkeit [\%] an den Messtagen

\begin{tabular}{|c|c|c|c|c|c|c|c|c|c|}
\hline \multicolumn{10}{|c|}{ Temperatur } \\
\hline Standort & Bodentyp & Mai & Juni & Juli & Aug. & Sep. & Okt. & Nov. & Dez. \\
\hline \multirow{2}{*}{ Unterlüß } & Sand & $19^{\circ} \mathrm{C}$ & $21^{\circ} \mathrm{C}$ & $22{ }^{\circ} \mathrm{C}$ & $19{ }^{\circ} \mathrm{C}$ & $15^{\circ} \mathrm{C}$ & n. a. & $8^{\circ} \mathrm{C}$ & $7{ }^{\circ} \mathrm{C}$ \\
\hline & Lehm & $19{ }^{\circ} \mathrm{C}$ & $21{ }^{\circ} \mathrm{C}$ & $22{ }^{\circ} \mathrm{C}$ & $18{ }^{\circ} \mathrm{C}$ & $15^{\circ} \mathrm{C}$ & n. a. & $8^{\circ} \mathrm{C}$ & n. a. \\
\hline \multirow{2}{*}{ Göhrde } & Sand & $17^{\circ} \mathrm{C}$ & $20{ }^{\circ} \mathrm{C}$ & $19^{\circ} \mathrm{C}$ & $23^{\circ} \mathrm{C}$ & $16^{\circ} \mathrm{C}$ & $8^{\circ} \mathrm{C}$ & n. a. & $3{ }^{\circ} \mathrm{C}$ \\
\hline & Lehm & $17^{\circ} \mathrm{C}$ & $20{ }^{\circ} \mathrm{C}$ & $19{ }^{\circ} \mathrm{C}$ & $23{ }^{\circ} \mathrm{C}$ & $16{ }^{\circ} \mathrm{C}$ & $8^{\circ} \mathrm{C}$ & n. a. & $3{ }^{\circ} \mathrm{C}$ \\
\hline \multirow{2}{*}{ Calvörde } & Sand & $14^{\circ} \mathrm{C}$ & $26{ }^{\circ} \mathrm{C}$ & $22{ }^{\circ} \mathrm{C}$ & $24{ }^{\circ} \mathrm{C}$ & n. a. & $12{ }^{\circ} \mathrm{C}$ & $8^{\circ} \mathrm{C}$ & n. a. \\
\hline & Lehm & $13^{\circ} \mathrm{C}$ & $25{ }^{\circ} \mathrm{C}$ & $22{ }^{\circ} \mathrm{C}$ & $24{ }^{\circ} \mathrm{C}$ & n. a. & $12{ }^{\circ} \mathrm{C}$ & $9{ }^{\circ} \mathrm{C}$ & n. a. \\
\hline \multicolumn{10}{|c|}{ Luftfeuchtigkeit } \\
\hline Standort & Bodentyp & Mai & Juni & Juli & Aug. & Sep. & Okt. & Nov. & Dez. \\
\hline \multirow{2}{*}{ Unterlüß } & Sand & $70 \%$ & $53 \%$ & $66 \%$ & $69 \%$ & $70 \%$ & n. a. & $87 \%$ & $90 \%$ \\
\hline & Lehm & $74 \%$ & $54 \%$ & $65 \%$ & $78 \%$ & $74 \%$ & n. a. & $85 \%$ & n. a. \\
\hline \multirow{2}{*}{ Göhrde } & Sand & $38 \%$ & $55 \%$ & $68 \%$ & $61 \%$ & $66 \%$ & $76 \%$ & n. a. & $86 \%$ \\
\hline & Lehm & $40 \%$ & $55 \%$ & $68 \%$ & $56 \%$ & $66 \%$ & $79 \%$ & n. a. & $88 \%$ \\
\hline \multirow{2}{*}{ Calvörde } & Sand & $80 \%$ & $45 \%$ & $48 \%$ & $80 \%$ & n. a. & $71 \%$ & $70 \%$ & n. a. \\
\hline & Lehm & $83 \%$ & $48 \%$ & $48 \%$ & $81 \%$ & n. a. & $69 \%$ & $67 \%$ & n. a. \\
\hline
\end{tabular}

n. a. = nicht abrufbar

Nach dem Blattaustrieb von F. sylvatica im Mai wurde damit begonnen, soweit es die Wetterverhältnisse zugelassen haben, in jedem Monat Stamm-VOC-Messungen von F. sylvatica in den Untersuchungsgebieten Unterlüß, Göhrde und Calvörde und den dazugehörigen Versuchsflächen auf Sand- und Lehmboden durchzuführen. Pro Versuchsfläche wurden zufallsbedingt sieben Bäume ausgewählt, die in den darauffolgenden Monaten auf ihr Stamm-VOC-Muster mit der zuvor beschriebenen Stammabsaugkammer [129] (s. Kapitel 3.2 beprobt wurden. Die VOC-Proben wurde immer in der Nachmittagszeit (14-16 Uhr) in BHD-Höhe (1,3 m) der Bäume genommen. Die Messungen wurden aufgrund der nicht wasserfesten Messgeräte an regenfreien Tagen durchgeführt. Die Analyse der Proben erfolgte wie im Kapitel 3.3.1 beschrieben mittels eines GC-MS.

\section{VOC-Auswahl}

Alle mithilfe des Programm „ChemStation“ erfassten VOC wurden für jedes Untersuchungsgebiet in einer Häufigkeitstabelle zusammengefasst. Es wurden nur VOC berücksichtigt, die bei mindestens einem Aufnahmezeitpunkt in mehr als der Hälfte der Proben $(\mathrm{N}>3)$ auf einer der sechs Versuchsflächen vorkamen.

Für die jeweiligen VOC wurde der zugehörige Retentionsindex (RI) mit der Formel des modifizierten Kovats-Index berechnet (Gleichung 6] [151]. Dieser bezieht das Retentionsverhalten der jeweiligen VOC einer Probe auf die homologe Reihe der Alkane und gibt somit die Lage eines VOC relativ zu zwei benachbarten Alkan-Paaren an. Für die Berechnung des Kovats-Index wurden Relativmessungen mit Alkanstandards in Hexan (C8-C20, Sigma-Aldrich, Darmstadt, Deutschland) durchgeführt. 


$$
R I=100 *\left[n+(N-n) \frac{t_{r(\text { Volatile })}-t_{r(n)}}{t_{r(N)}-t_{r(n)}}\right]
$$

mit:

$$
\begin{array}{ll}
R I- & \text { Kovats Retentionsindex, } \\
n- & \text { Anzahl der Kohlenstoffatome des ersten n-Alkans, } \\
N- & \text { Anzahl der Kohlenstoffatome des zweiten n-Alkans, } \\
t_{r}- & \text { Retentionszeit. }
\end{array}
$$

Der RI ist im Gegensatz zum RT ein konstanter Parameter für eine bestimmte Säule und ein jeweiliges Temperaturprogramm. Dies ermöglicht die Überprüfung der passenden Zuordnung der VOC anhand der Datenbank des Analyseprogrammes zum jeweiligen Peak durch einen Abgleich der berechneten RI. Dafür wurden die berechneten RI mit den Indices aus der NIST Datenbank vom nationalen Institut für Standard und Technologie (NIST MS Search 2.0, Gaithersburg, USA) verglichen. Für den Abgleich wurden nur Indices von nicht-polaren Säulen mit einer ähnlichen Säulenlänge herangezogen. Die Zuordnung eines VOC wurde als passend eingestuft, wenn sich der berechnete RI von dem aus der Literatur nicht mehr als 50 Einheiten unterscheidet (Anhang A.3., A.4. und A.5.).

Peaks, denen kein VOC eindeutig zugewiesen werden konnte, wurden in den jeweiligen Häufigkeitstabellen mit „Unbekannt“ benannt. Alle VOC, die zugeordnet werden konnten, wurden für das jeweilige Untersuchungsgebiet in ihre chemischen Stoffklassen eingeordnet.

\section{Statistische Auswertung}

Im ersten Schritt der statistischen Auswertung wurde mittels einer multifaktoriellen ANOVA (analysis of variance) untersucht, ob die Stoffklassenverteilung (abhängige Variable) signifikant von den Einflussfaktoren Jahreszeit, Bodentyp (Sand, Lehm) oder Untersuchungsgebiet (Unterlüß, Göhrde, Calvörde) (unabhängige Variablen) abhängt. Die Daten erfüllten die Voraussetzung für die multifaktorielle ANOVA und die Modellresiduen waren normalverteilt. Folgende Nullhypothesen wurden untersucht:

- Keine Unterschiede zwischen dem durchschnittlichen Vorkommen der Stoffklassen und den drei Untersuchungsgebieten mit unterschiedlichen jährlichen Niederschlagsmengen.

- Keine Unterschiede zwischen dem durchschnittlichen Vorkommen der Stoffklassen und dem Bodentypen (Sand, Lehm).

- Keine Unterschiede zwischen dem durchschnittlichen Vorkommen der Stoffklassen und den Jahreszeiten. 
Die Monate Mai und Juni, Juli und August, September und Oktober sowie November und Dezember wurden als Frühling, Sommer, Herbst und Winter zusammengefasst. Die dazugehörigen Daten für das Vorkommen der Stoffklassen in den Proben wurden gemittelt und in \% angegeben. Die Zusammenfassung der Monate entspricht der Blattphänologie von F. sylvatica, die relativ spät im Jahr austreibt (von April bis Mai) [152], in den Sommermonaten (Juli und August) voll belaubt ist, deren Blätter sich im Herbst verfärben und abgeworfen werden (September und Oktober) und die im Winter (November und Dezember) unbelaubt ist.

Bei signifikanter Abhängigkeit der Stoffklassenverteilung von einzelnen Einflussfaktoren wurde im Anschluss der Bonferroni-Post-hoc-Test angewendet, um festzustellen, zwischen welchen Gruppen der jeweiligen Einflussfaktoren es zu signifikanten Unterschieden in der Stoffklassenverteilung kommt.

Im zweiten Schritt der Auswertung wurden aus der Stoffklasse der Terpene und der Aldehyde sechs pflanzenbürtige VOC ausgewählt und ihre Konzentrationen in den einzelnen Proben berechnet. Für die Berechnung der Konzentrationen wurden für Nonanal, $\alpha$-Pinen, Camphen und Decanal eine Vier-Punkt-Kalibrierungskurve und für Limonen und Campher eine Sechs-Punkt-Kalibrierungskurve verwendet und mittels linearer Geradenabschnittberechnung die dazugehörigen Konzentrationen ermittelt, wobei die Dichte des jeweiligen VOC mitberücksichtigt wurde.

Die Konzentrationsänderung innerhalb des Jahres wurde mit einer ANOVA berechnet und folgende Nullhypothesen überprüft:

- Keine Unterschiede in den Konzentrationen der untersuchten VOC innerhalb der verschiedenen Untersuchungsgebiete mit unterschiedlichen jährlichen Niederschlagsmengen.

- Keine Unterschiede in den Konzentration der untersuchten VOC zwischen den Bodentypen (Sand, Lehm).

- Keine Unterschiede in der Konzentration der untersuchten VOC im Verlauf des Jahres.

Im Anschluss wurde der Bonferroni-Post-hoc-Test für signifikante Unterschiede zwischen Konzentration und Jahreszeit und aufgrund der geringen Anzahl der Stichproben der Wilcoxon-Test für die Untersuchung von signifikanten Unterschieden der Konzentrationen zwischen den Bodentypen angewandt.

Die Auswertung wurde mit R Studio (Version 3.1.1) durchgeführt und das Signifikanzniveau lag bei allen Tests bei $\alpha=0,05$. 


\subsubsection{Ergebnisse}

\section{Stoffklassenzusammensetzung}

In allen drei Untersuchungsgebieten geben die Stämme von F. sylvatica auf den Versuchsflächen Sand und Lehm in den Sommermonaten Juli und August eine größere Anzahl an unterschiedlichen VOC ab als im Winter (Abb. 8, 9 und 10p. Insgesamt konnten den VOC elf Stoffklassen zugeordnet werden, wobei das Stamm-VOC-Muster durch vier Stoffgruppen (Terpene, Alkane, Aldehyde und Aromate) dominiert wird. Allerdings konnten nicht in jedem Untersuchungsgebiet VOC aus allen elf Stoffgruppen gemessen werden. In den Untersuchungsgebieten Göhrde und Calvörde konnten im Gegensatz zu Unterlüß keine organische Säure gemessen werden. In Göhrde wurde kein Acetat und in Calvörde kein Ether in den Stamm-VOC-Proben gefunden.

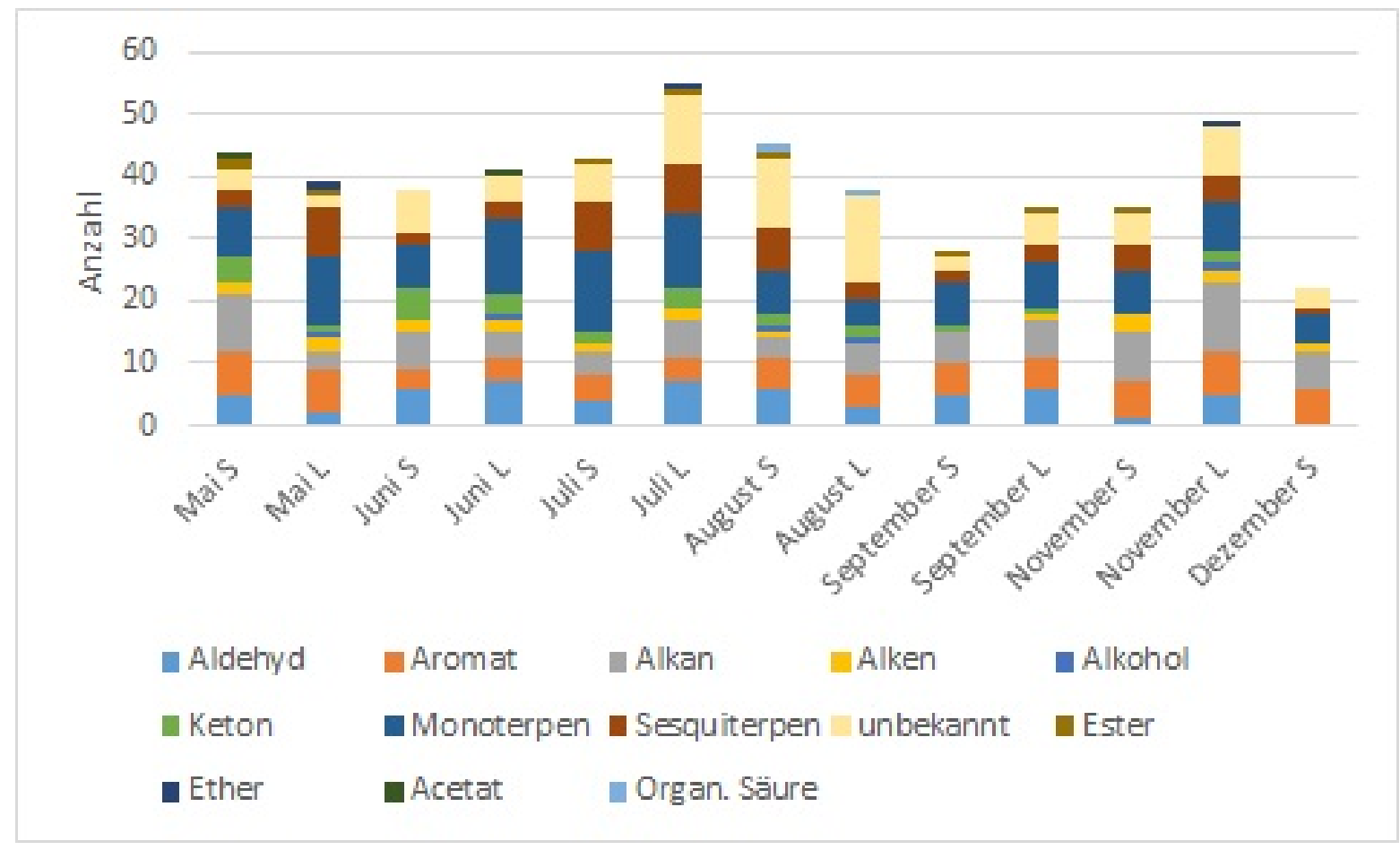

Abbildung 8: Anzahl der VOC über das Jahr 2011 bei Stammmessungen von F. sylvatica im Untersuchungsgebiet Unterlüß aufgeteilt nach Stoffgruppen. $[\mathrm{S}=$ Sand, $\mathrm{L}=$ Lehm 


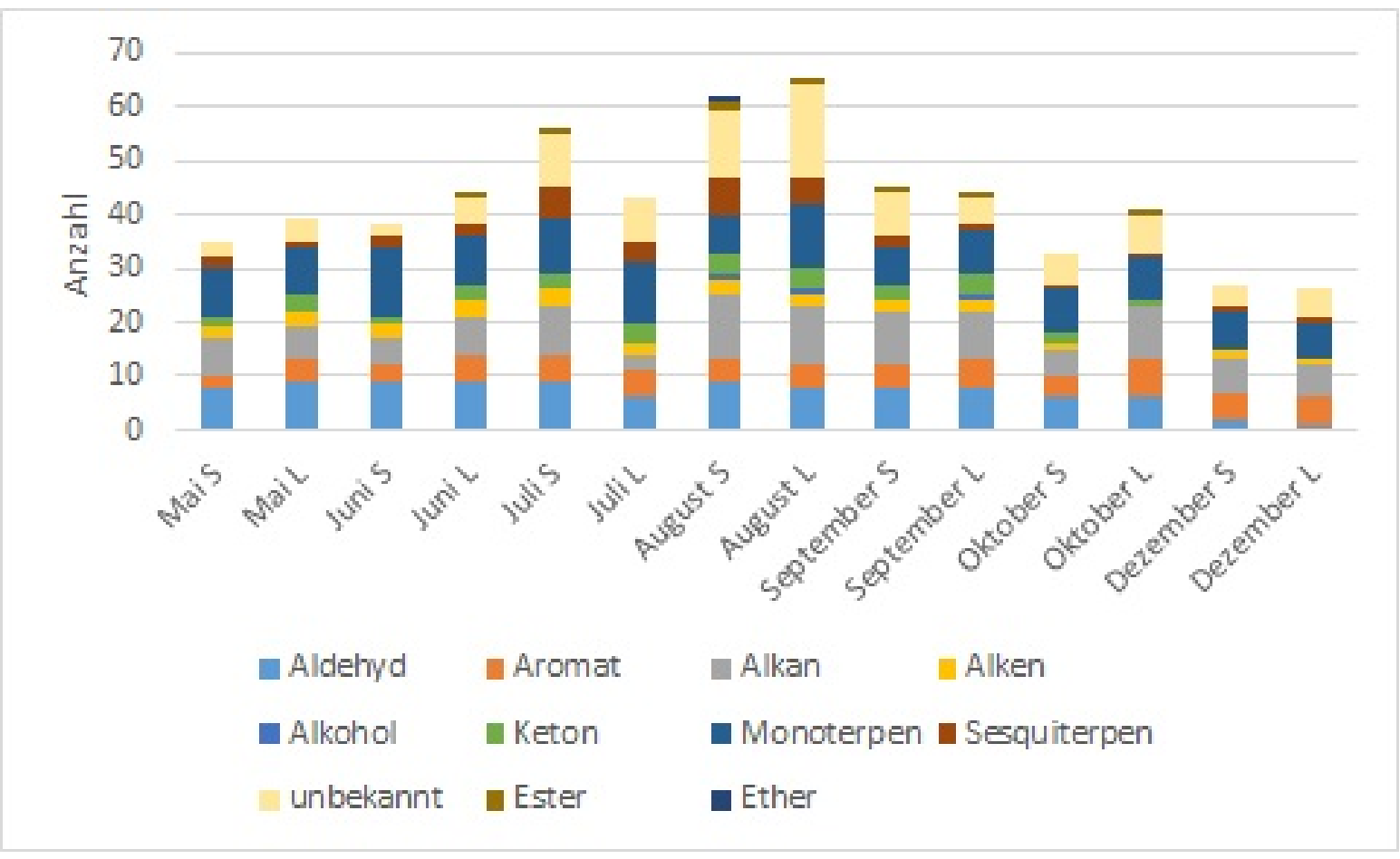

Abbildung 9: Anzahl der VOC über das Jahr 2011 bei Stammmessungen von F. sylvatica im Untersuchungsgebiet Göhrde aufgeteilt nach Stoffgruppen. S = Sand, L $=$ Lehm

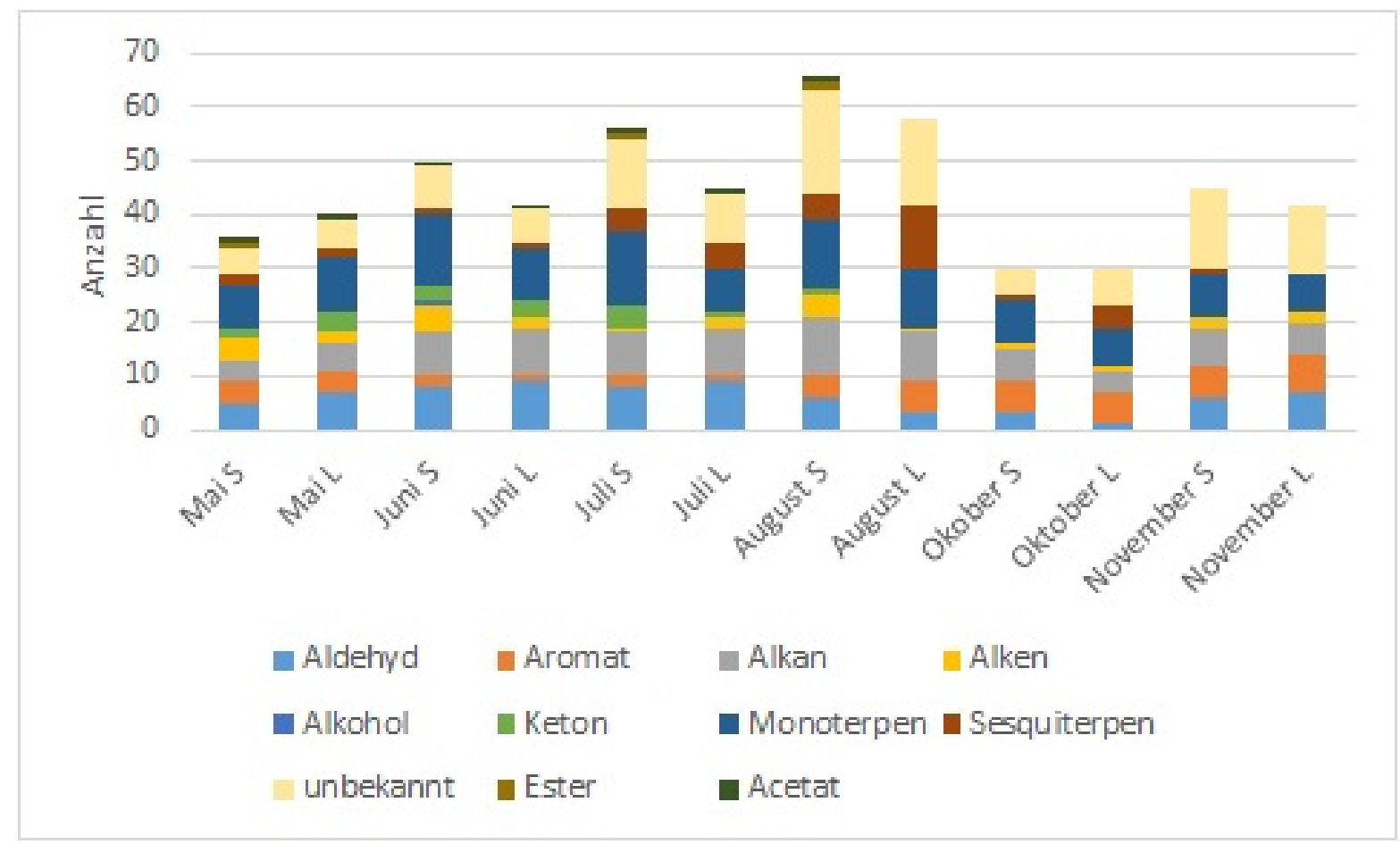

Abbildung 10: Anzahl der VOC über das Jahr 2011 bei Stammmessungen von F. sylvatica im Untersuchungsgebiet Calvörde aufgeteilt nach Stoffgruppen. S = Sand, $\mathrm{L}=\mathrm{Lehm}$ 
Bei Betrachtung der Stoffklassen fällt auf, dass mehr VOC aus der Stoffgruppe der Terpene, sowohl MT als auch Sesquiterpene ( $\underline{\mathrm{ST}})$, in den wärmeren Monaten vermehrt von den Stämmen abgegeben werden als in den Wintermonaten November und Dezember. Ähnlich verhält es sich bei den Aldehyden. Auch hier ist die Anzahl der verschiedenen VOC vorwiegend in den wärmeren Monaten größer als in den Wintermonaten. Die Anzahl der Alkane ist im Gegensatz zu Unterlüß im August in den Untersuchungsgebieten Calvörde und Göhrde auf beiden Versuchsflächen hoch, in Unterlüß dagegen im November. 


\section{Auswirkungen der jährlichen Niederschlagsmenge, des Bodentyps und der Jahreszeit auf das VOC-Vorkommen}

Das Vorkommen der Stoffklassen Aldehyde, Alkene, Aromate, Ketone, Terpene, Acetate und Unbekannt unterschied sich signifikant in den Jahreszeiten (Tab. 4. Abb. 11 und 12. Im Frühling kamen in den Proben signifikant mehr Aldehyde und Alkene vor als im Herbst. Ketone und Acetate wurden signifikant mehr in den Frühlingsproben gefunden als in den Herbst- und in Winterproben. Im Winter wurden signifikant mehr Aromate in den Proben nachgewiesen. Bei den Terpenen wurden signifikant mehr unterschiedliche VOC in den Proben im Frühjahr und im Sommer als in den Winterproben und bei den unbekannten VOC wurden signifikant mehr unterschiedliche VOC in den Sommerproben als zu den übrigen Jahreszeiten gefunden. Das Vorkommen der Stoffgruppen Alkene und Acetat unterschied sich zusätzlich signifikant abhängig von den jährlichen Niederschlagsmengen. Alkene wurden mehr in Göhrde-Proben nachgewiesen und das Vorkommen der Acetate war am geringsten in Calvörde-Proben.

Das Vorkommen der Alkohole und der Alkane wurde in den Proben von keinem der drei Faktoren beeinflusst (Tab. 4. Abb. 11).

Für die Stoffklassen Ether und organische Säure konnte aufgrund des geringen Vorkommens dieser Stoffklassen in den Proben keine ANOVA durchgeführt werden. Die organische Säure konnte nur in Proben von Unterlüß gefunden werden und wurde nur im Sommer sowohl auf der Sand- als auch auf der Lehmfläche in den Proben identifiziert. Ether konnte in Proben von Unterlüß und Göhrde gefunden werden. In Unterlüß kam Ether nur auf den Lehmflächen im Frühling, Sommer und Winter und in Göhrde auf der Sandfläche im Sommer vor.

Es gab keine Ausreißer und die Modellresiduen waren alle bis auf die Stoffgruppe Acetat normalverteilt.

Tabelle 4: Signifikante Unterschiede hinsichtlich des Vorkommens der Stoffklassen in Bezug auf die jeweiligen Einflussfaktoren

\begin{tabular}{|l|l|l|l|}
\hline \multicolumn{3}{|c|}{ p-Wert } \\
\hline Stoffgruppe & Untersuchungsgebiet & Bodentyp & Jahreszeit \\
\hline \hline Aldehyd & n. s. & n. s. & $0,018^{*}$ \\
\hline Alkan & n. s. & n. s. & n. s. \\
\hline Alken & $<0,001^{* * *}$ & n. s. & $0,001^{* * *}$ \\
\hline Alkohol & n. s. & n. s. & n. s. \\
\hline Aromat & n. s. & n. s. & $<0,001^{* * *}$ \\
\hline Ester & $0,026^{*}$ & $0,015^{* *}$ & $0,026^{*}$ \\
\hline Keton & n. s. & n. s. & $0,003^{* *}$ \\
\hline Terpen & $0,001^{* * *}$ & n. s. & $<0,001^{* * *}$ \\
\hline Acetat & $0,011^{* *}$ & n. s. & $0,005^{* *}$ \\
\hline Unbekannt & n. s. & n. s. & $<0,001^{* * *}$ \\
\hline
\end{tabular}

ANOVA: ${ }^{*} \mathrm{p} \leq 0,05, * * \mathrm{p} \leq 0,01, * * * \mathrm{p} \leq 0,001$, n. s. $=$ nicht signifikant 


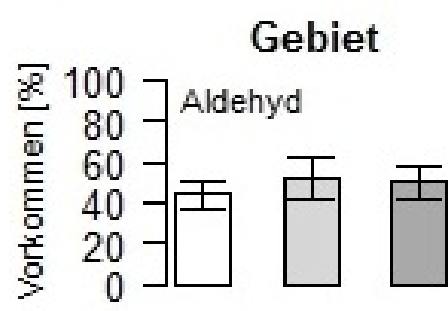

\section{Bodentyp}

Aldehyd
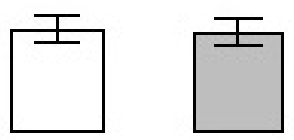

Alkan
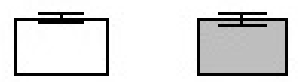

Alken
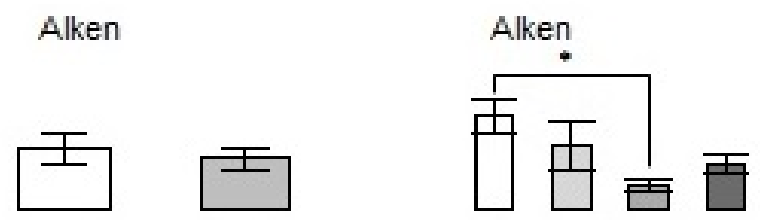

Alkohol

Alkohol

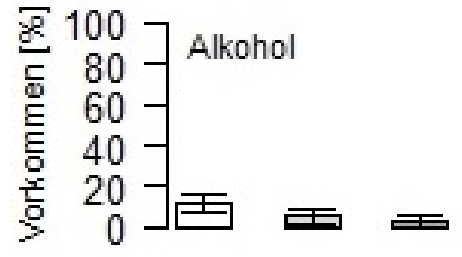

匠

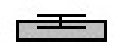

画

Aromat<smiles>C1#CCC1</smiles>

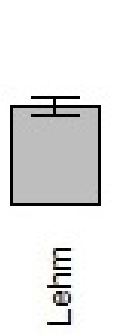

몸
Jahreszeit

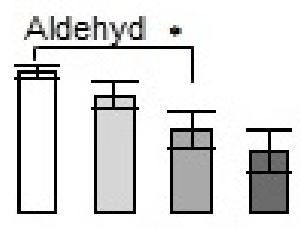

Alkan

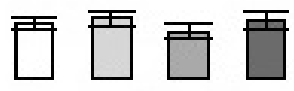

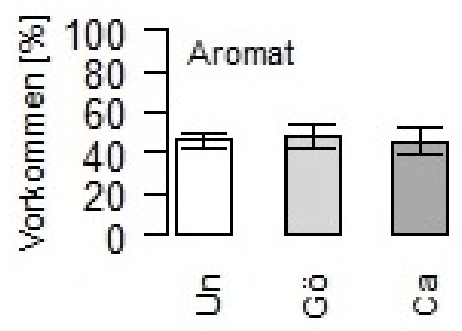

Aromat *

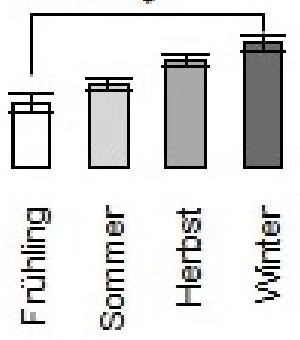

Abbildung 11: Vorkommen der Stoffklassen Aldehyd, Alkan, Alken, Alkohol und Aromat in allen genommenen Proben [\%] bei dem jeweiligen Untersuchungsgebiet, Bodentyp und Jahreszeit (Mittelwert \pm s. e.). Bonferroni-Test: ${ }^{*}=\mathrm{p} \leq$ $0,05,{ }^{* *}=\mathrm{p} \leq 0,01,{ }^{* * *}=\mathrm{p} \leq 0,001$. Un $=$ Unterlüß, Gö $=$ Göhrde, Ca $=$ Calvörde 


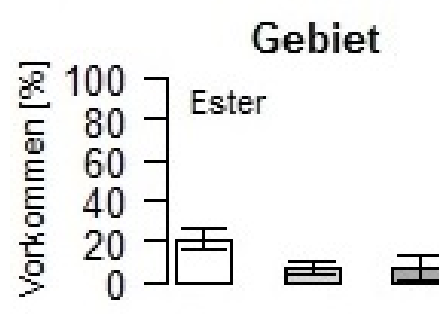

\section{Bodentyp}

Ester
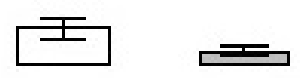

Keton
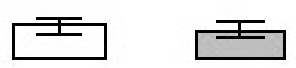

Terpen
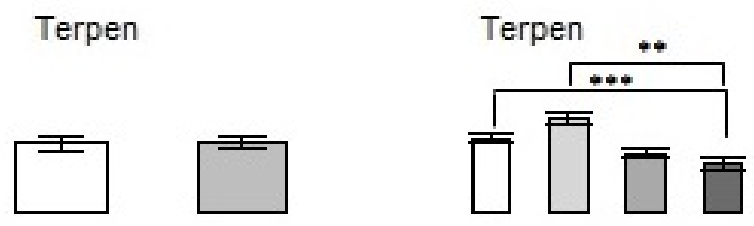
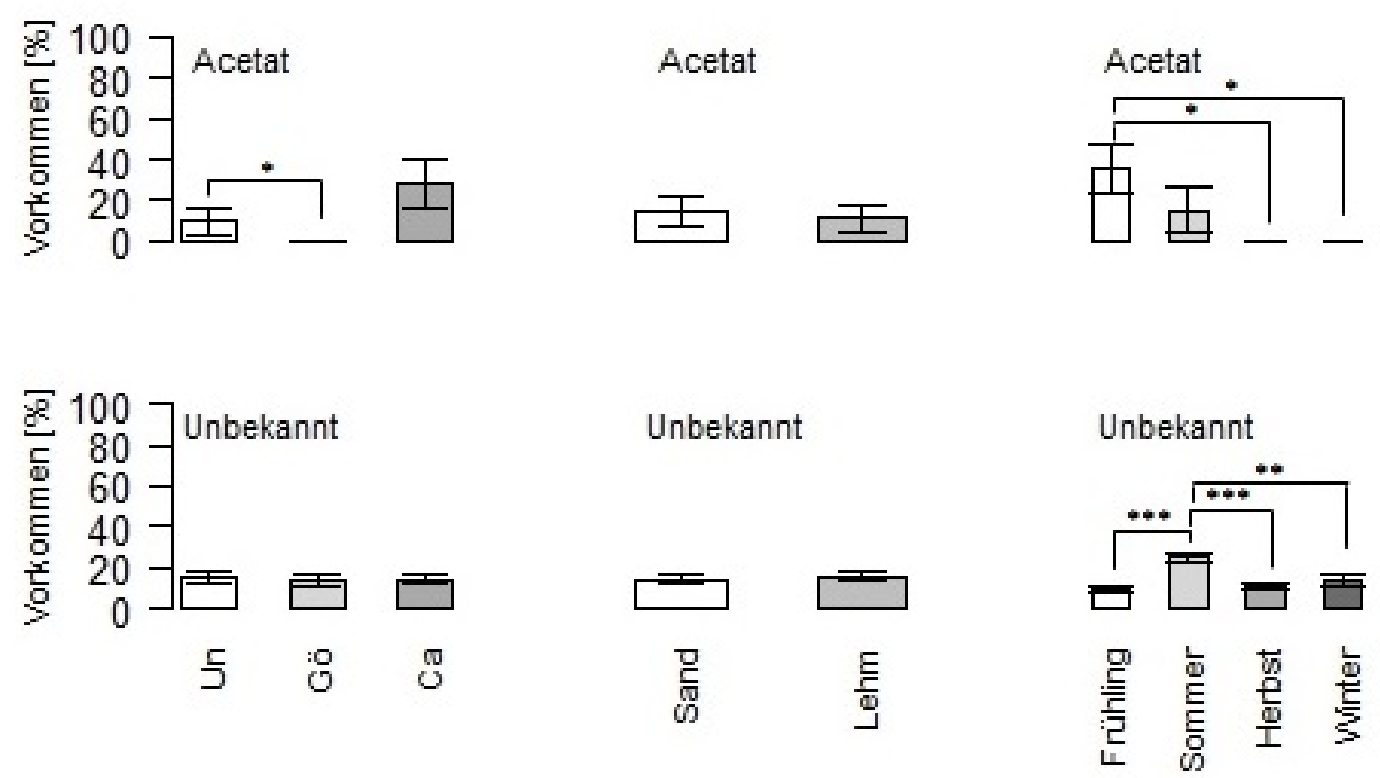

Abbildung 12: Vorkommen der Stoffklassen Ester, Keton, Terpen, Acetat und Unbekannt in allen genommenen Proben [\%] bei dem jeweiligen Untersuchungsgebiet, Bodentyp und Jahreszeit (Mittelwert \pm s. e.). Bonferroni-Test: ${ }^{*}=\mathrm{p} \leq$ $0,05,{ }^{* *}=\mathrm{p} \leq 0,01,{ }^{* * *}=\mathrm{p} \leq 0,001$. Un $=$ Unterlüß, Gö $=$ Göhrde, Ca $=$ Calvörde 


\section{Auswirkungen der jährlichen Niederschlagsmenge, des Bodentyp und der Jahreszeit auf die VOC-Konzentrationen}

Die ANOVA zeigte Auffälligkeiten für die Konzentrationen von fünf VOC ( $\alpha$-Pinen, Limonen, Nonanal, Decanal und Campher) bei der jährliche Niederschlagsmenge, dem Bodentyp und der Jahreszeit. Auf die Emissionsstärke von Camphen haben die drei Faktoren keinen Einfluss (Anhang Tab. A.5). Campher ist das einzige der untersuchten VOC, dessen Abgaberaten vom Stamm von F. sylvatica von allen drei Einflussfaktoren (jährliche Niederschlagsmenge, Bodentyp und Jahreszeit) beeinflusst wird (Anhang Tab. A.5. Die Abgabe der beiden Aldehyde Nonanal und Decanal hängt vom Bodentyp und der Jahreszeit ab. Die Menge Limonen, die vom Stamm abgegeben wird, hängt auch von der Jahreszeit und zusätzlich von der jährlichen Niederschlagsmenge ab. Dagegen wird die Abgabe von $\alpha$-Pinen nur von der jährlichen Niederschlagsmenge beeinflusst.

Für $\alpha$-Pinen zeigte der Post-hoc-Test signifikante Unterschiede zwischen dem Untersuchungsgebiet Unterlüß mit dem höchsten jährlichen Niederschlag zu den anderen beiden Untersuchungsgebieten. Auch Limonen und Campher zeigten signifikante Unterschiede für das Gebiet Unterlüß. Für Limonen lagen die Unterschiede zwischen Unterlüß und dem Untersuchungsgebiet mit den geringsten jährlichen Niederschlagsmengen und für Campher zwischen Unterlüß und dem Gebiet in der Mitte des Niederschlagsgradienten (Anhang Tab. A.6.).

Die Aldehyde zeigten in allen Untersuchungsgebieten signifikante jahreszeitliche Abhängigkeiten in der Abgaberate für mindestens einen Bodentyp (Abb. 13, Tab. 5). Dabei wird immer signifikant mehr Nonanal und Decanal im Frühjahr in den Untersuchungsgebieten emittiert und nimmt im Verlauf des Jahres zum Winter hin ab. Für das Untersuchungsgebiet Unterlüß sind diese Emissionsabnahmen für die Sandfläche signifikant, in Göhrde zeigen die Konzentrationen beider Aldehyde bei beiden Bodentypen eine signifikante Abhängigkeit zur Jahreszeit und in Calvörde, im Gebiet mit der geringsten jährlichen Niederschlagsmenge, ist dieser jahreszeitliche Trend signifikant für den Bodentyp Lehm.

Im Gegensatz zu den beiden Aldehyden konnte bei den Terpenen nur für einzelne Untersuchungsgebiete eine jahreszeitliche Abhängigkeit zur abgegebenen Konzentration bestimmt werden. Die Terpene $\alpha$-Pinen und Limonen haben gemeinsam, dass ihre Konzentration im Untersuchungsgebiet Calvörde auf dem Lehmstandort zum Herbst abnimmt und im Winter wieder leicht ansteigt (Abb. 14, Tab. 6). Limonen, Camphen und Campher zeigen im Untersuchungsgebiet Unterlüß im Laufe des Jahres einen abnehmenden Trend (Abb. 14 und 15, Tab. 6 und 7). Für Campher ist dieser Trend, sowohl auf dem Sand- als auch auf dem Lehmstandort signifikant, für Limonen nur auf dem Sandstandort.

Die Konzentrationen der Aldehyde zeigen die geringsten Unterschiede zwischen den jeweiligen Bodentypen (Abb. 13). Bei den Terpenen konnten für beide Bodentypen signi- 
fiknate Unterschiede nachgewiesen werden. Besonders unterschiedlich war die Abgaberate von $\alpha$-Pinen für die beiden Bodentypen im Untersuchungsgebiet Göhrde. Demnach wurde im Frühjahr, Sommer und Herbst signifikant mehr $\alpha$-Pinen von den Stämmen auf den Lehmstandorten emittiert. Im Winter konnte dagegen kein Unterschied in der Abgaberate für Göhrde festgestellt werden (Abb. 14). 


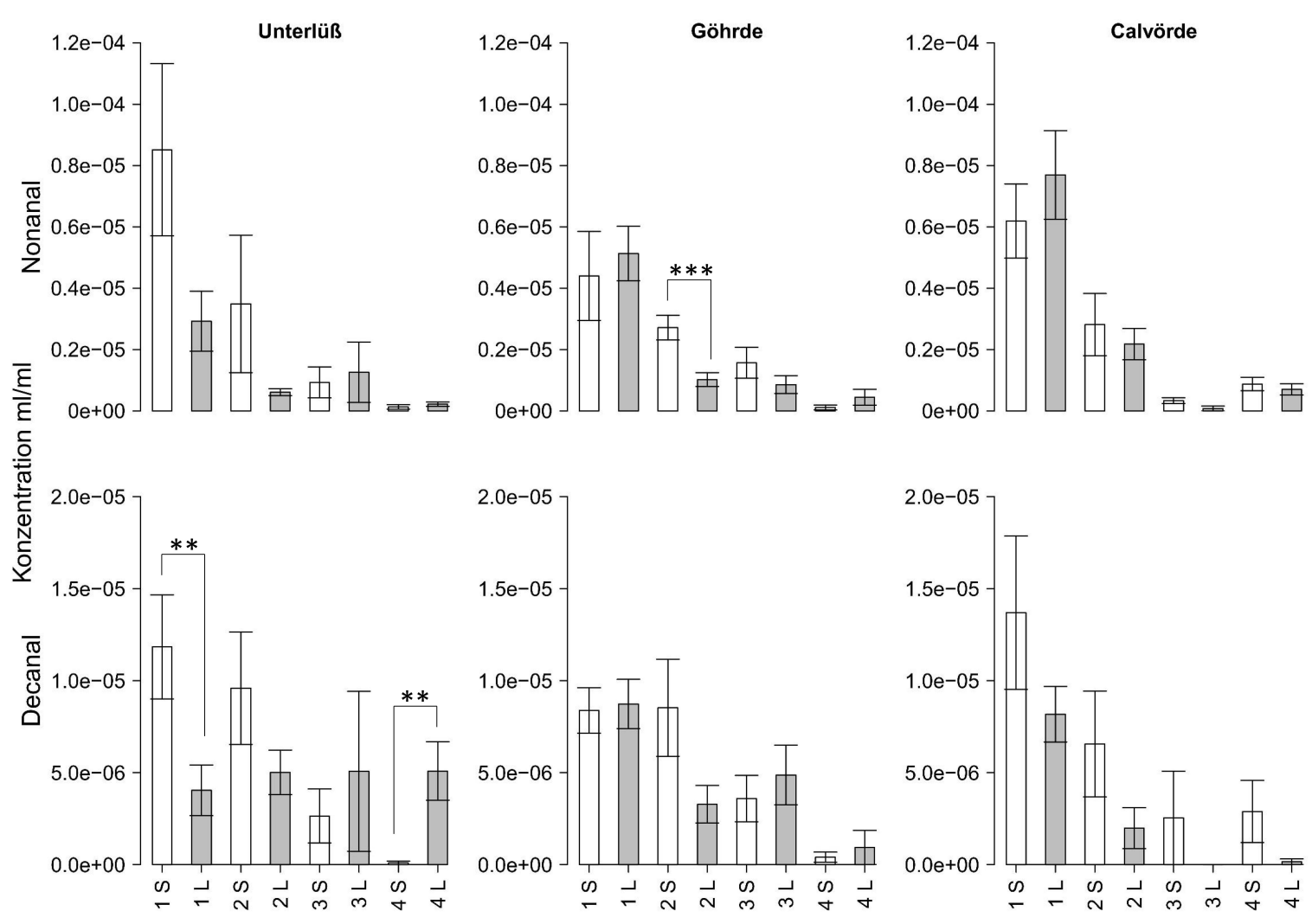

Abbildung 13: Emittierte Konzentrationen von Nonanal und Decanal innerhalb von zwei Stunden über einen Stamm von F. sylvatica zu unterschiedlichen Jahreszeiten und jährlichen Niederschlagsmengen $(\bar{x} \pm$ s. e.). Wilcoxon-Test: * $=\mathrm{p} \leq 0,05,{ }^{* *}=\mathrm{p} \leq 0,01,{ }^{* * *}=\mathrm{p} \leq 0,001 . \mathrm{S}=$ Sand, $\mathrm{L}=$ Lehm, $1=$ Frühjahr, $2=$ Sommer, $3=$ Herbst, $4=$ Winter

Tabelle 5: Jahreszeitliche Konzentrationsveränderungen von Nonanal und Decanal für den jeweiligen Bodentyp

\begin{tabular}{|l|c|c|c|c|c|}
\hline \multicolumn{7}{|c|}{ Nonanal } \\
\hline Standort & Bodentyp & Frühling & Sommer & Herbst & Winter \\
\hline \multirow{2}{*}{ Unterlüß } & $\mathrm{S}$ & $\mathrm{a}$ & $\mathrm{ab}$ & $\mathrm{ab}$ & $\mathrm{b}$ \\
\cline { 2 - 6 } & $\mathrm{L}$ & $\mathrm{a}$ & $\mathrm{a}$ & $\mathrm{a}$ & $\mathrm{a}$ \\
\hline \multirow{2}{*}{ Göhrde } & $\mathrm{S}$ & $\mathrm{a}$ & $\mathrm{ab}$ & $\mathrm{ab}$ & $\mathrm{b}$ \\
\cline { 2 - 6 } & $\mathrm{L}$ & $\mathrm{a}$ & $\mathrm{b}$ & $\mathrm{b}$ & $\mathrm{b}$ \\
\hline \multirow{2}{*}{ Calvörde } & $\mathrm{S}$ & $\mathrm{a}$ & $\mathrm{a}$ & $\mathrm{a}$ & $\mathrm{a}$ \\
\cline { 2 - 6 } & $\mathrm{L}$ & $\mathrm{a}$ & $\mathrm{b}$ & $\mathrm{b}$ & $\mathrm{b}$ \\
\hline \hline \multicolumn{6}{|c|}{ Decanal } \\
\hline Standort & Bodentyp & Frühling & Sommer & Herbst & Winter \\
\hline \multirow{2}{*}{ Unterlüß } & $\mathrm{S}$ & $\mathrm{a}$ & $\mathrm{a}$ & $\mathrm{ab}$ & $\mathrm{b}$ \\
\cline { 2 - 6 } & $\mathrm{L}$ & $\mathrm{a}$ & $\mathrm{a}$ & $\mathrm{a}$ & $\mathrm{a}$ \\
\hline \multirow{2}{*}{ Göhrde } & $\mathrm{S}$ & $\mathrm{a}$ & $\mathrm{a}$ & $\mathrm{ab}$ & $\mathrm{b}$ \\
\cline { 2 - 6 } & $\mathrm{L}$ & $\mathrm{a}$ & $\mathrm{b}$ & $\mathrm{ab}$ & $\mathrm{b}$ \\
\hline \multirow{2}{*}{ Calvörde } & $\mathrm{S}$ & $\mathrm{a}$ & $\mathrm{a}$ & $\mathrm{a}$ & $\mathrm{a}$ \\
\cline { 2 - 6 } & $\mathrm{L}$ & $\mathrm{a}$ & $\mathrm{b}$ & $\mathrm{b}$ & $\mathrm{b}$ \\
\hline
\end{tabular}

Unterschiedliche Buchstaben geben signifikante Unterschiede in den Konzentrationen zwischen den Jahreszeiten wieder (Bonferroni T-Test, $\mathrm{p} \leq 0,05$ ). $\mathrm{S}=$ Sand, $\mathrm{L}=$ Lehm 


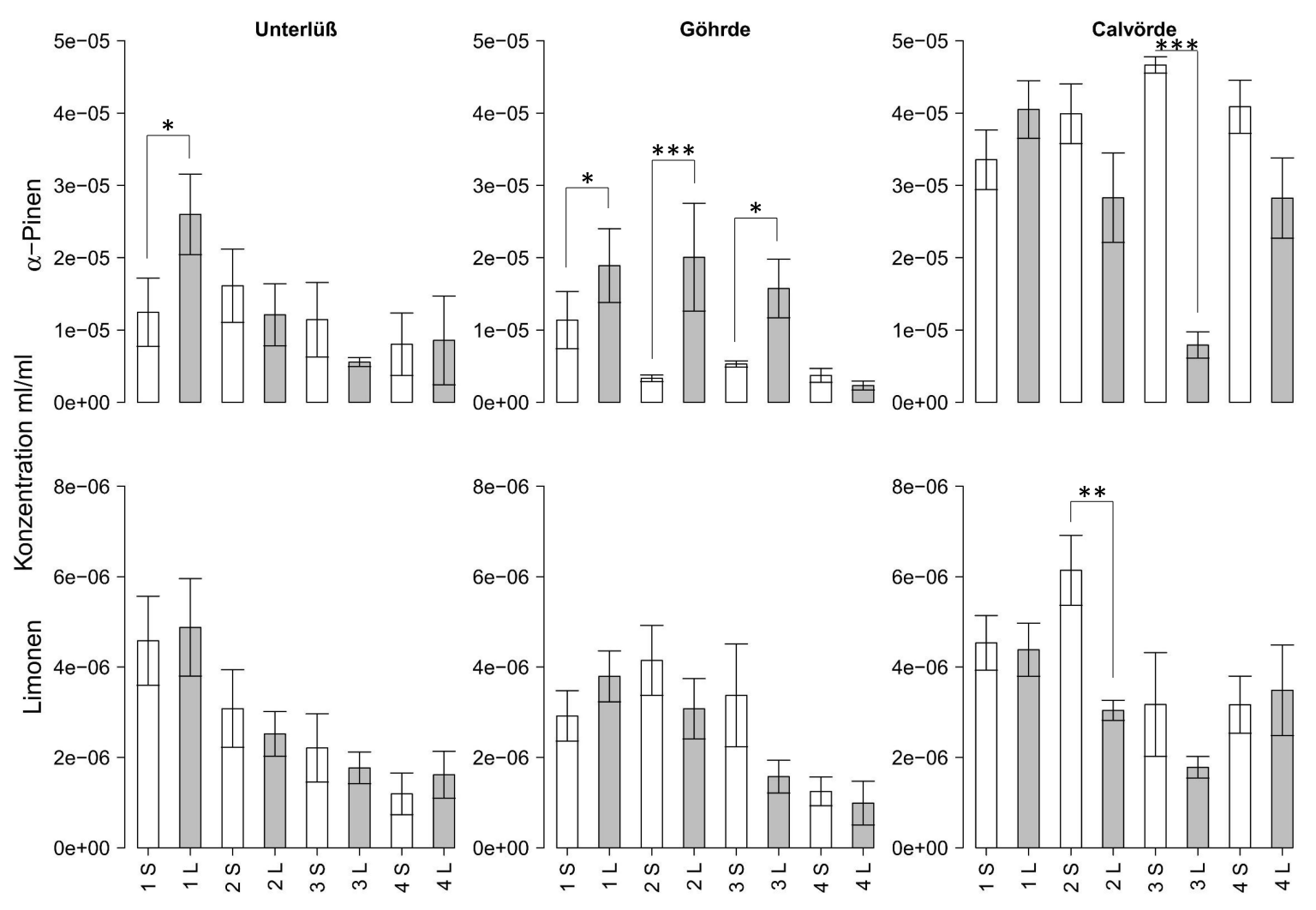

Abbildung 14: Emittierte Konzentrationen von $\alpha$-Pinen und Limonen innerhalb von zwei Stunden über einen Stamm von F. sylvatica zu unterschiedlichen Jahreszeiten und jährlichen Niederschlagsmengen $(\bar{x} \pm$ s. e.). Wilcoxon-Test: * $=\mathrm{p} \leq 0,05,{ }^{* *}=\mathrm{p} \leq 0,01, * * *=\mathrm{p} \leq 0,001 . \mathrm{S}=$ Sand, $\mathrm{L}=$ Lehm, $1=$ Frühjahr, $2=$ Sommer, $3=$ Herbst, $4=$ Winter

Tabelle 6: Jahreszeitliche Konzentrationsveränderungen von $\alpha$-Pinen und Limonen für den jeweiligen Bodentyp

\begin{tabular}{|l|c|c|c|c|c|}
\hline \multicolumn{7}{|c|}{$\alpha$-Pinen } \\
\hline Standort & Bodentyp & Frühling & Sommer & Herbst & Winter \\
\hline \multirow{2}{*}{ Unterlüß } & $\mathrm{S}$ & $\mathrm{a}$ & $\mathrm{a}$ & $\mathrm{a}$ & $\mathrm{a}$ \\
\cline { 2 - 6 } & $\mathrm{L}$ & $\mathrm{a}$ & $\mathrm{a}$ & $\mathrm{a}$ & $\mathrm{a}$ \\
\hline \multirow{2}{*}{ Göhrde } & $\mathrm{S}$ & $\mathrm{a}$ & $\mathrm{a}$ & $\mathrm{a}$ & $\mathrm{a}$ \\
\cline { 2 - 6 } & $\mathrm{L}$ & $\mathrm{a}$ & $\mathrm{a}$ & $\mathrm{a}$ & $\mathrm{a}$ \\
\hline \multirow{2}{*}{ Calvörde } & $\mathrm{S}$ & $\mathrm{a}$ & $\mathrm{a}$ & $\mathrm{a}$ & $\mathrm{a}$ \\
\cline { 2 - 6 } & $\mathrm{L}$ & $\mathrm{a}$ & $\mathrm{ab}$ & $\mathrm{b}$ & $\mathrm{ab}$ \\
\hline \hline
\end{tabular}

\begin{tabular}{|c|c|c|c|c|c|}
\hline \hline \multicolumn{9}{|c}{ Limonen } \\
\hline Standort & Bodentyp & Frühling & Sommer & Herbst & Winter \\
\hline \multirow{2}{*}{ Unterlüß } & $\mathrm{S}$ & $\mathrm{a}$ & $\mathrm{ab}$ & $\mathrm{ab}$ & $\mathrm{b}$ \\
\cline { 2 - 6 } & $\mathrm{L}$ & $\mathrm{a}$ & $\mathrm{a}$ & $\mathrm{a}$ & $\mathrm{a}$ \\
\hline \multirow{2}{*}{ Göhrde } & $\mathrm{S}$ & $\mathrm{a}$ & $\mathrm{a}$ & $\mathrm{a}$ & $\mathrm{a}$ \\
\cline { 2 - 6 } & $\mathrm{L}$ & $\mathrm{a}$ & $\mathrm{a}$ & $\mathrm{a}$ & $\mathrm{a}$ \\
\hline \multirow{2}{*}{ Calvörde } & $\mathrm{S}$ & $\mathrm{a}$ & $\mathrm{a}$ & $\mathrm{a}$ & $\mathrm{a}$ \\
\cline { 2 - 6 } & $\mathrm{L}$ & $\mathrm{a}$ & $\mathrm{ab}$ & $\mathrm{b}$ & $\mathrm{ab}$ \\
\hline
\end{tabular}

Unterschiedliche Buchstaben geben signifikante Unterschiede in den Konzentrationen zwischen den Jahreszeiten wieder (Bonferroni T-Test, $\mathrm{p} \leq 0,05$ ). $\mathrm{S}=$ Sand, $\mathrm{L}=$ Lehm 


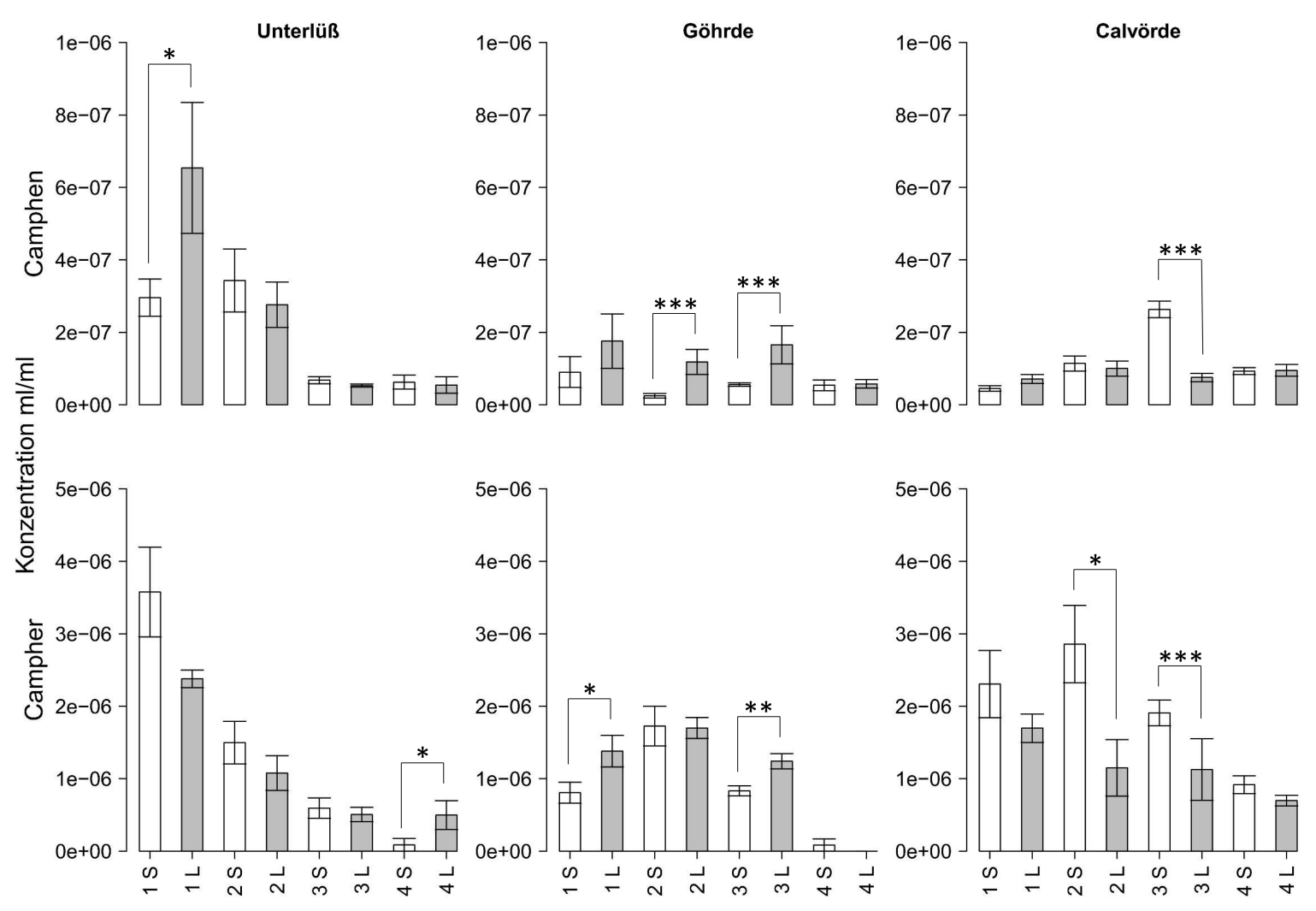

Abbildung 15: Emittierte Konzentrationen von Camphen und Campher innerhalb von zwei Stunden über einen Stamm von F. sylvatica zu unterschiedlichen Jahreszeiten und jährlichen Niederschlagsmengen $(\bar{x} \pm$ s. e.). Wilcoxon-Test: $*=\mathrm{p} \leq 0,05,{ }^{* *}=\mathrm{p} \leq 0,01,{ }^{* * *}=\mathrm{p} \leq 0,001 . \mathrm{S}=$ Sand, $\mathrm{L}=$ Lehm, $1=$ Frühjahr, $2=$ Sommer, $3=$ Herbst, $4=$ Winter

Tabelle 7: Jahreszeitliche Konzentrationsveränderungen von Camphen und Campher für den jeweiligen Bodentyp

\begin{tabular}{|l|c|c|c|c|c|}
\hline \multicolumn{7}{|c|}{ Camphen } \\
\hline Standort & Bodentyp & Frühling & Sommer & Herbst & Winter \\
\hline \multirow{2}{*}{ Unterlüß } & $\mathrm{S}$ & $\mathrm{ab}$ & $\mathrm{b}$ & $\mathrm{ac}$ & $\mathrm{c}$ \\
\cline { 2 - 7 } & $\mathrm{L}$ & $\mathrm{a}$ & $\mathrm{a}$ & $\mathrm{a}$ & $\mathrm{a}$ \\
\hline \multirow{2}{*}{ Göhrde } & $\mathrm{S}$ & $\mathrm{a}$ & $\mathrm{a}$ & $\mathrm{a}$ & $\mathrm{a}$ \\
\cline { 2 - 7 } & $\mathrm{L}$ & $\mathrm{a}$ & $\mathrm{a}$ & $\mathrm{a}$ & $\mathrm{a}$ \\
\hline \multirow{2}{*}{ Calvörde } & $\mathrm{S}$ & $\mathrm{a}$ & $\mathrm{b}$ & $\mathrm{c}$ & $\mathrm{ab}$ \\
\cline { 2 - 7 } & $\mathrm{L}$ & $\mathrm{a}$ & $\mathrm{a}$ & $\mathrm{a}$ & $\mathrm{a}$ \\
\hline \hline \multirow{2}{*}{ Standort } & Bodentyp & Frühling & Sommer & Herbst & Winter \\
\hline \multirow{2}{*}{ Unterlüß } & $\mathrm{S}$ & $\mathrm{a}$ & $\mathrm{b}$ & $\mathrm{b}$ & $\mathrm{b}$ \\
\cline { 2 - 7 } & $\mathrm{L}$ & $\mathrm{a}$ & $\mathrm{b}$ & $\mathrm{b}$ & $\mathrm{b}$ \\
\hline \multirow{2}{*}{ Göhrde } & $\mathrm{S}$ & $\mathrm{a}$ & $\mathrm{b}$ & $\mathrm{a}$ & $\mathrm{a}$ \\
\cline { 2 - 7 } & $\mathrm{L}$ & $\mathrm{a}$ & $\mathrm{a}$ & $\mathrm{a}$ & $\mathrm{b}$ \\
\hline \multirow{2}{*}{ Calvörde } & $\mathrm{S}$ & $\mathrm{a}$ & $\mathrm{a}$ & $\mathrm{a}$ & $\mathrm{a}$ \\
\cline { 2 - 7 } & $\mathrm{L}$ & $\mathrm{a}$ & $\mathrm{a}$ & $\mathrm{a}$ & $\mathrm{a}$ \\
\hline
\end{tabular}

Unterschiedliche Buchstaben geben signifikante Unterschiede in den Konzentrationen zwischen den Jahreszeiten wieder (Bonferroni T-Test, $\mathrm{p} \leq 0,05$ ). $\mathrm{S}=$ Sand, $\mathrm{L}=$ Lehm 


\subsubsection{Diskussion}

Das Stamm-VOC-Muster von F. sylvatica ist geprägt von vier Stoffgruppen (Terpene, Alkane, Aldehyde und Aromate), wobei Terpene die Hauptgruppe ausmachen. Dies deckt sich mit Untersuchungen zu den oberirdischen Pflanzenteilen von F. sylvatica aus anderen Studien. Demnach sind die Emissionen von Zweigen und Blätter von F. sylvatica ebenfalls durch MT geprägt 25, 40, 126, 143. Es ist bekannt, dass MT typische VOC sind, die von Pflanzen in die Atmosphäre emittiert werden [153. Zusätzlich zu den Terpenen enthalten pflanzliche Emissionen eine Anzahl weiterer VOC, unter anderem Alkohole, Ester, Ether, Aldehyde, Ketone, Alkene und Alkane [154], Stoffgruppen, die auch in dieser Studie im Stamm-VOC-Muster gefunden wurden.

Alle gefundenen Stoffklassen bis auf Alkane und Alkohole zeigen in ihrem Vorkommen eine Abhängigkeit zur Jahreszeit. Dies ist signifikant für die Stoffgruppe Terpene, die auf allen Untersuchungsflächen besonders in den Sommermonaten am häufigsten in den Proben vorkommt. Auch Holzke et al. (2006) konnten in ihrer VOC-Untersuchung von Blättern von F. sylvatica eine jahreszeitliche Abhängigkeit von Terpenen feststellen [126]. Demnach wurden die höchsten MT-Emissionen im Sommer gemessen und keine MT-Emissionen im Frühjahr, obwohl die Blätter in der Untersuchung von Holzke et al. (2006) schon vollständig entwickelt und die Temperatur- und Lichtbedingungen moderat waren [126]. Auch andere Laubbäume wie Malus domestica und Prunus avium zeigen eine Saisonalität bei den MT-Emissionen [155]. Rapparini et al. (2000) ordneten die saisonale Emissionsveränderungen den veränderten ökologischen Funktionen im Verlauf der Pflanzenentwicklung zu 155]. Diese Studie und die Untersuchung von Holzke et al. (2006) zeigen, dass die Jahreszeit einen nicht zu vernachlässigenden Einfluss auf die Emissionen von $F$. sylvatica besitzen.

Zusätzlich zu der jahreszeitlichen Zusammensetzung des VOC-Musters der Buchenstämme konnte bei zwei Stoffgruppen (Alkenen und Terpenen) eine Abhängigkeit zu der jährlichen Niederschlagsmenge festgestellt werden. Die Anzahl der in den Proben gefundenen Terpene nahm mit abnehmenden jährlichen Niederschlagsmengen auf den Standorten zu und bei den Alkenen konnten die höchste Anzahl in den Proben in der Mitte des Niederschlagsgradienten im Untersuchungsgebiet Göhrde bestimmt werden.

Der Bodentyp zeigte in dieser Studie keinen großen Einfluss auf das VOC-Muster. Nur für die Stoffgruppe Ester konnte ein signifikanter Effekt nachgewiesen werden. Es wurden in den Proben auf Sandböden mehr Ester gefunden als auf Lehmböden.

Anhand des dominanten Vorkommens der Terpene wurden aus dieser Stoffklasse vier MT (Camphen, Campher, $\alpha$-Pinen und Limonen) ausgewählt, um deren Konzentrationsverlauf in der Vegetationsperiode $2011 \mathrm{zu}$ bestimmen. Zusätzlich wurden für die Konzentrationsbestimmung zwei Aldehyde (Nonanal und Decanal) ausgewählt. 
$\alpha$-Pinen [14,28, 156, 157], Limonen [158], Camphen [157, 159] und Campher [159] sind bekannte Stress-VOC für Pflanzen und werden von diesen bei Trockenheit vermehrt abgegeben. Des Weiteren zählen $\alpha$-Pinen und Limonen zu den dominantesten MT, die von belaubten Zweigen von F. sylvatica emittiert werden [130,160]. Camphen gehört ebenfalls zu den MT, die im VOC-Muster von Rot-Buchenblättern vorkommen [40].

Alle vier MT zeigen im Frühjahr und Sommer die höchsten Konzentrationen. Eine mögliche Ursache könnten die höheren Temperaturen an den jeweiligen Messtagen darstellen, da die Terpen-Emissionen der Blätter von F. sylvatica temperatur-, aber auch lichtabhängig sind 126, 131, 132, 160|. Demnach werden höhere Terpen-Emissionen bei höheren Umgebungstemperaturen und höherer photosynthetisch aktiver Strahlung (PAR) gemessen. Eine Studie von Demarcke et al. (2010) wies höhere MT-Emissionen von Blättern von F. sylvatica im August und eine starke Abnahme dieser beginnend im späteren Verlauf des August über den September, welche sich bis zum Ende der Messung im Oktober fortsetzte, nach 133 .

Auffällig ist die signifikante Abhängigkeit der Konzentrationen von $\alpha$-Pinen und Limonen von den Niederschlagsmengen. Es werden über das ganze Jahr deutlich mehr $\alpha$-Pinen und Limonen in Calvörde, dem Standort mit den geringsten jährlichen Niederschlagsmengen, emittiert. Eine Untersuchung von der Abteilung Pflanzenökologie und Ökosystemforschung hat für das Untersuchungsgebiet Calvörde im Gegensatz zu Sellhorn und Göhrde einen gleichmäßig abnehmenden Grundflächenzuwachs seit 1970 festgestellt 104. Des Weiteren hat sich nicht nur die mittlere Jahresdurchschnittstemperatur in Calvörde im Gegensatz zu Sellhorn und Göhrde am stärksten erhöht (um 1,4 Grad), sondern auch die Niederschlagsmenge im Juni hat seit 1950 (von $76 \mathrm{~mm}$ auf $51 \mathrm{~mm}$ ) deutlich abgenommen 104. Dagegen ist die Niederschlagsmenge in Juni für Sellhorn, dem Untersuchungsgebiet mit dem höchsten jährlichen Niederschlagsmengen, von 1950 bis 2000 unverändert geblieben (78-79 mm). Dies spricht dafür, dass $\alpha$-Pinen und Limonen für Stämme von F. sylvatica als Stressmarker fungieren können.

Nonanal und Decanal sind bekannt dafür vermehrt bei Trockenstress emittiert zu werden [161. Sie zeigten in den Konzentrationen keine Unterschiede hinsichtlich der Niederschlagsmenge, dafür einen deutlichen jahreszeitlichen Trend. Insofern können die Aldehyde möglicherweise als Marker für kurzfristige Stressereignisse im Sommer und die Terpene $\alpha$-Pinen und Limonen als Stressmarker für ein längerfristig anhaltendes Stressereigniss an einem Standort dienen. Dies würde mit der Untersuchung von Kainulainen et al. (1991) übereinstimmen, wonach die Emissionen von $\alpha$-Pinen und Limonen und auch Camphen sich bei Picea abies nach anhaltendem Trockenstress erhöhten [157. Für $\alpha$-Pinen kann dies durch die Studie von Ormeño et al. (2007) ebenfalls bestätigt werden, wonach die Emissionen von $\alpha$-Pinen bei langanhaltender Trockenheit zunehmen [28]. 
Die Veränderungen von Terpen-Emissionen sind nicht nur von exogenen Faktoren, sondern auch von endogenen Faktoren wie dem Entwicklungsstand der Blätter abhängig [162 und können eine mögliche Erklärung für die hier gefundenen saisonalen Unterschiede in den Terpen-Emissionen sein. Der Entwicklungsstand der Blätter hat einen Einfluss auf die Photosynthese-Aktivität, welche eine regulierende Wirkung auf die Spaltöffnungen der Blätter besitzt und sich dadurch auf die Transpiration der Blätter und somit auch auf den Saftfluss im Stamm 163 und auf das VOC-Muster des Buchenstammes auswirken kann. Folglich zeigt diese Studie die Wichtigkeit von VOC-Messungen über eine gesamte Vegetationsperiode, um das Emissionsverhalten von Pflanzen wie F. sylvatica besser verstehen zu können.

Die Zusammenfassung der Monate zu Frühjahr, Sommer, Herbst und Winter entsprach den Entwicklungsstadien der Blätter (die sich im Austrieb befanden, voll entwickelt, verfärbt oder abgeworfen waren) von $F$. sylvatica auf den Untersuchungsgebieten im Untersuchungsjahr 2011. Die Blattentwicklung könnte man zukünftig auch mittels einer einheitlichen Skala mit leicht erkennbaren morphologischen Merkmalen, die für jeden nachvollziehbar sind, beschreiben. Im Nutzpflanzenanbau gibt es z. B. die BBCH-Skala von der Biologischen Bundesanstalt für Land- und Forstwirtschaft, dem Bundessortenamt und der chemischen Industrie für mono- und dikotyler Pflanzen, die gleiche phänologische Entwicklungsstadien mit einem zweistelligen dezimal Code belegen [164. In einer Untersuchung von Schuster et al. (2014) wurden bei der Beschreibung der Blattphänologie von F. sylvatica eine Adaption der BBCH-Skala für F. sylvatica nach Meier (1997) 165 und Finn et al. (2007) 166 verwendet 167. Weitere Studien, die sich mit der Blattphänologie von $F$. sylvatica beschäftigen, verwenden ebenfalls die BBCH-Skala, um zum Beispiel den Grad der Verfärbung einheitlich zu beschreiben [168, 169]. Vorteil der BBCH-Skala ist es, dass nicht nur die Hauptwachstumsphasen alleine berücksichtigt werden, sondern auch einzelne Zeitpunkte zum Beispiel während des Blattaustriebes. Ein weiterer Vorteil einer solchen Skala liegt in ihrer Vereinheitlichung und Vergleichbarkeit zwischen Wiederholungsuntersuchungen und verschiedenen Untersuchungen, da jeder einzelner Entwicklungsschritt mittels der BBCH-Skala genau definiert ist.

Für weitere Untersuchungen sollte bei jeder Messung der Entwicklungsstand der Blätter aufgenommen werden, um bei Messungen über mehrere Jahre auch eine mögliche saisonale Verschiebung der Entwicklung beim VOC-Muster besser berücksichtigen zu können. 


\subsection{Zusammenfassung}

Die in dieser Arbeit entwickelte Stammabsaugkammer ermöglicht ohne viel Aufwand das VOC-Muster von Baumstämmen zu bestimmen und hat sich inzwischen ebenfalls in der Abteilung Ökopedologie der Tropen und Subtropen der Fakultät für Forstwissenschaften und Waldökologie in Göttingen [170 sowie in der Forschungsgruppe Envirus (ENVIronment Under Stress) etabliert [171.

Für das VOC-Muster von F. sylvatica konnten insgesamt 116 verschiedene VOC identifiziert werden, die elf Stoffgruppen zugeordnet werden konnten. Weitere 76 Substanzen konnten nicht identifiziert werden und wurden als Gruppe „Unbekannt“ zusammengefasst. Dabei dominierten in den wärmeren Monaten vor allem VOC aus der Stoffgruppe Terpene und Aldehyde. Die Anzahl der unterschiedlichen VOC, aus denen sich das Stamm-VOCMuster zusammensetzt, war im Sommer am höchsten. Dies bestätigt die Hypothese, dass die Zusammensetzung des VOC-Musters von F. sylvatica sich im Jahresverlauf verändert.

Die Fragestellung, ob sich das VOC-Muster von Stämmen von F. sylvatica in Untersuchungsgebieten mit unterschiedlichen jährlichen Niederschlagsmengen verändert, konnte nicht eindeutig beantwortet werden. Zwar konnte nachgewiesen werden, dass das Vorkommen von vier Stoffklassen in den Proben signifikant von Niederschlagsmengen abhängt, darunter auch die Stoffgruppe der Terpene, die einen Großteil der vom Stamm von F. sylvatica emittierten VOC ausmachten, jedoch zeigten die restlichen Stoffgruppen keine Abhängigkeiten in ihrem Vorkommen in den Proben zu den Niederschlagsmengen. Da fast alle Stoffklassen, bis auf organische Säure, Acetat und Ether, deren Vorkommen in den Proben sehr gering war, in allen Untersuchungsgebieten vorkamen, konnte die Hypothese, dass sich die chemische Stoffklassenzusammensetzung der Stämme von F. sylvatica bei unterschiedlichen Niederschlagsmengen ändert, nicht bestätigt werden.

Trotz der gleichen chemischen Zusammensetzung konnten Unterschiede in der Emissionsrate von vier VOC ( $\alpha$-Pinen, Limonen, Nonanal und Decanal) nachgewiesen werden. Diese vier VOC sind als Stress-VOC in der Literatur bekannt. Somit konnten für die Stämme von F. sylvatica VOC identifiziert werden, die einen Hinweis auf den Stresszustand von $F$. sylvatica geben können. Alle vier VOC sind dafür bekannt zu dem VOC-Muster von trocken-gestressten Blättern von F. sylvatica zu gehören. Die dritte und letzte Hypothese, dass es mindestens ein VOC gibt, dass als Anzeiger-VOC für trocken-gestresste Bestände von F. sylvatica dienen kann, konnte bestätigt werden. 


\section{Wurzel-Volatilenmuster von Rot-Buche ( $F$. sylvatica)}

Christine Rachow

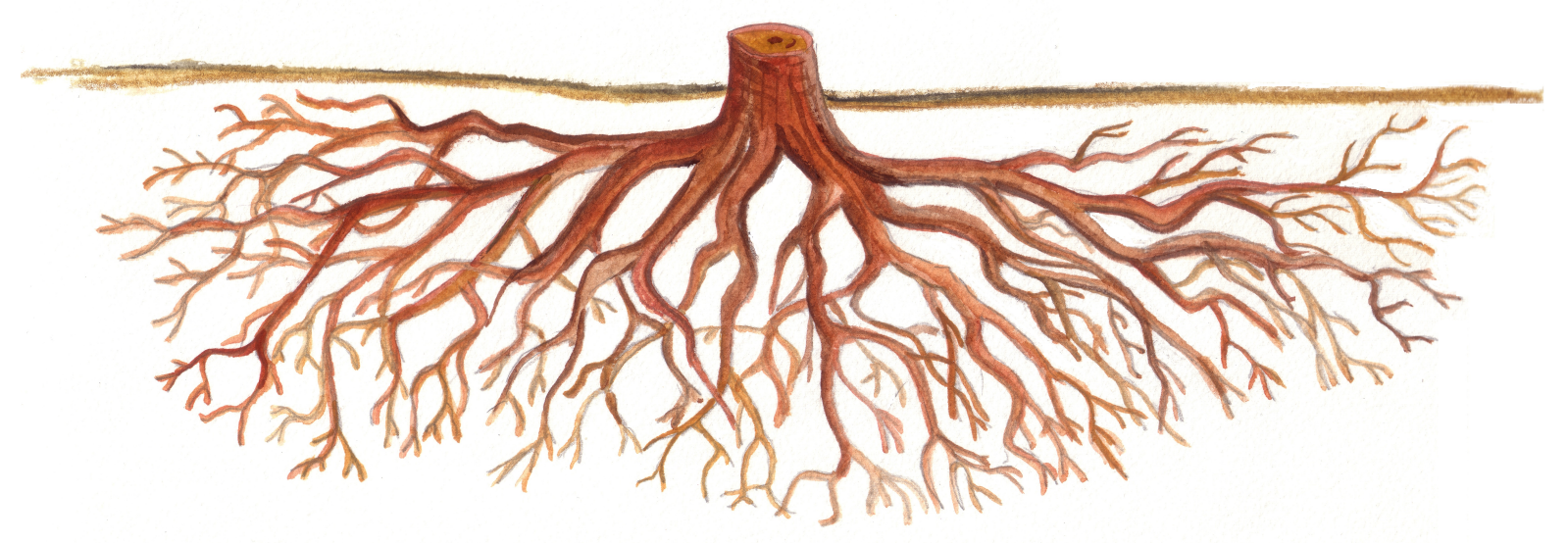

Abbildung 16: Schematische Darstellung eines Wurzelsystems

Teile des Inhaltes dieses Kapitels wurden in „Mitteilungen der Deutschen Gesellschaft für allgemeine und angewandte Entomologie“ modifiziert veröffentlicht, Band 21 (2018): 313-318, ISSN: 0344-9084 


\section{Wurzel-Volatilenmuster von Rot-Buche ( $F$. sylvatica)}

Studien zeigen, dass Buchenwälder auf die mit dem Klimawandel einhergehenden steigenden Temperaturen und die damit verbundene Zunahme von Trockenperioden empfindlich reagieren [9]. Diese Auswirkungen können nicht nur die Massenvermehrung von verschiedenen Borkenkäferarten [8, 97, 98, 126], sondern auch die Vermehrung von wurzelfressenden Insektenstadien, beispielsweise dem Engerling des Waldmaikäfers (Melolontha hippocastani), unterstützen 172, 173. Daher ist es nicht nur wichtig zu wissen, wie die oberirdischen Pflanzenorgane auf Trockenheit reagieren, sondern auch, welche Auswirkung der Klimawandel auf die Wurzelorgane der Bäume hat. Das VOC-Muster der Wurzeln kann einen Aufschluss über den Zustand der Bäume liefern, bevor der Schwächezustand von F. sylvatica anhand von frühzeitigem Laubabwurf oberirdisch sichtbar wird. Stressinduzierte Wurzel-VOC können somit bei der Stressfrüherkennung in Buchenbeständen und bei der Zustandseinschätzung von Beständen eine wichtige Rolle spielen.

Besonders Feinwurzeln (d. h. Wurzeln $<2$ mm Durchmesser), die den aktivsten Teil der Wurzel für die Nährstoff- und Wasseraufnahme darstellen, reagieren auf Trockenheit sehr sensibel [79]. An den Feinwurzeln selber kommt eine Vielzahl von Mikroorganismen vor, die von den Wurzel-Exsudaten und den VOC profitieren und die die Wurzel selbst durch ihr VOC-Muster sowohl positiv als auch negativ beeinflussen können 174, 175]. Die chemischen Interaktionen zwischen Wurzeln und anderen im Boden lebenden Organismen sind intensiv. Wurzel-Exsudate haben einen großen nährenden und signalisierenden Effekt auf die Boden-Mikroorganismen und -Mesofauna 176, 177 und das Wurzel-VOC-Muster kann das Verhalten der Makrofauna im Boden beeinflussen [42. Diese Interaktionen zwischen Wurzeln und Bodenorganismen beeinflussen die Reaktionen von Bäumen auch im Hinblick auf zu erwartende Stressereignisse im Klimawandel. Das steigende Interesse an Wurzel-VOC unterstreicht den Forschungsbedarf an deren Bedeutung bei der unterirdischen Kommunikation 178,179.

Um die Interaktionen an Feinwurzeln besser zu verstehen, befasst sich diese Studie im Kapitel 4.2 mit dem VOC-Muster von Feinwurzeln von F. sylvatica auf sandigem Boden entlang eines Niederschlagsgradienten. Im Kapitel 4.3 wird die Entwicklung einer Apparatur zur Messung der horizontalen Bodenbeweglichkeit von Wurzel-VOC beschrieben. Das Wissen über die Ausbreitung von Wurzel-VOC im Boden kann Aufschluss darüber geben, ob und über welche Distanzen sich bodenlebende und wurzelfressende Insektenstadien mittels VOC ihre Nahrung aufsuchen können. 
In diesem Kapitel werden, nach einem theoretischen Hintergrund mit dem bisherigen Forschungsstand zum VOC-Muster des Waldbodens (s. Kapitel 4.1), folgende vier Forschungsfragen mit zugehörigen Hypothesen untersucht:

Forschungsfrage 1: Welche VOC werden von F. sylvatica über ihre Feinwurzeln abgegeben und welche VOC und Stoffgruppen dominieren dabei das VOC-Muster?

Hypothese 1: Wurzeln von F. sylvatica emittieren eine Vielzahl von VOC, die sowohl buchentypisch sind als auch auf die Anwesenheit von Pilzen und Bakterien an der Feinwurzel hindeuten können.

Forschungsfrage 2: Welchen Einfluss hat der Niederschlagsgradient auf die Emissionen von Wurzel-VOC auf sandigen Böden?

Hypothese 2 a: Die Emission von Wurzeln-VOC von F. sylvatica unterscheidet sich zwischen Standorten mit geringerem jährlichen Niederschlag und denen mit höherem jährlichen Niederschlag.

Hypothese 2 b: Es gibt mindestens ein VOC, dass als Marker-VOC zur Erkennung von Wurzel, die weniger gut wasserversorgt sind, verwendet werden kann.

Forschungsfrage 3: Verändert sich das Wurzel-VOC-Muster saisonal? Gibt es Unterschiede im Wurzel-VOC-Muster zwischen den Jahreszeiten Herbst und Frühling?

Hypothese 3: Die Wurzel-VOC-Muster von Herbst und Frühling unterscheiden sich in ihrer VOC-Zusammensetzung. Im Frühjahr werden zu Beginn der Vegetationsperiode mehr VOC emittiert als im Herbst.

Forschungsfrage 4: Ist es möglich, die Bodenbeweglichkeit von Wurzel-VOC zu messen?

Hypothese 4: Wurzel-VOC können sich mittels Diffusion durch den Boden bewegen und besitzen dadurch eine bestimmte Reichweite für mögliche Interaktionen mit weiteren Akteuren im Boden. 


\subsection{Theoretischer Hintergrund}

\subsubsection{Wurzelsystem von $F$. sylvatica}

F. sylvatica besitzt eine Herzwurzel [73], welche auf trockenen Böden mehrere Meter tief in den Boden dringen kann, um an Wasser zu gelangen, und welche die Verankerung im Boden gewährleistet. Die kurzen Seitenwurzeln mit einer Vielzahl von kurzen und extrem dünnen Feinwurzeln ermöglichen F. sylvatica ein relativ kleines Bodenvolumen sehr effektiv auszunutzen [75]. Die Feinwurzeloberfläche eines alten Buchenwaldes ist zwischen 50 und $100 \%$ größer als die Blattoberfläche des Bestandes [180]. Daraus ergibt sich eine große unterirdische Oberfläche für die VOC-Emissionen.

Die höchsten Wurzeldichten wurden in Buchenbeständen in den oberen Bodenhorizonten gefunden; der Hauptteil der Feinwurzelmasse reicht bis in $40 \mathrm{~cm}$ Tiefe [180]. Der Anteil der Biomasse, die die lebenden Feinwurzeln in der organischen Auflage und im Mineralboden ausmachen, variiert dabei abhängig von Bodentyp, Humusform und vom geologischen Substrat 180.

Die Feinwurzeln von F. sylvatia leben häufig in Symbiose mit Mykorrhizapilzen [181]. Die Pilzhyphen stehen in direktem Kontakt mit der Pflanze. Man unterscheidet dabei zwischen Ektomykorrhiza, bei denen das Mycel einen dichten Mantel um die Wurzelenden bildet (z. B. Arten der Gattung Tuber und Paxillus), und Endomykorrhiza, bei denen die Hyphen des Pilzes in die Zellen der Wurzelrinde eindringen 181 (Abb. 17). Die Mykorrhizierung sorgt für einen Schutz vor Wurzelpathogenen (z. B. sind Arten der Gattung Trichoderma Antagonisten zu phytopathogenen Pilzen) [182 und erhöht die Trockenresistenz der Pflanze durch die Vergrößerung der wasseraufnehmenden Oberfläche [183. F. sylvatica geht vorwiegend mit Ektomykorrhizapilzen Symbiosen ein 184. Pilze, die als Ektomykorrhiza mit F. sylvatica leben, sind unter anderem der Maronenröhrling (Imleria badia), der Rötliche Lacktrichterling (Laccaria laccata) sowie die an F. sylvatica häufig vorkommenden Pilze wie Rotfußröhrling (Xerocomellus chrysenteron), Scharfer Milchling (Lactarius pyrogalus) und Rotgegürtelter Milchling (Lactarius rubrocinctus) 185].

Mehr als $95 \%$ der Feinwurzeln von den meisten terrestrischen Pflanzen sind mit Mykorrhizapilzen kolonisiert, welche wiederum umgeben sind von komplexen mikrobiellen Gemeinschaften, die sich aus Mykorrhizahelferbakterien zusammensetzen [186, 187]. 

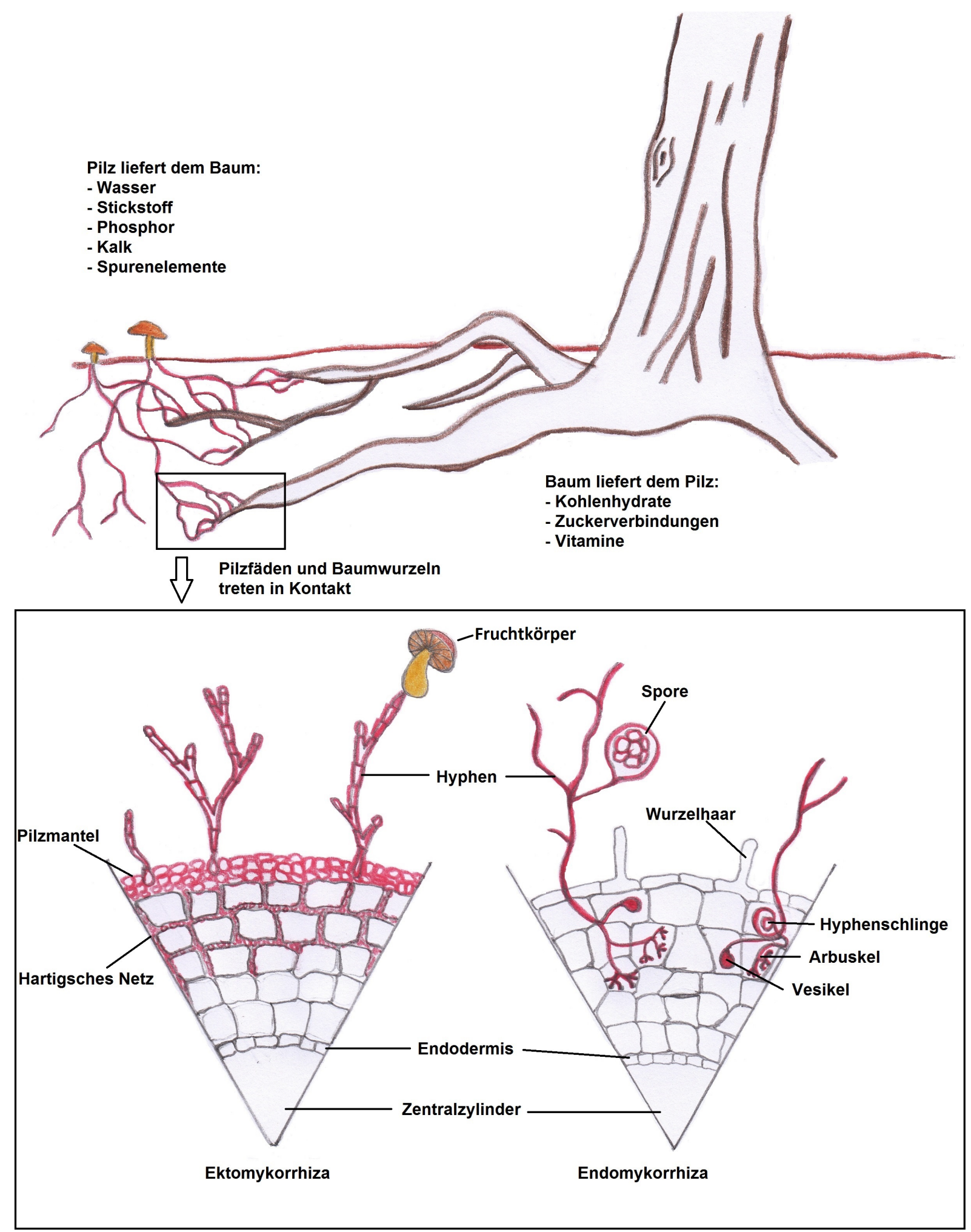

Abbildung 17: Schema der unterschiedlichen Mykorrhizatypen in Verbindung mit Baumwurzeln: Ekto- (links) und Endomykorrhiza (rechts) (verändert nach [1]) 


\subsubsection{VOC im Waldboden}

Der Waldboden wurde eine lange Zeit als VOC-Quelle unterschätzt. Inzwischen ist bekannt, dass Mirkroorganismen und Pflanzenwurzeln im Boden eine der Hauptquellen für VOC sind [188]. Die meisten bisherigen Untersuchungen beziehen sich auf Boden-VOC [189], die Rhizosphäre als Quelle für VOC wurde bisher kaum berücksichtigt 190,191]. Die Rhizosphäre bezeichnet eine wenige Millimeter breite Zone um die Wurzel herum [192]. Sie wird direkt durch die Wurzel beeinflusst [193] und ist der Bereich, wo Pflanzenwurzel und Bodenorganismen miteinander interagieren (Abb. 18). Somit ist sie eines der komplexesten Ökosysteme der Erde [194]. Pflanzenwurzeln geben eine Vielzahl an löslichen und unlöslichen oder flüchtigen Stoffwechselprodukten in die Rhizosphäre ab, wodurch die Rhizosphäre zu einer Nährstoffressource im Boden wird, welche verschiedene Populationen von Mikrobiota, inklusive Bakterien und Pilze, anlockt 195 197]. Diese verschiedenen Organismen konkurrieren um dieselben Ressourcen und zeigen verschiedene interspezifische Interaktionen. Ebenso wie die Wurzel geben die Mikroorganismen VOC in die Rhizosphäre ab. Die Komplexität der VOC-Muster, die in der Rhizosphäre beobachtet werden kann, legt nahe, dass die oberirdischen VOC nicht alleine eine wichtige Rolle in der interspezifischen Interaktion spielen.

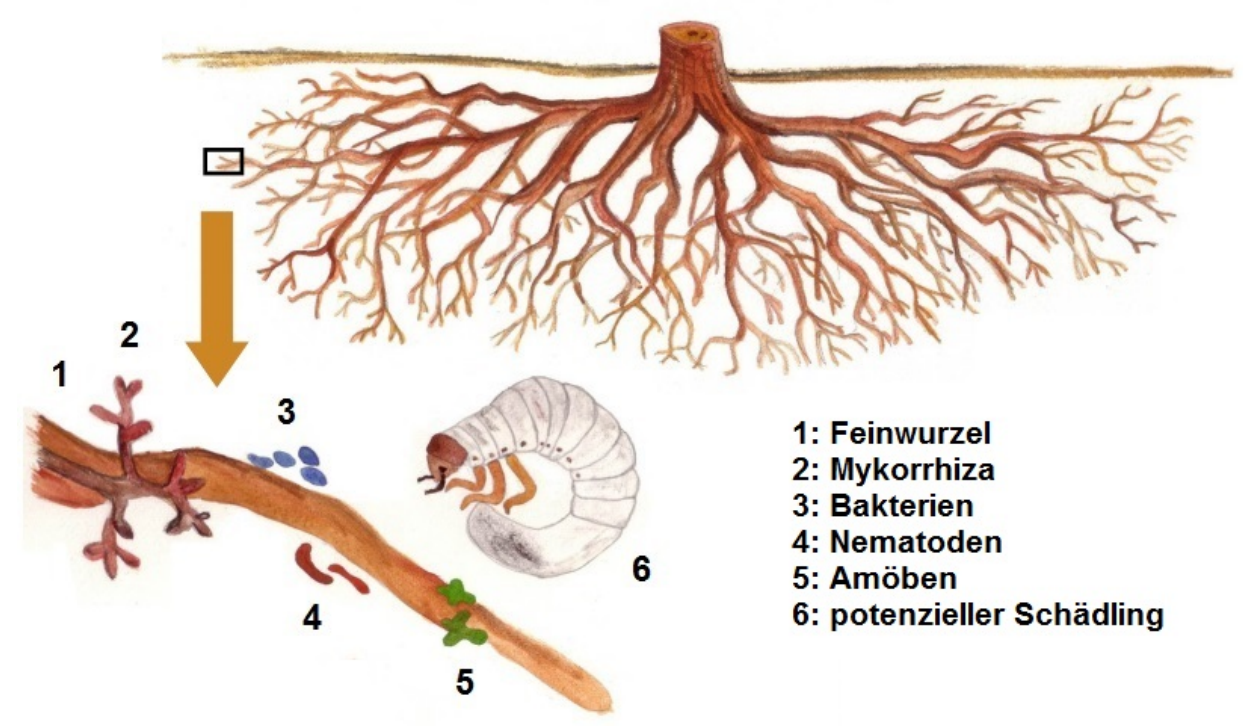

Abbildung 18: Beispiel für Mikro- und Makrofauna in der Rhizosphäre

Viele der Interaktionen von Organismen beruhen dabei auf VOC-Freisetzung durch die Pflanzenwurzel in die Rhizosphäre [198]. Durch die unterirdische Freisetzung von VOC zur Selbstverteidigung gegen Herbivore und pflanzenpathogene Pilze und Bakterien, zur Unterstützung von Symbiosen und zur Bekämpfung konkurrierender Arten können Pflanzen 
direkt oder indirekt Einfluss auf die Zusammensetzung der im Erdboden lebenden Organismen nehmen [199]. Durch die unterirdische Nutzung von VOC können Pflanzen die Anwesenheit von Nachbarpflanzen erkennen, ihr Wachstum anpassen und das Wachstum von anderen Wurzeln beeinflussen [179,200].

Bodenorganismen haben zudem einen Einfluss auf das VOC-Muster von Pflanzenwurzeln. Das VOC-Muster von Pflanzenwurzeln, die eine Symbiose mit arbuskulären Mykorrhizapilzen (AM) eingehen, verändert sich zu dem der nicht mykorrhizierten Wurzeln. Sun und Tang (2013) fanden in ihrer Untersuchung heraus, dass sich sowohl die Quantität als auch die Zusammensetzung des VOC-Musters bei Pflanzen mit AM stark verändert hat. Pflanzen mit AM emittieren demnach mehr Alkohole, Alkene, Ether und Säuren, aber weniger unverzweigte Alkene 201. Auch andere symbiotische Pilze können eine Veränderung der Wurzel-VOC-Muster verursachen und dadurch das Verhalten von im Erdboden lebenden Insekten beeinflussen 202].

Neben Mikroorganismen nutzen herbivore Insekten VOC im Boden zur Orientierung. Mittels VOC sind herbivore Insekten im Waldboden fähig ihre Wirtspflanzen über weite Distanzen hinweg aufzufinden und anhand des VOC-Musters die Qualität der Wirtspflanze abzuschätzen [202]. Qualitative und quantitative Veränderungen im Wurzel-VOCMuster können durch herbivore und mikrobielle Aktivitäten oder durch abiotischen Stress verursacht werden 202]. Somit können Veränderungen im VOC-Muster Indikatoren für Konkurrenz, für induzierte Verteidigung oder geringe Nahrungsqualität sein 203 205]. Im Gegensatz zu den oberirdischen Herbivoren gibt es zu den Mechanismen der Wirtsauffindung von bodenlebenden Insekten und zur Rolle, die Wurzel-VOC und $\mathrm{CO}_{2}$-Emissionen dabei spielen, wenige Untersuchungen 206,207.

Das VOC-Muster von Wurzeln von F. sylvatica ist kaum untersucht und das Wissen über die Interaktionen zwischen den Wurzel-VOC von F. sylvatica und den Bodenorganismen ist weitestgehend unbekannt. Es ist bereits dokumentiert, dass die von Wurzeln in die Rhizosphäre emittierten VOC eine wichtige Rolle im Ökosystem Boden spielen 188, 198. Das Wissen, welche Rolle diese VOC bei der Interaktion zwischen Pflanzen selbst spielen, ist dagegen weniger gut erforscht [179]. Nachfolgend soll anhand der Stoffgruppen Terpene und Aldehyde (Abb. 19 und 20) aufgezeigt werden, wie die verschiedensten Organismengruppen mittels VOC das Zusammenspiel in der Rhizosphäre beeinflussen können. Beide Stoffgruppen gehören mit zu den zehn Hauptstoffgruppen, die Bakterien und Pilze emittieren 188.

Terpene gehören zu den am häufigsten gemessenen aus dem Boden stammenden VOC in Feldstudien 190, 208, 209. Im Boden werden Terpene über Wurzeln abgegeben, die somit eine gute Terpenquelle darstellen [188, 190, 210 212. Durch die Freisetzung von Terpenen verbessern die Wurzeln den biologischen Abbau von xenobiotischer Verschmut- 
zung [213] und besitzen einen indirekten allelopathischen Effekt durch die Verminderung von Nitrifikation im Waldboden (Abb. 19. (1)) [214]. Von Wurzeln freigesetzte Terpene können negativ auf wurzelpathogene Pilze (z. B. Fomes annosus) wirken (Abb. 19 , (2)) 215, das vegetative Wachstum von Mykorrhizapilzen hemmen (Abb. 19, (2)) 216] oder Mykorrhizapilze zur Verzweigung ihrer Hyphen veranlassen [217].

Im Gegenzug können auch die Mykorrhizapilze durch die Abgabe von ST die Wurzelausbreitung verringern und einen Einfluss auf die Wurzelstruktur nehmen (Abb. 19, (3)) 174. Des Weiteren zeigen mykorrhizierte Wurzeln eine starke Stimulation von Genen, die bei der Synthese von MT involviert sind 218].

Wichtige Bodenorganismen wie Pseudomonas fluorescens, ein Mykorrhizahelferbakterium, welches das Wachstum von pflanzenpathogenen Keimen verhindert, nutzen das von den Wurzeln in der Rhizosphäre freigesetzte Terpen $\alpha$-Pinen als Kohlenstoff- und Energiequelle (Abb. 19, (2)) 211,219]. Auch die Wurzeln können durch die Terpenfreisetzung von Bakterien profitieren, indem die Bakterien Terpene abgeben, die das Mycelwachstum von pathogenen Pilzen hemmen können (Abb. 19. (4)) [175].

Bei Vorkommen von pathogenen Bakterien und Pilzen können Pflanzen Terpene mit antimikrobischen Eigenschaften, z. B. 1,8-Cineol, freisetzen (Abb. 19, (2;5)) 220,221. Über die Abgabe von Terpenen können die Wurzeln das Vorkommen von Nematoden im Boden beeinflussen. Bei einem Befall mit einem Schadinsekt können Pflanzenwurzeln nützliche Nematoden durch die Freisetzung von Terpenen anlocken (Abb. 19, (6)) 222. Allerdings können auch entomopathogene Nematoden durch die Freisetzung des Terpens $\beta$-Caryophyllen angelockt werden (Abb. 19, (6)) 223. In diesem Fall können Pflanzenwurzeln z. B. Limonen [211, ein Terpen mit nematizider Wirkung, freisetzen (Abb. 19 , (6)) [198].

Von den Wurzeln freigesetzte MT können nicht nur für die Wurzeln nützliche Organismen herbeirufen, sondern auch herbivore Insektenstadien wie den Engerling des Waldmaikäfers (M. hippocastani) und die Larven des westlichen Maiswurzelbohrers (Diabrotica virgifera) anlocken (Abb. 19, (7)) 42, 224,225. In dem Fall können Wurzeln Terpene abgeben, die repellent auf Insekten wirken können, z. B. Limonen, oder die eine Verteidigungsfunktion gegenüber Insekten besitzen (Abb. 19, (7)) 226, 227]. 


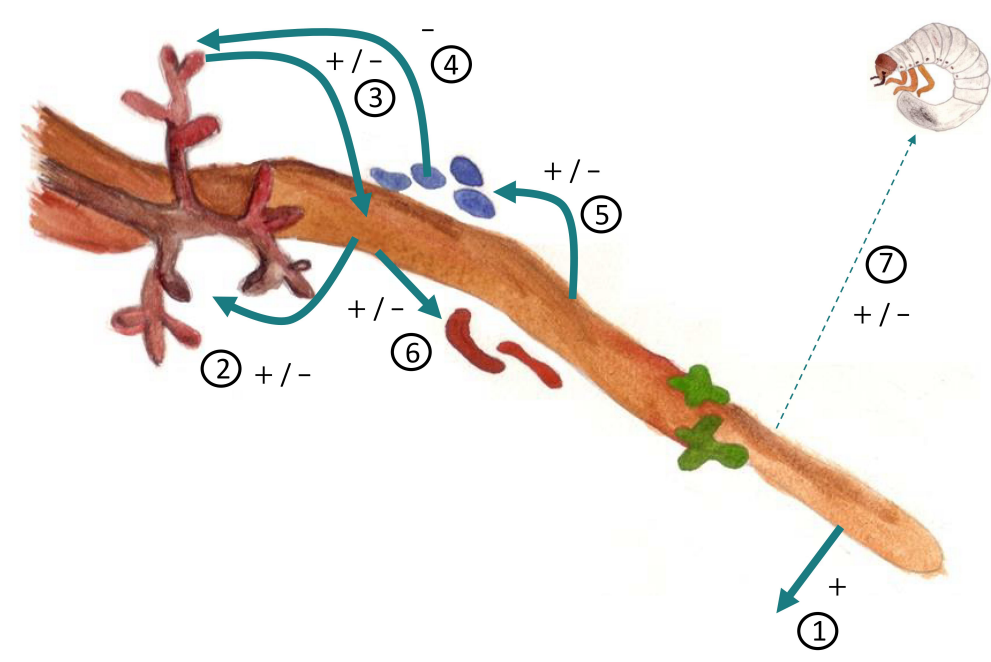

Abbildung 19: Bedeutung von Terpenen bei der Interaktion von unterschiedlichen Organismengruppen in der Rhizosphäre. + = positive Auswirkung; - = negative Auswirkung

Aldehyde, welche von den Wurzeln abgeben werden, können wie die Terpene eine nematizide (Abb. 20, (1)) [198, aber auch eine antimykotische Wirkung haben (Abb. 20. (2) 220,228 . Bodenbakterien können nicht nur mittels Terpenen das Mycelwachstum von pathogenen Pilzen hemmen, sondern auch durch die Freisetzung von Aldehyden wie Decanal und Nonanal (Abb. 20, (3)) [229]. Dies gilt auch für von Pilzen freigesetzte Aldehyde. So können Pilze mittels Benzaldehyd das Wachstum anderer Pilze hemmen (Abb. 20, (4)) [230]. Von der Wurzel abgegebene Aldehyde können zudem eine repellente Wirkung auf Insektenstadien besitzen (Abb. 20, (5)) 231.

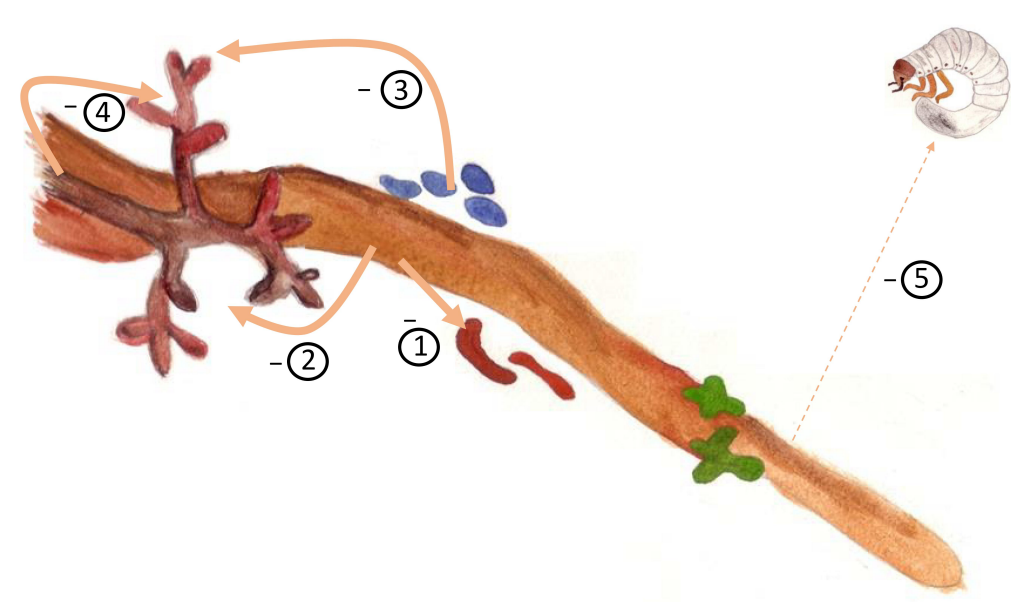

Abbildung 20: Bedeutung von Aldehyden bei der Interaktion von unterschiedlichen Organismengruppen in der Rhizosphäre. $+=$ positive Auswirkung; $-=$ negative Auswirkung 


\subsubsection{Bisherige Methoden zur Messung von Wurzel-VOC}

Obwohl schon 1974 das Wurzel-VOC-Muster untersucht wurde [232], wird bis heute vorwiegend das VOC-Muster der oberirdischen Pflanzenorgane statt der Pflanzenwurzeln bestimmt. Dementsprechend ist es nicht verwunderlich, dass es noch keine einheitliche Methode zur Messung von Wurzel-VOC gibt.

Viele klassische Methoden nutzen für ihre Messungen Wurzelextrakte von gewaschenen, klein geschnittenen und teilweisen abgestorbenen Wurzeln, wodurch es zu einer Verzerrung des natürlichen Wurzel-VOC-Musters kommen kann [226,232 234]. Hinzu kommt, dass die bisherigen Untersuchungen von Wurzel-VOC unter Labor- oder Klimakammerbedingungen durchgeführt wurden. Außerdem wurden bei den Untersuchungen von Baumwurzeln junge Setzlinge verwendet 211,235] und nicht Wurzeln erwachsener Bäume wie in dieser Studie. Untersuchungsergebnisse von Wurzel-VOC unter Laborbedingungen können unter Umständen ein verfälschtes Bild von den Wurzel-VOC-Emissionen unter natürlichen Bedingungen im Freiland erzeugen. Asensio et al. (2007) nehmen dies ebenfalls für bisherige Boden-VOC-Untersuchungen an 208]. Bis jetzt gibt es noch keine Studie, die den Einfluss von abiotischen Parametern auf das VOC-Muster von Wurzeln unter Freilandbedingungen untersucht hat [188]. Des Weiteren wurden bisherige Messungen von Wurzel-VOC größtenteils mit krautigen Pflanzen durchgeführt. Untersuchung zu WurzelVOC-Mustern von Bäumen gibt es bisher zu Pinus pinea [211], Qeurcus sp. 42, 224] und Malus domestica 235].

\subsubsection{Untersuchungsgebiete}

Die Untersuchung der Feinwurzel-VOC-Muster von F. sylvatica wurde in drei Buchenaltbeständen in drei Untersuchungsgebieten (Sellhorn, Unterlüß und Klötze) durchgeführt. Alle Untersuchungsgebiete befanden sich auf sauren und nährstoffarmen sandigen Böden (s. Kapitel 2.5. Tab. 1) und unterschieden sich in der Niederschlagsmenge. Der durchschnittliche jährliche Niederschlag nahm mit zunehmender Kontinentalität vom östlichen Niedersachsen zum westlichen Sachsen-Anhalt ab (816-614 mm). Die durchschnittliche Jahrestemperatur unterschied sich dagegen nur wenig innerhalb der Untersuchungsgebiete und stieg von $8,4{ }^{\circ} \mathrm{C}$ (Sellhorn und Unterlüß) leicht auf $8,7{ }^{\circ} \mathrm{C}$ (Klötze) an [2]. Die sandigen Versuchsflächen enthielten durchschnittlich $16 \%$ feinkörniges Bodenmaterial in den oberen $30 \mathrm{~cm}$ des Mineralbodens 236]. Die nutzbare Wasserspeicherkapazität (nWSK) variierte zwischen 79 (Unterlüß) und $111 \mathrm{~mm}$ (Sellhorn) in einer Bodentiefe von 1,2 m (Tab. 1). 


\subsection{Wurzel-VOC-Messung}

Bisher wurde die Reaktion von oberirdischen Pflanzenorganen auf Trockenheit mehr Beachtung zuteil als den unterirdischen Organen von Bäumen [79]. Das VOC-Muster von oberirdischen Pflanzenorganen von F. sylvatica wurde bereits in mehreren Studien untersucht 39, 126, 127] (s. Kapitel 3.1.2). Um ein besseres Verständnis von den ökologischen Interaktionen in einem Buchenwald zu erhalten, ist es notwendig, sowohl die oberirdischen als auch die unterirdischen VOC-Muster zu entschlüsseln.

In der Literatur gibt es gegensätzliche Hinweise, wie Feinwurzeln von F. sylvatica auf Trockenheit reagieren. Hertel et al. 236] beobachteten eine zunehmende Feinwurzelbiomasse mit abnehmendem Niederschlag. Andere Studien berichten von keinen Veränderungen in der Feinwurzelbiomasse 237] oder einer Abnahme in der Feinwurzelbiomasse $79,81,238$ bei abnehmendem Niederschlag.

Zusätzlich zur Trockenheit beeinflusst die Jahreszeit das Wurzelwachstum. Die Rate des Wurzelwachstums ist im Frühjahr am höchsten und im Herbst am geringsten [9,239.

In diesem Kapitel wird daher untersucht, wie sich das Feinwurzel-VOC-Muster von F. sylvatica in drei gleich alten Buchenbeständen auf sandigem Boden entlang eines Niederschlagsgradienten verändert und ob sich das VOC-Muster der Wurzeln saisonal zwischen Herbst und Frühjahr unterscheidet.

Die Freilegung der Wurzeln im Herbst erfolgte in Zusammenarbeit mit der Abteilung Pflanzenökologie und Ökosystemforschung der Georg-August-Universität.

\subsubsection{Material und Methoden}

\section{Probenahme}

Im Herbst 2013 und im Frühjahr 2015 wurde das VOC-Muster von Feinwurzeln in den Buchenbeständen der Untersuchungsgebiete Sellhorn, Unterlüß und Klötze untersucht. Alle VOC-Proben der Feinwurzeln mit den sich daran befindlichen Mikroorganismen einer Versuchsfläche wurden innerhalb eines Tages genommen. Die Probenahme im Herbst unterschied sich dabei leicht von der Probenahme im Frühjahr.

Im Herbst wurden auf jeder Versuchsfläche die Wurzeln samt Feinwurzeln durch das Ausheben von drei Löchern (3 m lang, $1 \mathrm{~m}$ breit und 2,5 m tief) freigelegt. Die Bodenlöcher wurden so auf der Fläche verteilt, dass der nächste Stamm mindestens $3 \mathrm{~m}$ von dem jeweiligen Loch entfernt stand. Dadurch sollte vermieden werden, dass die Hauptwurzeln der Bäume durch das Ausheben der Löcher beschädigt werden. Der Abstand zwischen den jeweiligen Bodenlöchern war im Mittel 15 m. In jedem Bodenloch wurden jeweils zwei VOC-Proben von den freigelegten Feinwurzeln aus dem B-Horizont und eine VOC-Probe aus dem A-Horizont genommen. Daraus ergaben sich neun VOC-Proben je Versuchsfläche. 
Die Lufttemperatur während der Herbstmessungen betrug $10-12,5{ }^{\circ} \mathrm{C}$ und die relative Luftfeuchtigkeit lag zwischen 60-85\%.

Im Frühjahr 2015 wurden ebenfalls in denselben Untersuchungsgebieten jeweils neun VOC-Proben von freigelegten Feinwurzeln genommen. In jedem Gebiet wurden zuerst neun Bäume mit ähnlichem Alter und BHD ausgewählt. Aufgrund dessen, dass im Herbst keine Unterschiede in den VOC-Messungen aus dem A- und B-Horizont gemessen wurden und dass die Arbeitsgruppe Pflanzenökologie und Ökosystemsforschung über $2 / 3$ der gesamten Feinwurzelbiomasse in der organischen Auflage und im oberen Mineralboden bis $30 \mathrm{~cm}$ Tiefe in den Untersuchungsgebieten nachweisen konnte [105], wurden im Frühahr anstelle von tiefen Bodenlöcher nur Feinwurzeln aus dem A-Horizont bis zu einer Tiefe von $10 \mathrm{~cm}$ beprobt. Dafür wurde im Abstand von 1,7 bis $3 \mathrm{~m}$ zum Buchenstamm mit einer Handschaufel die Streu vom Boden entfernt und der freigelegte A-Horizont vorsichtig aufgelockert, bis eine einzelne Feinwurzel freigelegt werden konnte. Je Baum wurde eine VOC-Probe der Feinwurzel genommen. Die Lufttemperatur im Frühjahr betrug während der Messungen $11,3-25,3{ }^{\circ} \mathrm{C}$ und die relative Luftfeuchtigkeit lag zwischen 45,3 und 88,7 \% (Anhang Tab. A.7).

In allen Feinwurzel-Proben kamen mykorrhizierte Wurzelspitzen vor (Anhang Abb.A.1) und die Länge der jeweils gemessenen Feinwurzel wurde notiert (Anhang Tab. A.8 und Tab. A.9. Im Frühjahr wurden zudem der BHD und der Abstand der beprobten Feinwurzel zu dem zugehörigen Stamm von F. sylvatica mit notiert (Anhang Tab. A.9.).

\section{Messsystem}

Die Beprobung erfolgte nach dem Grob Close-Loop-Verfahren [240|. Für die Methode zur Beprobung der Feinwurzeln wurden ein handelsüblicher Bratschlauch aus Polyethylen (Toppits ${ }^{\circledR}$, Melitta Group, Minden, Deutschland), eine Drehschieberpumpe (DC 12/16 Fk, Fürgut, Tannheim, Deutschland) und ein Adsorbens (Tenax ${ }^{\circledR}$ TA 60/80 Maschenweite, TDS, Gerstel, Mühlheim, Deutschland) verwendet (Abb. 21).

Zuvor wurden die $50 \mathrm{~cm}$ langen Bratschläuche für eine Stunde bei $120{ }^{\circ} \mathrm{C}$ in einem Trockenschrank ausgeheizt, um eine mögliche Kontaminierung mit Fremd-VOC zu beseitigen. Vor jeder Messung wurden die freigelegten Feinwurzeln vor Beprobung vorsichtig vom losen Sand befreit. Der Bratschlauch wurde daraufhin um die Feinwurzeln platziert und ein Ende des Bratschlauches wurde mit einem Paketband zugeschnürt. An der Luftansaugstelle der Drehschieberpumpe wurde das Adsorbens (Tenax ${ }^{\circledR}$ TA Röhrchen) befestigt, an dem sich die von den Feinwurzeln abgegebenen VOC während des Beprobungszeitraumes anreichern konnten. Über das Adsorbens floss während der Messung ein konstanter Luftstrom von 0,2 $1 \mathrm{~min}^{-1}$. Sowohl die Luftansaugstelle mit Adsorbens als auch der Ausgang der Pumpe, welche der Luftzirkulation im Inneren des Bratschlauches dienten, wurden in 
den mit Feinwurzeln befüllten Bratschlauch gelegt. Danach wurde die noch offene Seite des Bratschlauches in ebenfalls $10 \mathrm{~cm}$ Entfernung zum Bratschlauchrand mit Paketband verschlossen, wodurch sich ein Beprobungsvolumen von 81 ergab. Die Pumpen wurden an einem $6 \mathrm{~V}$-Akku angeschlossen und für insgesamt 120 min über die Mittagszeit betrieben. Somit wurde innerhalb des Beprobungszeitraumes das Beprobungsvolumen insgesamt dreimal über das Adsorbens geleitet. Ein Datalogger (DL-120-TH,Voltcraft, Conrad Electronics SE, Hirschau, Deutschland) diente während der Messungen zur Aufzeichnung der Lufttemperatur und der Luftfeuchte. Das Messintervall betrug bei den Aufzeichnungen zehn Sekunden. Nach Versuchsende wurden die Tenax ${ }^{\circledR}$-Proben luftdicht in speziellen Glascontainern verpackt. Auf der Rückfahrt zum Institut wurden die Proben in einer Kühlbox und im Institut bis zur Analyse im GC bei $6{ }^{\circ} \mathrm{C}$ aufbewahrt. Die Analyse der Tenax ${ }^{\circledR}$-Proben wurden wie unter 3.3 .1 beschrieben mittels eines GC-MS durchgeführt.

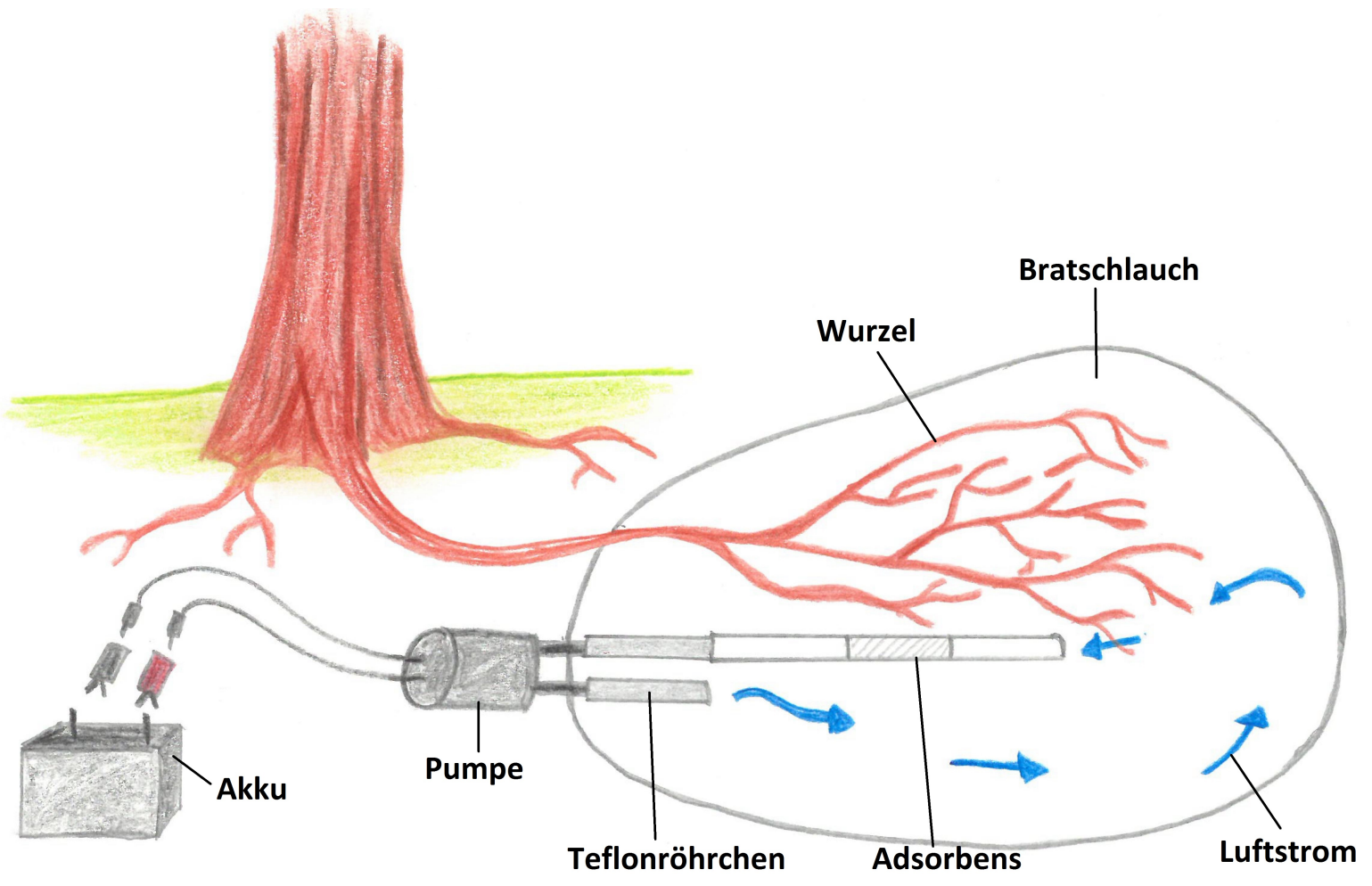

Abbildung 21: Versuchsaufbau zur Wurzel-VOC-Beprobung

\section{VOC-Auswahl}

Die VOC wurden vorläufig mit dem Programm Enhanced ChemStation Version D 02.00.275 (Agilent Technology) und der NIST Datenbank vom nationalen Institut für Standard und Technologie (NIST, Gaithersburg, USA) identifiziert. Die weitere Identifizierung wurde durch die Bestätigung des RIs mit jenen in der Literatur und durch Koelution von verdünnten Reinsubstanzen $\left(10^{-3}\right)$ durchgeführt. Diese Vorgehensweise der 
Identifizierung wurde für die VOC vorgenommen, die bei mindestens $50 \%$ aller Proben bei mindestens einem der Untersuchungsgebiete gemessen wurden.

Zusätzlich wurde das VOC-Muster der Feinwurzel mit dem VOC-Muster von Stämmen von F. sylvatica auf der Versuchsfläche Sand im Untersuchungsgebiet Unterlüß im September 2011 verglichen. Die Beprobung der Stämme erfolgte mit der StammabsaugkammerMethode nach Rachow (2012) (s. Kapitel 3.2). Bei diesen Messungen wurde als Adsorbens ebenfalls Tenax ${ }^{\circledR}$ TA Röhrchen verwendet. Die Beprobungszeit betrug zwei Stunden bei $24{ }^{\circ} \mathrm{C}$ Lufttemperatur und $82 \%$ relativer Luftfeuchtigkeit.

Um festzustellen, ob im Frühjahr die VOC ähnliche Abhängigkeiten zum Niederschlagsgradienten zeigen wie im Herbst, wurden neun VOC ausgewählt, die im Herbst deutliche Trends zeigten und die im Frühjahr in mindestens zwei Untersuchungsgebieten in mindestens $50 \%$ der Proben gefunden wurden. Für diese neun VOC (3 Aldehyde: 2-Ethylhexanal, Nonanal und Decanal; 3 Alkohole: 1-Octen-3-ol, 3-Octanol und 2-Ethylhexanol; 2 Alkane: Decan und Pentadecan; 1 Keton: 3-Octanon) wurden die Abundanzen der Hauptionen ermittelt.

\section{Statistische Auswertung}

Die statistische Auswertung wurde mit R Studio (Version 3.1.1, The R Foundation for Statistical Computing) durchgeführt. Die im Herbst erhobenen Daten wurden wegen der geringen Anzahl der Stichproben mittels des Exakten Fisher-Tests auf Unterschiede zwischen den Feinwurzel-VOC-Häufigkeiten im A- und B-Horizont und zwischen den VOC-Häufigkeiten zwischen den drei Untersuchungsgebieten getestet. Der Wilcoxon-Test wurde aufgrund nicht normal verteilter Daten zur Ermittlung von Unterschieden in den Abundanzen der Hauptionen zwischen den Untersuchungsflächen im Herbst und in den Abundanzen von neun ausgewählten VOC zwischen Herbst und Frühling verwendet. Um Unterschiede zwischen der Feinwurzel-VOC-Häufigkeit im Frühling und Herbst bei allen drei Untersuchungsgebieten zu testen, wurde der Exakte Fisher-Test verwendet. Das Signifikanzniveau lag beim Wilcoxon-Test mit Bonferroni-Korrektur $\alpha=0,017$ und beim Exakten Fisher-Test bei $\alpha=0,05$. 


\subsubsection{Ergebnisse}

\section{Auswirkung von jährlichen Niederschlagsmengen auf das Feinwurzel-VOC-Muster im Waldboden}

Die Rhizosphäre hat auf den drei Buchenstandorten insgesamt 42 VOC abgegeben, die bei mindestens einer Fläche in über $50 \%$ der Proben vorgekommen sind. Die meisten VOC gehören zu den Stoffklassen der Aldehyde (9), Alkane (9), Ketone (6), Alkohole (6) und Terpene (5) (Tab. 8). 39 der 42 VOC konnten mit authentischen Standards bestätigt werden.

Fünf VOC (Hexanal, 1-Octen-3-ol, 3-Octanon, 2-Ethylhexanol und Benzaldehyd) waren in $90 \%$ aller Wurzelproben zu finden. In allen Proben von allen drei Untersuchungsgebieten waren diese fünf VOC die dominantesten Peaks in den Chromatogrammen und jene, die zusammen mehr als 37 \% der Peakflächen im Totalionenstrom (TIC, total ion current) Chromatogramm ausmachten (Tab. 8, Abb. 22). Abb. 22 a zeigt ein Beispielchromatogramm von einer Wurzelprobe und Abb. 22 b das einer Stammprobe. Insgesamt konnten im VOC-Muster von Wurzel und Stamm 16 gemeinsame VOC identifiziert werden (Tab. 8).

Der Exakte Fisher-Test zeigte bei keinem der 42 VOC einen Unterschied in der Häufigkeit zwischen dem A- und dem B-Horizont. Dies traf in allen Untersuchungsgebieten zu. Auch das Poolen der Daten aus allen Proben vom A-Horizont und vom B-Horizont von allen drei Gebieten zusammen und der erneute Vergleich der VOC erzielte keine signifikanten Unterschiede in der Häufigkeit der einzelnen VOC zwischen den beiden Horizonten. Daher wurden die Häufigkeiten aus beiden Horizonten für das jeweilige VOC für das jeweilige Untersuchungsgebiet zusammengefasst.

Der Vergleich der Häufigkeiten der VOC zwischen den zwei Untersuchungsgebieten mit hoher jährlicher Jahresniederschlagsumme (Sellhorn) und mit geringer jährlicher Niederschlagsumme (Klötze) zeigte signifikante Unterschiede bei Undecan (Exakter FisherTest, $\mathrm{p}=0,04$ ), 2-Undecanon (Exakter Fisher-Test, $\mathrm{p}=0,01$ ) und Hexadecan (Exakter Fisher-Test, $\mathrm{p}=0,004)$. Hexadecan wurde in signifikant mehr Proben in dem Untersuchungsgebiet Klötze gefunden. Dagegen war die Häufigkeit von Undecan und 2-Undecanon signifikant höher im Untersuchungsgebiet Sellhorn.

Es konnten drei verschiedene Abhängigkeiten zum Niederschlagsgradienten für 21 VOC von 42 VOC festgestellt werden. Zwei Alkohole ((Z)-3-Hexen-1-ol und 2-Ethylhexanol), zwei Aldehyde (2-Ethylhexanal und 2-Nonenal) und ein Alkan (Decan) zeigten einen proportionalen Trend zum Niederschlagsgradienten. Die Abundanzen dieser fünf VOC waren im Untersuchungsgebiet Sellhorn mit dem meisten jährlichen Niederschlag am höchsten. Im Gegensatz dazu zeigten drei VOC (1-Octen-3-ol, 3-Octanon und 3-Octanol) einen umgekehrten proportionalen Trend zum Niederschlagsgradienten. Die Abundanzen dieser 
Tabelle 8: Häufigkeiten der identifizierten Feinwurzel-VOC

\begin{tabular}{|c|c|c|c|c|c|}
\hline & & & $\mathbf{N}=\mathbf{9}$ & $\mathbf{N}=\mathbf{9}$ & $\mathbf{N}=\mathbf{9}$ \\
\hline LRI & VOC & CAS & Sellhorn & Unterlüß & Klötze \\
\hline$<700$ & Acetylsäure & $000064-19-7$ & 6 & 8 & 5 \\
\hline 766 & 1-Pentanol \# & 000071-41-0 & 4 & 8 & 6 \\
\hline 805 & Hexanal $\# * x$ & 000066-25-1 & 9 & 9 & 7 \\
\hline 810 & 3-Cyclohepten-1-on & 001121-64-8 & 8 & 6 & 7 \\
\hline 858 & (Z)-3-Hexen-1-ol \# & 000928-96-1 & 5 & 5 & 5 \\
\hline 863 & 4-Methyloctane $\#$ & 002216-34-4 & 4 & 8 & 4 \\
\hline 864 & Ethylbenzol $^{\# x}$ & 000100-41-4 & 9 & 6 & 6 \\
\hline 888 & Heptanon \# & 000106-35-4 & 7 & 7 & 6 \\
\hline 896 & Xylen $\# x$ & $000095-47-6$ & 9 & 8 & 6 \\
\hline 901 & Nonan $\# x$ & 000111-84-2 & 7 & 4 & 5 \\
\hline 906 & Heptanal $\# x$ & $000111-71-7$ & 7 & 8 & 5 \\
\hline 920 & Anisol & 000100-66-3 & 7 & 6 & 6 \\
\hline 937 & $\alpha$-Pinen $\# x$ & 000080-56-8 & 9 & 9 & 9 \\
\hline 952 & Camphen $\# x$ & $000079-92-5$ & 8 & 8 & 6 \\
\hline 958 & 2-Ethylhexanal $\# x$ & 000123-05-7 & 9 & 8 & 6 \\
\hline 974 & Benzaldehyd $\# * x$ & $000100-52-7$ & 9 & 9 & 9 \\
\hline 981 & 1-Octen-3-ol \#* & 003391-86-4 & 9 & 8 & 8 \\
\hline 988 & $3-$ Octanon \#* & 000106-68-3 & 9 & 9 & 9 \\
\hline 997 & 3-Octanol \# & 000589-98-0 & 8 & 9 & 7 \\
\hline 1000 & Decan \# & $000124-18-5$ & 9 & 7 & 5 \\
\hline 1006 & Octanal $\# x$ & 000124-13-0 & 7 & 7 & 5 \\
\hline 1014 & 3 -Caren $\# x$ & 013466-78-9 & 9 & 8 & 7 \\
\hline 1023 & 3-Cyclohexen-1-carbonitril\# & $000100-45-8$ & 9 & 4 & 7 \\
\hline 1031 & 2-Ethylhexanol $\# * x$ & 000104-76-7 & 9 & 9 & 9 \\
\hline 1034 & Limonen $\# x$ & $000138-86-3$ & 5 & 3 & 5 \\
\hline 1096 & 1-Phenylethanon \# & 000098-86-2 & 9 & 9 & 9 \\
\hline 1094 & 2-Nonanon $\#$ & $000821-55-6$ & 2 & 7 & 6 \\
\hline 1100 & Undecan\# & $001120-21-4$ & 9 & 6 & 4 \\
\hline 1106 & Nonanal $\# x$ & 000124-19-6 & 9 & 9 & 7 \\
\hline 1154 & Campher\# & $000076-22-2$ & 5 & 8 & 6 \\
\hline 1171 & 2-Nonenal \# & $002463-53-8$ & 5 & 3 & 1 \\
\hline 1169 & Benzolcarbonsäure & 000065-85-0 & 3 & 9 & 7 \\
\hline 1197 & Naphthalen\# & $000091-20-3$ & 6 & 9 & 6 \\
\hline 1200 & Dodecan $\#$ & 000112-40-3 & 7 & 9 & 5 \\
\hline 1210 & Decanal \# & $000112-31-2$ & 9 & 8 & 6 \\
\hline 1265 & Caprolactam $\#$ & 000105-60-2 & 4 & 7 & 7 \\
\hline 1295 & 2-Undecanon\# & 000112-12-9 & 9 & 9 & 3 \\
\hline 1300 & Tridecan $\#$ & $000629-50-5$ & 6 & 9 & 9 \\
\hline 1310 & Undecanal \# & 000112-44-7 & 6 & 8 & 3 \\
\hline 1400 & Tetradecan \# & 000629-59-4 & 7 & 9 & 8 \\
\hline 1500 & Pentadecan $\# x$ & 000629-62-9 & 8 & 9 & 9 \\
\hline 1600 & Hexadecan $^{\# x}$ & $000544-76-3$ & 2 & 9 & 9 \\
\hline
\end{tabular}

$\mathrm{LRI}=$ Linearer Retentionsindex,$\overline{\mathrm{CAS}}=$ Chemical Abstracts Service Number,${ }^{*}=$ dominante VOC in den Chromatogrammen der Wurzelproben, $\#$ = durch Koelution mit authentischen Standardsubstanzen bestätigt, ${ }^{x}=$ Rot-Buchenstamm-VOC

VOC nahmen mit abnehmendem jährlichen Niederschlag in den Untersuchungsgebieten zu und hatten die höchsten Abundanzen im Untersuchungsgebiet Klötze. 13 VOC (Acetylsäure, 1-Pentanol, 4-Methyloctan, Undecan, Nonanal, Campher, Benzolcarbonsäure, Decanal, 2-Undecanon, Undecanal, Tetradecan, Pentadecan und Hexadecan) zeigten eine glockenförmige Verteilung ihrer Abundanzen mit der höchsten Abundanz in der Mitte des Niederschlagsgradienten für das Untersuchungsgebiet Unterlüß. In Abb. 23 sind für jede Abhängigkeit drei VOC dargestellt. Für den proportionalen und den glockenförmigen 

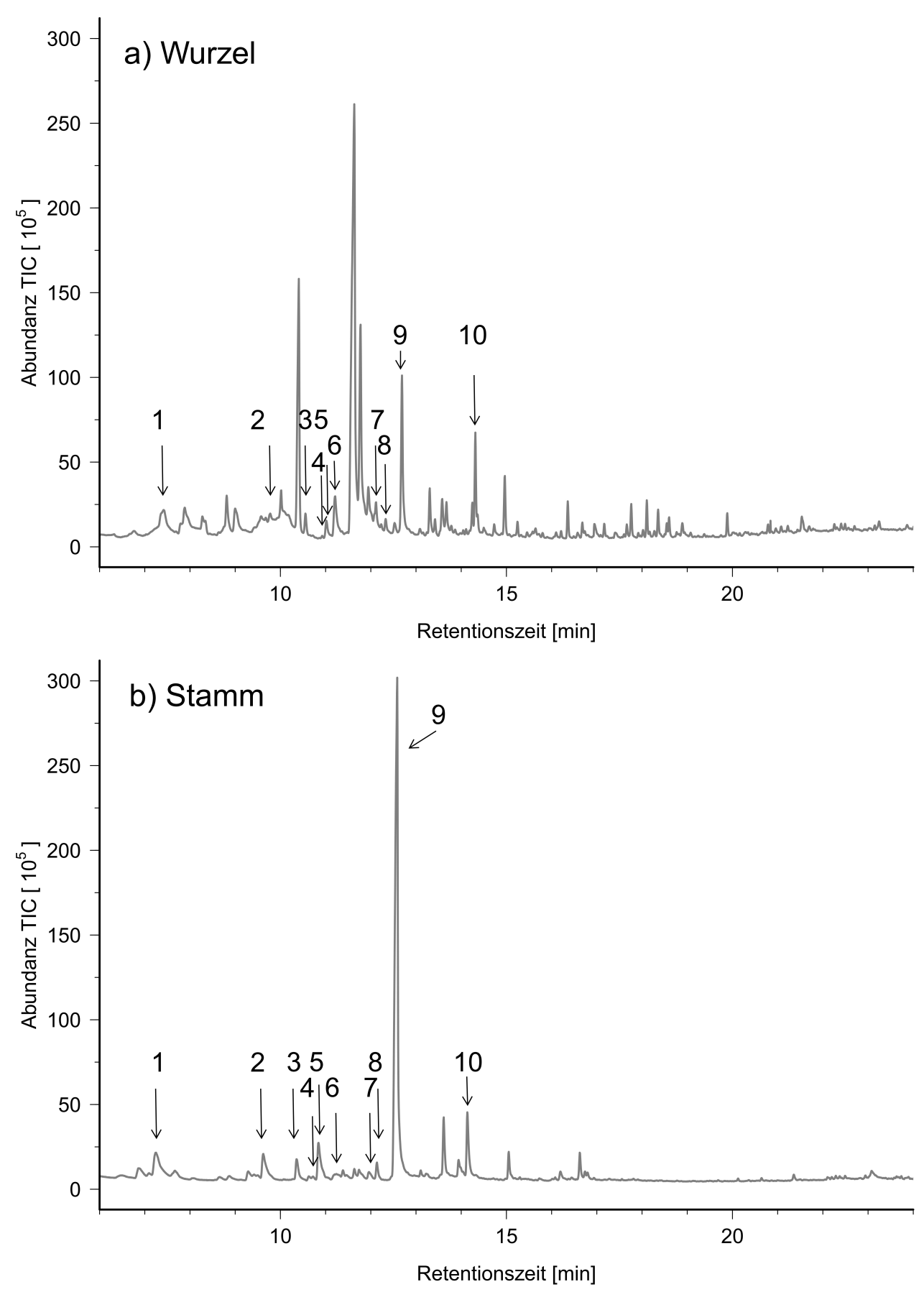

Abbildung 22: Beispielchromatogramme von einer Wurzelprobe (a) und einer Stammprobe (b) von F. sylvatica. Die Zahlen stehen für verschiedene VOC: $1=$ Hexanal, $2=$ Heptanal, $3=\alpha$-Pinen, $4=$ Camphen, $5=2$-Ethylhexanal, $6=$ Benzaldehyd, $7=$ Octanal, $8=3$-Caren, $9=2$-Ethylhexanol, $10=$ Nonanal, TIC $=$ Totalionenstrom

Verlauf sind drei VOC dargestellt, bei denen auch signifikante Unterschiede zwischen den Untersuchungsgebieten festgestellt werden konnten. 

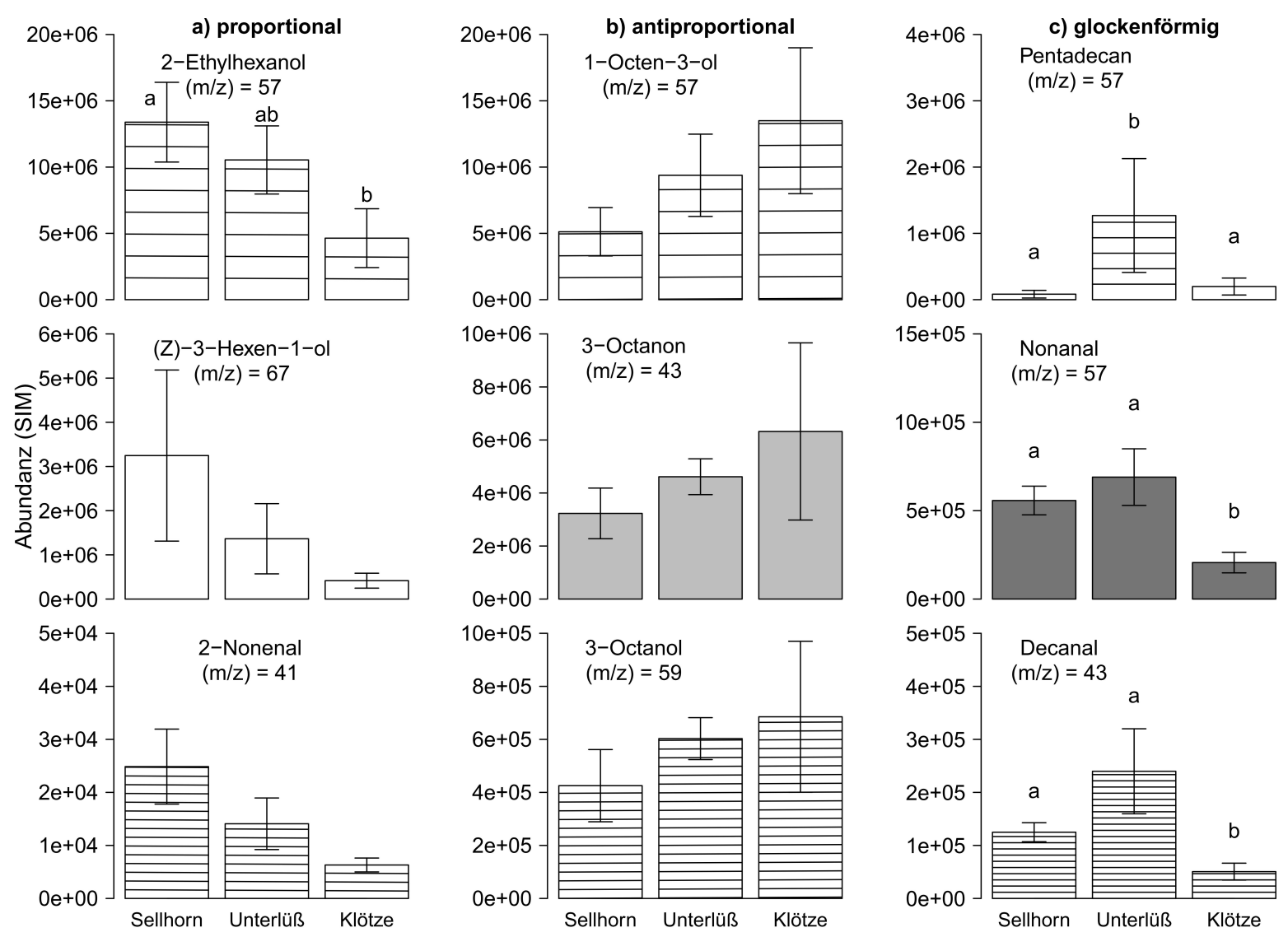

Abbildung 23: Abhängigkeiten der Feinwurzel-VOC entlang des Niederschlagsgradienten. Dargestellt sind die Abundanzen ( \pm S.e. $)$ für die Hauptionen. Gebiete mit verschiedenen Buchstaben unterscheiden sich signifikant voneinander (Wilcoxon-Test mit Bonferroni-Korrektur, $\mathrm{p} \leq 0,017$ ). SIM $=$ single ion modus

13 der 42 VOC zeigten signifikante Unterschiede in den Abundanzen des Hauptions zwischen mindestens zwei verschiedenen Untersuchungsgebieten (Wilcoxon-Test mit BonferroniKorrektur, $\mathrm{p} \leq 0,017$, Tab. 9p. 
Tabelle 9: Signifikante Unterschiede in den VOC-Abundanzen des Hauptiones zwischen den Untersuchungsgebieten (Wilcoxon-Test mit Bonferroni-Korrektur, $\mathrm{p} \leq$ $0,017)$

\begin{tabular}{|c|c|c|c|c|c|}
\hline LRI & VOC & CAS & $\mathrm{S}$ vs. $\mathrm{U}$ & $\mathbf{S}$ vs. $\mathrm{K}$ & U vs. $\mathrm{K}$ \\
\hline 906 & Heptanal $\# x$ & $000111-71-7$ & n. s. & n. s. & 0,007 \\
\hline 1000 & Decan \# & $000124-18-5$ & n. s. & 0,005 & n. s. \\
\hline 1031 & 2-Ethylhexanol $\# * x$ & 000104-76-7 & n. s. & 0,014 & n. s. \\
\hline 1106 & Nonanal $\# x$ & $000124-19-6$ & n. s. & 0,006 & 0,004 \\
\hline 1169 & Benzolcarbonsäure & 000065-85-0 & 0,001 & n. s. & 0,010 \\
\hline 1210 & Decanal\# & 000112-31-2 & n. s. & 0,010 & 0,005 \\
\hline 1265 & Caprolactam \# & $000105-60-2$ & 0,008 & n. s. & n. s. \\
\hline 1295 & 2-Undecanon $\#$ & $000112-12-9$ & 0,019 & 0,009 & 0,001 \\
\hline 1300 & Tridecan \# & 000629-50-5 & 0,001 & n. s. & n. s. \\
\hline 1310 & 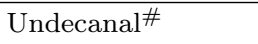 & 000112-44-7 & n. s. & n. s. & 0,014 \\
\hline 1400 & Tetradecan \# & 000629-59-4 & 0,004 & n. s. & n. s. \\
\hline 1500 & Pentadecan $\# x$ & 000629-62-9 & 0,003 & 0,019 & 0,008 \\
\hline 1600 & Hexadecan $\# x$ & $000544-76-3$ & 0,003 & 0,011 & 0,014 \\
\hline
\end{tabular}

$\mathrm{LRI}=$ Linearer Retentionsindex, CAS $=$ Chemical Abstracts Service Number,${ }^{*}=$ dominante VOC in den Chromatogrammen der Wurzelproben, $\#$ = durch Koelution mit authentischen Standardsubstanzen bestätigt, ${ }^{x}=$ Rot-Buchenstamm-VOC, n. s. = nicht signifikant, $\mathrm{S}=$ Sellhorn, $\mathrm{U}=$ Unterlüß, $\mathrm{K}=\mathrm{Klötze}$ 


\section{Saisonale Unterschiede des Feinwurzel-VOC-Musters}

Es wurden im Herbst und im Frühjahr jeweils neun VOC-Proben von Feinwurzeln auf den Sandflächen der Untersuchungsgebieten Sellhorn, Unterlüß und Klötze analysiert.

Im Frühjahr konnten bis auf (Z)-3-Hexen-1-ol und 4-Methyloctan alle im Herbst identifizierten 42 VOC in mindestens einem Untersuchungsgebiet in der Hälfte aller Proben gefunden werden (Tab. 10). Es konnten im Frühjahr darüber hinaus keine zusätzlichen VOC gefunden werden.

Die fünf VOC Hexanal, 1-Octen-3-ol, 3-Octanon, 2-Ethylhexanol und Benzaldehyd, welche in den Herbstmessungen in $90 \%$ der Wurzelproben vorhanden waren, konnten in $80 \%$ der Proben im Frühjahr gefunden werden (Tab. 10).

Elf VOC unterscheiden sich in ihrem Vorkommen in mindestens einem Untersuchungsgebiet zwischen Herbst und Frühling signifikant (Tab. 11). Neun der elf VOC wurden in signifikant mehr Proben im Herbst als im Frühling gefunden. Sechs dieser neun VOC wurden nur im Herbst in mindestens einem der Untersuchungsgebiet gemessen. 2-Nonenal wurde im Herbst nur in Sellhorn und Unterlüß, 4-Methyloctan und 3-Octanol nur in Unterlüß, Decan und Limonen nur in Klötze und (Z)-3-Hexen-1-ol nur in Sellhorn und Unterlüß gemessen. Zwei VOC (2-Nonanon und Benzolcarbonsäure) wurden dagegen im Frühling im Untersuchungsgebiet Sellhorn in signifikant mehr Proben gefunden (Tab. 11).

Nicht nur für die Häufigkeiten, sondern auch in den Abundanzen entlang des Niederschlagsgradienten konnten Unterschiede zwischen Herbst und Frühling für einzelne VOC festgestellt werden. 2-Ethylhexanol, 2-Ethylhexanal und Decan hatten im Herbst einen proportionalen Trend zum Niederschlagsgradienten (Abb. 24). Im Frühjahr zeigte das Alkan Decan den gleichen Trend. Dagegen wies 2-Ethylhexanol im Frühjahr einen umgekehrt proportionalen Trend auf. 2-Ethylhexanal zeigte auf den Flächen Sellhorn und Unterlüß ebenfalls einen umgekehrt proportionalen Trend, welcher auf der Fläche Klötze nicht fortgesetzt wurde.

Die VOC 1-Octen-3-ol, 3-Octanon und 3-Octanol zeigten im Herbst einen umgekehrt proportionalen Trend zum Niederschlagsgradienten. Im Frühling war dieser Trend nicht erkennbar. Alle drei VOC hatten ihre geringste mittlere Abundanz im Untersuchungsgebiet Unterlüß. Auffallend sind hier die signifikanten Unterschiede von 3-Octanon und 3-Octanol im Untersuchungsgebiet Sellhorn. Hier wurden im Frühjahr beide VOC signifikant mehr abgegeben (Abb. 24). Dagegen wurde 1-Octen-3-ol im Frühjahr signifikant mehr als am Standort Klötze freigesetzt.

Pentadecan, Nonanal und Decanal hatten im Herbst ihre höchste mittlere Abundanz im Untersuchungsgebiet Unterlüß. Auch das Alkan Pentadecan erreichte im Frühjahr die höchste Abundanz in Unterlüß. Zwar konnte im Frühjahr für Nonanal und Decanal kein eindeutiger Trend festgestellt werden, dafür konnten signifikant höhere Abundanzen für 
das Untersuchungsgebiet Klötze im Frühjahr gemessen werden (Abb. 24).

Tabelle 10: Häufigkeiten der identifizierten Feinwurzel-VOC für Herbst und Frühling

\begin{tabular}{|c|c|c|c|c|c|c|c|c|}
\hline \multirow[b]{2}{*}{ LRI } & \multirow[b]{2}{*}{ VOC } & \multirow[b]{2}{*}{ CAS } & \multicolumn{2}{|c|}{$\begin{array}{c}\mathbf{N}=\mathbf{9} \\
\text { Sellhorn }\end{array}$} & \multicolumn{2}{|c|}{$\begin{array}{c}\mathbf{N}=\mathbf{9} \\
\text { Unterlüß }\end{array}$} & \multicolumn{2}{|c|}{$\begin{array}{l}\mathbf{N}=9 \\
\text { Klötze }\end{array}$} \\
\hline & & & $\mathrm{H}$ & $\mathrm{F}$ & $\mathrm{H}$ & $\mathrm{F}$ & $\mathrm{H}$ & $\mathrm{F}$ \\
\hline$<700$ & Acetylsäure\# & 000064-19-7 & 6 & 5 & 8 & 8 & 5 & 4 \\
\hline 766 & 1-Pentanol $\#$ & 000071-41-0 & 4 & 7 & 8 & 1 & 6 & 4 \\
\hline 805 & Hexanal $^{\# * x}$ & 000066-25-1 & 9 & 9 & 9 & 7 & 7 & 7 \\
\hline 810 & 3-Cyclohepten-1-on & 001121-64-8 & 8 & 5 & 6 & 4 & 7 & 8 \\
\hline 858 & (Z)-3-Hexen-1-ol\# & 000928-96-1 & 5 & 0 & 5 & 0 & 5 & 1 \\
\hline 863 & 4-Methyloctan\# & 002216-34-4 & 4 & 4 & 8 & 0 & 4 & 0 \\
\hline 864 & Ethylbenzol $\# x$ & 000100-41-4 & 9 & 9 & 6 & 9 & 6 & 9 \\
\hline 888 & Heptanon $\#$ & 000106-35-4 & 7 & 9 & 7 & 3 & 6 & 8 \\
\hline 896 & Xylen $\# x$ & $000095-47-6$ & 9 & 9 & 8 & 9 & 6 & 9 \\
\hline 901 & Nonan\# $\# x$ & 000111-84-2 & 7 & 2 & 4 & 5 & 5 & 3 \\
\hline 906 & Heptanal $\# x$ & 000111-71-7 & 7 & 9 & 8 & 5 & 5 & 8 \\
\hline 920 & Anisol & 000100-66-3 & 7 & 5 & 6 & 8 & 6 & 4 \\
\hline 937 & $\alpha$-Pinen $\# x$ & 000080-56-8 & 9 & 7 & 9 & 8 & 9 & 5 \\
\hline 952 & Camphen $\# x$ & 000079-92-5 & 8 & 4 & 8 & 5 & 6 & 2 \\
\hline 958 & 2-Ethylhexanal $\# x$ & 000123-05-7 & 9 & 9 & 8 & 8 & 6 & 6 \\
\hline 974 & Benzaldehyd $\# * x$ & $000100-52-7$ & 9 & 9 & 9 & 9 & 9 & 8 \\
\hline 981 & 1-Octen-3-ol \#* & 003391-86-4 & 9 & 6 & 8 & 8 & 8 & 8 \\
\hline 988 & 3-Octanon $\# *$ & 000106-68-3 & 9 & 9 & 9 & 9 & 9 & 8 \\
\hline 997 & 3-Octanol \# & 000589-98-0 & 8 & 7 & 9 & 0 & 7 & 7 \\
\hline 1000 & Decan\# & 000124-18-5 & 9 & 5 & 7 & 7 & 5 & 0 \\
\hline 1006 & Octanal $^{\# x}$ & 000124-13-0 & 7 & 9 & 7 & 9 & 5 & 8 \\
\hline 1014 & 3 -Caren $\# x$ & 013466-78-9 & 9 & 8 & 8 & 9 & 7 & 6 \\
\hline 1023 & 3-Cyclohexen-1-carbonitril \# & $000100-45-8$ & 9 & 2 & 4 & 3 & 7 & 4 \\
\hline 1031 & 2-Ethylhexanol \#*x & 000104-76-7 & 9 & 9 & 9 & 9 & 9 & 8 \\
\hline 1034 & Limonen $\# x$ & 000138-86-3 & 5 & 5 & 3 & 2 & 5 & 0 \\
\hline 1096 & 1-Phenylethanon \# & 000098-86-2 & 9 & 9 & 9 & 8 & 9 & 9 \\
\hline 1094 & 2-Nonanon $\#$ & 0000821-55-6 & 2 & 9 & 7 & 6 & 6 & 7 \\
\hline 1100 & Undecan $\#$ & 001120-21-4 & 9 & 7 & 6 & 3 & 4 & 5 \\
\hline 1106 & Nonanal $^{\# x}$ & 000124-19-6 & 9 & 9 & 9 & 8 & 7 & 8 \\
\hline 1154 & Campher\# & $000076-22-2$ & 5 & 9 & 8 & 8 & 6 & 1 \\
\hline 1171 & 2-Nonenal \# & 002463-53-8 & 5 & 0 & 3 & 0 & 1 & 6 \\
\hline 1169 & Benzolcarbonsäure & $000065-85-0$ & 3 & 9 & 9 & 9 & 7 & 7 \\
\hline 1194 & 2-Decanon & 000693-54-9 & 6 & 5 & 9 & 7 & 6 & 6 \\
\hline 1200 & Dodecan $\#$ & 000112-40-3 & 7 & 7 & 9 & 8 & 5 & 9 \\
\hline 1210 & Decanal \# & 000112-31-2 & 9 & 9 & 8 & 9 & 6 & 8 \\
\hline 1265 & Caprolactam\# & 000105-60-2 & 4 & 0 & 7 & 5 & 7 & 1 \\
\hline 1295 & 2-Undecanon\# & 000112-12-9 & 9 & 8 & 9 & 7 & 3 & 7 \\
\hline 1300 & Tridecan $\#$ & 000629-50-5 & 6 & 9 & 9 & 9 & 9 & 8 \\
\hline 1310 & Undecanal \# & 000112-44-7 & 6 & 8 & 8 & 5 & 3 & 8 \\
\hline 1400 & Tetradecan \# & 000629-59-4 & 7 & 9 & 9 & 9 & 8 & 8 \\
\hline 1500 & Pentadecan $\# x$ & 000629-62-9 & 8 & 9 & 9 & 9 & 9 & 8 \\
\hline 1600 & Hexadecan $\# x$ & 000544-76-3 & 2 & 9 & 9 & 8 & 9 & 7 \\
\hline
\end{tabular}

$\mathrm{H}=$ Herbst, $\mathrm{F}=$ Frühling, LRI $=$ Linearer Retentionsindex, CAS $=$ Chemical Abstracts Service Number, ${ }^{*}=$ dominante VOC in den Chromatogrammen der Wurzelproben, $\#=$ durch Koelution mit authentischen Standardsubstanzen bestätigt, ${ }^{x}=$ Rot-Buchenstamm-VOC 
Tabelle 11: Signifikante saisonale Unterschiede in den VOC-Häufigkeiten der Untersuchungsgebiete (Exakter Fisher-Test, $\mathrm{p} \leq 0,05$ )

\begin{tabular}{|c|c|c|c|c|c|}
\hline LRI & VOC & CAS & $\begin{array}{r}\mathrm{N}=9 \\
\text { SH vs. SF }\end{array}$ & $\begin{array}{r}\mathrm{N}=9 \\
\text { UH vs. UF }\end{array}$ & $\begin{array}{r}N=9 \\
\text { KH vs. KF }\end{array}$ \\
\hline 766 & 1-Pentanol & 000071-41-0 & n. s. & 0,005 & n. s. \\
\hline 858 & (Z)-3-Hexen-1-ol & 000928-96-1 & 0,035 & 0,035 & n. s. \\
\hline 863 & 4-Methyloctan & 002216-34-4 & n. s. & 0,001 & n. s. \\
\hline 997 & 3-Octanol & 000589-98-0 & n. s. & $<0,001$ & n. s. \\
\hline 1000 & Decan & 000124-18-5 & n. s. & n. s. & 0,035 \\
\hline 1023 & 3-Cyclohexen-1-carbonitril & $000100-45-8$ & 0,004 & n. s. & n. s. \\
\hline 1034 & Limonen & 000138-86-3 & n. s. & n.s. & 0,035 \\
\hline 1094 & 2-Nonanon & $000821-55-6$ & 0,004 & n. s. & n. s. \\
\hline 1171 & 2-Nonenal & 002463-53-8 & 0,035 & n. s. & n. s. \\
\hline 1169 & Benzolcarbonsäure & 000065-85-0 & 0,012 & n. s. & n. s. \\
\hline 1265 & Caprolactam & 000105-60-2 & n. s. & n. s. & 0,018 \\
\hline
\end{tabular}

$\mathrm{S}=$ Sellhorn, $\mathrm{U}=$ Unterlüß, $\mathrm{K}=$ Klötze, $\mathrm{H}=$ Herbst, $\mathrm{F}=$ Frühling, LRI = Linearer Retentionsindex, CAS $=$ Chemical Abstracts Service Number, n. s. = nicht signifikant
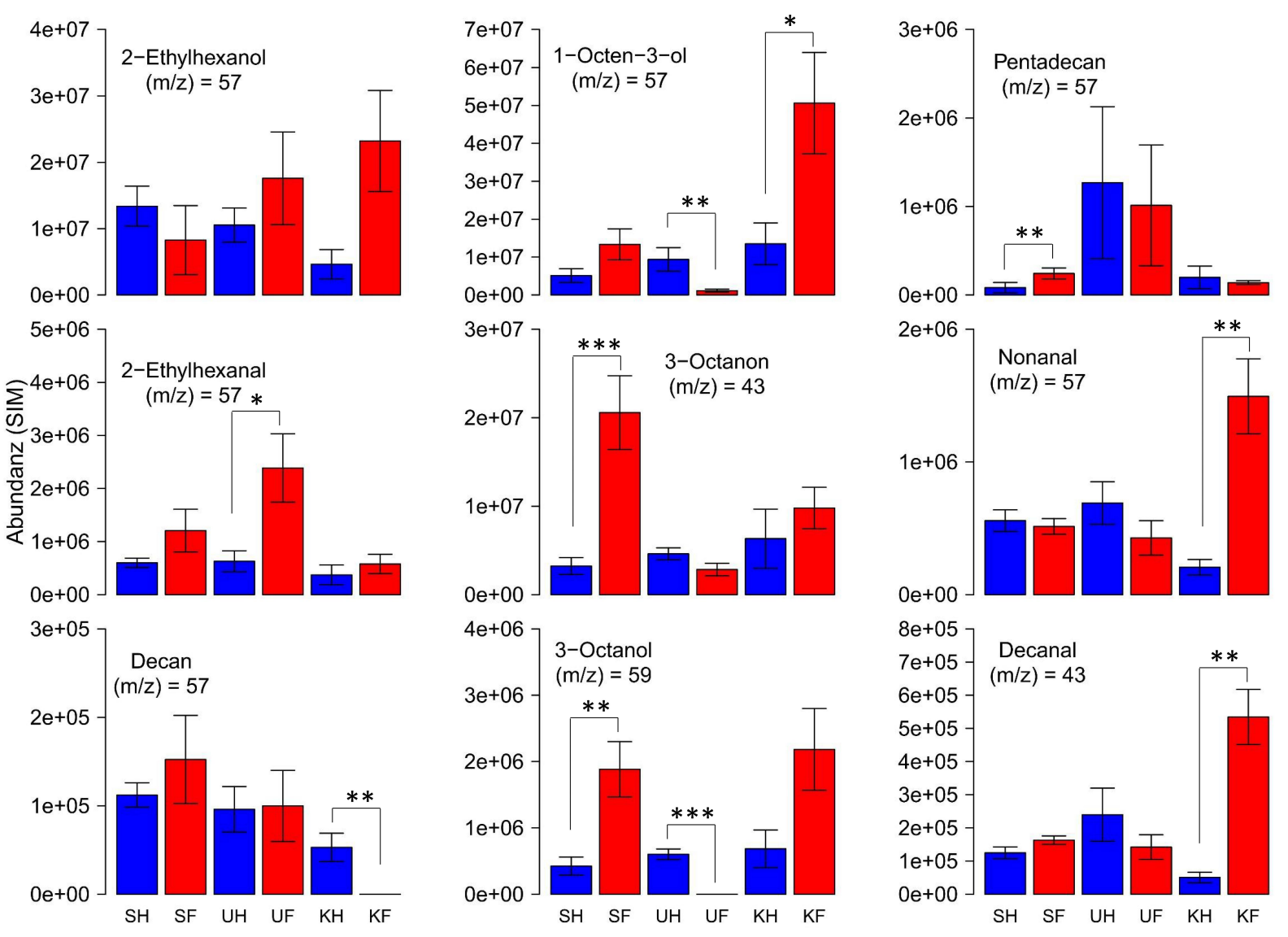

Abbildung 24: Signifikante saisonale Unterschiede in den Abundanzen ( \pm s. e.) der Hauptionen von ausgewählten VOC für Herbst $(\mathrm{H}$, blau) und Frühling (F, rot). Wilcoxon-Test: ${ }^{*}=\mathrm{p} \leq 0,05,{ }^{* *}=\mathrm{p} \leq 0,01,{ }^{* * *}=\mathrm{p} \leq 0,001$. $\mathrm{S}=$ Sellhorn, $\mathrm{U}=$ Unterlüß, $\mathrm{K}=$ Klötze 


\subsubsection{Diskussion}

\section{Auswirkung von jährlichen Niederschlagsmengen auf das Feinwurzel-VOC-Muster im Waldboden}

Es ist bekannt, dass Wurzeln ein breites Spektrum an organischen Substanzen abgeben [241]. Diese Studie hat erstmalig das VOC-Muster von Feinwurzeln in Buchenbeständen im Freiland untersucht und konnte erstmalig 42 VOC identifizieren, die von den Feinwurzeln und daran haftenden Mikroorganismen freigesetzt wurden. Aufgrund der Komplexität der Interaktionen im Waldboden und in der Rhizosphäre (s. Kapitel 4.1.2) ist es schwierig, einem VOC eine eindeutige Quelle zuzuordnen, da viele Organismen im Waldboden ähnliche VOC emittieren können. Die Zuordnung der identifizierten VOC zu den verschiedenen möglichen Emittern (Feinwurzeln, Pilzen und Bakterien) erfolgte daher durch den Vergleich von Literatur (Anhang A.4) und eigenen Stamm-VOC-Messungen von F. sylvatica. Für viele der gemessenen VOC kamen dabei mehr als ein möglicher Akteur in Frage.

Ein Vergleich der identifizierten Feinwurzel-VOC mit im Herbst gemessenen StammVOC von F. sylvatica ergab 16 gemeinsame VOC. Diese 16 VOC können insofern mögliche von F. sylvatica produzierte Feinwurzel-VOC sein (Tab. 8, Abb. 25 a). Unter diesen 16 VOC gehören die Aldehyde Hexanal und Nonanal sowie die Terpene $\alpha$-Pinen, 3-Caren und Limonen, welche bereits zuvor schon von anderen Studien als VOC von F. sylvatica beschrieben wurden, die von den oberirdischen Organen von $F$. sylvatica freigesetzt werden [39, 40, 115, 126 128, 142]. Studien belegen, dass zehn von den 16 VOC bereits als Wurzel-VOC bekannt sind (Abb. 25 a). Zu diesen zehn VOC gehören die drei Terpene $\alpha$-Pinen, Limonen und Camphen, Hauptkomponenten des Wurzel-VOC-Musters von Pinus spp. 211], und sieben weitere VOC (Hexanal, Heptanal, Octanal, Nonanal, 3-Caren, 2-Ethylhexanol und Pentadecan), die bekannt dafür sind von Pflanzenwurzeln emittiert zu werden 224, 234,242. Diese zehn VOC deuten darauf hin, dass die Feinwurzeln von F. sylvatica als VOC-Quelle in Frage kommen könnten.

34 der 42 identifizierten VOC konnten durch den Literaturabgleich den mikrobiellen VOC (mVOC) zugeordnet werden (Abb. 25 b). 13 dieser VOC konnten sowohl mit der Gruppe der Bodenbakterien als auch der Gruppe der Bodenpilze in Verbindung gebracht werden (Abb. 25 b). Sechs VOC (Benzaldehyd, 2-Ethylhexanol, Nonanal, Decanal, Tetradecan und Pentadecan), welche laut Literatur mit beiden Gruppen der Mikroorganismen zusammenhängen, konnten konstant in allen Proben in allen Untersuchungsgebieten (in mindestens $2 / 3$ der Proben bei jedem Untersuchungsgebiet) gefunden werden (Tab. 8). Dies deutet darauf hin, dass Mikroorganismen an Feinwurzeln von F. sylvatica einen Beitrag zu dem VOC-Muster in der Rhizosphäre leisten können.

Zusätzlich zum Literaturvergleich können auch die unterschiedlichen Abhängigkeiten 


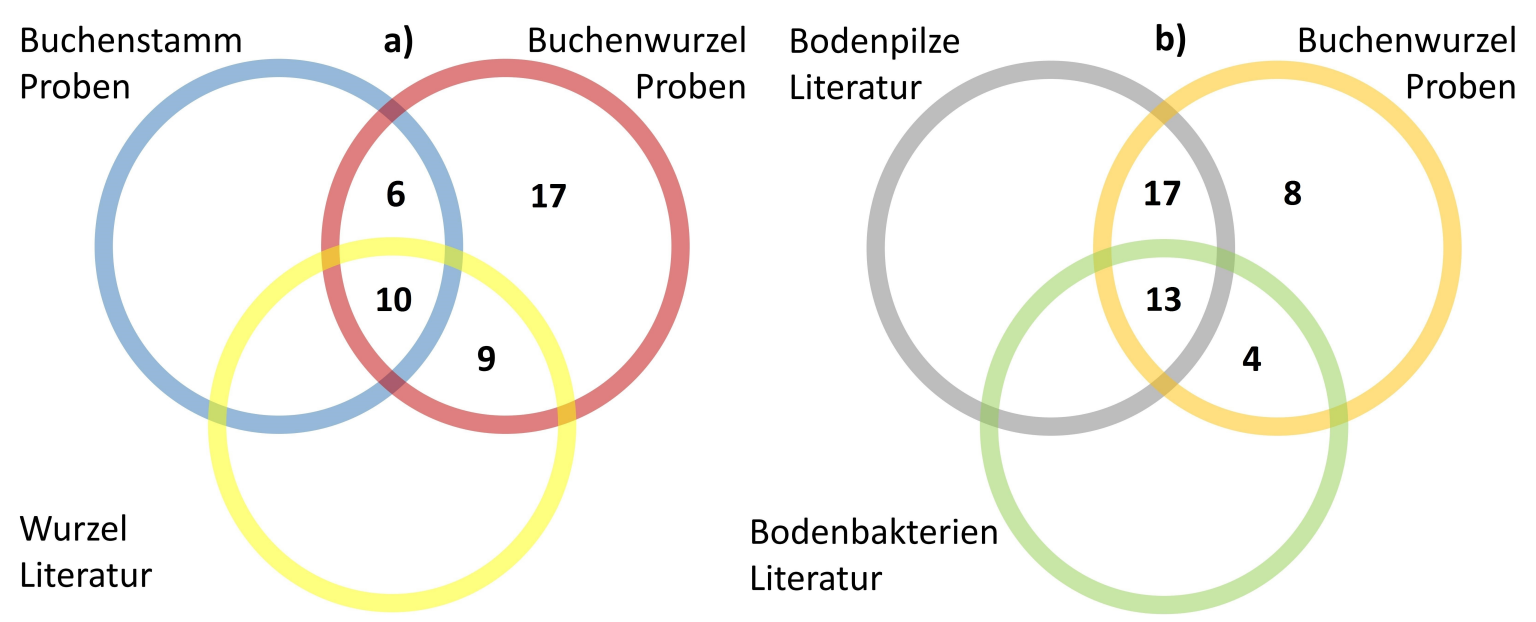

Abbildung 25: Zuordnung der gemessenen VOC zu (a) buchenbürtigen VOC und (b) mikrobiellen VOC (mVOC).

zum Niederschlagsgradienten auf mögliche Organismengruppen, die diese VOC während der Messungen abgegeben haben, und auf mögliche Interaktionen im Waldboden hindeuten.

Die Abundanzen der VOC 1-Octen-3-ol und 3-Octanon, welche für den typischen Pilzduft verantwortlich sind 243, 244, und 3-Octanol, ebenfalls als pilzbürtiges VOC bekannt [243], zeigten einen umgekehrt proportionalen Trend zum Niederschlagsgradienten (Abb. 23 b). Die Abundanzen dieser drei VOC nahmen mit abnehmender jährlicher Niederschlagsmenge zu und könnten auf eine Pilzaktivität in der Rhizosphäre in Gebieten mit geringem jährlichem Niederschlag hindeuten. Dies könnte eine höhere Wachstumsaktivität von Mykorrhizapilzen vermuten lassen, welche Bäume auf trockeneren Standorten mit Wasser versorgen 245. Die Untersuchung der Arbeitsgruppe Pflanzenökologie und Ökosytemforschung bestätigt diese Vermutung, wonach im Untersuchungsgebiet Klötze mit geringerem Jahresniederschlag (>700 mm) mehr Wurzelspitzen $(96,7 \%)$ von F. sylvatica im Herbst mykorriziert waren 105 .

Einen proportionalen Trend zur jährlichen Niederschlagsmenge zeigten die VOC (Z)3-Hexen-1-ol, 2-Ethylhexanal, 2-Ethylhexanol, 2-Nonenal und Decan (Abb. 23 a). (Z)-3Hexen-1-ol und (E)-2-Nonenal sind Abbauprodukte der Linolensäure, welche als Markersubstanz für den Feinwurzelgehalt in der Nahrung der Mesofauna im Boden verwendet wird 246]. 2-Ethylhexanol stammt möglicherweise von im Boden befindlichen Weichmachern wie z. B. Diphenyl-2-Ethylhexylphosphat und Di(2-Ethylhexyl)-Phthalat, welche sich allgegenwärtig im Boden befinden. Durch die Hydrolase-Aktivität der Pflanzenwurzeln könnte 2-Ethylhexanol von Weichmachern freigesetzt worden sein 247. Diese Hydrolasen könnten von den Pflanzenwurzeln durch Verteidigung ausgelöst worden sein, was auf eine zunehmende Verteidigungsaktivität der Pflanzenwurzeln in Gebieten mit hohen 
jährlichen Niederschlagsmengen hindeutet.

Vier VOC, die einen glockenförmigen Trend zur Niederschlagsmenge zeigen, stehen in Verbindung mit Bakterien (Undecan, Undecanal, 2-Undecanon) und mit Pilzen (1-Phenylethanon) (Anhang A.4). Dies könnte ein Hinweis auf eine zunehmende Interaktion zwischen Pflanzenwurzeln und Mikroorganismen wie Bakterien und Pilzen im Untersuchungsgebiet Unterlüßs sein. Unterlüß wies in der Untersuchung von Meier et al. (2018) die höchste Feinwurzelbiomasse von allen drei Untersuchungsgebieten auf (Unterlüß $1046 \mathrm{~g} \mathrm{~m}^{-1}$; Klötze $714 \mathrm{~g} \mathrm{~m}^{-1}$; Sellhorn $541 \mathrm{~g} \mathrm{~m}^{-1}$ ) [105]. Es kann sein, dass eine höhere Feinwurzelbiomasse im Waldboden auch eine erhöhte Interaktion über VOC notwendig macht. Es ist bekannt, dass durch die Freisetzung der Aldehyde Nonanal und Decanal Mykorrhizahelferbakterien das Wachstum pathogener Pilze hemmen können und das Wachstum von Mykorrhizapilzen unterstützen [229]. Dies würde ebenfalls dafür sprechen, dass im Untersuchungsgebiet Unterlüß eine verstärkte Interaktion zwischen Wurzeln und Mikroorganismen stattfindet.

Die Aldehyde Nonanal und Decanal sind des Weiteren als Trockenstress-VOC bei Pflanzen bekannt 161. Dies kann auf einen periodischen Trockenstress im Sommer hindeuten, der in den drei Untersuchungsgebieten unterschiedlich stark ausgeprägt war und die Gesamtfitness der Bäume auf den Flächen beeinflusst hat. Dies deckt sich mit den Ergebnissen der Arbeitsgruppe Pflanzenökologie und Ökosystemforschung, die für Unterlüß die längste Trockenperiode im Sommer 2013 von 110 Tagen gemessen hat, an denen der Bodenwassergehalt im Bodenprofil auf $40 \%$ seines eigentlichen maximalen Gehalt gesunken war (Sellhorn 84 Tage, Klötze 90 Tage) 105. Dies unterstützt die Vermutung, dass Stressmarker in Form von VOC auf die Gesamtfitness von F. sylvatica hinweisen können.

Ebenfalls als Infochemikalie könnte (Z)-3-Hexen-1-ol fungieren. (Z)-3-Hexen-1-ol, bekannt als Markersubstanz für Pflanzenvitalität [248, zeigte den Trend, mit zunehmenden Gradienten auch vermehrt von den Feinwurzeln emittiert zu werden. Somit könnte (Z)-3Hexen-1-ol als Anzeiger für Vitalität von Feinwurzeln im Boden dienen.

\section{Saisonale Unterschiede des Feinwurzel-VOC-Musters}

Die Messungen der Feinwurzlen wurden sowohl im Herbst als auch im Frühjahr zur selben Tageszeit in der Mittagszeit durchgeführt. Während der Messungen im Frühjahr war der Boden feucht, da es teilweise vor oder während der Beprobung (in Sellhorn und Unterlüß) geregnet hatte.

Beim Vergleich der Messungen von Herbst und Frühahr sind jahreszeitliche Schwankungen in der Häufigkeit der VOC und in den Abundanzen zu erkennen (Tab. 11 und 24). Da die Umweltbedingungen im Hinblick auf Temperatur und Luftfeuchte zu beiden Messkampagnen ähnlich waren, ist anzunehmen, dass die Änderungen der VOC-Muster und -Emissionen mit den jahreszeitlichen Schwankungen im Biorhythmus von F. sylva- 
tica zusammenhängen könnten. F. sylvatica besitzt im Mai größtenteils voll entwickelte und assimilierende Blätter, während im Oktober das Laub anfängt sich zu verfärben oder schon abgeworfen ist und F. sylvatica auf ihre Reserven zurückgreifen muss.

Es wurden im Vergleich zum Herbst im Frühjahr keine neuen VOC in den Bodenproben gefunden. Dies lässt vermuten, dass sich die Zusammensetzung des VOC-Musters der Feinwurzeln im Gegensatz zum VOC-Muster der Stämme kaum im Jahresverlauf verändert (s. Kapitel 3.4). Eine mögliche Ursache könnten die Umweltbedingungen sein. Oberirdisch reagieren die Pflanzenorgane unmittelbar auf kurzfristige Veränderungen der Umgebungsbedingungen wie Lufttemperatur, Luftfeuchte und Strahlung 249, 250]. Besonders die Temperatur ist ein wesentlicher Faktor, der die Abgabe von VOC beeinflussen kann 251. Der Waldboden reagiert gegenüber kurzfristigen Veränderungen der Lufttemperatur gedämpft. Die Bodentemperatur passt sich einer schnellen Veränderung der Lufttemperatur nur langsam an 252]. Dadurch ist der Waldboden nur geringeren Temperaturschwankungen ausgesetzt, was zur Folge haben könnte, dass das VOC-Muster der Feinwurzeln saisonal ähnlich ist.

Auch wenn sich in der Zusammensetzung des Wurzel-VOC-Musters von F. sylvatica keine Unterschiede zeigten, so konnten signifikante Unterschiede in den Häufigkeiten von elf VOC zwischen Herbst und Frühling und zwischen den verschiedenen Untersuchungsgebieten festgestellt werden. Im Herbst konnten vorwiegend höhere Häufigkeiten der VOC identifiziert werden als im Frühjahr (Tab. 11). Interessant ist dabei das VOC (Z)-3-Hexen1-ol. Dieses wurde im Herbst in allen drei Untersuchungsgebieten in jeweils fünf der neun Proben gefunden. Im Frühjahr konnte es nur einmal in einer Probe aus Klötze nachgewiesen werden (Tab. 10). (Z)-3-Hexen-1-ol zeigte zudem im Herbst eine proportionale Abhängigkeit zum Niederschlagsgradienten (Abb. 23 b) und wurde zuvor als Markersubstanz für den Feinwurzelgehalt in der Nahrung der Mesofauna im Boden diskutiert. Dies könnte daran liegen, dass im Frühjahr die Aktivität der Mesofauna gerade erst begonnen hat. Des Weiteren ist bekannt, dass der Insektenfraß die Abgabe von (Z)-3-Hexen-1-ol bei Pflanzen, besonders der Blattorgane, stimuliert [253]. Was Pflanzen über ihre Blätter abgeben können, können sie möglicherweise auch über die Wurzeln als Abwehrreaktion emittieren. Dies würde ebenfalls darauf hindeuten, dass die Population der Mesofauna im Herbst größer gewesen sein könnte als im Frühjahr.

Vier VOC (Anisol, 1-Octen-3-ol, 3-Octanon und 2-Ethylhexanol) sind dafür bekannt von verletzten Wurzeln und drei VOC (Nonanal, Campher und Decanal) sind dafür bekannt sowohl von unverletzten als auch von verletzten Wurzeln von Eichen (Quercus petraea x Quercus robur) emittiert und vom Engerling von M. hippocastani wahrgenommen zu werden [42. In den Verhaltensversuchen von Weissteiner et al. (2012) konnte für Anisol eine direkte Bewegung der Engerlinge im Erdboden zur Duftquelle nachgewiesen 
werden. Anisol wird in der Untersuchung von Weissteiner et al. (2012) als mögliches VOC diskutiert, welches dem bodenlebendem Engerling bei der Orientierung dienen kann [42]. Die in den Proben identifizierten VOC könnten somit möglicherweise nicht nur bei den Interaktionen zwischen Feinwurzeln und der Mikrofauna dienen, sondern auch bei den Interaktionen mit der Mesofauna.

Die Abundanzen von 2-Ethylhexanol zeigten im Frühjahr einen gegenläufigen Trend zum Herbst mit der niedrigsten mittleren Abundanz in Sellhorn und der höchsten mittleren Abundanz in Klötze. 2-Ethylhexanol konnte durch Literaturabgleich den Bodenpilzen ebenso wie den Bodenbakterien und den Wurzeln als Emitter zugeordnet werden (Anhang A.4. Dieses VOC könnte das Ergebnis einer zunehmenden Interaktion zwischen allen drei Gruppen (Wurzel, Bakterien und Pilzen) sein, was auf eine erhöhte Konkurrenz- und Symbiose-Aktivität im Gebiet mit niedrigem jährlichen Niederschlag zurückzuführen sein könnte. 


\subsection{Entwicklung einer Apparatur für die Untersuchung der Bodenbeweglichkeit von Wurzel-VOC 3}

Es ist nicht nur wichtig, welche VOC über Feinwurzeln emittiert werden, sondern auch, ob diese von Bodenorganismen über eine gewisse Entfernung wahrgenommen werden können. Bodenorganismen müssen sich im Boden und in der Rhizosphäre verschiedenen Herausforderungen stellen. Da die visuelle Orientierung im Boden stark begrenzt ist, helfen VOC den Bodenorganismen beim Auffinden ihrer Wirtspflanzen. Wurzel-VOC können nicht nur bei der Orientierung eine wichtige Rolle spielen, sondern dienen vermutlich auch als Kommunikationsmittel zwischen den Bodenorganismen [254].

Die Diversität der chemischen Substanzen der Wurzel-Exudate ist groß und variiert zwischen geringem und hohem Molekulargewicht [255]. Wurzel-Exsudate mit großem Molekulargewicht sind vorwiegend wasserlöslich und können von den Bodenorganismen durch den Geschmackssinn wahrgenommen werden. Wurzel-Exsudate mit geringem Molekulargewicht sind dagegen schlecht wasserlöslich und können aufgrund ihres hohen Dampfdruckes schnell die Gasphase des Bodens durchqueren.

Die Aufenthaltsdauer und Beweglichkeit von VOC im Boden ist abhängig von ihrer chemischen Struktur und von den Aggregatszuständen im Boden. Aufgrund des hohen Dampfdruckes bewegen sich VOC vorwiegend durch Diffusion durch den Boden [256], diese ist in der gasförmigen Phase abhängig vom Porenvolumen.

Im Gegensatz zum atmosphärischen Transport von VOC ist nicht nur wenig über das VOC-Muster im Boden bekannt, sondern auch über deren Mobilität im Boden. Bisherige Untersuchungen haben sich bisher damit beschäftigt, wie sich die Adsorption verschiedener VOC an Mineralboden abhängig vom Wassergehalt, dem Bodentyp, der Stoffklasse und der Huminsäure verändert 257,258.

In dieser Studie wird die Entwicklung einer Apparatur zur Messung der Bodenbeweglichkeit von Wurzel-VOC beschrieben, in dessen Rahmen eine Masterarbeit „Entwicklung einer Apparatur für die Untersuchung der Bodenbeweglichkeit von Wurzelvolatilen“ von Alisa Klamm verfasst wurde.

${ }^{3}$ Die Entwicklung der Apparatur wurde modifiziert unter dem Titel „New experimental setup to investigate soil mobility of rhizosphere volatiles" publiziert: C. Rachow (2018) / Mitt. Dtsch. Ges. Allg. Angew. Ent. 21, S. 313-318 


\subsubsection{Material und Methoden}

\section{Die Apparatur}

Die Apparatur besteht aus einem quadratischen $8 \mathrm{~cm} \mathrm{x} 8 \mathrm{~cm}$ und 1,30 $\mathrm{m}$ langen Aluminium-Gehäuse (Abb. 26). Das Gesamtvolumen der Apparatur beträgt 8,3 1. Beide Enden der Apparatur sind offen und können durch speziell angefertigte Verschlusskappen mit Hebelverschluss an beiden Seiten verschlossen werden. Eine schmale Aluminiumschiene $(110 \mathrm{~cm} \mathrm{x} 3 \mathrm{~cm}$ x 0,5 cm) mit 11 Gewindelöchern von 1,4 cm Durchmesser befindet sich auf der Oberseite des Gehäuses. An jeder Seite der Verschlusskappen befindet sich jeweils ein weiteres Gewindeloch. Die Gewindelöcher auf der Oberseite haben einen Abstand von $10 \mathrm{~cm}$ zueinander und reichen bis ins Innere des Aluminium-Gehäuses. In jedes Gewindeloch kann ein Teflonadapter für die VOC-Beprobung mittels einer SPME-Faser (solid phase microextraction) eingeschraubt werden (Abb. 27). Teflon ist geruchlos und sehr stabil und daher gut als Adaptermaterial geeignet. Dem Anhang Abb. A.3 und Abb. A.4 sind die technischen Zeichnungen, nach der die Apparatur von A. Teichmann gebaut wurde, zu entnehmen.

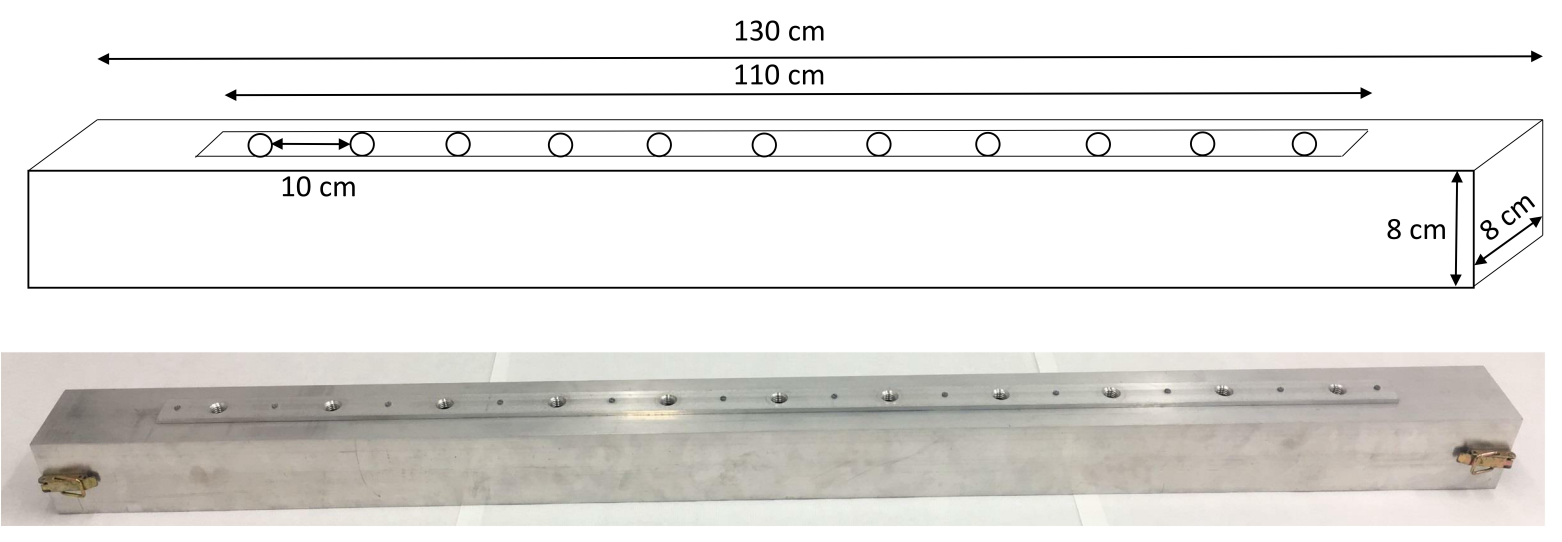

Abbildung 26: Apparatur mit Maßangaben ohne Teflonadapter

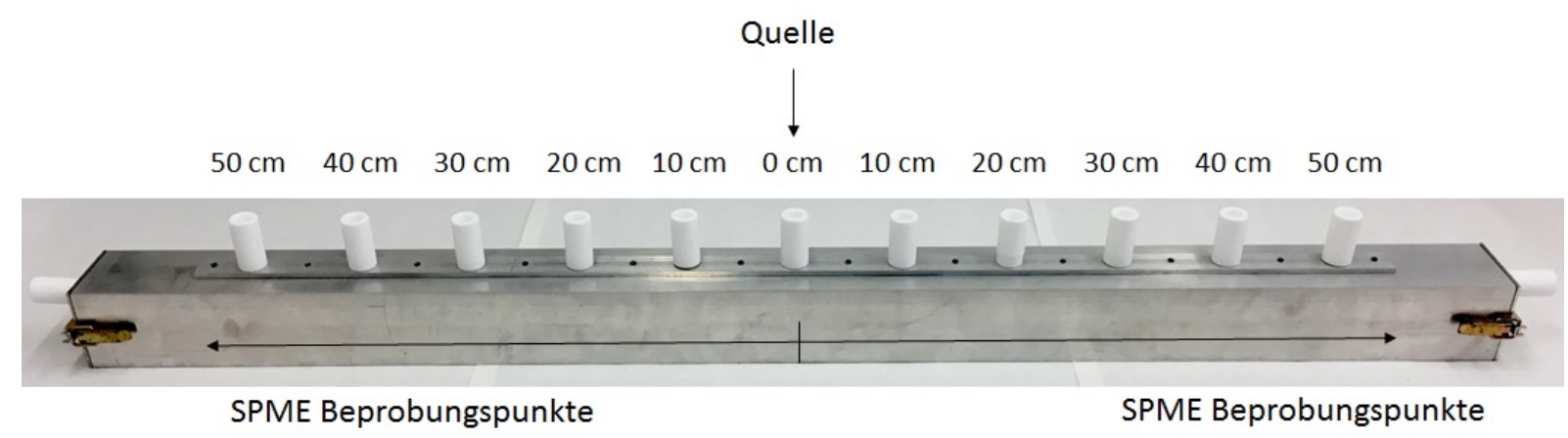

Abbildung 27: Aufbau der Apparatur mit eingedrehten Teflonadaptern und Markierung der unterschiedlichen Probenahmepositionen der SPME-Fasern 
Abb. 28 zeigt die Verschlusskappen der Apparatur. Die Endkappen sind an der Außenseite $8 \mathrm{~cm} \times 8 \mathrm{~cm}$ und an der Innenseite $7,2 \mathrm{~cm}$ x 7,2 $\mathrm{cm}$ breit. Zwischen Außenund Innenrand der Verschlusskappen besteht ein Abstand von 11,5 cm, wodurch sich ein sandfreies Volumen von 0,7 l ergibt (Abb. 28). In die Außenseite jeder Verschlusskappe kann mittels eines Gewindelochs ein Teflonadapter montiert werden. In die Innenseite der Verschlusskappe ist eine feine Metallgaze (14 Maschen/cm) von $4 \mathrm{~cm}$ Durchmesser eingelassen, welche verhindern soll, dass etwas vom Probesubstrat in den Zwischenraum der Verschlusskappen gelangt. Dieser Zwischenraum dient als Messraum für ankommende VOC mittels SPME-Fasern. Abzüglich der Verschlusskappenvolumen ist das Gesamtvolumen der mit Probesubstrat befüllbaren Apparatur 6,9 l.
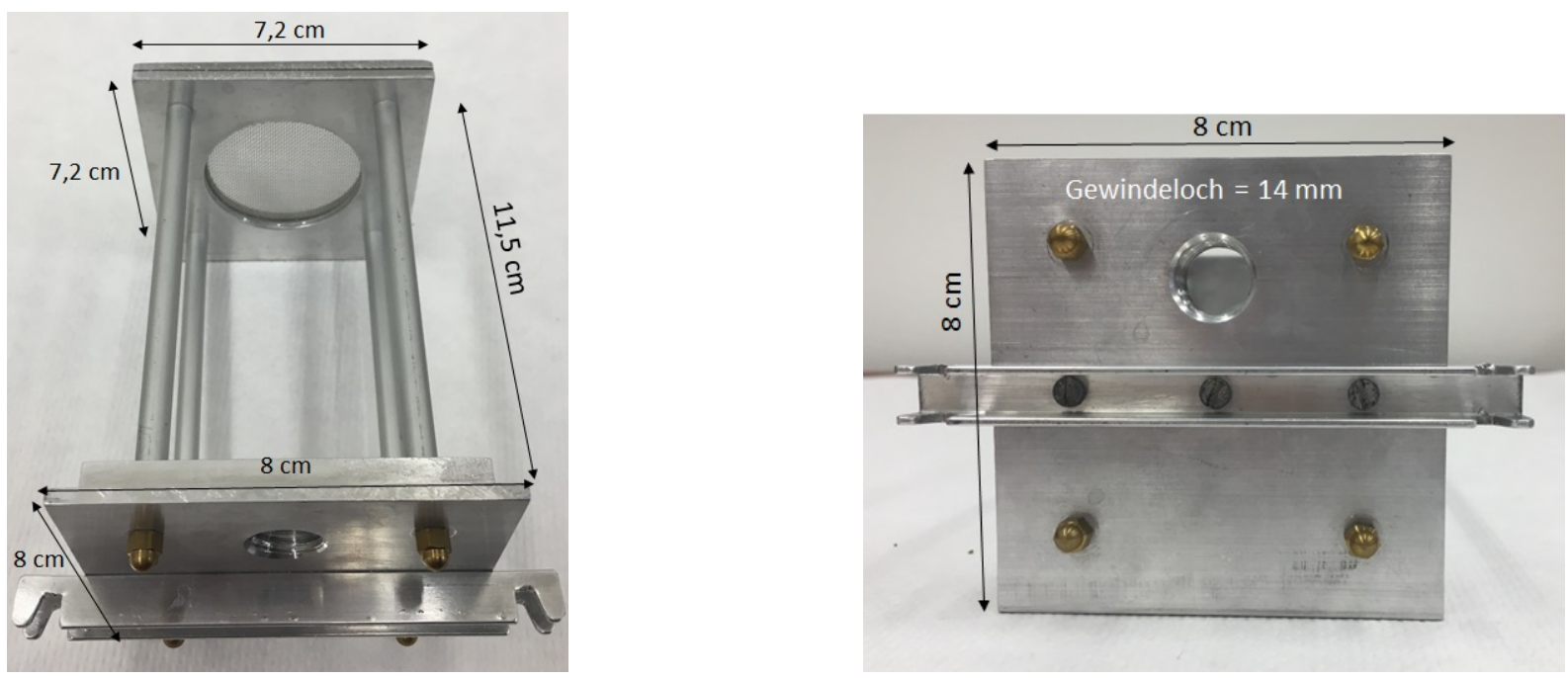

Abbildung 28: Seiten- und Frontansicht der Verschlusskappe

Abb. 29 zeigt einen Teflonadapter mit Metallgehäuse (14 Maschen/cm) am Ende. Das Metallgehäuse ist 6,7 cm lang und schützt die SPME-Faser vor dem Probesubstrat. In jedem Adapter kann eine SPME-Faser durch ein schmales Loch eingelassen werden, welches im Metallgehäuse (von ca. $7 \mathrm{~cm}^{2}$ ) endet (Abb. 30 ).

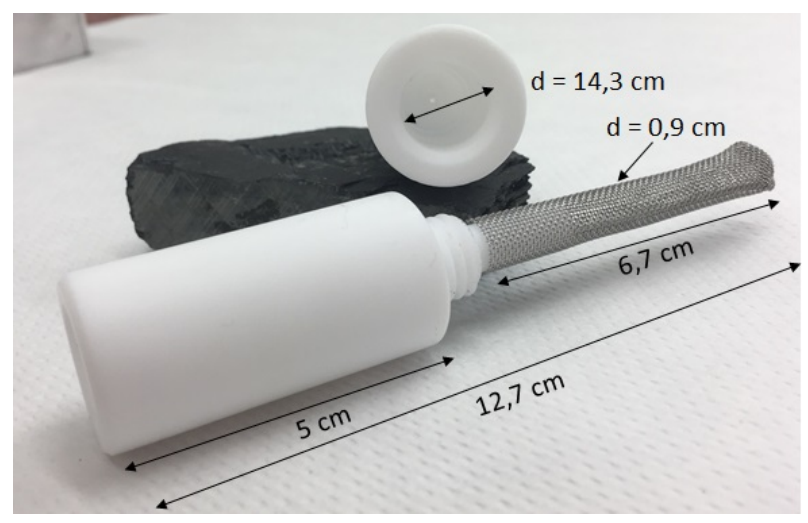

Abbildung 29: Teflonadapter mit Metallgehäuse 


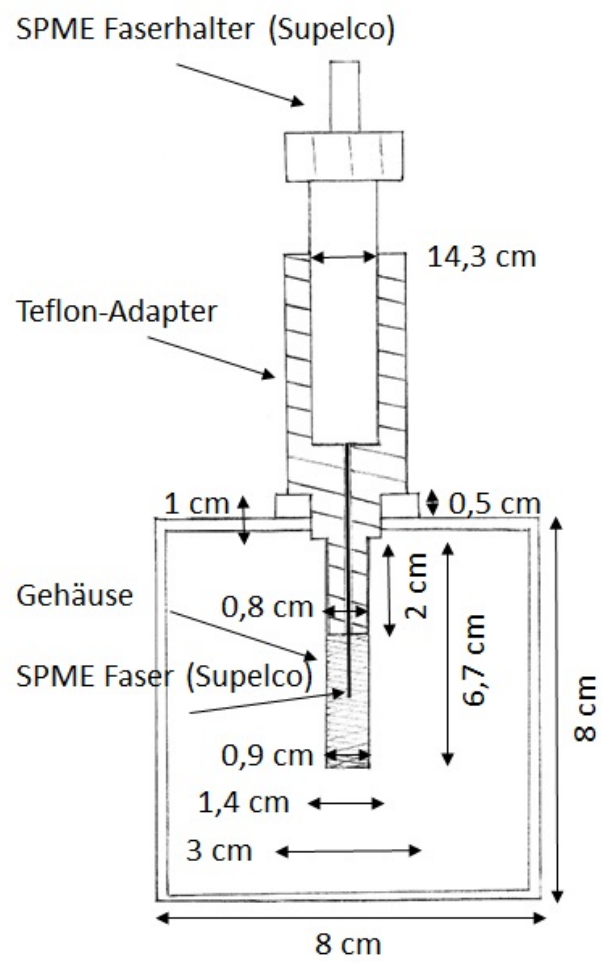

Abbildung 30: Querschnitt der Apparatur

\section{Probesubstrat}

Als Probesubstrat für die Befüllung der Apparatur wurde gereinigter und gewaschener Quarzsand mit einer Gesteinskörnung von 0-2 mm der Firma A\&G-heute verwendet.

Vor der jeweiligen Befüllung der Apparatur mit dem Probesubstrat wurde dieser für zwei Stunden bei $120{ }^{\circ} \mathrm{C}$ ausgeheizt und danach für zwei Stunden abgekühlt. Für jeden Versuch wurde neuer Sand verwendet.

\section{Chemikalien}

Für die Versuche wurden drei VOC verwendet (zwei Ketone: 2-Heptanon, 3-Octanon und ein Alkohol: 3-Octanol). Alle drei VOC besitzen die gleiche relative Dichte $(0,82 \mathrm{~g}$ $\mathrm{cm}^{-3}$ bei $20{ }^{\circ} \mathrm{C}$ ), aber unterschiedliche molare Masse, Dampfdruck und Wasserlöslichkeit (Tab. 12). Die Auswahl der VOC erfolgte aufgrund von Ergebnissen aus den vorherigen Wurzeluntersuchungen (s. Kapitel 4.2.2) und ihrer geringen LD-Einordnung (letale Dosis: $\mathrm{LD}_{50}$ : 2-Heptanon $=10.300 \mathrm{mg} \mathrm{kg}{ }^{-1}$, 3-Octanon und 3-Octanol $>5.000 \mathrm{mg} \mathrm{kg}^{-1}$ ). 
Tabelle 12: Chemische, physikalische und allgemeine Eigenschaften der VOC, die bei der Erprobung der Apparatur verwendet wurden

\begin{tabular}{|l|r|r|r|}
\hline & 2-Heptanon & 3-Octanon & 3-Octanol \\
\hline Summenformel & $\mathrm{C}_{7} \mathrm{H}_{14} \mathrm{O}$ & $\mathrm{C}_{8} \mathrm{H}_{16} \mathrm{O}$ & $\mathrm{C}_{8} \mathrm{H}_{18} \mathrm{O}$ \\
\hline CAS & $110-43-0$ & $106-68-3$ & $589-98-0$ \\
\hline Hersteller (Reinheit 99 \%) & Sigma-Aldrich & Merck & Merck \\
\hline Stoffgruppe & Ketone & Ketone & Alkohol \\
\hline Molare Masse & $114,19 \mathrm{~g} \mathrm{~mol}^{-1}$ & $128,21 \mathrm{~g} \mathrm{~mol}^{-1}$ & $130,23 \mathrm{~g} \mathrm{~mol}^{-1}$ \\
\hline Relative Dichte $\left.\mathbf{( 2 0}^{\circ} \mathbf{C}\right)$ & $0,82 \mathrm{~g} \mathrm{~cm}^{-3}$ & $0,82 \mathrm{~g} \mathrm{~cm}^{-3}$ & $0,82 \mathrm{~g} \mathrm{~cm}^{-3}$ \\
\hline Dampfdruck $\left(\mathbf{2 0}^{\circ} \mathbf{C}\right)$ & $4,5 \mathrm{hPa}$ & $2 \mathrm{hPa}$ & $1,3 \mathrm{hPa}$ \\
\hline Löslichkeit $\left.\left(\mathbf{H}_{2} \mathbf{O}\right) \mathbf{( 2 0}^{\circ} \mathbf{C}\right)$ & $4,3 \mathrm{~g} \mathrm{l}^{-1}$ & $2,6 \mathrm{~g} \mathrm{~cm}^{-3}$ & $1,5 \mathrm{~g} \mathrm{~cm}^{-3}$ \\
\hline Geruch & fruchtig & pilzartig & beißend; mild fruchtig \\
\hline Herkunft & Pilzduft & Pilzduft & Pilzduft \\
\hline
\end{tabular}

\section{Verdünnungsreihen}

Für das Ansetzen der Verdünnungsreihen wurde mit Paraffin (Uvasol, Merck) gearbeitet. Für den ersten Verdünnungsschritt $\left(10^{-1}\right)$ wurden 1,8 g Paraffin und 0,2 g der Reinsubstanz in ein Vial (G4, CS Chromatographie, Langerwehe, Deutschland) mit Schraubverschluss abgewogen und für insgesamt zwei Minuten auf einem Vortex (IKA Vortex 2, Staufen, Deutschland) vermischt. Für die weiteren Verdünnungsschritte $\left(10^{-2}\right.$ bis $\left.10^{-4}\right)$ wurden 1,8 g Paraffin mit 0,2 g von dem vorherigen höheren angefertigten Verdünnungsschritt für jeweils zwei Minuten auf dem Vortex vermischt.

Die fertigen Verdünnungsschritte wurden bis zu ihrer Verwendung in einem Gefrierschrank bei $-20{ }^{\circ} \mathrm{C}$ gelagert. Kurz vor den Versuchen wurde die Verdünnung erneut für eine Minute auf dem Vortex vermischt, damit gewährleistet war, dass eine einheitliche Lösung bei dem jeweiligen Versuch benutzt wurde.

\section{Reinigung der Apparatur}

Vor jedem Versuch wurde die Apparatur gründlich gereinigt. Hierfür wurde der Sand komplett aus der Apparatur entfernt. Die leere Apparatur und die Verschlusskappen wurden für 2,5 Stunden in ein großes Becken mit handwarmem Leitungswasser gelegt. Das Wasser bedeckte die Apparatur dabei vollständig. Zum Wasser wurde noch eine Verschlusskappe voll Reinigungsmittel ( $8 \mathrm{ml}$, RBS Neutral T, ROTH) gegeben. Kurz vor Ablauf der Zeit wurden die einzelnen Bestandteile der Apparatur im Wasser hin und her bewegt, um restliche Sandanhaftungen zu lösen. Anschließend wurden die einzelnen Kompartimente erneut für 1,5 Stunden ins Wasser gelegt, um diese vom überschüssigen Reinigungsmittel zu befreien. Über Nacht wurde die Apparatur zum Trocknen unter eine Abzugshaube gelegt. Kurz vor jedem Versuch wurden sowohl die Außen- als auch die Innenseiten der Apparatur und die Verschlusskappen mit 70 \% Ethanol abgewischt.

Während der Hauptversuche wurde die Apparatur nach Beendigung eines Versuches zu- 
sätzlich mit $5 \mathrm{ml}$ des unpolaren Lösungsmittel Heptan gereinigt, um mögliche Restmengen des unpolaren 3-Octanon zu entfernen.

Die Drahtgehäuse und die Teflonadapter wurden nach jeder Verwendung zweimal in der Spülmaschine mit Reinigungsmittel (RBS Neutral T) bei $55{ }^{\circ} \mathrm{C}$ für 90 min gewaschen und anschließend an der Luft getrocknet.

\section{Faserkalibrierung}

Vor den Versuchen wurde eine einmalige Faserkalibrierung durchgeführt, um mögliche Schwankungen in den Anreicherungseigenschaften zwischen den verwendeten sechs Fasern festzustellen.

Für die Faserkalibrierung wurden zwei Messreihen mit zwei Kalibierungsdurchgängen mit je drei Fasern durchgeführt. Bei jedem Durchgang wurde ein Aluminiumblättchen $(1 \mathrm{~cm} \times 1 \mathrm{~cm})$ mit $50 \mathrm{\mu l}$ einer $10^{-4}$ Verdünnung von 3-Octanon pipettiert, in einem Vial (G1, CS Cromatographie Service, Langerwehe, Deutschland) platziert und mit der jeweiligen SPME-Faser beprobt. Die Beprobungsdauer pro Faser betrug zwei Stunden.

\section{Vorversuche}

In den Vorversuchen wurde untersucht, welche Konzentration des zu untersuchenden VOC für den Hauptversuch geeignet ist und welche Beprobungszeit benötigt wird.

Für die Vorversuche wurde ein einfacher Versuchsaufbau verwendet (Abb. 31). Hierfür wurden Bechergläser (1 l) mit einer $10 \mathrm{~cm}$ dicken Schicht des Probesubstrats befüllt (Abb. 31) und mit Alufolie abgedeckt. Das Probesubstrat wurde bei den Vorversuchen ebenfalls wie zuvor beschrieben behandelt. Anschließend wurde pro Becherglas eine SPME-Faser verwendet und diese mittels Stativ direkt über dem Probesubstrat platziert, ohne das Substrat selbst zu berühren.

Während der ersten Messreihe wurden drei Proben vom Probesubstrat genommen (Tab. 13), um die vom Substrat freigesetzten VOC zu messen. Zusätzlich zur Kontrolle wurden zwei weitere Bechergläser vor der Befüllung präpariert. Dafür wurde ein Filterpapier $(2 \mathrm{~cm} \times 2 \mathrm{~cm})$ mit $2 \mathrm{ml}$ der jeweiligen untersuchten Substanz (2-Heptanon und 3-Octanon) in $10^{-4}$ Verdünnung getränkt und auf den Boden des Becherglases gelegt. Die Durchgänge der ersten Messreihe unterschieden sich jeweils in ihrer Beprobungsdauer.

Die Vorversuche gliedern sich in unterschiedliche Teilexperimente (Menge und Verdünnung der Testsubstanz sowie Beprobungsdauer). Die Details der jeweiligen Messreihe sind in Tab. 13 aufgelistet. Der Aufbau war bei jeder Messreihe identisch mit der ersten Messreihe. 


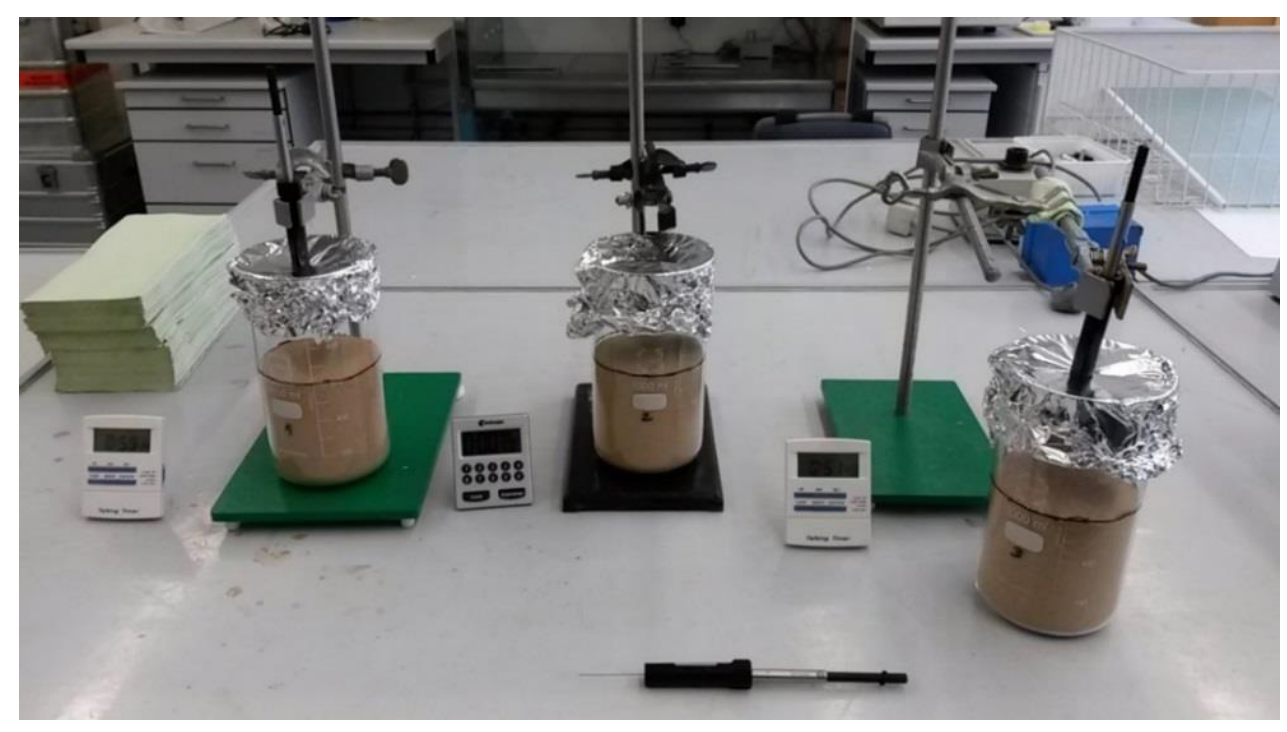

Abbildung 31: Aufbau der Vorversuche (Foto: A. Klamm)

Tabelle 13: Übersicht der Vorversuche

\begin{tabular}{|c|c|c|c|c|c|}
\hline Versuch-Nr. & Testsubstanz & Verdünnung & Menge & Messzeit [min] & Faser-Nr. \\
\hline \multirow[t]{9}{*}{1} & Kontrolle & - & - & $0-60$ & 6 \\
\hline & 2-Heptanon & $10^{-4}$ & $2 \mathrm{ml}$ & $0-60$ & 9 \\
\hline & 3-Octanon & $10^{-4}$ & $2 \mathrm{ml}$ & $0-60$ & 10 \\
\hline & Kontrolle & - & - & $90-150$ & 6 \\
\hline & 2-Heptanon & $10^{-4}$ & $2 \mathrm{ml}$ & $90-150$ & 9 \\
\hline & 3-Octanon & $10^{-4}$ & $2 \mathrm{ml}$ & $90-150$ & 10 \\
\hline & Kontrolle & - & - & $180-240$ & 6 \\
\hline & 2-Heptanon & $10^{-4}$ & $2 \mathrm{ml}$ & $180-240$ & 9 \\
\hline & 3-Octanon & $10^{-4}$ & $2 \mathrm{ml}$ & $180-240$ & 10 \\
\hline \multirow[t]{4}{*}{2} & 2-Heptanon & $10^{-4}$ & $4 \mathrm{ml}$ & $0-120$ & 9 \\
\hline & 3-Octanon & $10^{-4}$ & $4 \mathrm{ml}$ & $0-120$ & 10 \\
\hline & 2-Heptanon & $10^{-4}$ & $4 \mathrm{ml}$ & $150-270$ & 9 \\
\hline & 3-Octanon & $10^{-4}$ & $4 \mathrm{ml}$ & $150-270$ & 10 \\
\hline \multirow[t]{2}{*}{3} & 2-Heptanon & $10^{-4}$ & $2 \mathrm{ml}$ & $0-780$ & 9 \\
\hline & 3-Octanon & $10^{-4}$ & $2 \mathrm{ml}$ & $0-780$ & 10 \\
\hline \multirow[t]{2}{*}{4} & 2-Heptanon & $10^{-4}$ & $10 \mathrm{ml}$ & $0-840$ & 9 \\
\hline & 3-Octanon & $10^{-4}$ & $10 \mathrm{ml}$ & $0-840$ & 10 \\
\hline \multirow[t]{2}{*}{5} & 2-Heptanon & $10^{-2}$ & $10 \mathrm{ml}$ & $0-840$ & 9 \\
\hline & 3-Octanon & $10^{-2}$ & $10 \mathrm{ml}$ & $0-840$ & 10 \\
\hline \multirow[t]{2}{*}{6} & 2-Heptanon & $10^{-2}$ & $10 \mathrm{ml}$ & $0-240$ & 9 \\
\hline & 3-Octanon & $10^{-2}$ & $10 \mathrm{ml}$ & $0-240$ & 10 \\
\hline \multirow[t]{2}{*}{7} & 2-Heptanon & $10^{-3}$ & $10 \mathrm{ml}$ & $0-120$ & 9 \\
\hline & 3-Octanon & $10^{-3}$ & $10 \mathrm{ml}$ & $0-120$ & 10 \\
\hline
\end{tabular}




\section{Hauptversuche}

Der Hauptversuch gliederte sich in zwei Teile. Im ersten Teil wurde untersucht, wie lange die Testsubstanz 3-Octanon in der Apparatur verweilt und ob sich die Bodenbeweglichkeit der Testsubstanz zu den beiden Seiten ausgehend vom Startpunkt „Quelle" gleich verhält.

Im zweiten Teil des Hauptversuches wurden mehrere Wiederholungsversuche mit der Testsubstanz 3-Octanon (6 Wiederholungen) und 3-Octanol (2 Wiederholungen) nach einem vorher festgelegten Zeitplan durchgeführt.

Die Abläufe der jeweiligen Versuche unterschieden sich in der Beprobungszeit und in der Position der SPME-Fasern in der Apparatur. Aufgrund der Ergebnisse und Erfahrungswerte in den Vorversuchen wurde in den Hauptversuchen vorwiegend mit der Testsubstanz 3-Octanon in einer $10^{-2}$-Verdünnung $\mathrm{w} / \mathrm{w}$ (= weight/weight) gearbeitet. Im zweiten Teil des Hauptversuches wurde eine weitere Testsubstanz (3-Octanol, 10-2-Verdünnung $(\mathrm{w} / \mathrm{w}))$ verwendet. Vor jedem Versuch wurde die Apparatur wie vorher beschrieben gereinigt und mit dem kurz zuvor ausgeheizten Probesubstrat befüllt.

Das Befüllen der Apparatur erfolgte zu zweit in senkrechter Position. Für die Befüllung wurde das eine Ende der Aluminiumröhre mit einer der Endkappen verschlossen und in jedes Gewindeloch wurde ein Teflonadapter mit Drahtgehäuse eingelassen, damit kein Sand beim Befüllen aus den Gewindelöchern austreten konnte.

Bevor die Testsubstanz in die Apparatur gegeben wurde, wurden alle verwendeten Teflonadapter mit Parafilm abgedeckt, damit zwischen den einzelnen Messungen keine mit der Testsubstanz angereicherte Luft durch die kleinen Bohrungen in den Adaptern entweichen konnte. Von der zu untersuchenden Testsubstanz wurden $200 \mu \mathrm{l}$ in der Verdünnung $10^{-2}$ auf ein $11 \mathrm{~cm}$ langes und $0,5 \mathrm{~cm}$ breites Filterpapier gegeben, welches zuvor in eine Art Ziehharmonika gefaltet wurde. Das präparierte Filterpapier wurde in ein Drahtgehäuse gegeben. Anschließend wurde das mit Filterpaper befüllte Drahtgehäuse an einen Teflonadapter angebracht, welcher dann in das Gewindeloch samt des Drahtgehäuses bei der Startposition „Quelle“ (0 cm, Abb. 27) eingedreht wurde. Nach Ablauf einer zuvor festgelegten Wartezeit (von zwei Stunden bis zu 40 Tagen) konnte die Probenahme mittels SPME-Fasern an verschiedenen Positionen links und rechts von der Startposition erfolgen.

Teil 1: Im ersten Teil der Hauptversuche wurden Kontrollmessungen der Apparatur im leeren und im befüllten Zustand ohne Testsubstanz durchgeführt. Für die Messungen wurden drei SPME-Fasern verwendet. Beprobt wurde die Startposition „Quelle“ (0 cm), sowie die Gewindelöcher am ganz linken und am ganz rechten Ende der Apparatur $(50 \mathrm{~cm})$. Die Beprobungsdauer für die Kontrollmessungen betrug jeweils zwei Stunden.

Anschließend wurde mit den eigentlichen Messungen begonnen. Hierfür wurde, wie zu- 
vor beschrieben, ein mit der Testsubstanz präpariertes Filterpapier in die Startposition der Apparatur gegeben. Nach Zugabe des Filterpapiers wurden nach einer Wartezeit von zwei Stunden die ersten Messungen $10 \mathrm{~cm}$ links und rechts von der Startposition durchgeführt. Weitere Messungen erfolgten nach 4, 6, 94, 96 und 98 Stunden nach Zugabe des Filterpapieres.

Zur Untersuchung, wie lange und in welcher Menge die Testsubstanz in der Apparatur durch die Beprobung des Probesubstrates mittels SPME-Fasern nachweisbar ist, wurden 120, 146, 256, 276 und 976 Stunden nach Zugabe des mit der Testsubstanz benetzten Filterpapiers von der Startposition ausgehend der rechte Teil der Apparatur an den Positionen 10, 30 und $50 \mathrm{~cm}$ beprobt.

Teil 2: Im zweiten Teil der Hauptversuche wurden zuerst zwei Messungen von 3-Octanon mit $200 \mu 11^{-2}$-Verdünnung (w/w) ohne Sand durchgeführt, um für spätere Berechnungen einen Vergleichswert für die Startmenge an der Startposition „Quelle“ (0 cm) in der Apparatur für die ersten zwei Messstunden zu erhalten. Hierfür wurde in zwei Bechergläser (1 l) je ein $2 \mathrm{~cm}$ x $2 \mathrm{~cm}$ großes und mit $200 \mu \mathrm{l}$ der Testsubstanz präpariertes Filterpapier gelegt. Die Bechergläser wurden mit Alufolie für zwei Stunden abgedeckt und die SPME-Fasern wurden, wie in der eigentlichen Apparatur auch, in einen Teflonadapter mit Drahtgehäuse eingelassen und über die beiden Bechergläser mittels Stativen positioniert. Nach zwei Stunden wurde dann eine Probenahme von insgesamt zwei Minuten durchgeführt.

In der zweiten Hälfte wurden nach einem festgelegten Zeitplan Versuche mit zwei Apparaturen unter einem Laborabzug durchgeführt. Ein Versuch erstreckte sich über fünf Tage. In Abständen von 24 Stunden wurde pro Apparatur eine Messung mit der Probenahmezeit von zwei Stunden durchgeführt. Bei dem 5-Tage-Versuch wurde nur die rechte Seite ausgehend von der Startposition die Bodenbeweglichkeit der Testsubstanz 3-Octanon gemessen. Insgesamt wurden auf diese Weise sechs Messwiederholungen mit der Testsubstanz 3-Octanon durchgeführt. Dabei wurden stets dieselben SPME-Fasern an der gleichen Messposition verwendet.

Während der Versuche wurden fünf Datalogger (DL-120-TH, Voltcraft) zur Aufzeichnung der Temperatur und der Luftfeuchtigkeit unter dem Abzug installiert. Vier Datalogger wurden in jede Ecke des Abzuges gelegt und ein weiterer in die Mitte des Abzuges.

Zwei weitere 5-Tage-Versuche wurden mit der Testsubstanz 3-Octanol mit der gleichen Menge, Verdünnung und den gleichen Probenahmezeiten durchgeführt. Hier wurden beide Seite ausgehend von der Startposition $(0 \mathrm{~cm})$ in einer Distanz von 10, 20 und $30 \mathrm{~cm}$ beprobt (Anhang Tab. A.10 und Tab. A.11). 


\section{Spurenanalytik}

Das Probesubstrat aus den 5-Tage-Versuchen mit der Testsubstanz 3-Octanol wurde im Anschluss an die Versuche spurenanalytisch untersucht. Dies diente der Überprüfung, ob sich die Testsubstanz innerhalb der Versuche an das Probesubstrat anheften konnte und ob die Bodenbeweglichkeit der Testsubstanz durch den Boden unterbunden werden konnte.

Nach Ablauf der beiden 5-Tage-Versuche mit 3-Octanol wurden 20 g Probesubstrat von der Startposition sowie $10 \mathrm{~cm}$ links und $10 \mathrm{~cm}$ rechts davon entnommen. Die Substratproben wurden in ein $100 \mathrm{ml}$ Becherglas mit $25 \mathrm{ml}$ Pentan gegeben und abgedeckt für zwei Stunden stehen gelassen. Nach Ablauf der Wartezeit wurden die Proben in je einen Rundkolben (Schliff NS29) mittels eines Glastrichters und Filterpapier ( $\varnothing 15 \mathrm{~cm}$ ) filtriert. Anschließend wurden die Rundkolben offen unter den Laborabzug gestellt, damit sich das Lösungsmittel Pentan bis auf einen dünnen Film am Boden des Rundkolbens verflüchtigen konnte. Danach wurden die Rundkolben mit jeweils einem Stopfen aus Teflon (NS29/32), der eine Bohrung für die SPME-Faser besitzt, verschlossen. Durch die Bohrung im Stopfen wurde eine SPME-Faser eingelassen und der Inhalt der jeweiligen Rundkolben wurde für zwei Stunden beprobt (Abb. 32). Nach Abschluss der SPME-Messung wurde in jeden Rundkolben 100 pl Dichlormethan/Methanol-Gemisch (3:1, Merck) pipettiert und damit ausgeschwenkt. Dieses Gemisch wurde für weitere GC-Analysen in Spitzbodengläschen (G1s, CS Chromatographie Service, Langerwehe, Deutschland) umgefüllt.

Zusätzlich zur Untersuchung des Probesubstrates auf 3-Octanol wurden die Proben im GC auf das langkettige Alkan Dodecan analysiert. Dodecan ist ein Produkt des zur Verdünnung verwendeten Paraffins. Aufgrund dessen, dass Alkane weniger reaktionsfreudig sind wie Alkohole, kann Dodecan Aufschluss auf mögliche Behinderungen der Bodenbeweglichkeit der Testsubstanz 3-Octanol in der Apparatur geben. 


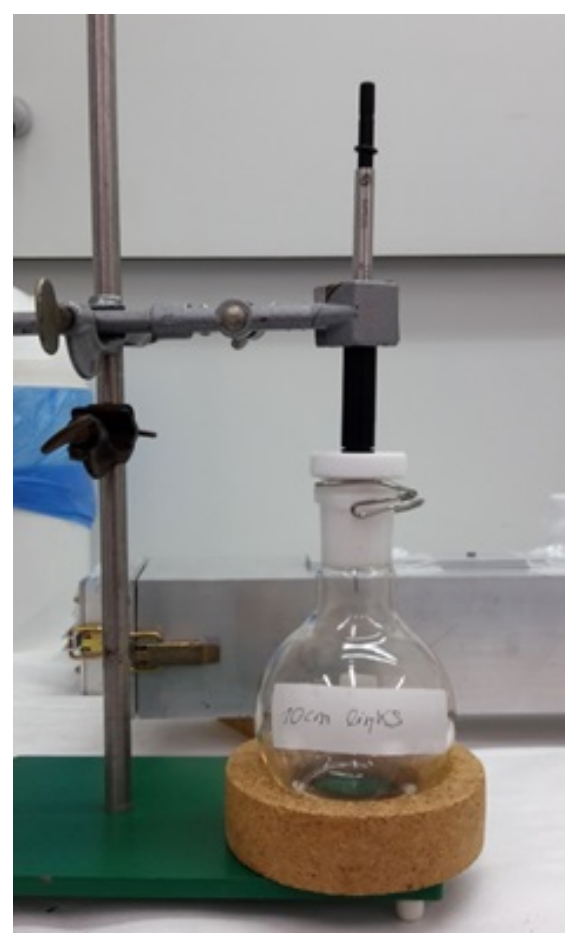

Abbildung 32: SPME-Messung der Substratprobe in Pentan (Foto: A. Klamm)

\section{Verwendete Programme und Software}

Die Datalogger-Daten wurden mit dem Programm USB-Feuchte/Temp.Logger (Version 5.0, Voltcraft) ausgelesen. Je Datalogger wurden Mittelwert und Standardabweichung für die Temperatur- und Luftfeuchtigkeitsdaten berechnet. Aus den Mittelwerten aller fünf verwendeten Datalogger wurden anschließend Mittelwert und Standardabweichung errechnet.

\section{Stoffanalyse und Berechnung der Peakflächen}

Es wurden nur die Proben der Apparatur im leeren Zustand und die des Probesubstrates auf ihr VOC-Muster hin analysiert. Alle anderen Proben wurden nicht auf ihr VOCMuster hin untersucht. Stattdessen wurde gezielt nach der verwendeten Testsubstanz ausgewertet. Für die Analyse der Chromatogramme sowie für die Auswertung einzelner VOC wurde das Programm Enhanced ChemStation Version D 02.00.275 (Agilent Technology) verwendet. Bei der quantitativen Auswertung der einzelnen Testsubstanzen wurde die Peakfläche des Hauptions für die jeweilige Testsubstanz im ,single ion modus“ (SIM) des Auswerteprogrammes Enhanced ChemStatio manuell integiert. Für 2-Heptanon und 3-Octanon wurde die Peakfläche des Ions 43 und für 3-Octanol die des Ions 59 integriert.

Der Mittelwert der beiden Peakflächen aus den Vergleichsmessungen mit 3-Octanon wurde mit 60 multipliziert, um die Messdauer mit der durchgeführten Probenahmezeit von zwei Stunden vergleichbar zu machen. Aufgrund der Anreicherungseigenschaften der 
SPME-Faser (Anreicherung an der Faser ist am Anfang höher als am Ende) wurde der Wert anschließend um den Faktor 4 nach unten korrigiert. Der so ermittelte Vergleichswert diente bei der Auswertung der Hauptversuche als „Startmenge“ an der Startposition zum Zeitpunkt Null $(\mathrm{t}=0)$. Dieser Wert für die „Startmenge“ ist bei der späteren Diffusionsberechnung von Bedeutung.

\section{Diffusion}

Der berechnete Flächeninhalt der Poren unter der Annahme der dichtesten Kugelpackung zur Berechnung des Diffusionskoeffizienten ist ein angenäherter Wert des tatsächlichen Flächeninhaltes der Poren. Die Korngrößen im Probesubstrat variierten zwischen 0 und $2 \mathrm{~mm}$, wodurch sich kleinere Körner in die Zwischenräume größere Poren setzen können. Da das Substrat nicht durch Vibration verdichtet wurde, entspricht die Packung des Substrates in der Apparatur nur annähernd der der Kugelpackung. Die Berechnung des Diffusionskoeffizienten ist somit nur als ungefähre Größenordnung einzuschätzen. Für alle Messwerte wurden die Halbmaxima verwendet, da die Messwerte nicht die tatsächlichen Maxima widerspiegeln und der Fehler somit verringert wurde.

Für die Versuche aus der zweiten Hälfte des zweiten Hauptversuches wurden die Diffusionskoeffizienten für die Messungen mit 3-Octanon berechnet, um die Funktionalität des Versuchsaufbaus zu überprüfen. Dabei wurde die Annahme zugrunde gelegt, dass die Diffusion der einzig vorkommende Transportvorgang während der 5-Tage-Versuche war. Zur Berechnung des Diffusionskoeffinzienten wurde die Formel des 1. Fick'schen Gesetzes angewendet (Gleichung 7).

$$
J=\frac{\Delta N}{\Delta t}=-D * A * \frac{\Delta C}{\Delta x}
$$

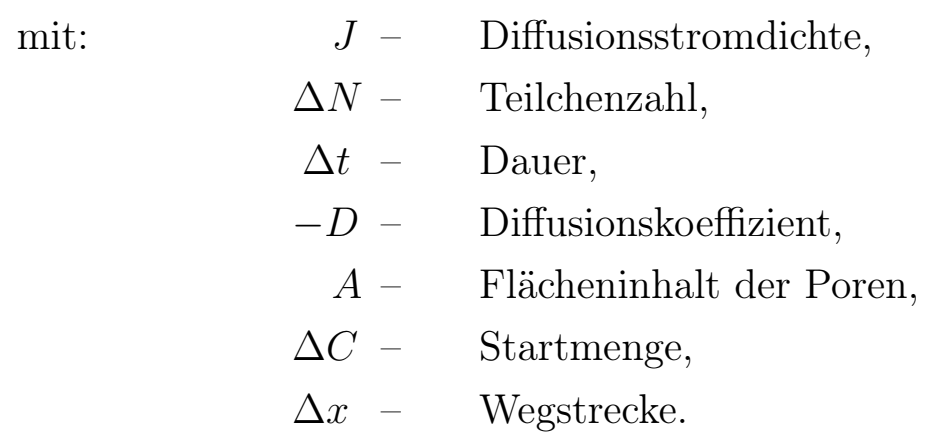

Die Diffusionsstromdichte ergibt sich aus dem Quotienten der Teilchenzahl $\Delta N$ und der Dauer $\Delta t$. Für die Teilchenzahl wurden die jeweiligen Werte der Peakflächen der Halbmaxima aus den Untersuchungen für 10-40 cm (bzw. 10-30 cm) eingesetzt, mit der Annahme, dass die Kalibrierungsgeraden linear verlaufen. Für $\Delta t$ wurde die zweistündige 
Messzeit und für die Startmenge $\Delta C$ die Menge, die bei den Vergleichsmessungen mit 3-Octanon berechnet wurde, verwendet. Die Wegstrecke $\Delta x$ ist die Strecke, für die die Diffusion berechnet werden soll.

Durch Umstellung des 1. Fick'schen Gesetz erhält man den Diffusionskoeffizienten:

$$
-D=\frac{\frac{\Delta N}{\Delta t}}{A * \frac{\Delta C}{\Delta x}}
$$

Bei der Berechnung des Porenvolumens wird die dichteste Kugelpackung mit einer Packungsdichte von $74 \%$ angenommen [259]. Demnach macht das Porenvolumen $26 \%$ vom Gesamtvolumen aus. Um das Porenvolumen für einen Röhrenabschnitt zu berechnen, wurde die Gleichung (9), hier beispielhaft für die ersten $10 \mathrm{~cm}$ der Apparatur vom Startpunkt bis zum Messpunkt $10 \mathrm{~cm}$, angewendet. Breite und Höhe sind $8 \mathrm{~cm}$ und bleiben für alle anderen Messpunkte konstant.

$$
V_{P, 10 \mathrm{~cm}}=l * b * h * k_{\text {Kugelpackung }}
$$

$$
\begin{aligned}
& \text { mit: } \quad V_{P, 10 \mathrm{~cm}}-\quad \text { Porenvolumen für den Messpunkt } 10 \mathrm{~cm} \text {, } \\
& l \text { - Länge }[\mathrm{cm}] \text {, } \\
& b-\text { Breite }[\mathrm{cm}] \text {, } \\
& h \text { - Höhe }[\mathrm{cm}] \text {, } \\
& k_{\text {Kugelpackung }}-\quad \text { Konstante für dichteste Kugelpackung. }
\end{aligned}
$$

Das Porenvolumen wurde benötigt, um den Flächeninhalt der Poren zu berechnen. Hierfür wurde die Gleichung 10 verwendet:

$$
A_{p, 10 \mathrm{~cm}}=\sqrt[3]{V_{P, 10 \mathrm{~cm}}^{2}}
$$

$$
\begin{array}{lll}
\text { mit: } & A_{p, 10 \mathrm{~cm}}- & \text { Flächeninhalt der Poren, } \\
& V_{P, 10 \mathrm{~cm}}- & \text { Porenvolumen für den Messpunkt } 10 \mathrm{~cm} .
\end{array}
$$

Mittels des 2. Fick'chen Gesetzes wurde die Proportionalität zwischen Entfernung und Zeitpunkt des jeweils erreichten Halbmaximums ermittelt $\left(\triangle x^{2} \sim t\right)$. Die sich daraus ergebende Konstante gab Aufschluss über die Diffusionswiderstände entlang des Verlaufes der Apparatur.

Um den theoretischen Zeitpunkt der erreichten Halbmaxima zu ermitteln, wurde zwischen den Messzeitpunkten vor und nach dem jeweiligen Halbmaximum linear mittels 
Trendfunktion in MS Excel 2013 interpoliert. Für die jeweiligen Messzeitpunkte wurde die Mitte der jeweiligen Probenahmezeit verwendet.

\subsubsection{Ergebnisse}

\section{Faserkalibrierung}

Die Ergebnisse der berechneten Peakflächen sind in Tab. 14 dargestellt. Für einen genaueren Vergleich der SPME-Fasern wurden die Peakflächen für das Ion 43 ausgewertet. Die verwendeten Fasern zeigten relativ ähnliche Adsorptionsfähigkeiten zwischen den zwei Kalibrierungsmessungen, unabhängig von der Zwischenlagerungszeit. Allerdings reicherten sich die Fasern im Vergleich untereinander unterschiedlich gut mit der Testsubstanz 3-Octanon an. Nur die beiden Ergebnisse von Faser 10 und 13 liegen nah beieinander. Der größte Wert wurde bei der Faser Nr. 6 und der niedrigste Wert bei Faser Nr. 13 bei der Messreihenfolge 2 berechnet.

Tabelle 14: Peakfläche für das Hauption 43 beider Messreihenfolgen der Faserkalibrierung

\begin{tabular}{|c|r|r|}
\hline Fasernr. & $\begin{array}{r}\text { Peakfläche (in Mio.) Reihenfolge 1: } \\
\mathbf{6 , 9 , 1 0} \text { und 11, 12, 13 }\end{array}$ & $\begin{array}{r}\text { Peakfläche (in Mio.) Reihenfolge 2: } \\
\mathbf{1 0 , 9 , 6} \text { und 13, 12, 11 }\end{array}$ \\
\hline 6 & 60,2 & 73,3 \\
\hline 9 & 53,3 & 47,9 \\
\hline 10 & 46,3 & 46,3 \\
\hline 11 & 45,2 & 32,8 \\
\hline 12 & 35,4 & 38,4 \\
\hline 13 & 31,5 & 31,3 \\
\hline
\end{tabular}

\section{Vorversuche}

Das VOC-Muster vom Probesubstrat gab insgesamt acht VOC ab (Anhang Tab. A.12). Vier Peaks dominierten dabei in allen drei Chromatogrammen der Kontrollmessungen. Abb. 33 zeigt diese vier Peaks in den Chromatogrammen der durchgeführten Kontrollmessungen. Drei der vier Peaks wurden als Camphen (1), Benzen (3) und $\alpha$-Cedren (4) identifiziert. Der Peak Nr. 2 gehört zu Cyclotetrasiloxan, ein VOC, das methodenbedingt aus der Säule des GC stammt. 


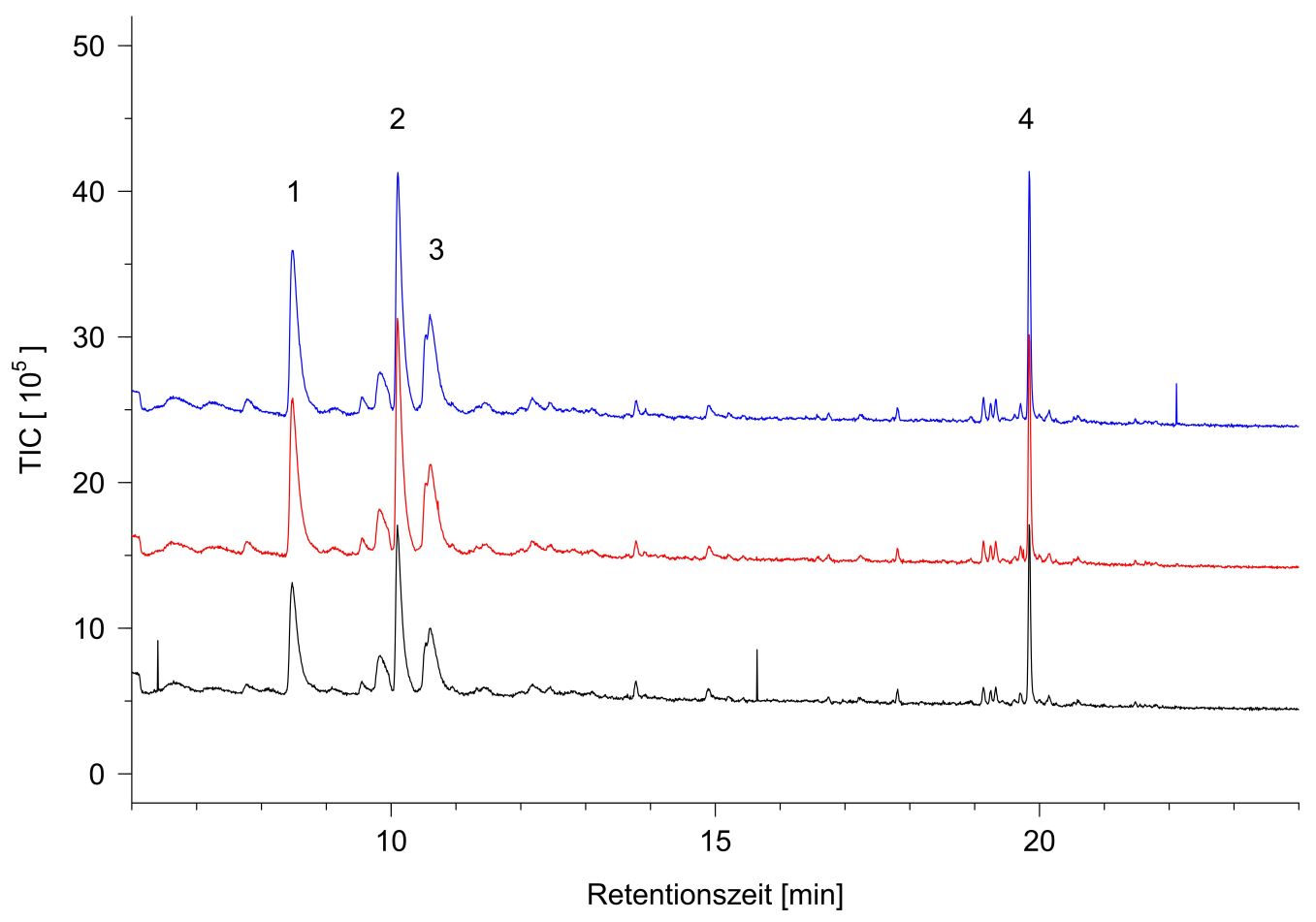

Abbildung 33: Chromatogramme der Kontrollmessungen des Probesubstrates (TIC $=$ Totalionenstrom). Zahlen kennzeichnen die vier Hauptpeaks: $1=$ Camphen, $2=$ Cyclotetrasiloxan, $3=$ Benzen, $4=\alpha$-Cedren

Die Vorversuche zeigten, dass bei einer Konzentration von $10^{-4}(\mathrm{w} / \mathrm{w})$ keine Beweglichkeit der beiden VOC 2-Heptanon und 3-Octanon nach einer Beprobungsdauer von 14 Stunden durch eine $10 \mathrm{~cm}$ hohe Sandschicht nachgewiesen werden konnte. Dagegen konnte bei der Konzentration $10^{-2}(\mathrm{w} / \mathrm{w})$ und einer Menge von $10 \mathrm{ml}$ eine Bewegung der beiden untersuchten VOC durch die Sandschicht nachgewiesen werden. In den darauffolgenden Hauptversuchen wurde auf Grundlage dieses Ergebnisses die Verdünnung $10^{-2}$ (w/w) für die Testsubstanz verwendet.

In Tab. 15 sind die berechneten Peakflächen von 2-Heptanon (Ion 43) und 3-Octanon (Ion 43) für die Proben, in denen im Vorversuch 2-Heptanon und 3-Octanon identifiziert werden konnten, dargestellt.

Tabelle 15: Peakflächen des Hauptions (Ion 43) für 2-Heptanon und 3-Octanon bei den Vorversuchen

\begin{tabular}{|c|l|r|r|r|r|}
\hline Versuchsnr. & VOC & Verdünnung $\left[\frac{g}{g}\right]$ & Menge & Messzeit [min] & Peakfläche (in Mio.) \\
\hline \multirow{2}{*}{5} & 2 -Heptanon & $10^{-2}$ & $10 \mathrm{ml}$ & $0-840$ & 901,6 \\
\cline { 2 - 6 } & 3-Octanon & $10^{-2}$ & $10 \mathrm{ml}$ & $0-840$ & 22,8 \\
\hline \multirow{2}{*}{6} & 2 -Heptanon & $10^{-2}$ & $10 \mathrm{ml}$ & $0-240$ & 732,1 \\
\cline { 2 - 6 } & 3 -Octanon & $10^{-2}$ & $10 \mathrm{ml}$ & $0-240$ & 2,9 \\
\hline 7 & 2 -Heptanon & $10^{-3}$ & $10 \mathrm{ml}$ & $0-120$ & 3,2 \\
\hline
\end{tabular}




\section{Hauptversuche}

Teil 1: In den Kontrollmessungen der Apparatur im leeren Zustand konnte bei allen drei Proben Heptanal, Octanal und Nonanal identifiziert werden. In jeweils nur einer Probe wurde Benzeneethanamin und Pentasiloxan gefunden (Anhang Tab. A.13). Im befüllten Zustand mit dem Probesubstrat konnte in den Proben vorwiegend Nonanal und Octanal gemessen werden (Anhang Tab. A.14). Es konnten in keiner der Proben die Testsubstanzen identifiziert werden.

Des Weiteren wurde untersucht, wie sich das VOC 3-Octanon über die ersten $10 \mathrm{~cm}$ links und rechts, ausgehend von der Startposition der Apparatur, ausbreitet. Die ermittelten Peakflächen, die zu den unterschiedlichen Messzeitpunkten in $10 \mathrm{~cm}$ Entfernung von der Startposition gemessen wurden, sind in der Abb. 34 abgebildet. Die dazugehörige Wertetabelle ist dem Anhang (Tab. A.15) zu entnehmen. Bei Betrachtung der Abb. 34 ist zu erkennen, dass beide Kurven einen ähnlichen Verlauf zeigen, die Werte jedoch verzögert sind. $10 \mathrm{~cm}$ rechts von der Duftquelle konnte bereits zum Messzeitpunkt 6-8 Stunden 3-Octanon festgestellt werden. Bei dem Messpunkt $10 \mathrm{~cm}$ links von der Duftquelle konnte erst 94-96 Stunden nach Versuchsbeginn 3-Octanon gemessen werden. Die Werte für $10 \mathrm{~cm}$ links von der Duftquelle liegen stets unter den Werten für $10 \mathrm{~cm}$ rechts von der Duftquelle. Beide Kurven fallen nach dem Messzeitpunkt 96-98 Stunden leicht ab und steigen danach steil an.

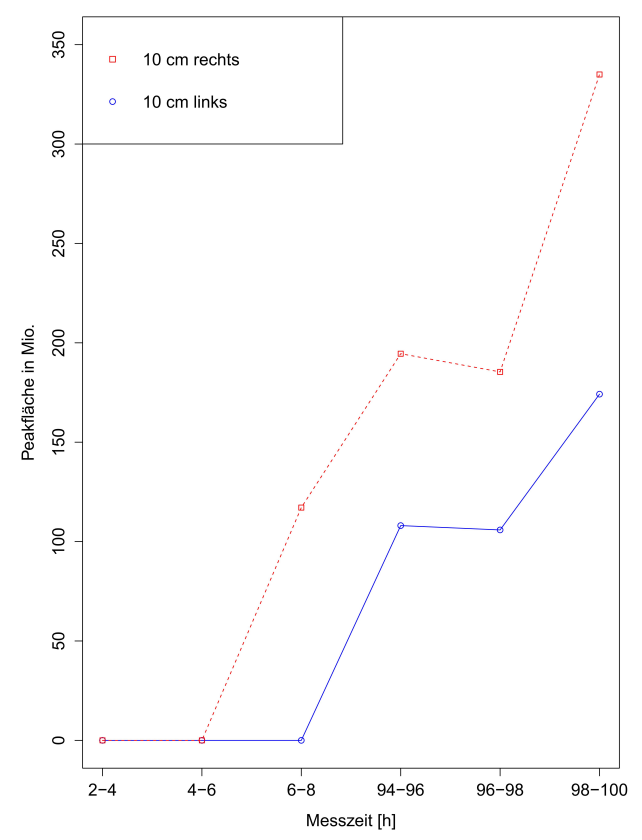

Abbildung 34: Nachweis von 3-Octanon in der Apparatur $10 \mathrm{~cm}$ links und rechts von der Duftquelle zu unterschiedlichen Messzeiten. Dargestellt ist die Peakfläche für das Ion 43. 
Abb. 35 zeigt den Verlauf der Messungen im rechten Arm der Apparatur in 10, 30 und $50 \mathrm{~cm}$ Entfernung zur Duftquelle bei einer Versuchsdauer von 120-978 Stunden. Die dazugehörigen Daten sind dem Anhang (Tab. A.16) zu entnehmen. An allen Messpunkten konnte 3-Octanon gemessen werden, allerdings nicht zu allen Messzeitpunkten. Alle drei Messpositionen hatten zu unterschiedlichen Zeiten ihr Peakflächen-Maximum. Bei der Position $10 \mathrm{~cm}$ konnte das Maximum (165 Mio.) nach einer Versuchsdauer von 120 Stunden festgestellt werden. Bei der Distanz von $50 \mathrm{~cm}$ lag die Peakfläche lediglich bei 567 Tsd. Dieses Größenverhältnis tauscht sich beim zweiten Messzeitpunkt (146-148 Stunden). Hier liegt der Wert der Peakfläche für die Distanz $10 \mathrm{~cm}$ bei 293 Tsd., wogegen bei der Distanz von $50 \mathrm{~cm}$ das Peakflächen-Maximum von 171 Mio. erreicht wurde. Das Maximum (64 Mio.) für die Distanz von $30 \mathrm{~cm}$ konnte nach einer Versuchsdauer von 256-258 Stunden bestimmt werden.

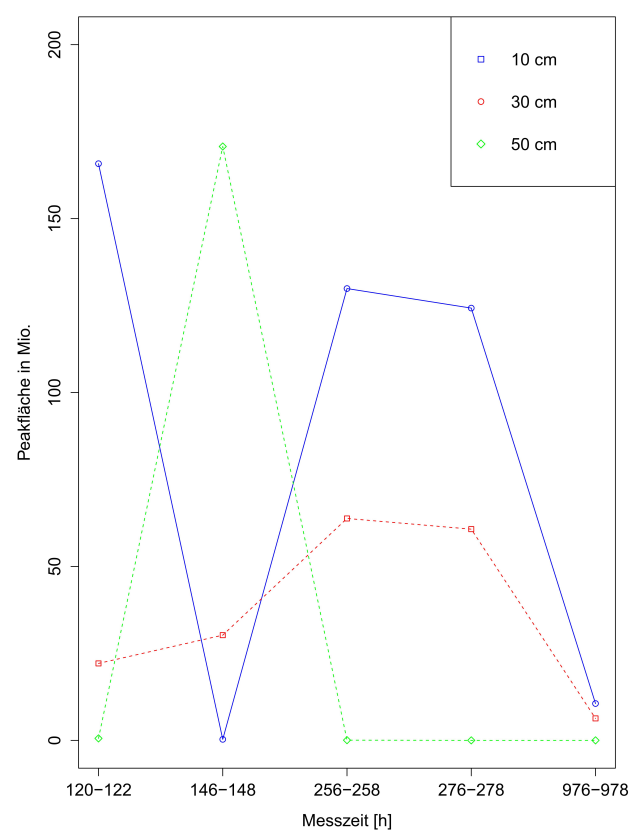

Abbildung 35: Nachweis von 3-Octanon in der Apparatur in 10, 30 und $50 \mathrm{~cm}$ Distanz zur Duftquelle zu unterschiedlichen Zeiten. Dargestellt ist die Peakfläche für das Ion 43.

Teil 2: Die Ergebnisse der Vergleichsmessung für 3-Octanon mit $200 \mu l$ in der Verdünnung $10^{-2}(\mathrm{w} / \mathrm{w})$ sind in Tab. 16 aufgeführt.

Tabelle 16: Vergleichswert für die Startmenge an der Startposition zum Zeitpunkt t $=0$

\begin{tabular}{|c|r|c|}
\hline Messzeit [min] & Peakfläche (Ion 43) & SPME-Faser Nr. \\
\hline 2 & 183.181 .460 & 10 \\
\hline 2 & 115.975 .554 & 13 \\
\hline
\end{tabular}


Aus den zwei Messwerten wurde der Mittelwert (149.578.507) gebildet und mit 60 multipliziert, um einen Vergleichswert für eine zweistündige Messdauer zu erhalten. Dieser Wert wurde dann durch vier dividiert, um die Anreicherungseigenschaften der SPMEFasern zu berücksichtigen. Der so erhaltene Vergleichswert von 2,24 Mrd. wurde bei der Diffusionsberechnung verwendet. Des Weiteren wurden die Messergebnisse der Wiederholungsmessungen im zweiten Teil des Hauptversuches in Relation zum Vergleichswert gesetzt.

3-Octanon konnte nur in den ersten zwei Wiederholungsmessungen bei verschiedenen Distanzen von der Startposition nachgewiesen werden (Abb. 36 und 37). Die Daten der beiden Messungen sind im Anhang (Tab. A.17) einzusehen.

In der ersten Messung konnten 3-Octanon noch nach 98 Stunden in einer Distanz von bis zu $30 \mathrm{~cm}$ von der Startposition entfernt nachgewiesen werden (0,16\% vom Vergleichswert, Abb. 36). Bei der Distanz 10 und $20 \mathrm{~cm}$ wird das Maximum der Peakfläche bei der Messzeit 48-50 Stunden erreicht. Bei beiden Distanzen konnte schon zum ersten Messzeitpunkt 3-Octanon gemessen werden. Bei der Distanz von $30 \mathrm{~cm}$ konnte erst nach 48-50 Stunden 3-Octanon in den Proben gefunden werden. Von dort an nimmt die gemessene Peakfläche bei der Distanz von $30 \mathrm{~cm}$ kontinuierlich zu. Maximal wurden bei der ersten Messung nur $0,24 \%$ vom Vergleichswert in den Proben gefunden.

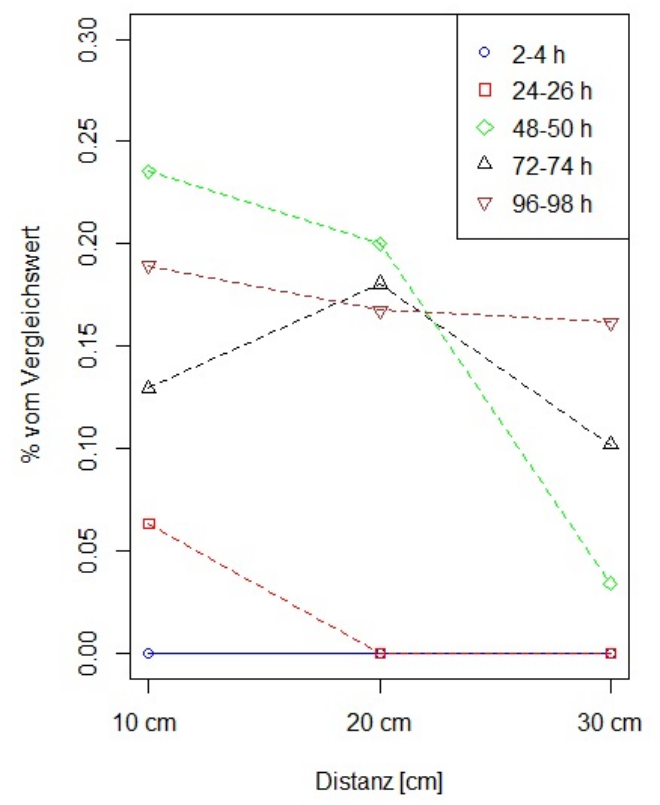

Abbildung 36: 1. Wiederholungsmessung mit 3-Octanon $\left(200 \mu l, 10^{-2}(\mathrm{w} / \mathrm{w})\right)$ in der Apparatur. Dargestellt sind die Peakflächen (Ion 43) bei den jeweiligen gemessenen Distanzen zur Duftquelle bei den unterschiedlichen Messzeitpunkten in \% zum Vergleichswert. 
Bei der zweiten Messung konnte bis zu $14 \%$ vom Vergleichswert in den Proben gefunden werden. Nach zwei Stunden erreichten $7 \%$ vom Vergleichswert eine Distanz von $10 \mathrm{~cm}$, nach 24 Stunden erreichten $11 \%$ eine Distanz von $20 \mathrm{~cm}$, nach 48 Stunden erreichten $3 \%$ eine Distanz von $30 \mathrm{~cm}$ und nach 72 Stunden erreichten 0,07\% des Vergleichswertes eine Distanz von $40 \mathrm{~cm}$ (Abb. 37). Es konnte somit deutlich mehr 3-Octanon in den Proben bei der zweiten Messung als bei der ersten Messung nachgewiesen werden. Im Gegensatz zu der ersten Messung wurde bei den Distanzen 10, 20 und $30 \mathrm{~cm}$ schon bei der ersten Messzeit (2-4 Stunden) 3-Octanon nachgewiesen und bei den Distanzen von $10 \mathrm{~cm}$ und $20 \mathrm{~cm}$ wurde das Peakflächen-Maximum schon nach 24-26 Stunden erreicht. Bei der Distanz von $30 \mathrm{~cm}$ stieg die Peakfläche wie bei der ersten Messung kontinuierlich an. Nach 98 Stunden konnte allerdings auch hier kein 3-Octanon in einer Distanz von $50 \mathrm{~cm}$ zur Startposition nachgewiesen werden.

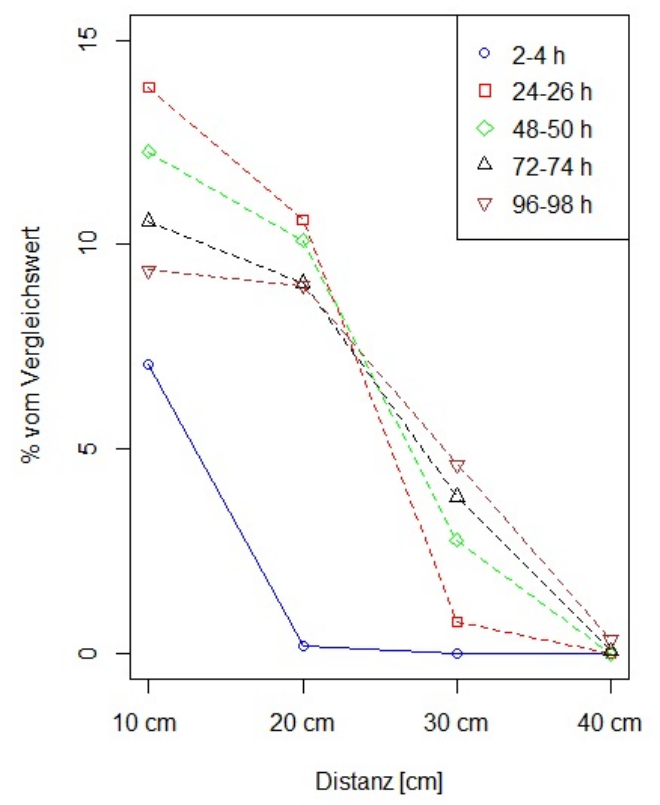

Abbildung 37: 2. Wiederholungsmessung mit 3-Octanon $\left(200 \mu l, 10^{-2}(\mathrm{w} / \mathrm{w})\right)$ in der Apparatur. Dargestellt sind die Peakflächen (Ion 43) bei den jeweiligen gemessenen Distanzen zur Duftquelle bei den unterschiedlichen Messzeitpunkten in \% zum Vergleichswert.

Ab der dritten Wiederholungsmessung konnte kein 3-Octanon in den Proben identifiziert werden. Ebenso konnten in den beiden Untersuchungen zur Bodenbeweglichkeit von 3-Octanol kein 3-Octanol in Proben in 10-30 cm Distanz zur Startposition nachgewiesen werden.

Die Temperatur während der Wiederholungsmessung mit 3-Octanon betrug zwischen 
21,0 und $23,4{ }^{\circ} \mathrm{C}$ und die relative Lufteuchtigkeit zwischen 50,1 und $73,5 \%$.

\section{Diffusion}

In Tab. 17 und 18 sind die Halbmaxima und die durch Interpolation bestimmten Messzeitpunkte für die Messwiederholungen mit 3-Octanon im zweiten Teil des Hauptversuches aufgeführt.

Tabelle 17: Halbmaxima und die dazugehörigen interpolierten Zeitpunkte für die erste Wiederholungsmessung im Hauptversuch mit 3-Octanon. Peakfläche ermittelt im SIM für 3-Octanon (Ion 43)

\begin{tabular}{|c|r|r|r|r|r|r|r|}
\hline & Max. & $\begin{array}{r}\text { Halbmax. } \\
\text { Peakfläche }\end{array}$ & $\begin{array}{r}\text { Peakfäche } \\
\text { vor }\end{array}$ & $\begin{array}{r}\text { Zeitpunkt des } \\
\text { Wertes vor } \\
\text { Halbmax. [h] }\end{array}$ & $\begin{array}{r}\text { Peakfläche } \\
\text { nach } \\
\text { Halbmax. }\end{array}$ & $\begin{array}{r}\text { Zeitpunkt des } \\
\text { Wertes nach } \\
\text { Halbmax. [h] }\end{array}$ & $\begin{array}{r}\text { Interpolierter } \\
\text { Zeitpunkt des } \\
\text { Halbmax. [h] }\end{array}$ \\
\hline $10 \mathrm{~cm}$ & 5.285 .797 & 2.642 .899 & 1.428 .069 & 25 & 5.285 .797 & 49 & 33 \\
\hline $20 \mathrm{~cm}$ & 4.490 .174 & 2.245 .087 & 0 & 25 & 4.490 .174 & 49 & 73 \\
\hline $30 \mathrm{~cm}$ & 3.631 .023 & 1.815 .512 & 764.517 & 49 & 2.290 .816 & 66 \\
\hline
\end{tabular}

Tabelle 18: Halbmaxima und die dazugehörigen interpolierten Zeitpunkte für die zweite Wiederholungsmessung im Hauptversuch mit 3-Octanon. Peakfläche ermittelt im SIM für 3-Octanon (Ion 43)

\begin{tabular}{|c|c|c|c|c|c|c|c|}
\hline Distanz & $\begin{array}{r}\text { Max. } \\
\text { Peakfläche }\end{array}$ & $\begin{array}{l}\text { Halbmax. } \\
\text { Peakfläche }\end{array}$ & $\begin{array}{r}\text { Peakfläche } \\
\text { vor } \\
\text { Halbmax. }\end{array}$ & $\begin{array}{r}\text { Zeitpunkt des } \\
\text { Wertes vor } \\
\text { Halbmax. [h] }\end{array}$ & $\begin{array}{r}\text { Peakfläche } \\
\text { nach } \\
\text { Halbmax. }\end{array}$ & $\begin{array}{r}\text { Zeitpunkt des } \\
\text { Wertes nach } \\
\text { Halbmax. [h] }\end{array}$ & $\begin{array}{l}\text { Interpolierter } \\
\text { Zeitpunkt des } \\
\text { Halbmax. [h] }\end{array}$ \\
\hline $10 \mathrm{~cm}$ & 310.385 .775 & 155.192 .888 & 155.102 .827 & 3 & 310.385 .775 & 25 & 3 \\
\hline $20 \mathrm{~cm}$ & 237.801 .304 & 118.900 .652 & 4.182 .849 & 3 & 237.801 .304 & 25 & 14 \\
\hline $30 \mathrm{~cm}$ & 103.891 .912 & 51.945 .956 & 17.618 .36 & 25 & 62.425 .960 & 49 & 45 \\
\hline $40 \mathrm{~cm}$ & 7.674 .949 & 3.837 .474 & 1.560 .029 & 73 & 7.674 .949 & 97 & 82 \\
\hline
\end{tabular}

In Tab. 19 sind die Diffusionskoeffizienten und in Tab. 20 sind die Ergebnisse der Diffusionskonstante für alle Distanzen beider Wiederholungsmessungen aufgeführt.

Tabelle 19: Diffusionskoeffizienten für beide Wiederholungsmessungen mit 3-Octanon

\begin{tabular}{|c|c|r|}
\hline \multicolumn{2}{|c|}{ Distanz $[\mathrm{cm}]$} & Diffusionskoeffizient $\left[\mathrm{cm}^{2} s^{-1}\right]$ \\
\hline \multirow{5}{*}{ 1. Wiederholung } & 10 & $5,41 * 10^{-8}$ \\
\cline { 2 - 3 } & 20 & $5,79 * 10^{-8}$ \\
\cline { 2 - 3 } & 30 & $5,36 * 10^{-8}$ \\
\hline \multirow{5}{*}{ 2. Wiederholung } & 10 & $3,18 * 10^{-6}$ \\
\cline { 2 - 3 } & 20 & $3,07 * 10^{-6}$ \\
\cline { 2 - 3 } & 30 & $1,53 * 10^{-6}$ \\
\cline { 2 - 3 } & 40 & $1,25 * 10^{-7}$ \\
\hline
\end{tabular}


Tabelle 20: Diffusionskonstante für beide Wiederholungsmessungen mit 3-Octanon

\begin{tabular}{|l|l|r|}
\hline \multirow{2}{*}{ 1. Wiederholung } & $10 \mathrm{~cm}$ & 3,0 \\
\cline { 2 - 3 } & $20 \mathrm{~cm}$ & 10,8 \\
\cline { 2 - 3 } & $30 \mathrm{~cm}$ & 13,6 \\
\hline \multirow{2}{*}{ 2. Wiederholung } & $10 \mathrm{~cm}$ & 33,3 \\
\cline { 2 - 3 } & $20 \mathrm{~cm}$ & 28,6 \\
\cline { 2 - 3 } & $30 \mathrm{~cm}$ & 20,0 \\
\cline { 2 - 3 } & $40 \mathrm{~cm}$ & 19,5 \\
\hline
\end{tabular}

\section{Spurenanalytik}

Im ersten Teil der Spurenanalytik wurden die zwei Metallgehäuse aus dem zweiten Hauptversuch mit 3-Octanol, in denen sich das mit der Probesubstanz benetzte Filterpapier befand, zwei Stunden mittels SPME-Fasern beprobt. In beiden Metallgehäusen konnte 3-Octanol nachgewiesen werden (Peakfläche für Ion 59: 32.363.566 und 16.993.273).

Bei den SPME-Proben des Sand/Pentan-Gemisches konnte nur bei der Startposition 3-Octanol nachgewiesen werden (Tab. 21).

Tabelle 21: SPME-Proben des Sand/Pentan-Gemisches

\begin{tabular}{|c|l|c|r|}
\hline Apparatur & Probe & 3-Octanal identifiziert & Peakfläche (Ion 59) \\
\hline 1 & links $10 \mathrm{~cm}$ & nein & 0 \\
\hline 1 & Mitte $0 \mathrm{~cm}$ & ja & 0 \\
\hline 1 & rechts $10 \mathrm{~cm}$ & nein & 0 \\
\hline 2 & links $10 \mathrm{~cm}$ & nein & 0 \\
\hline 2 & Mitte $0 \mathrm{~cm}$ & nein & 0 \\
\hline 2 & rechts $10 \mathrm{~cm}$ & nein & \\
\hline
\end{tabular}

In Tab. 22 sind die Ergebnisse der Dichlormethan/Methanol-Proben des Sand/PentanGemisches für beide Apparaturen aufgeführt. 3-Octanol konnte in beiden Sandproben aus der Mitte beider Apparaturen identifiziert werden.

Tabelle 22: Dichlormethan/Methanol-Proben des Sand/Pentan-Gemisches

\begin{tabular}{|c|l|c|r|}
\hline Apparatur & Probe & 3-Octanal identifiziert & Peakfläche (Ion 59) \\
\hline 1 & links $10 \mathrm{~cm}$ & nein & 0 \\
\hline 1 & Mitte $0 \mathrm{~cm}$ & ja & 50.459 \\
\hline 1 & rechts $10 \mathrm{~cm}$ & nein & 0 \\
\hline 2 & links $10 \mathrm{~cm}$ & nein & 09.669 \\
\hline 2 & Mitte $0 \mathrm{~cm}$ & ja & 0 \\
\hline 2 & rechts $10 \mathrm{~cm}$ & nein & \\
\hline
\end{tabular}

Das Alkan Dodecan konnte in keiner der Proben, die während der Spurenanalytik genommen wurden, nachgewiesen werden. 


\subsubsection{Diskussion}

Da es noch kein standardisiertes Verfahren zur Messung der Bodenbeweglichkeit von VOC gab, wurde im Rahmen dieser Arbeit eine Apparatur entwickelt, die es ermöglicht, die horizontale Ausbreitung von Wurzel-VOC in einem einheitlichen Probesubstrat zu untersuchen. Die Entwicklung der Apparatur baute dabei auf mehreren kleineren Vorversuche auf. Die Vorteile der Apparatur liegen im einfachen Design und in der Handhabung, da diese, abgesehen von der Befüllung (Gewicht der Apparatur mit Probesubstrat liegt bei $14 \mathrm{~kg}$ ), von einer Person bedient werden kann.

Das verwendete Probesubstrat selbst emittierte drei VOC (Camphen, Benzen und $\alpha$-Cedren). Camphen und Benzen könnten auf chemische Bearbeitungsprozesse wie z. B. Lagerung zurückzuführen sein. Benzen entsteht beispielsweise bei der Zersetzung von organischem Material unter Luftabschluss beziehungsweise bei der Verbrennung von organischem Material [260]. Durch die Trocknung des Probesubstrates bei $120{ }^{\circ} \mathrm{C}$ könnte aus organischem Material, welches möglicherweise im Substrat vorhanden war, Benzen entstanden sein. Das ST $\alpha$-Cedren ist ein Isomer von Cedren, das von verschiedenen Koniferenarten emittiert wird 261,262]. Eventuell wurde das Probesubstrat zuvor in Holzkisten gelagert und hat sich dadurch mit Cedren angereichert. Im leeren Zustand konnten im VOC-Muster der Apparatur die Aldehyde Heptanal, Octanal und Nonanal nachgewiesenen werden. Das Vorkommen dieser VOC ist möglicherweise auf die Reinigung der Apparatur mit Ethanol zurückzuführen, da bei der Oxidation von primären Alkoholen Aldehyde entstehen können [263]. Die Testsubstanzen 3-Octanon und 3-Octanol konnten allerdings weder im leeren noch im befüllten Zustand nachgewiesen werden.

Im ersten Teil des Hauptversuches konnte 3-Octanon zuerst rechts neben der Quelle detektiert werden. Die berechneten Peakflächen der Proben, die rechts neben der Quelle entnommen wurden, waren teilweise doppelt so hoch wie die der Proben, die links von der Startposition entnommen wurden (Anhang Tab. A.15). Eine möglicher Grund dafür könnte die Befülltechnik der Apparatur mit dem Probesubstrat sein. Die Apparatur wurde aufrecht befüllt. Dabei war die linke Seite der Apparatur immer abwärts gerichtet, was dazu geführt haben könnte, dass das Substart in der linken Seite dichter gepackt wurde als in der rechten Seite der Apparatur. Die möglicherweise dichtere Packung des Probesubstrates in der linken Seite könnte verursacht haben, dass 3-Octanon weniger schnell durch das Substrat diffundiert ist. Dies könnte die größeren Peakflächen erklären und dass 3-Octanon in der rechten Apparaturseite in $10 \mathrm{~cm}$ Entfernung zur Startposition zuerst detektiert wurde.

Bei den Hauptversuchen konnte nur in den ersten beiden Wiederholungsmessungen 3-Octanon detektiert werden. Trotz gleicher Ausgangssituation und paralleler Messung wichen die Ergebnisse der ersten beiden Wiederholungsmessungen voneinander ab. Für 
alle weiteren Wiederholungsmessungen konnte kein 3-Octanon in der Apparatur nachgewiesen werden. Eine mögliche Ursache könnte ein vorherrschender Senkeneffekt innerhalb der Apparatur durch die Ansammlung der Testsubstanz an der Oberfläche der Apparatur aufgrund von z. B. unzureichender Durchmischung des Probesubstrates sein. Dies könnte auch zu einer Verzögerung beim Anstieg bzw. Abfall der Substanzkonzentration führen 264. Eine weitere Ursache könnte die Temperatur sein. Die Diffusion, und somit die Bodenbeweglichkeit der VOC, wird von der Temperatur beeinflusst 265. Während der sechs Wiederholungsmessungen lagen die Mittelwerte für die Temperatur zwischen 21,0 und $23,4{ }^{\circ} \mathrm{C}$. Da nicht ausgeschlossen werden kann, dass bereits geringe Temperaturunterschiede einen Einfluss auf die Molekülbewegung der Test-VOC hatten, sollten weitere Untersuchungen in einer Klimakammer durchgeführt werden, in der die Temperatur und Luftfeuchtigkeit gesteuert werden können.

In der Apparatur konnte der Stofftransport, anders als im Boden, nicht ungehindert dreidimensional stattfinden. Der Stofffluss war durch die Höhe und Breite der Apparatur beschränkt und konnte nur horizontal ungehindert ablaufen. Dadurch kann es sein, dass der Konzentrationsgradient durch die Barrieren unnatürlich lange hochgehalten wurde. Im Anfangsbereich höhere Konzentrationen, bedingt durch ein Ansammeln der Testsubstanz an den Wandbarrieren, könnte für ein höheres Konzentrationsgefälle in der Längsrichtung gesorgt haben. Dies könnte dazu geführt haben, dass der gemessene Stofffluss höher ausgefallen ist, als dies unter natürlichen, dreidimensionalen Bedingungen der Fall gewesen wäre. Es kann ebenfalls nicht ausgeschlossen werden, dass sich aufgrund der Befülltechnik Hohlräume an der Decke der Apparatur gebildet haben. Dadurch, dass nach der Befüllung nicht weiter nachverdichtet wurde, könnte sich nach dem Ablegen der Apparatur der Sand nachverdichtet und kleine Hohlräume unterhalb der Decke gebildet haben. Die Test-VOC könnten solche Hohlräume als Diffusionsweg genutzt haben und wären dann nicht von der SPME-Faser erfasst wurden.

Die Ergebnisse zur Diffusion zeigten allerdings, dass innerhalb der Apparatur ein Stofffluss von 3-Octanon zu messen war. Im Vergleich der ermittelten Werte der Diffusionskoeffizienten von ca. $5 * 10^{-8} \mathrm{~cm}^{2} \mathrm{~s}^{-1}$ bei der ersten Messung und ca. $3 * 10^{-6} \mathrm{~cm}^{2} \mathrm{~s}^{-1}$ bei der zweiten Messung mit Trichlorethylen (besitzt eine ähnliche Molmasse wie 3-Octanon von $131,4 \mathrm{~g} \mathrm{~mol}^{-1}$ ) in Luft von $8,75 * 10^{-2} \mathrm{~cm}^{2} \mathrm{~s}^{-1}$ zeigt sich, dass Sand die Diffusionsgeschwindigkeit um den Faktor 10.000 bis 1.000 .000 verlangsamt hat. Typische Diffusionskoeffizienten liegen für Gase zwischen $5 * 10^{-2}$ und $10^{-1} \mathrm{~cm}^{2} \mathrm{~s}^{-1}$, bei Festkörpern zwischen $10^{-10}$ und $10^{-6} \mathrm{~cm}^{2} \mathrm{~s}^{-1}$ und zwischen $10^{-6}$ und $10^{-5} \mathrm{~cm}^{2} \mathrm{~s}^{-1}$ für Flüssigkeiten 266. Auch hier wird deutlich, dass die ermittelten Diffusionskoeffizienten wesentlich niedriger waren als sie für Gase typisch sind. Somit muss der Sand einen maßgeblichen Einfluss auf die Diffusion gehabt haben. Mögliche Silikate an der Oberfläche der Sandkörner könnten 
zur Adsorption von VOC führen [267], wodurch die Diffusion eingeschränkt werden würde. Ebenso können äußere, Ergebnis beeinflussende Faktoren, beispielsweise Konvektion, nicht ausgeschlossen werden.

Die Ergebnisse der Konstanten mittels des 2. Fick'schen Gesetzes zeigten, dass es bei beiden Wiederholungsmessungen jeweils einen Ausreißer bei der Distanz von $10 \mathrm{~cm}$ gab. Die restlichen Konstanten liegen relativ dicht beieinander, weshalb davon ausgegangen werden kann, dass die Diffusionswiderstände über die gesamte Apparatur hinweg ähnlich waren. Gründe für die Ausreißer bei der Distanz von $10 \mathrm{~cm}$ konnten nicht festgestellt werden.

Für das Nicht-Detektieren von 3-Octanon ab der dritten Wiederholungsmessung konnte keine direkte Ursache festgestellt werden. Aufgrund dessen, dass bei den Wiederholungsmessungen mit 3-Octanol dieses VOC in den Proben nicht nachgewiesen werden konnte, wurde spurenanalytisch nach einer Ursache gesucht. Bei der Beprobung der verwendeten Drahtgehäuse, in denen sich die „Quell“" befand, konnte nach den Messungen 3-Octanol detektiert werden. Fehler bei der Pipettierung der Testsubstanz auf das Filterpapier und beim Ansetzten der Verdünnungsreihen konnten somit ausgeschlossen werden. Im Gegensatz zu den Sandproben aus der Startposition konnte $10 \mathrm{~cm}$ links und rechts von der Startposition kein 3-Octanol nachgewiesen werden (Tab. 21 und 22). Des Weiteren konnte Dodecan, ein wenig reaktives und schwer abbaubares Alkan 259, das ein Bestandteil des bei der Verdünnung verwendeten Paraffins ist, in den Proben der Spurenanalytik nicht identifiziert werden. Beides deutet darauf hin, dass die Beweglichkeit innerhalb des Probesubstrates eingeschränkt wurde und sich die Testsubstanz an das Probesubstrat angelagert hat bzw. von diesem absorbiert wurde. Ein systematischer Fehler kann ausgeschlossen werden, da nach jedem Versuch die Apparatur komplett gereinigt und der Versuch nach gleichen Bedingungen wieder aufgebaut wurde. Dabei wurde die Apparatur mit neuem Probesubstrat befüllt und die SPME-Fasern wurden auf Funktionalität überprüft.

Da weniger eine methodische Ursache als Fehlerquelle in Betracht kommt, könnten biologische Abbauprozesse eine Rolle spielen. Trotz gründlicher Reinigung der Apparatur und Ausheizung des Probesubstrates kann nicht ausgeschlossen werden, dass sich möglicherweise ein Biofilm innerhalb der Apparatur gebildet hat. Biofilme stellen Lebensgemeinschaften aus verschiedenen Mikroorganismen dar und können an unterschiedlichen Stoffumwandlungen und -kreisläufen beteiligt sein [268]. Durch einen Biofilm können diffusive Prozesse behindert worden sein und zum Abbau der Testsubstanz geführt haben. Neben biologischen Abbauprozessen können auch andere physikalische Stofftransporte nicht ausgeschlossen werden. Durch die Spurenanalytik konnte keine direkte Ursache für die Hinderung in der Bodenbeweglichkeit von 3-Octanol gefunden werden. In weiterfüh- 
renden Messungen sollten die hier diskutierten möglichen Ursachen weiter untersucht werden.

Durch die unterschiedlichen Adsorptionseigenschaften der SPME-Fasern war eine absolute Quantifizierung der Ergebnisse nicht möglich. Für eine bessere Quantifizierung aufgrund der unterschiedlichen Anreicherungseigenschaften der SPME-Fasern ist eine ausführliche Kalibrierung wichtig, damit dies besser in den Berechnungen berücksichtigt werden kann. Ein Einfluss des Probesubstrates auf die Messergebnisse kann nicht ausgeschlossen werden. Das Probesubstrat hatte eine Körnung von 0-2 mm. Durch Sieben des Substrates auf eine festgelegte Korngröße hätte der Einflussfaktor besser kalkuliert werden können. Des Weiteren sollte das Probesubstrat zusätzlich noch autoklaviert werden, um einen sterileren Zustand zu gewährleisten.

Unter natürlichen Bodenbedingungen beeinflussen der Wassergehalt und das organische Material im Boden zusätzlich die Diffusion von chemischen Substanzen 267] und somit auch die Bodenbewegung der VOC. Damit die Bedeutung der VOC-Bodenbeweglichkeit für die unterirdische Kommunikation zwischen Rhizosphäre und Bodenorganismen besser beurteilt werden kann, sind weitere Messungen nötig. Besonders Messungen unter Verwendung von Mutterboden sind notwendig, um die Informationen besser auf natürliche Prozesse im Freiland transferieren zu können. 


\subsection{Zusammenfassung}

Bisher ist wenig über Boden-VOC, z. B. über ihre Quantität und welche Faktoren ihre Diffusion und Emission beeinflussen können, bekannt, obwohl diese einen wichtigen Einfluss auf die abiotischen und biotischen Prozesse im Boden haben können [188. In diesem Kapitel konnten erstmals für einen Buchenbestand sowohl im Frühjahr als auch im Herbst 42 Boden-VOC identifiziert und für das VOC 3-Octanon die Diffusion in einem definierten Versuchsboden nachgewiesen werden.

Die in der Untersuchung gefundenen VOC konnten anhand von Literatur verschiedenen Akteuren (Bakterien, Pilze und Buchenwurzeln) zugeordnet werden (Anhang A.4). Das Feinwurzel-VOC-Muster von F. sylvatica wurde von fünf Stoffklassen dominiert. Dazu gehörten Terpene und Aldehyde, deren Bedeutung für die Interaktion zwischen den verschiedenen Akteuren im Erdreich bekannt ist. Beide Stoffklassen deuten auf die Anwesenheit von unterschiedlichen Akteuren im Waldboden hin. Somit kann die Anfangshypothese, dass die gemessenen Wurzel-VOC auf die Anwesenheit von Pilzen und Bakterien hindeuten können, bestätigt werden.

In dieser Arbeit wurde nicht nur das VOC-Muster für Feinwurzeln von F. sylvatica untersucht, sondern auch, ob dieses sich mit variierenden Niederschlagsmengen verändert. Im Bezug auf die Fragestellung, welchen Einfluss variierende Niederschlagsmengen auf die Emissionen von Wurzel-VOC haben, zeigten die Ergebnisse, dass die jährliche Niederschlagsmenge einen signifikanten Einfluss auf die identifizierten VOC hat. Mögliche pilzbürtige VOC waren dominant im Untersuchungsgebiet Klötze mit geringer jährlicher Niederschlagsmenge. Dies deutet auf ein größeres Vorkommen von Pilzen in diesem Gebiet hin. In Sellhorn mit hohen jährlichen Niederschlagsmengen waren dagegen die Abundanzen der VOC, die eher den Feinwurzeln von F. sylvatica zuzuordnen sind, dominanter. Dies könnte eine größere Produktion an Feinwurzelmasse in Gebieten mit höheren jährlichen Niederschlagsmengen bedeuten und deckt sich mit dem Ergebnis von Hertel et al. (2013). Hertel et al. (2013) fanden in ihrer Studie auf den gleichen Versuchsflächen heraus, dass die Feinwurzelbiomasse mit zunehmender jährlicher Niederschlagsmenge zunimmt 236]. Die Hypothese, dass sich die Emissionen von Wurzeln-VOC von F. sylvatica zwischen Standorten mit niedrigerem jährlichem Niederschlag und mit höheren jährlichen Niederschlag unterscheidet, konnte ebenfalls bestätigt werden.

Nonanal und Decanal, VOC die mit Trockenstress bei Pflanzen in Verbindung stehen, wurden auf der Fläche mit der längsten Trockenperiode im Untersuchungsjahr 2013 gemessen und können mögliche Marker-VOC für Trockenheit sein. Die Hypothese, dass es mindestens ein Marker-VOC gibt, welches bei der Erkennung von weniger gut wasserversorgten Wurzeln dienen kann, kann angenommen werden.

Nicht nur der Niederschlag spielt eine Rolle bei der Freisetzung von pflanzenbürti- 
gen VOC, sondern auch die Phänologie der Pflanze selbst. Im Hinblick auf die dritte Fragestellung, ob sich die Zusammensetzung des Wurzel-VOC-Musters von F. sylvatica zwischen Herbst und Frühjahr unterscheidet, konnten die Ergebnisse keine Unterschiede im VOC-Muster der Feinwurzeln nachweisen. Es konnten nur signifikante Unterschiede in den Abundanzen der VOC zwischen Herbst und Frühling und den Untersuchungsflächen festgestellt werden. Die Hypothese, dass sich die Zusammensetzung des VOC-Musters saisonal ändert, muss somit verworfen werden.

In dieser Studie wurde das Feinwurzel-VOC-Muster von F. sylvatica untersucht und der Fragestellung nachgegangen, ob es möglich ist, die Bodenbeweglichkeit von Wurzel-VOC zu bestimmen. Es konnte mittels einer neu entwickelten Apparatur die Bodenbeweglichkeit des Wurzel-VOC 3-Octanon im Probesubstrat Sand nachgewiesen werden. Nach 72 Stunden erreichten $0,07 \%$ der Testsubstanz $\left(10^{-3} ; 200 \mu \mathrm{l}\right)$ eine Distanz von $40 \mathrm{~cm}$. Übertragen auf einen sandigen Waldboden würde das bedeuten, dass innerhalb von drei Tagen ein VOC wie 3-Octanon ein Volumen von $1 \mathrm{~m}^{3}$ im sandigen Boden passieren könnte. Für Bodenorganismen, die in der Bodenstreu leben, wird angenommen, dass sie während ihrer Lebenszeit ein ähnlich großes Bodenvolumen durchqueren [269]. Daher kann die Hypothese, dass Wurzel-VOC sich durch den Boden bewegen können und dadurch eine gewisse Reichweite für Interaktionen von Bodenorganismen und Wurzeln besitzen, bestätigt werden.

Zu berücksichtigen ist dabei, dass sich das Zeitregime oberhalb des Erdreiches von dem im Boden unterscheidet. Im Boden braucht ein VOC für die Bewegung deutlich länger als oberirdisch. Oberirdisch verbreitet sich ein VOC bis zu tausend Mal schneller als unterirdisch durch die Bodenporen [270]. Auch Insekten brauchen oberirdisch nur wenige Sekunden, um mehrere Meter weit zu fliegen. Dagegen zeigen Verhaltensversuche mit dem Engerling des Waldmaikäfers (M. hippocastani) im Boden, dass dieser sich innerhalb einer Stunde gerade einmal $10 \mathrm{~cm}$ weit im Erdreich bewegt [42]. Dies stimmt in der Größenordnung mit der Beweglichkeit der VOC im Erdboden überein. 


\section{Olfaktorische Reaktion des}

\section{Buchenstreckfuß Calliteara}

\section{pudibunda (L. 1785) (Lep., \\ Noctuidae) und des Nagelflecks \\ Aglia tau (L. 1761) (Lep., \\ Saturnidae) auf Stamm-VOC der \\ Rot-Buche ( $F$. sylvatica)}

Christine Rachow, Martin Gabriel \& Stefan Schütz
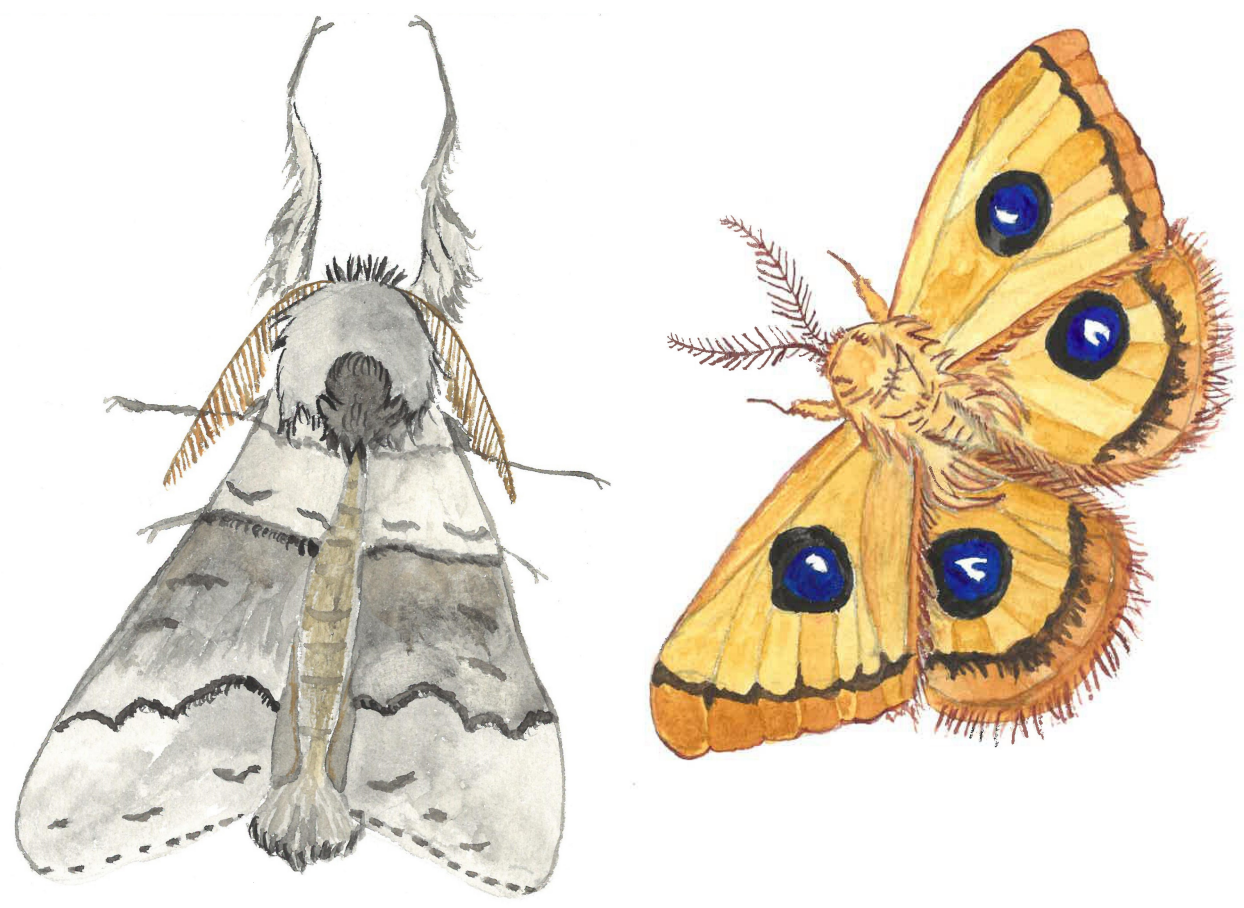

Abbildung 38: Calliteara pudibunda (ox, links) und Aglia tau (o , rechts)

Der Inhalt dieses Kapitels wurde in „Mitteilungen der Deutschen Gesellschaft für allgemeine und angewandte Entomologie" veröffentlicht, Band 19 (2014): 57-62, ISSN: 0344-9084 


\section{Olfactory response of Pale Tussock Calliteara pudibunda (L. 1785) (Lep., Noctuidae) and Tau Emperor Aglia tau (L. 1761) (Lep., Saturnidae) to stem volatiles of beech (Fagus sylvatica) ${ }^{4}$}

Christine Rachow, Martin Gabriel \& Stefan Schütz Georg-August-University, Göttingen

Department of Forest Zoology and Forest Conservation, Büsgen-Institute

Zusammenfassung: Die Antennenreaktionen von zwei verschiedenen Falterarten, dem Streckfuß (Calliteara pudibunda, Lepidoptera, Fam. Noctuidae) und dem Nagelfleck (Aglia tau, Lepidoptera, Fam. Saturniidae) wurden auf die von der Wirtspflanze Buche (Fagus sylvatica, Fagales, Fam. Fagaceae) abgegebenen Stamm-Volatilen mittels eines gekoppelten Gaschromatographen mit einem Quadrupol-Massenspektrometer und einem parallel angeschlossenem elektroantennographischen Detektor GC-MS/EAD untersucht.

Da beide Falter ihre Eier an die Stämme von Buchen legen, fokussiert sich die Untersuchung der Duftwahrnehmung dieser Falter auf Stammvolatile der Buche. Die Antennen des Falters Calliteara pudibunda zeigten auf insgesamt 15 flüchtigen organischen Verbindungen (VOC) der Buchenrinde eine Reaktion. Bei den Antennen des Falters Aglia tau konnten Reaktionen auf insgesamt 39 VOC der Buchenrinde festgestellt werden.

Die Männchen von Calliteara pudibunda sowie beide Geschlechter von Aglia tau reagierten beständig auf (Z)-3-Hexen-1-ol, ein Abbauprodukt von Membranlipiden. Beide Geschlechter von Aglia tau reagierten zusätzlich in über $50 \%$ der untersuchten Antennen auf eine nicht näher bestimmte Substanz und auf 3,3,5-Trimethyl-Cyclohexanon, ein Abbauprodukt von Carotenoiden. Auf drei Substanzen (Limonen, 2-Ethyl-1-hexanol und 3,3,5-Trimethyl-2-Cyclohexen-1-on) reagierten nur die Männchen beider Falterarten.

Die Untersuchung wurde im Rahmen des Projektes „Klimafolgenforschung in Niedersachsen" (KLIFF) durchgeführt.

Key words: Fagus sylvatica, Calliteara pudibunda, Aglia tau, stem volatiles, volatile organic compounds, GC-MS, EAG

Christine Rachow, Georg-August-University, Göttingen, Department of Forest Zoology and Forest Conservation, Büsgen-Institute, Büsgenweg 3, 37077 Göttingen, Germany; E-Mail: crachow@gwdg.de

\footnotetext{
${ }^{4}$ Dieses Kapitel wurde publiziert unter: C. Rachow (2014) / Mitt. Dtsch. Ges. Allg. Angew. Ent. 19, S. $57-62$
} 


\subsection{Introduction}

After spruce (Picea) and pine (Pinus), beech (Fagus sylvatica) is the most frequent deciduous tree species in German forests (Bundeswaldinventur II, BWI II, 271]). The distribution area of beech in central Europe is primarily limited by groundwater availability [6]. The ecogram of forest building trees in central Europe shows that beech is the dominant tree species in the potential natural vegetation at moist to moderately dry conditions [6]. According to recent climate change scenarios beech might be outcompeted in regions where beech is dominant today by more heat and drought tolerant species [11. Beech can be infested by generalist as well as specialist insect species. Specialists are expected to be more strongly affected by physiological changes in their host plants than generalists. Therefore, we focus on the antennal response of the two beech specialist insect species Pale Tussock (Calliteara pudibunda, Fam. Noctuidae) and Tau Emperor (Aglia tau, Fam. Saturniidae), both ovipositing at the stems of beech. Do different specialists use the same stem volatiles for host selection?

In both moths, females seek for the host trees to mate and oviposit at the host trees 272. Both moth species lay their eggs onto the bark of beech trees. After eclosion the caterpillars crawl from the stem to the crown where the larva feed on the leaves. Therefore, volatiles of mixed bark samples collected from breast height and canopy height were trapped on charcoal. In the next step antennae of both species were examined by gaschromatography coupled in parallel to a mass-spectrometer and an electroantennographic detector (GC-MS/EAD) to compare their antennal response to bark volatiles.

\subsection{Material and Methods}

Animals: The used Calliteara pudibunda (Female $\mathrm{N}=2$, Male $\mathrm{N}=5$ ) were caught with a light trap (2 m high gossamer pillar with a $235 \mathrm{~W}$ filament lamp inside) in Billingshäuser Schlucht (Göttingen, Germany) on 03 March 2012. The Aglia tau moths (Female $\mathrm{N}=4$, Male $\mathrm{N}=3$ ) were reared in the institute. The animals were kept at room temperature, daylight conditions and fed with beech leaves. In winter time they were kept in darkness at $6{ }^{\circ} \mathrm{C}$ and 70 to $80 \%$ humidity. Altogether the antennal responses of 12 antennae of Calliteara pudibunda (Female $\mathrm{n}=3$, Male $\mathrm{n}=9$ ) and 13 antennae of Aglia tau (Female $\mathrm{n}=7$, Male $\mathrm{n}=6$ ) were recorded.

Sampling of bark volatiles: Freshly broken bark pieces from ca. 80 years old beech trees were collected at breast height and canopy height in the beginning of the growing season in March 2012. Pieces from breast height and canopy height were mixed in a ratio of 1:1. Three times $63 \mathrm{~g}$ of this bark was filled into $250 \mathrm{ml}$ round bottom flasks. The bark volatiles 
were sampled by a closed-loop-stripping system using $1.8 \mathrm{mg}$ charcoal volatile trap (CLSA Filters, Daumazan, France) and capillary rotary pump (DC 12/16 Fk, Fürgut, Tannheim, Germany) for 3 hours at a flow rate of $1.6 \mathrm{l} \mathrm{min}^{-1}$. Afterwards CLSA samples were eluted with $100 \mathrm{\mu l} \mathrm{CH}_{2} \mathrm{CL}_{2}: \mathrm{CH}_{4} \mathrm{O}(2: 1)$.

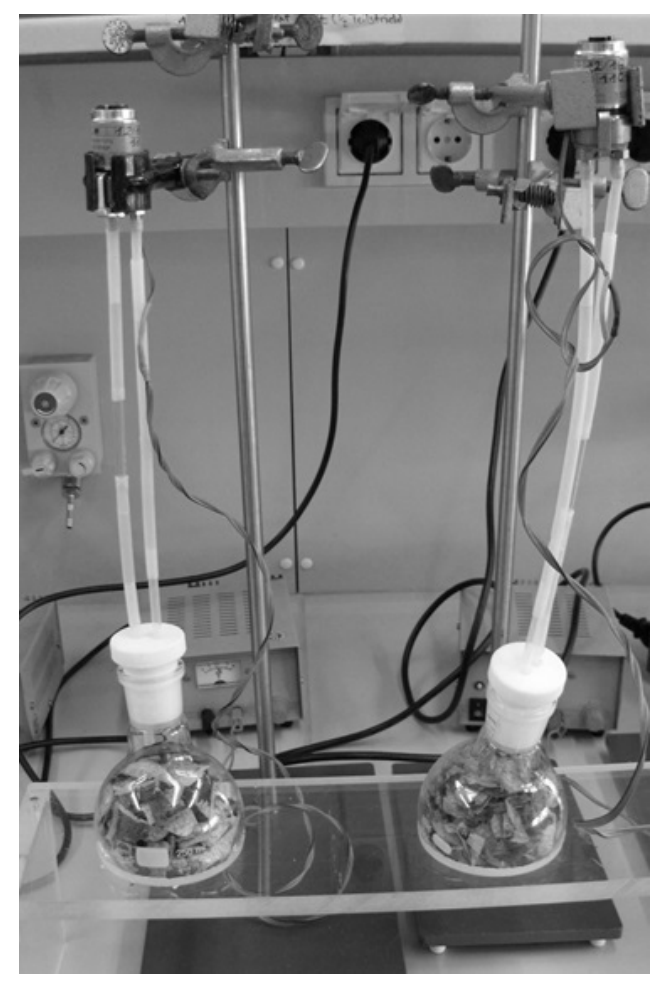

Figure 39: Sampling set up of bark volatiles

GC-MS/EAD: The antennal responses were measured with a coupled GC-MS/EAD combination technique 273. The GC-MS (Agilent, Palo Alto, USA) consists of a type HP6890N gas chromatograph with a polar column (30 m x $0.25 \mathrm{~mm}$ I.D. x $0.25 \mu \mathrm{m}$ film thickness, HP-Innowax, Agilent) connected to a type 5973N quadrupole mass spectrometer with an EI ion-source.

The distal $\mathrm{cm}$ of each antenna was placed into an antenna holder [274]. Within the antenna holder both ends of the antenna were in contact with an insect hemolymph electrolyte solution 275].

Analytical conditions: Volatile samples were injected into the splitless injector in a quantitiy of $1 \mathrm{\mu l}$ at a temperature of $250^{\circ} \mathrm{C}$. The following temperature program started at $50{ }^{\circ} \mathrm{C}$, held for $1.5 \mathrm{~min}$, ramped $7.5^{\circ} \mathrm{C} \mathrm{min}{ }^{-1}$ to $250{ }^{\circ} \mathrm{C}$ and held the end temperature for 5 min. Helium (purity 5.0) was used as a carrier gas. 
Analysis: Identification of bark volatiles was done using the mass spectral library from the National Institute of Standards and Technology (NIST, Gaithersburg, USA) and coelution of authentic standards. Compounds eliciting electroantennographic responses from at least two different antennae were regarded as electrophysiologically active.

\subsection{Results}

Antennae of $C$. pudibunda responded to 15 VOC (Tab. 23). Fourteen volatiles elicited antennal responses exclusively in males and only one volatile in females. However, the data about the female $C$. pudibunda have to be interpreted with care because of only three tested females.

Antennae of $A$. tau responded to 39 volatiles (Tab. 24). Ten volatiles caused antennal responses in the antennae of both sexes. Twentyeight volatiles elicited antennal responses exclusively in males and one volatile exclusively in females. In more than $50 \%$ of the recorded antennae (Z)-3-hexen-1-ol and 3,3,5-trimethyl-cyclohexanone and one not determined volatile elicited responses in both sexes of $A$. tau.

Table 23: Retention index, compound names and number of responding antennae from females and males of Calliteara pudibunda to different beech bark volatiles

\begin{tabular}{|c|c|c|c|c|}
\hline \multicolumn{5}{|c|}{ Calliteara pudibunda } \\
\hline RI & Volatile & Response in & Female $\mathrm{n}=3$ & Male $\mathrm{n}=9$ \\
\hline 1118 & 2-pentanol & $\sigma^{2}$ & 0 & 2 \\
\hline 1209 & limonene* & $\sigma^{\pi}$ & 1 & 3 \\
\hline 1251 & n. d. & $\sigma^{x}$ & 1 & 2 \\
\hline 1386 & (Z)-3-hexen-1-ol* & $\sigma^{2}$ & 1 & 4 \\
\hline 1404 & nonanal* & $\sigma^{7}$ & 0 & 3 \\
\hline 1443 & 2,2-dimethyl-hexanal & $\sigma^{\pi}$ & 0 & 2 \\
\hline 1458 & n. d. & $\sigma^{\pi}$ & 0 & 2 \\
\hline 1475 & 2-ethyl-1-hexanol* & $\sigma^{\pi}$ & 1 & 2 \\
\hline 1481 & pentadecane* $^{*}$ & $\sigma^{x}$ & 1 & 2 \\
\hline 1593 & 3,5,5-trimethyl-2-cyclohexen-1-one & $\sigma^{7}$ & 0 & 2 \\
\hline 1671 & n. d. & 우 & 2 & 0 \\
\hline 1741 & 6,9-heptadecadiene & $\sigma^{x}$ & 1 & 2 \\
\hline 1780 & n. d. & $\sigma^{\pi}$ & 0 & 2 \\
\hline 1864 & n. d. & $\sigma^{\pi}$ & 0 & 2 \\
\hline 1995 & phenol* $^{*}$ & $\sigma^{x}$ & 0 & 3 \\
\hline
\end{tabular}

* volatile identification is confirmed by co-elution with an authentic standard compound, n. d. = not determined

\subsection{Discussion}

In Germany beech is a forest building and economically important tree species. Thus it is desirable to know more about the underlying mechanism of host finding of specialist insect species of beeches. In this study two specialists were compared concerning their antennal responses to beech stem volatiles. No volatile released from beech bark was found to elicite 
Table 24: Retention Index, Compound names and number of responding antennae from females and males of Aglia tau to different beech bark volatiles.

\begin{tabular}{|c|c|c|c|c|}
\hline \multicolumn{5}{|c|}{ Aglia tau } \\
\hline RI & Volatile & Response in & Female $\mathrm{n}=7$ & Male $\mathrm{n}=6$ \\
\hline$<1100$ & n. d. & $\sigma^{x}$ & 1 & 2 \\
\hline 1130 & 1-decene & $90^{x}$ & 3 & 3 \\
\hline 1134 & n. d. & $\sigma^{7}$ & 1 & 3 \\
\hline 1172 & 3-penten-2-ol* & $\sigma^{x}$ & 1 & 4 \\
\hline 1199 & n. d. & $\sigma^{7}$ & 0 & 2 \\
\hline 1209 & limonene* & $\sigma^{x}$ & 1 & 6 \\
\hline 1240 & n. d. & q & 2 & 0 \\
\hline 1250 & n. d. & $90^{7}$ & 4 & 4 \\
\hline 1289 & n. d. & 90 & 2 & 2 \\
\hline 1305 & (E)-4,8-dimethyl-1,3,7-nonatriene & $90^{7}$ & 3 & 6 \\
\hline 1312 & n. d. & $\sigma^{7}$ & 1 & 3 \\
\hline 1336 & N,N-dimethyl-formamide & $\sigma^{7}$ & 0 & 2 \\
\hline 1284 & 2-hydroxy-propanoic acid, ethyl ester & $\sigma^{2}$ & 0 & 4 \\
\hline 1350 & 1-hexanol & qo & 2 & 3 \\
\hline 1355 & methoxy-benzene & $\sigma^{7}$ & 1 & 3 \\
\hline 1364 & n. d. & $\sigma^{7}$ & 0 & 2 \\
\hline 1373 & n. d. & $\sigma^{x}$ & 1 & 3 \\
\hline 1386 & (Z)-3-hexen-1-ol & qo & 4 & 5 \\
\hline 1393 & 3,3,5-trimethyl-cyclohexanone* & Q $0^{x}$ & 6 & 6 \\
\hline 1403 & nonanal* & $90^{x}$ & 3 & 2 \\
\hline 1457 & n. d. & $90^{x}$ & 2 & 2 \\
\hline 1466 & 2-furancarboxaldehyde* & $\sigma^{\top}$ & 0 & 2 \\
\hline 1475 & 2-ethyl-1-hexanol* & $\sigma^{7}$ & 0 & 2 \\
\hline 1493 & 3-ethyl-4-methyl-1-pentanol & $\sigma^{7}$ & 0 & 3 \\
\hline 1529 & 3,3,5-trimethyl-cyclohexanol & $\sigma^{7}$ & 1 & 2 \\
\hline 1537 & 1-octanol* & $\sigma^{7}$ & 0 & 3 \\
\hline 1556 & 2-(2-hydroxypropoxy)-1-propanol & $\sigma^{7}$ & 0 & 2 \\
\hline 1571 & n. d. & $\sigma^{7}$ & 0 & 2 \\
\hline 1575 & n. d. & $\sigma^{7}$ & 0 & 2 \\
\hline 1592 & 3,5,5-trimethyl-2-cyclohexen-1-one & $\sigma^{7}$ & 1 & 2 \\
\hline 1625 & n. d. & $\sigma^{7}$ & 0 & 2 \\
\hline 1675 & heptadecane* & $0^{7}$ & 1 & 2 \\
\hline 1699 & 8-heptadecene & $\sigma^{7}$ & 1 & 3 \\
\hline 1745 & 1,3-dimethoxy-benzene* & $\sigma^{7}$ & 0 & 2 \\
\hline
\end{tabular}

* volatile identification is confirmed by co-elution with an authentic standard compound, n. d. $=$ not determined

antennal responses in both sexes of both moth species. Four volatiles caused in most cases antennal responses in males of $C$. pudibunda and in both sexes of $A$. tau. These volatiles are (Z)-3-hexen-1-ol, nonanal and two not determined volatiles. (Z)-3-hexen-1-ol is wellknown as a degradation product of membrane lipids and therefore as a marker for cell damage. This green leaf odour is frequently released upon mechanical damage and insect herbivore feeding and plays an important role in direct and indirect plant defence [253. Nonanal also being a degradation product of membrane lipids is known to be attractive to other moths like Smerinthus ocellatus $[119$.

Females of $C$. pudibunda showed a sex-specific response only to one volatile, in contrast to females of $A$. tau responding sex-specifically to 11 volatiles. There seems to be no common female specific volatile. Although females of both species live in a similar ecological 
niche the olfactory responses to beech stem volatiles differ markedly. However, with only three females of $C$. pudibunda examined, this result has to be considered with care. Males of C. pudibunda responded to 14 volatiles, most frequently to (Z)-3-hexen-1-ol, nonanal, limonene, and phenol. Limonene is known as a toxic monoterpenoid [276] and phenol as a component of hypersensitive response to pathogen infection [277]. Males of both moth species responded to other volatiles (limonene, 2-ethyl-1-hexanol, 3,3,5-trimethyl-2cyclohexen-1-one) than females with 3,3,5-trimethyl-2-cyclohexen-1-one being known as a degradation product of carotenoids [278.

Remarkably, A. tau differs from $C$. pudibunda in the frequent responses to 3,3,5trimethyl-cyclohexanone which is well-known as degradation product of carotenoids and the frequent responses to (E)-4,8-dimethyl-1,3,7-nonatrien which is known to be a jasmonate pathway controlled compound. It is typically released upon insect attack and very attractive to predators of herbivorous insects $[279]$. Thus, the antennal response spectra of the two species to beech bark volatiles have seven compounds in common mostly belonging to degradation products generated by dying plant cells or compounds related to plant defence. Moreover, the two moth species showed eight (C. pudibunda) and 32 (A. tau) species specific antennal responses to beech bark volatiles. Nowadays $C$. pudibunda tends to infrequent outbreaks 280 and has rarely major impact on forestry. However, climate change scenarios forecast more frequent drought periods. Beech forests might have more drought stress in the future and therefore may be more vulnerable to insect outbreaks. The fact that both moth species respond to volatile compounds that might be related to stress situations of beech trees hints to such an increased vulnerability of stressed beech trees. 


\section{Gesamtdiskussion}

In dieser Studie wurde der Hauptfrage nachgegangen, ob unterschiedliche jährliche Niederschlagsmengen einen Einfluss auf das VOC-Muster von Stämmen und Wurzeln von F. sylvatica besitzt. Dabei wurde nach Stamm- und Wurzel-VOC gesucht, die zur Früherkennung eines durch Trockenheit geschwächten Bestandes dienen können. Des Weiteren wurde untersucht, ob VOC des Stamm-VOC-Musters olfaktorische Reaktionen bei zwei an F. sylvatica vorkommenden Schmetterlingen, Calliteara pudibunda (ein potentielles Schadinsekten an F. sylvatica) und Aglia tau, auslösen. Da beide Schmetterlinge ihre Eier an den Stämmen von F. sylvatica ablegen, sollen die Antennenreaktion bei der Auswahl möglicher Marker-VOC helfen, die auf einen anstehenden Befall mit Schadinsekten hindeuten können. Bisher ist das Stamm-VOC-Muster von Altbuchen im Gegensatz zu den Buchenblättern weitestgehend unerforscht. Daher wurde in dieser Studie eine Stammabsaugkammer in Leichtbauweise für die Beprobung der Stämme von F. sylvatica auf ihr VOC-Muster entwickelt.

In den Jahren, in denen die Stamm- und Wurzel-VOC von F. sylvatica gemessen wurden, gab es keine außergewöhnlich hohe Sommertrockenheit wie in den Jahren 2003 und 2018. Da der Niederschlag in der Vegetationsperiode dafür sorgte, dass F. sylvatica nicht unter Trockenheit zu leiden hatte, bezieht sich die in dieser Arbeit diskutierte Trockenheit auf Standorte mit durchschnittlichen jährlichen Niederschlagsmengen von $<600 \mathrm{~mm}$ im Gegensatz zu Vergleichsstandorten mit $>600 \mathrm{~mm}$. Buchenwälder kommen in Mitteleuropa zwischen 460 bis $>2000 \mathrm{~mm}$ vor [74, allerdings steigt das Trockenheitsrisikio für F. sylvatica bei durchschnittlichen Jahresniederschlagsmengen unter $650 \mathrm{~mm}$ 85.

Für das Stamm-VOC-Muster konnte eine Änderung in der saisonalen VOC-Zusammensetzung festgestellt werden. Einzelne VOC zeigten sowohl in Stamm- als auch in WurzelProben signifikante Abhängigkeiten ihrer Emissionsraten zum Niederschlagsgradienten. Insgesamt haben sich in den VOC-Mustern der Stämme und der Wurzeln sechs mögliche Marker-VOC (vier Terpene: $\alpha$-Pinen und Limonen, Camphen und Campher; zwei Aldehyde: Nonanal und Decanal) herauskristallisiert. Für Limonen und Nonanal konnten olfaktorische Reaktionen von C. pudibunda und A. tau gemessen werden.

Im Folgenden werden alle sechs möglichen Marker-VOC genauer diskutiert.

Die gefundenen Terpene zeigen beim Stamm-VOC-Muster eine deutliche Abhängigkeit zur Jahreszeit. Im Frühling und im Sommer wurden nicht nur mehr Terpene abgegeben, sondern auch die Konzentrationen der Terpene in den Stammproben ( $\alpha$-Pinen, Limonen, Camphen und Campher) waren im Frühling tendenziell höher. Die höheren Konzentrationen können unter anderem auch an den im Durchschnitt 10 Grad wärmeren Tempe- 
raturen im Frühling und Sommer im Gegensatz zum Herbst und Winter liegen. Denn die MT-Emissionen der Pflanzen werden durch den Dampfdruck der MT innerhalb des Pflanzengewebes bestimmt. Der Dampfdruck wiederum wird von der Umgebungstemperatur und der Konzentration der Terpene innerhalb des Pflanzengewebes kontrolliert 281. Dies könnte auch erklären, warum in verschiedenen anderen Studien für die Emissionen von Monoterpenen (MT) von Buchenblättern eine Temperaturabhängigkeit festgestellt wurde 126, 131, 132. Dindorf et al. (2005) wie auch Holzke et al. (2006) konnten in ihren Untersuchungen eine zunehmende Emission von $\alpha$-Pinen und Limonen bei steigenden Temperaturen nachweisen 126, 131. Für Blätter von Quercus ilex maßen Loreto et al. (1996) eine Zunahme der $\alpha$-Pinen-Emissionen um den Faktor 3 bei einer Temperaturerhöhung von 20 auf $30{ }^{\circ} \mathrm{C}$ 282.

Die Konzentrationen der Terpene $\alpha$-Pinen und Limonen waren in den Stamm-Proben im Untersuchungsgebiet mit den geringsten jährlichen Niederschlägen (Calvörde) sowohl auf Sand als auch auf Lehm während des gesamten Untersuchungsjahres höher als auf den anderen Flächen. Im Gegensatz zu den Untersuchungsgebieten Sellhorn und Unterlüß (mit höheren Niederschlagsmengen) weist Calvörde seit 1980 vermehrt negative Zeigerjahre (Jahre mit deutlichen Änderungen in den radialen Zuwachswerten) auf 104. Besonderes in trockeneren Jahren war der radiale Zuwachs gering [104. Die vermehrte Abgabe von $\alpha$-Pinen und Limonen im Untersuchungsgebiet unter Auftreten von vermehrt negativen Zeigerjahren und insgesamt geringen Niederschlagsmengen lässt vermuten, dass beide VOC in Zusammenhang mit dem Fitnesszustand eines Bestandes stehen können.

In anderen Studien werden Terpene mit trocken-gestressten Jungbäumen in Verbindung gebracht. MT-Emissionen von Pflanzen sind bekannt dafür bei Trockenheit zuzunehmen 157 und abzunehmen, wenn der Stress weiterhin anhält oder sich verstärkt, wie beispielsweise bei $\alpha$-Pinen 25, 156,283. Bei allen genannten Studien wurden MT-Emission an Jungbäumen (bis zu 10 Jahre alte Pflanzen) in Töpfen untersucht. Die Trockenheit wurde in den Studien durch das Weglassen des Gießens der Pflanzen von ein bis drei Wochen definiert. Šimpraga et al. (2011) untersuchten die Veränderung von MT-Emissionen von vierjährigen Setzlingen von F. sylvatica bei Nicht-Wässerung von 18 Tagen. Anfangs stiegen die MT-Emissionen an, um dann nach zehn Tagen abzunehmen [25. Ein ähnliches Verhalten zeigten auch zehnjährige Eichen (Quercus ilex) ohne Bewässerung. Auch hier stiegen die MT-Emissionen leicht an, um ab dem achten bis zehnten Tag abzunehmen 283]. Dies deutet darauf hin, dass bei andauernden Trockenstress-Ereignissen die MT-Emissionen von Pflanzen abnehmen. Es kann somit möglich sein, dass im Untersuchungsgebiet Calvörde die erhöhten Konzentrationen von MT auf einen gerade anfangenden Schwächezustand des Bestandes durch Trockenheit hindeuten und sich MT als Marker-VOC zur Früherkennung eignen können. 
Im Untersuchungsgebiet Göhrde, welches in der Mitte des untersuchten Niederschlagsgradienten lag, wurden $\alpha$-Pinen, Camphen und Campher über das gesamte Jahr in höheren Konzentrationen von der Lehmfläche als von der Sandfläche emittiert. $\alpha$-Pinen und Camphen wurden in Studien von Setzlingen ohne dauerhafte Bewässerung vermehrt emittiert 28, 156, 157 und deuten somit als Indikatoren auf Trockenheit hin. Die vermehrte Emission beider VOC kann somit auf eine Zunahme der Trockenheit-Sensibilität auf der feuchteren Lehm-Versuchsfläche im Vergleich zu der Sand-Versuchsfläche in Göhrde hindeuten. Untersuchungen haben belegt, dass besonders auf feuchteren und produktiveren Standorten mit F. sylvatica, wo diese große Wuchshöhen und Durchmesser erreichen, eine zunehmende Wachstumslimitierung stattfindet [284,285]. Eine Ursache könnte die bessere Angepasstheit von F. sylvatica auf Trockenstandorten sein, wodurch sich Sommertrockenheit stärker auf bisher feuchteren Standorten auswirkt, da die Bäume hier physiologisch weniger gut an Trockenheit angepasst sind [285].

Für die VOC-basierte Früherkennung von geschwächten Beständen von F. sylvatica hat sich in dieser Studie $\alpha$-Pinen hervorgehoben. Einerseits wird es vermehrt von den Stämmen im niederschlagsärmeren Untersuchungsgebiet abgegeben, anderseits, weil es auch in der Literatur als trockenstressinduziert bekannt ist. Bereits Mattson \& Haack (1987) vermuteten, dass die Zunahme der $\alpha$-Pinen-Emissionen auf eine zunehmenden Dominanz dieses MT in der Gasphase von gestressten Pflanzen hindeuten kann 14.

Dass $\alpha$-Pinen anlockend auf an F. sylvatica vorkommenden Insekten wirken kann, zeigt zudem eine Untersuchung von Gossner et al. (2014). Gossner et al. fanden heraus, dass bei mit Raupen von Lymantria dispar befallenen Jungbuchen (1,5 m hohe Pflanzen), die $\alpha$-Pinen-Abgabe erhöht war. In ihrer Untersuchung hatten Gossner et al. (2014) die Lockwirkung vom VOC-Muster von jungen befallenen Buchen auf andere Insekten untersucht und konnten nachweisen, dass das VOC-Muster junger befallener Pflanzen von F. sylvatica sich quantitativ und qualitativ ändert und sowohl Parasitoide als auch Antagonisten anlockt [39]. Durch den Raupenfraß wurde die Terpen-Emissionen der Blätter signifikant erhöht. Zudem wurden signifikant mehr Parasitoide in der Nähe von Raupen befallenen F. sylvatica gefangen als bei den Kontrollbäumen. Es kann somit möglich sein, dass Insekten anhand von Terpenen (z. B. $\alpha$-Pinen) nicht nur bereits befallene Buchenbestände, sondern auch gezielt geschwächte Bestände anfliegen können.

Terpene sind ebenfalls bekannt dafür, eine wichtige ökologische Rolle bei der Verteidigung gegen natürliche Feinde zu spielen [286]. Das in den Stamm-VOC-Proben gemessene Limonen hat eine toxische Wirkung auf Pilze, Insekten und Borkenkäfer [15, 287 289. Beim adulten Bergkiefernkäfern (Dendroctonus ponderosae) beträgt die Mortalität, wenn diese 24 Stunden einer Limonen-Konzentration von 12,5 ppm ausgesetzt sind, bereits $34 \%[289$. Von Pflanzen wird dieses MT abgegeben, um sich vor Herbivorenfraß zu schüt- 
zen [287] und um Antagonisten der Herbivoren anzulocken [290, 291]. So kann Limonen als Kairomon für Antagonisten von Herbivoren fungieren. Limonen-Emissionen vom Japanischen Schurbaum (Sophora japonica) infolge von Bockkäferlarvenfraß (Anoplophora glabripennis) dienen als Kairomon für Dastarcus helophoroides 292. Zusätzlich besitzt Limonen eine repellente Wirkung auf das Eiablageverhalten von verschiedenen Insekten [293, 294]. Wirtspflanzen des Rüsselzünslers Diaphania nitidalis die mit Limonen versehen wurden, wurden vom Schmetterling weniger als Eiablageplatz verwendet 293. Bei der Zwiebelfliege (Delia antiqua) verhindert Limonen in Kombination mit zwei weiteren MT (3-Caren und p-Cymen) die Eiablage fast vollständig. Über $95 \%$ der Wirtspflanzen wurden nicht befallen [294].

Möglicherweise kann Limonen zur Verteidigung von F. sylvatica im Untersuchungsgebiet mit den geringsten jährlichen Niederschlagsmengen emittiert worden sein, um sich für einen möglichen anstehenden Herbivorenbefall zu wappnen. Die höhere Abgabe von Limonen in Calvörde könnte somit auf einen Befall mit Schadinsekten hindeuten. Gegensätzlich zu dieser Vermutung ist die Studie von Joó et al. 2010, welcher im Blatt-VOC-Muster von F. sylvatica bei Befall mit Buchenblattlaus (Phyllaphis fagi) weniger Limonen gemessen hat 115. Dies kann im unterschiedlichen Aufbau von Stamm und Blatt und den darin ablaufenden biologischen Prozessen begründet liegen.

Für an F. sylvatica vorkommende herbivore Schmetterlingsarten kann daher angenommen werden, dass es für diese vorteilhaft ist, Limonen wahrzunehmen, um dadurch eine möglichst weniger gut verteidigte Wirtspflanze für eine gute Entwicklung der Larven zu gewährleisten. Die in dieser Studie gemessenen Antennenreaktionen von C. pudibun$d a$ und $A$. tau auf Limonen lassen vermuten, dass ebenfalls Limonen als Marker-VOC zur Früherkennung von Schadorganismen an F. sylvatica genutzt werden kann. Versuche zu der verhaltenssteuernden Wirkung von Limonen auf an F. sylvatica vorkommenden Schmetterlingen sollten zur Überprüfung der Eignung dieses VOC als Marker-VOC in nachfolgenden Untersuchungen durchgeführt werden.

Bei den gefundenen Aldehyden Nonanal und Decanal wurden im Untersuchungsgebiet Klötze (Sand) bei den Wurzel-Messungen signifikant höhere Konzentrationen im Frühling als im Herbst abgegeben. Die Aldehyde zeigten in den Stammmessungen keine Unterschiede in ihren Konzentrationen zu den unterschiedlichen Niederschlagsmengen, sondern nur einen jahreszeitlichen Trend, was vermuten lässt, dass sie Anzeiger für kurzfristige Trockenheitsereignisse sind. Dies würde auch die höhere Konzentration auf Klötze (Sand) im Frühjahr in den Wurzelmessungen bestätigen.

Nonanal konnte in dieser Studie wiederholt Antennenreaktionen vom männlichen Schmetterling $C$. pudibunda als auch von beiden Geschlechtern von $A$. tau hervorrufen. Aldehyde 
wie Nonanal und Decanal sind unter anderem Alterungsmarker, die gebildet werden, wenn es zu einem Zellsterben im Pflanzengewebe kommt [142]. Nonanal wird z. B. vermehrt von verbräunten Blättern von der Rosskastanie (Aesculus hippocastanum) abgegeben [43]. Aldehyde können den Insekten dabei helfen, alternde von jungen oder gesunde von kranken Pflanzen zu unterscheiden. Dadurch können z. B. Schmetterlinge sicherstellen, dass sich ihre Larven von frischen, jungen und unbefallenen Blättern ernähren. Auch das VOCMuster trocken-gestresster Pflanzen ist verhaltenswirksamer für Insekten [14. Es ist daher anzunehmen, dass Nonanal für beide der untersuchten Schmetterlingsarten als Infochemikalie für geschwächte Stämme von F. sylvatica dienen kann.

Bisher wurden nur äußere Einflussfaktoren auf die VOC-Abgabe von F. sylvatica diskutiert. Doch auch die genetische Variation von Rot-Buchenbeständen können einen Einfluss auf das VOC-Muster besitzen besonders da weit verbreitete Baumarten, wie F. sylvatica, eine große genetische Vielfalt und damit ein umfangreiches Anpassungspotential 295] sowohl in bewirtschafteten und als auch in unbewirtschafteten Beständen besitzen auch wenn diese geographisch nahe beieinander liegen [296, 297]. Bei Hybriden von Pappeln konnte in einer Studie von Eller el al. (2012) nachgewiesen werden, dass verschiedene Genotypen unterschiedliche Emissionen von Isoprenen und MT aufwiesen [298].

Für die Untersuchungsgebiete in dieser Studie wurde in einer Untersuchung von Carsjens et al. (2014) eine geringe genetische Variation zwischen den Untersuchungsgebieten, wie dies für bestandsbildende Baumarten mit weiter Pollenverbreitung charakteristisch ist [299], und eine hohe genetische Variation innerhalb der Populationen von F. sylvatica Setzlingen festgestellt [300]. Interessant in der Studie von Carsjens et al. (2014) ist, dass die Expression von Genen, welche die Enzyme kodieren, die für die Reinigung von SuperoxidRadikalen, Wasserstoffperoxide und toxischen Aldehyden erforderlich sind, hoch bei Setzlingen von den Untersuchunsgebieten Sellhorn und Unterlüß und gering bei Setzlingen von trockenen Gebieten (Klötze und Calvörde) unter Trockenstress waren [300].

Es ist durchaus möglich, dass mit Stress in Verbindung stehende Gene einen Einfluss auf das VOC-Muster besitzen. Inwieweit diese das VOC-Muster verändern können ist ein interessanter Forschungsbereich für weitere Studien.

In dieser Arbeit wird die Niederschlagsmenge als Einflussfaktor auf das Stamm- und Wurzel-VOC-Muster von F. sylvatica diskutiert. In dem Untersuchungsjahr 2011 konnte eine zunehmende jährliche Niederschlagssumme auf den Untersuchungsgebieten vom Südosten zum Nordwesten vom Deutschen Wetterdienst gemessen werden. Die jährliche Niederschlagssumme nahm von Calvörde nach Unterlüß zu (Januar bis November: von 416 und $515 \mathrm{~mm}$ auf $543 \mathrm{~mm}$ ). Im Untersuchungsjahr 2013 und 2015, in denen im Herbst bzw. im Frühjahr die Wurzel-VOC gemessen wurden, nahm die jährliche Niederschlags- 
summe ebenfalls von Klötze nach Sellhorn zu (Januar bis Dezember: 2013 von 614 und $676 \mathrm{~mm}$ auf $726 \mathrm{~mm} ; 2015$ von 562 und $670 \mathrm{~mm}$ auf $816 \mathrm{~mm}$ ) 301.

Für zukünftige Untersuchungen zum VOC-Muster entlang eines Niederschlaggradienten wäre es interessant dies anhand mehrerer Untersuchungsgebiete durchzuführen, um sicherzustellen, dass die gemessenen Effekte wirklich von der Niederschlagsmenge abhängen und nicht von anderen Einflussfaktoren.

Ein möglicher Einflussfaktor kann das Insektenaufkommen auf den Untersuchungsgebieten gewesen sein, welches in dieser Arbeit nicht erhoben wurde. Auch wenn keine sichtbaren Schädigungen durch einen Insektenbefall erkennbar waren, kann die Fraßaktivität phytophager Insektenstadien einen Einfluss auf das VOC-Muster gehabt haben, da diese je nach Herbivorenart und chemischen Zusammensetzung ihres Speichels unterschiedliche Duftmuster bei den befallenen Pflanzen auslösen können 302. Auch nicht sichtbare Einflussfaktoren wie Rotkern-Bildung im Buchenstamm kann das Stamm-VOC-Muster beeinflusst haben. Bekannte farbkerntypische VOC für F. sylvatica sind z. B. Nonanal und 1-Octanol 303. Diese möglichen Einflussfaktoren sollten in weiteren Untersuchungen mit aufgenommen werden, um die hier gefundenen möglichen Marker-VOC für unter Trockenstress stehende Buchenbestände zu bestätigen. Des Weiteren sollte in zukünftigen Untersuchungen die sich saisonal verändernde Vegetation, insbesondere Zeigerpflanzen im Bestand, mit dokumentiert werden. Zeigerpflanzen dienen unter anderem als Feuchteanzeiger (z. B. Bärlauch (Allium ursinum)) und können zusätzlich zu den Niederschlagswerten einen Aufschluss zum Wasserhaushalt des jeweiligen Standorts geben.

Ein zusätzlicher Punkt, der bei weiterführenden Arbeiten berücksichtigt werden sollte, ist der Beprobungsplan. Durch das zweistündige Messen des VOC-Musters von Stamm und Wurzel zur Mittagszeit konnten keine Aussagen zum Tagesverlauf des VOC-Musters auf den verschiedenen Untersuchungsgebieten getroffen werden. Es ist anzunehmen, dass das VOC-Muster wie die Photosyntheserate einen tagesabhängigen Verlauf besitzt.

Die Hauptfragestellung, ob es mindestens ein VOC gibt, welches auf einen bevorstehenden Befall mit Schadinsekten an F. sylvatica hindeuten kann, konnte beantwortet werden. Die beiden MT $\alpha$-Pinen und Limonen wurden in höheren Konzentrationen im Stamm-VOC-Muster auf dem niederschlagsärmeren Standort gemessen. $\alpha$-Pinen scheint von Bedeutung zu sein, da es durch Literaturabgleich mit Trockenheitsstress von Bäumen in Verbindung gebracht werden konnte. Das auf den Flächen gemessene Limonen kann aufgrund des Wissens, dass es bei der pflanzlichen Verteidigung gegen Herbivoren von Bedeutung ist und dass es von an F. sylvatica vorkommenden Schmetterlingen wahrgenommen werden kann, als ein interessantes VOC bei der Früherkennung betrachtet werden. Bei den Aldehyden kann Nonanal diese Funktion übernehmen. 


\section{Ausblick}

Die Ergebnisse dieser Studie geben einen ersten Überblick über das Stamm- und WurzelVOC-Muster von Altbäumen von F. sylvatica und zeigen mögliche Interaktionen einzelner VOC zwischen Altbäume und Insekten auf. Die meisten bisherigen Versuche zum VOCMuster von Stämmen und Wurzeln verschiedener Baumarten wurden an jungen Pflanzen und meist in Gewächshäusern durchgeführt. VOC-Emissionen von Stämmen und besonders von Wurzeln von Waldbäumen und ihre ökologische Bedeutung sind weitestgehend unerforscht.

Für weitere Untersuchungen ist es interessant, das VOC-Muster von F. sylvatica in Untersuchungsgebieten mit unterschiedlichen jährlichen Niederschlagsmengen in einem Trockenjahr zu messen und zu überprüfen, ob sich die hier diskutierten VOC-Marker bestätigen. Dabei wäre es von Interesse herauszufinden, ob auf Standorten mit geringeren jährlichen Niederschlagsmengen zu Beginn einer solchen Trockenperiode die hier diskutierten VOC $\alpha$-Pinen, Limonen und Nonanal in erhöhter Menge von den Stämmen emittiert werden und ob in einem solchen Bestand mit höherer Wahrscheinlichkeit mit Schaderregern zu rechnen ist. Der Vorteil des Messens von Marker-VOC ist, mit wenig Aufwand den Zustand eines Bestandes nur durch die Untersuchung der Bestandesluft auf Marker-VOC zu überprüfen.

Eine Möglichkeit, den in dieser Arbeit gewonnen Erkenntnisstand in der praktischen Anwendung zu nutzen, wäre die Messung der Bestandesluft mittels Gassensoren an Spezialdrohnen. Im Projekt ProtectForest wird die drohnengestützte Detektion verwendet, um nach bestimmten VOC (Monoterpenen), die auf einen Borkenkäferbefall an Nadelbäumen hindeuten, zu suchen [304]. Befallene Bäume können dadurch früher aus dem Bestand entnommen werden, bevor sichtbare Schäden am Baum auftreten und die Käferpopulation sich weiter ausbreiteten kann [304]. Vorteil dieser Technik ist, dass sie sensitiv und selektiv ist und gezielt nach einzelnen Stoffgruppen sowie Marker-VOC suchen kann. Für F. sylvatica könnte die Verwendung der Drohnentechnik genutzt werden, um Bestände, die unter Trockenheit leiden, rechtzeitig mittels der oben genannten möglichen MarkerVOC zu erkennen.

Auch in der Landwirtschaft wird bei der Überwachung des Agrarlandes auf Drohnen und zusätzlich auf mobil fahrende Roboter gesetzt, die wie beim Projekt ProtectForest mit einer portablen elektrischen Nase ausgestattet sind und darüber Boden-VOC messen können [305]. Solche intelligenten bodendetektierenden Untersuchungsroboter könnten möglicherweise auch im Wald genutzt werden, um Marker-VOC von durch Trockenheit geschädigte Wurzeln wahrzunehmen und es dadurch ermöglichen, Schädigungen von Waldbäumen frühzeitig über den Zustand der Wurzel zu erkennen. 


\section{Literaturverzeichnis}

[1] Anonymus: Was sind Trüffelbäume? Website, 2018. - Online unter www.trüffelbaumschule.net; abgerufen am 10. August 2018

[2] Müller-Haubold, H ; Hertel, D ; Seidel, D ; Knutzen, F ; Leuschner, C: Climate responses of aboveground productivity and allocation in Fagus sylvatica: a transect study in mature forests. In: Ecosystems 16 (2013), Nr. 8, S. 1498-1516

[3] Stocker, Thomas F. ; Qin, Dahe ; Plattner, Gian-Kasper ; Tignor, Melinda M. B. ; Allen, Simon K. ; Boschung, Judith ; Nauels, Alexander ; XiA, Yu ; Bex, Vincent ; Midgley, Pauline M.: Climate change 2013: The physical science basis. Working group I Contribution to the fifth assessment report of the intergovernmental panel on climate change. New York, USA: Cambridge University Press, 2014. - 1535 S. - ISBN 978-1-107-05799-9

[4] Wagner, SVEN ; Fischer, HOLGER: Klimawandel - wie reagiert der Waldbau. In: ProWALD 3 (2007), Nr. 2007, S. 4-7

[5] BrÄsICKE, Nadine ; Wulf, Alfred: 4.8 Über die Zunahme thermophiler Schadorganismen in Wäldern - Umbaupläne müssen dies berücksichtigen. In: Warnsignal Klima: Gefahren für Pflanzen, Tiere und Menschen. 2. Aufl. Lozán, J. L.; Grassl, H.; Karbe, L.; Jendritzky, G., 2014, S. 1-9. - Elektron. Veröffent. http://www. warnsignal-klima.uni-hamburg.de

[6] Ellenberg, Heinz ; Leuschner, Christoph: Vegetation Mitteleuropas mit den Alpen: In ökologischer, dynamischer und historischer Sicht. 6. Aufl. Stuttgart, DE: Eugen Ulmer Verlag, 2010. - 1334 S. - ISBN 978-3-8252-8104-5

[7] Fotelli, Mariangela N. ; Gessler, Arthur ; Peuke, Andreas D. ; Rennenberg, Heinz: Drought affects the competitive interactions between Fagus sylvatica seedlings and an early successional species, Rubus fruticosus: responses of growth, water status and $\delta 13 \mathrm{C}$ composition. In: New Phytol. 151 (2001), Nr. 2, S. 427-435

[8] Sutmöller, Johannes ; Spellmann, Hermann ; Fiebiger, Caroline ; Albert, Matthias: Der Klimawandel und seine Auswirkungen auf die Buchenwälder in Deutschland. The effects of climate change on beech forests in Germany. In: NWFVA (Hrsg.): Ergebnisse angewandter Forschung zur Buche. Bd. 3. Göttingen, DE: Universitätsverlag Göttingen, 2008. - ISBN 978-3-940344-44-1, S. 135-157 
[9] Bréda, Nathalie ; Huc, Roland ; Granier, André ; Dreyer, Erwin: Temperate forest trees and stands under severe drought: a review of ecophysiological responses, adaptation processes and long-term consequences. In: Ann For. Sci. 63 (2006), Nr. 6, S. 625-644

[10] Fotelli, M N. ; Rennenberg, H ; GeßLer, A: Effects of drought on the competitive interference of an early successional species (Rubus fruticosus) on Fagus sylvatica L. seedlings: ${ }^{15} \mathrm{~N}$ uptake and partitioning, responses of amino acids and other N compounds. In: Plant Bio. 4 (2002), Nr. 3, S. 311-320

[11] Geßler, Arthur ; Keitel, Claudia ; Kreuzwieser, Jürgen ; Matyssek, Rainer ; Seiler, Wolfgang ; RennenberG, Heinz: Potential risks for European beech (Fagus sylvatica L.) in a changing climate. In: Trees 21 (2007), Nr. 1, S. 1-11

[12] Krengel, Sandra ; Seidel, Petra: 3.14 Über die Zunahme thermophiler Schadorganismen in Wäldern am Beispiel der Borkenkäfer. In: Warnsignal Klima: Biodiversität. Lozán, J. L.; Breckle, S. W.; Müller, R.; Rachor, E., 2016, S. 184-189. Elektron. Veröffent. - http://www. warnsignal-klima.uni-hamburg.de

[13] John, R ; Delb, H ; Hielscher, K ; Hurling, R ; Lobinger, G ; Niesar, M ; Otto, LF ; Petercord, R ; Thiel, J: Borkenkäfer an Nadelbäumen erkennen, vorbeugen, kontrollieren. 8. Aufl. Bonn, DE: aid Infodienst, 2016. - 54 S. - ISBN 978-8308-1238-8

[14] Mattson, William J. ; HAACK, Robert A.: The role of drought in outbreaks of plant-eating insects. In: BioScience 37 (1987), Nr. 2, S. 110-118

[15] Mattson, William J. ; HAack, Robert A.: The role of drought stress in provoking outbreaks of phytophagous insects. In: Barbosa, P (Hrsg.) ; Schultz, J. C. (Hrsg.): Insect outbreaks. San Diego, USA: Academic Press San Diego, 1987. ISBN 0-12-078148-4, S. 365-407

[16] REdDy, Gadi V. ; Guerrero, Angel: Interactions of insect pheromones and plant semiochemicals. In: Trends Plant Sci. 9 (2004), Nr. 5, S. 253-261

[17] Klaschka, Ursula: Spurenstoffe beeinflussen des Zusammenleben. In: Nachr. Chem. 59 (2011), Nr. 6, S. 613-618

[18] RenwiCK, JAA: Chemical ecology of oviposition in phytophagous insects. In: Experientia 45 (1989), Nr. 3, S. 223-228 
[19] Rennenberg, H ; loreto, F ; Polle, A ; Brilli, F ; Fares, S ; Beniwal, RS ; Gessler, A: Physiological responses of forest trees to heat and drought. In: Plant Biol. 8 (2006), Nr. 5, S. 556-571

[20] Weldegergis, Berhane T. ; Zhu, Feng ; Poelman, Erik H. ; Dicke, Marcel: Drought stress affects plant metabolites and herbivore preference but not host location by its parasitoids. In: Oecologia 177 (2015), Nr. 3, S. 701-713

[21] Bonello, Pierluigi ; Mcnee, William R. ; Storer, Andrew J. ; Wood, David L. ; Gordon, Thomas R.: The role of olfactory stimuli in the location of weakened hosts by twig-infesting Pityophthorus spp. In: Ecol. Entomol. 26 (2001), Nr. 1, S. $8-15$

[22] Ninnemets, Ülo: Mild versus severe stress and BVOCs: thresholds, priming and consequences. In: Trends Plant Sci. 15 (2010), Nr. 3, S. 145-153

[23] Ninnemets, Ülo ; Kännaste, Astrid ; Copolovici, Lucian: Quantitative patterns between plant volatile emissions induced by biotic stresses and the degree of damage. In: Front. Plant Sci. 4 (2013), Nr. 262, S. 1-15

[24] Karl, Thomas ; Guenther, A ; Turnipseed, A ; Patton, EG ; Jardine, Kolby: Chemical sensing of plant stress at the ecosystem scale. In: Biogeosci. Discuss. 5 (2008), Nr. 3, S. 2381-2399

[25] Šimpraga, M ; Verbeeck, Hans ; Demarcke, Marie ; Joó, Éva ; Pokorska, Olga ; Amelynck, Crist ; Schoon, Niels ; Dewulf, Jo ; Van Langenhove, Herman ; Heinesch, Bernard ; Aubinet, M ; Müller, J-F ; Steppe, K: Clear link between drought stress, photosynthesis and biogenic volatile organic compounds in Fagus sylvatica L. In: Atmos. Environ. 45 (2011), Nr. 30, S. 5254-5259

[26] Copolovici, Lucian ; KÄnnaste, Astrid ; Remmel, Triinu ; Ninnemets, Ülo: Volatile organic compound emissions from Alnus glutinosa under interacting drought and herbivory stresses. In: Environ. Exp. Bot. 100 (2014), S. 55-63

[27] Bourtsoukidis, E ; Kawaletz, H ; Radacki, D ; Schütz, S ; Hakola, H ; Hellén, H ; Noe, S ; Mölder, I ; Ammer, C ; Bonn, Boris: Impact of flooding and drought conditions on the emission of volatile organic compounds of Quercus robur and Prunus serotina. In: Trees 28 (2014), Nr. 1, S. 193-204

[28] Ormeño, E ; Mevy, JP ; Vila, B ; Bousquet-Mélou, A ; Greff, S ; Bonin, $\mathrm{G}$; FERnAndez, C: Water deficit stress induces different monoterpene and sesqui- 
terpene emission changes in Mediterranean species. Relationship between terpene emissions and plant water potential. In: Chemosphere 67 (2007), Nr. 2, S. 276-284

[29] Staudt, Michael ; Ennajah, Amel ; Moulllot, Florent ; Joffre, Richard: Do volatile organic compound emissions of Tunisian cork oak populations originating from contrasting climatic conditions differ in their responses to summer drought? In: Can. J. For. Res. 38 (2008), Nr. 12, S. 2965-2975

[30] Niedersächsisches Ministerium für Wissenschaft und Kultur: KLIFF Klimafolgenforschung in Niedersachsen. - Online erhältlich unter http://www. kliff-niedersachsen.de.vweb5-test.gwdg.de; abgerufen am 6. Juli 2018.

[31] Heinrich-Ramm, R. ; Jakubowski, M. ; Heinzow, B. ; Molin Christensen, J. ; Olsen, E. ; Hertel, O.: Biological monitoring for exposure to volatile organic compounds (VOCs). In: Pure Appl. Chem. 72 (2000), Nr. 3, S. 385-436

[32] Knudsen, Jette T. ; Eriksson, Roger ; Gershenzon, Jonathan ; StÅhl, Bertil: Diversity and distribution of floral scent. In: Bot. Rev. 72 (2006), Nr. 1, S. 1-120

[33] Pichersky, Eran ; Noel, Joseph P. ; Dudareva, Natalia: Biosynthesis of plant volatiles: nature's diversity and ingenuity. In: Science 311 (2006), Nr. 5762, S. 808-811

[34] Dudareva, Natalia ; Klempien, Antje ; Muhlemann, Joëlle K ; Kaplan, Ian: Biosynthesis, function and metabolic engineering of plant volatile organic compounds. In: New Phytol. 198 (2013), Nr. 1, S. 16-32

[35] Dicke, Marcel ; BALdwin, Ian T.: The evolutionary context for herbivore-induced plant volatiles: beyond the 'cry for help'. In: Trends Plant Sci. 15 (2010), Nr. 3, S. $167-175$

[36] FARmer, Edward E.: Surface-to-air signals. In: Nature 411 (2001), Nr. 6839, S. 854

[37] Francke, Wittko ; Dettner, Konrad: Chemical signalling in beetles. In: The Chemistry of Pheromones and Other Semiochemicals II. Topics in Current Chemistry. Berlin, Heidelberg, DE: Springer, 2005. - ISBN 978-3-540-21308-6, S. $85-166$

[38] Heuskin, Stéphanie ; Verheggen, François J ; Haubruge, Eric ; Wathelet, Jean-Paul ; LognAY, Georges: The use of semiochemical slow-release devices in integrated pest management strategies. In: Biotechnol. Agron. Soc. Environ. 15 (2011), Nr. 3, S. 459-470 
[39] Gossner, Martin M. ; Weisser, Wolfgang W. ; Gershenzon, Jonathan ; UnSICKER, Sybille B.: Insect attraction to herbivore-induced beech volatiles under different forest management regimes. In: Oecologia 176 (2014), Nr. 2, S. 569-580

[40] Dindorf, T ; Kuhn, U ; Ganzeveld, L ; Schebeske, G ; Ciccioli, P ; Holzke, C ; Köble, R ; Seufert, G ; Kesselmeier, John: Significant light and temperature dependent monoterpene emissions from European beech (Fagus sylvatica L.) and their potential impact on the European volatile organic compound budget. In: J. Geophys. Res. Atmos. 111 (2006), Nr. D16, S. 1-15

[41] Llusià, J ; Peñuelas, J ; Alessio, GA ; Ogaya, R: Species-specific, seasonal, inter-annual, and historically-accumulated changes in foliar terpene emission rates in Phillyrea latifolia and Quercus ilex submitted to rain exclusion in the Prades Mountains (Catalonia). In: Russ. J. Plant Physiol. 58 (2011), Nr. 1, S. 126-132

[42] Weissteiner, Sonja ; Huetteroth, Wolf ; Kollmann, Martin ; Weissbecker, Bernhard ; Romani, Roberto ; Schachtner, Joachim ; Schütz, Stefan: Cockchafer larvae smell host root scents in soil. In: PLoS One 7 (2012), Nr. 10, S. e45827

[43] Johne, A B. ; Weissbecker, Bernhard ; Schütz, Stefan: Volatile emissions from Aesculus hippocastanum induced by mining of larval stages of Cameraria ohridella influence oviposition by conspecific females. In: J. Chem. Ecol. 32 (2006), Nr. 10, S. 2303-2319

[44] Zhao, Nan ; Guan, Ju ; Ferrer, Jean-Luc ; Engle, Nancy ; Chern, Mawsheng ; Ronald, Pamela ; Tschaplinski, Timothy J. ; Chen, Feng: Biosynthesis and emission of insect-induced methyl salicylate and methyl benzoate from rice. In: Plant Physiol. Biochem. 48 (2010), Nr. 4, S. 279-287

[45] Kost, C ; Heil, M: Herbivore-induced plant volatiles induce an indirect defence in neighbouring plants. In: J. Ecol. 94 (2006), Nr. 3, S. 619-628

[46] Frost, Christopher J. ; Appel, Heidi M. ; Carlson, John E. ; De Moraes, Consuelo M. ; Mescher, Mark C. ; Schultz, Jack C.: Within-plant signalling via volatiles overcomes vascular constraints on systemic signalling and primes responses against herbivores. In: Ecol. Lett. 10 (2007), Nr. 6, S. 490-498

[47] Heil, Martin ; Bueno, Juan Carlos S.: Within-plant signaling by volatiles leads to induction and priming of an indirect plant defense in nature. In: Proc. Natl. Acad. Sci. USA 104 (2007), Nr. 13, S. 5467-5472 
[48] Unsicker, Sybille B. ; Kunert, Grit ; Gershenzon, Jonathan: Protective perfumes: the role of vegetative volatiles in plant defense against herbivores. In: Curr. Opin. Plant Biol. 12 (2009), Nr. 4, S. 479-485

[49] Degenhardt, Jörg ; Gershenzon, Jonathan ; Baldwin, Ian T. ; Kessler, André: Attracting friends to feast on foes: engineering terpene emission to make crop plants more attractive to herbivore enemies. In: Curr. Opin. Biotechnol. 14 (2003), Nr. 2, S. 169-176

[50] Pichersky, Eran ; Gershenzon, Jonathan: The formation and function of plant volatiles: perfumes for pollinator attraction and defense. In: Curr. Opin. Plant Biol. 5 (2002), Nr. 3, S. 237-243

[51] WeI, Jianing ; Wang, Lizhong ; Zhu, Junwei ; Zhang, Sufang ; NAndi, Owi I. ; KANG, Le: Plants attract parasitic wasps to defend themselves against insect pests by releasing hexenol. In: PLOS one 2 (2007), Nr. 9, S. e852

[52] Agrawal, Anurag A.: Mechanisms, ecological consequences and agricultural implications of tri-trophic interactions. In: Curr. Opin. Plant Biol. 3 (2000), Nr. 4, S. 329-335

[53] Takabayashi, Junji ; Dicke, Marcel: Plant-carnivore mutualism through herbivore-induced carnivore attractants. In: Trends Plant Sci. 1 (1996), Nr. 4, S. 109-113

[54] Tumlinson, James H. ; Lewis, W J. ; Vet, Louise E.: How parasitic wasps find their hosts. In: Sci. Am. 268 (1993), Nr. 3, S. 100-106

[55] Zhang, Qing-He ; Schlyter, Fredrik: Inhibition of predator attraction to kairomones by non-host plant volatiles for herbivores: a bypass-trophic signal. In: PloS one 5 (2010), Nr. 6, S. e11063

[56] Schroeder, L M.: Differences in responses to $\alpha$-pinene and ethanol, and flight periods between the bark beetle predators Thanasimus femoralis and T. formicarius (Col.: Cleridae). In: For. Ecol. Manage. 177 (2003), Nr. 1-3, S. 301-311

[57] Germinara, Giacinto S. ; De Cristofaro, Antonio ; Rotundo, G: Antennal olfactory responses to individual cereal volatiles in Theocolax elegans (Westwood)(Hymenoptera: Pteromalidae). In: J. Stored Prod. Res. 45 (2009), Nr. 3, S. 195-200 
[58] Lu, Peng-Fei ; Huang, Ling-Qiao ; Wang, Chen-Zhu: Identification and field evaluation of pear fruit volatiles attractive to the oriental fruit moth, Cydia molesta. In: J. Chem. Ecol. 38 (2012), Nr. 8, S. 1003-1016

[59] Ruther, Joachim ; Reinecke, Andreas ; Hilker, Monika: Plant volatiles in the sexual communication of Melolontha hippocastani: response towards time-dependent bouquets and novel function of (Z)-3-hexen-1-ol as a sexual kairomone. In: Ecol. Entomol. 27 (2002), Nr. 1, S. 76-83

[60] Huang, Cui-Hong ; Yan, Feng-Ming ; Byers, John A. ; Wang, Rong-Jiang ; Xu, Chong-Ren: Volatiles induced by the larvae of the Asian corn borer (Ostrinia furnacalis) in maize plants affect behavior of conspecific larvae and female adults. In: Insect Science 16 (2009), Nr. 4, S. 311-320

[61] Webster, Ben ; Bruce, Toby J A. ; Pickett, John A. ; Hardie, Jim: Volatiles functioning as host cues in a blend become nonhost cues when presented alone to the black bean aphid. In: Anim. Behav. 79 (2010), Nr. 2, S. 451-457

[62] Bruce, Toby J A. ; Pickett, John A.: Perception of plant volatile blends by herbivorous insects-finding the right mix. In: Phytochemistry 72 (2011), Nr. 13, S. $1605-1611$

[63] Fraser, Ann M. ; Mechaber, Wendy L. ; Hildebrand, John G.: Electroantennographic and behavioral responses of the sphinx moth Manduca sexta to host plant headspace volatiles. In: J. Chem. Ecol. 29 (2003), Nr. 8, S. 1813-1833

[64] Birkett, Michael A. ; Bruce, Toby J A. ; Martin, Janet L. ; Smart, Lesley E. ; OAKLEY, JON ; WADHAMS, Lester J.: Responses of female orange wheat blossom midge, Sitodiplosis mosellana, to wheat panicle volatiles. In: J. Chem. Ecol. 30 (2004), Nr. 7, S. 1319-1328

[65] Quiroz, A ; Pettersson, J ; Pickett, J A. ; Wadhams, L J. ; Niemeyer, H M.: Semiochemicals mediating spacing behavior of bird cherry-oat aphid, Rhopalosiphum padi feeding on cereals. In: J. Chem. Ecol. 23 (1997), Nr. 11, S. 2599-2607

[66] Ebel, Robert C. ; Mattheis, James P. ; Buchanan, David A.: Drought stress of apple trees alters leaf emissions of volatile compounds. In: Physiologia Plantarum 93 (1995), Nr. 4, S. 709-712

[67] Schütz, S ; Weissbecker, B ; Apel, K H. ; Wenk, M: Duftstoffsignale als Marker für die Befallsdisposition von Kiefern durch den Blauen Kiefernprachtkäfer 
Phaenops cyanea F. (Col., Buprestidae). In: Mitt. Dtsch. Ges. Allg. Angew. Ent. 14 (2004), S. 301-306

[68] Copolovici, Lucian ; Kännaste, Astrid ; Remmel, Triinu ; Vislap, Vivian ; Ninnemets, Ülo: Volatile emissions from Alnus glutionosa induced by herbivory are quantitatively related to the extent of damage. In: J. Chem. Ecol. 37 (2011), Nr. 1, S. 18-28

[69] Capitani, Donatella ; Brilli, Federico ; Mannina, Luisa ; Proietti, Noemi ; LORETO, Francesco: In situ investigation of leaf water status by portable unilateral nuclear magnetic resonance. In: Plant Physiol. 149 (2009), Nr. 4, S. 1638-1647

[70] Schütr, Peter (Hrsg.) ; Schuck, H J. (Hrsg.) ; Stimm, B (Hrsg.): Enzyklopädie der Laubbäume: Die große Enzyklopädie. Hamburg, DE: Nikol, 2006. - 581 S. ISBN 3-933203-53-8

[71] Polley, H ; Hennig, P ; Kroiher, F ; Marks, A ; Riedel, T ; Schmidt, U ; Schwitzgebel, F ; Stauber, T ; Bundesministerium für Ernährung und LandwirTschaft (Hrsg.): Der Wald in Deutschland: Ausgewählte Ergebnisse der dritten Bundeswaldinventur. 3. Aufl. Frankfurt am Main, DE: Druck- und Verlagshaus Zarbock, 2018. - 52 S.

[72] Knoke, Thomas ; Ammer, Christian ; Stimm, Bernd ; Mosande, Reinhard: Admixing broadleaved to coniferous tree species: a review on yield, ecological stability and economics. In: Eur. J. of For. Res. 127 (2008), Nr. 2, S. 89-101

[73] RÖHRIG, Ernst ; BARTsCH, Norbert: Der Wald als Vegetationsform und seine Bedeutung für den Menschen. 6. Aufl. Hamburg, DE: Paul Parey, 1992. - 350 S. ISBN 987-3-8001-4556-1

[74] Leuschner, Chr: Mechanismen der Konkurrenzüberlegenheit der Rotbuche. In: Berichte der Reinhold-Tüxen-Gesellschaft 10 (1998), S. 5-18

[75] Packham, John R. ; Thomas, Peter A. ; Atkinson, Mark D. ; Degen, Thomas: Biological flora of the British Isles: Fagus sylvatica. In: J. Ecol. 100 (2012), Nr. 6, S. $1557-1608$

[76] Leuschner, Christoph ; Meier, Ina C. ; Hertel, Dietrich: On the niche breadth of Fagus sylvatica: soil nutrient status in 50 Central European beech stands on a broad range of bedrock types. In: Ann. For. Sci. 63 (2006), Nr. 4, S. 355-368 
[77] Dittmar, Christoph ; Zech, Wolfgang ; Elling, Wolfram: Growth variations of common beech (Fagus sylvatica L.) under different climatic and environmental conditions in Europe - a dendroecological study. In: For. Ecol. Manage. 173 (2003), Nr. 1-3, S. 63-78

[78] Lebourgeois, F ; Bréda, N ; Ulrich, E ; Granier, A: Climate-tree-growth relationships of European beech (Fagus sylvatica L.) in the French Permanent Plot Network (RENECOFOR). In: Trees 19 (2005), Nr. 4, S. 385-401

[79] Meier, Ina C. ; Leuschner, Christoph: Belowground drought response of European beech: fine root biomass and carbon partitioning in 14 mature stands across a precipitation gradient. In: Glob. Chang. Biol. 14 (2008), Nr. 9, S. 2081-2095

[80] Eichhorn, Johannes ; Dammann, Inge ; Schönfelder, Egbert ; Albrecht, Mascha ; BECK, Wolfgang ; PAAR, Uwe: Untersuchungen zur Trockenheitstoleranz der Buche am Beispiel des witterungsextremen Jahres 2003. In: Ergebnisse angewandter Forschung zur Buche. NW-FVA, 2008. - ISBN 978-3-940344-44-1, S. $109-134$

[81] Leuschner, Christoph ; HerTel, Dietrich: Fine root biomass of temperate forests in relation to soil acidity and fertility, climate, age and species. In: Prog. Bot. 64 (2003), S. 405-438

[82] Rose, Laura ; Leuschner, Christoph ; Köckemann, Benjamin ; Buschmann, Holger: Are marginal beech (Fagus sylvatica L.) provenances a source for drought tolerant ecotypes? In: Eur. J. For. Res. 128 (2009), Nr. 4, S. 335-343

[83] Backes, K ; Leuschner, Ch: Leaf water relations of competitive Fagus sylvatica and Quercus petraea trees during 4 years differing in soil drought. In: Can. J. For. Res. 30 (2000), Nr. 3, S. 335-346

[84] Ciais, Ph ; Reichstein, M ; Viovy, Nicolas ; Granier, André ; OGée, Jérôme ; Allard, Vincent ; Aubinet, Marc ; Buchmann, N ; Bernhofer, Chr ; CarraRA, Arnaud ; Chevallier, F ; De Noblet, N ; Friend, A D. ; Friedlingstein, $\mathrm{P}$; Grünwald, T ; Heinesch, B ; Keronen, P ; Knohl, A ; Krinner, G ; Loustau, D ; Manca, G ; Matteucci, G ; Miglietta, F ; Ourcival, J M. ; Papale, D ; Pilegaard, K ; Rambal, S ; Seufert, G ; Soussana, J F. ; Sanz, M J. ; Schulze, E D. ; Vesala, T ; Valentini, R: Europe-wide reduction in primary productivity caused by the heat and drought in 2003. In: Nature 437 (2005), Nr. 7058, S. 529-533 
[85] Manthey, Michael ; Leuschner, Christoph ; Härdtle, Werner: Buchenwälder und Klimawandel. In: Natur und Landschaft 82 (2007), Nr. 9/10, S. 441-445

[86] Jump, Alistair S. ; Hunt, Jenny M. ; PeÑuelas, Josep: Rapid climate changerelated growth decline at the southern range edge of Fagus sylvatica. In: Glob. Chang. Biol. 12 (2006), Nr. 11, S. 2163-2174

[87] Petrakis, Panos V. ; Spanos, Kostas ; Feest, Alan ; Daskalakou, Evangelia: Phenols in leaves and bark of Fagus sylvatica as determinants of insect occurrences. In: Int. J. Mol. Sci. 12 (2011), Nr. 5, S. 2769-2782

[88] Walentowski, Helge ; Bu $\beta$ Ler, H ; Bergmeier, E ; Blaschke, M ; Finkeldey, R ; Gossner, M M. ; Litt, T ; Müller-Kroehling, S ; Philippi, G ; Pop, V V. ; Reif, A ; Schulze, E-D ; Strëtz, C ; Wirth, V: Sind die deutschen Waldnaturschutzkonzepte adäquat für die Erhaltung der buchenwaldtypischen Flora und Fauna? Eine kritische Bewertung basierend auf der Herkunft der Waldarten des mitteleuropäischen Tief- und Hügellandes. In: Forstarchiv 81 (2010), Nr. 5, S. 195217

[89] DelB, Horst: Rindenbrüter an Buche. In: FVA-Waldschutz-Info 4 (2004), S. 1-16

[90] FülDnER, Kai: Anmerkungen zur Biologie und zum Verhalten des Nagelflecks Aglia tau (Linnaeus, 1758)(Lepidoptera: Saturniidae). In: Nachr. entomol. Ver. Apollo, N.F. 20 (2000), Nr. 3/4, S. 311-319

[91] GorA, Vincenz ; KÖNIG, Jürgen ; LunderstÄDT, Jörg: Physiological defence reactions of young beech trees (Fagus sylvatica) to attack by Phyllaphis fagi. In: For. Ecol. Manage. 70 (1994), Nr. 1-3, S. 245-254

[92] NeF, Ludovic ; PERRIN, Robert: Damaging agents in European forest nurseries: practical handbook. Luxembourg, LUX: European Communities, 1999. - 352 S. ISBN 978-9-2828-2803-8

[93] Schmutterer, Heinrich: Zur Kenntnis der Buchenblattlaus Phyllaphis fagi (L.)(Homoptera, Aphidoidea), einer wichtigen Honigtauerzeugerin auf Buche. In: Anzeiger für Schädlingskunde 25 (1952), Nr. 1, S. 1-5

[94] Heiermann, Julian ; Schütz, Stefan: The effect of the tree species ratio of European beech (Fagus sylvatica L.) and Norway spruce (Picea abies (L.) Karst.) on polyphagous and monophagous pest species - Lymantria monacha L. and Calliteara pudibunda L.(Lepidoptera: Lymantriidae) as an example. In: For. Ecol. and Manage. 255 (2008), Nr. 3-4, S. 1161-1166 
[95] Urban, J ; Sedivy, J: Factors regulating the mass outbreak of the pale tussock moth (Calliteara pudibunda, Lepidoptera, Lymantriidae). In: Lesnictvi-UZPI (Czech Republic) 43 (1997), Nr. 2, S. 67-78

[96] Schwenke, Wolfgang: Die Forstschädlinge Europas. Bd. 3. Hamburg, DE: Paul Parey, 1978. - 467 S. - ISBN 3-490-11316-0

[97] Kölling, Christian ; Zimmermann, Lothar: Die Anfälligkeit der Wälder Deutschlands gegenüber dem Klimawandel. In: Gefahrstoffe-Reinhalt. Luft 67 (2007), Nr. 6, S. 259-268

[98] Hoch, GERNOT ; Putz, JASMIN ; Krehan, HANNES: Forstlicher Pflanzenschutz im globalen Wandel. In: BFW-Praxisinformation 44 (2017), S. 10-13

[99] Robinet, Christelle ; Roques, Alain: Direct impacts of recent climate warming on insect populations. In: Integr. Zool. 5 (2010), Nr. 2, S. 132-142

[100] Jönsson, Anna M. ; Harding, Susanne ; BÄrring, Lars ; Ravn, Hans P.: Impact of climate change on the population dynamics of Ips typographus in southern Sweden. In: Agric. For. Meteorol. 146 (2007), Nr. 1-2, S. 70-81

[101] Petercord, Ralf: Zukünftige Gefährdung der Rotbuche durch rinden- und holzbrütende Käfer in Baden-Württemberg. In: Mitt. Dtsch. Ges. Allg. Angew. Ent. 16 (2008), S. 247-250

[102] Volney, W Jan A. ; Fleming, Richard A.: Climate change and impacts of boreal forest insects. In: Agri. Ecosyst. Environ. 82 (2000), Nr. 1-3, S. 283-294

[103] Hoch, Gernot ; Cech, THOMAS ; Perny, BERnhARD: Die Profiteure vom Wald ohne Wasser: Warum Trockenheit Borkenkäfer, Triebsterbenspilze und andere Schadorganismen begünstigt. In: BFW-Praxisinformation 40 (2016), S. 9-11

[104] Knutzen, Florian ; Dulamsuren, Choimaa ; Meier, Ina C. ; Leuschner, Christoph: Recent climate warming-related growth decline impairs European beech in the center of its distribution range. In: Ecosystems 20 (2017), Nr. 8, S. 1494-1511

[105] Meier, Ina C. ; Knutzen, Florian ; Eder, Lucia M. ; Müller-Haubold, Hilmar ; Goebel, Marc-Oliver ; Bachmann, Jörg ; Hertel, Dietrich ; Leuschner, Christoph: The deep root system of Fagus sylvatica on sandy soil: structure and variation across a precipitation gradient. In: Ecosystems 21 (2018), Nr. 2, S. 280-296

[106] Kaltenböck, Karl: Chromatographie für Dummies. Weinheim, DE: Wiley-VCH, 2010. - 384 S. - ISBN 978-3-527-70530-6 
[107] Gabriel, Martin ; RACHOW, Christine: Einführung in die Gaschromatographie und die Massenspektroskopie mit anschließender Auswertungseinweisung. Vorlesungsskript, 2011

[108] Plenio, H. ; Fleckenstein, Christoph ; An Der Heiden, Markus: Grundlagen der Chromatographie. Vorlesungsskript, 2011

[109] GotTwald, Wolfgang: GC für Anwender. VCH, Weinheim, 1995. - 284 S. - ISBN 3-527-28681-0

[110] BRInkeRT, Tobias: Grundlagen der Gaschromatographie (GC), Institut für Spektrochemie und angewandte Spektroskopie (ISAS) Dortmund, Abschlussarbeit, 2005

[111] Köhler, Martin ; Mirabelli, Mario: Gaschromatographie (GC). Vorlesungsskript, 2015

[112] Yuan, Joshua S. ; Himanen, Sari J. ; Holopainen, Jarmo K. ; Chen, Feng ; STEWART JR, C N.: Smelling global climate change: mitigation of function for plant volatile organic compounds. In: Trends Ecol. Evol. 24 (2009), Nr. 6, S. 323-331

[113] De Moraes, Consuelo M. ; Lewis, W J. ; Paré, P W. ; Alborn, H T. ; TumLINSON, J H.: Herbivore-infested plants selectively attract parasitoids. In: Nature 393 (1998), Nr. 6685, S. 570-573

[114] Keeling, Christopher I. ; Bohlmann, Jörg: Genes, enzymes and chemicals of terpenoid diversity in the constitutive and induced defence of conifers against insects and pathogens. In: New Phytol. 170 (2006), Nr. 4, S. 657-675

[115] Joó, Éva ; Van Langenhove, Herman ; Šimpraga, M ; Steppe, Kathy ; AmeLynck, C ; Schoon, N ; MÜller, J-F ; Dewulf, Jo: Variation in biogenic volatile organic compound emission pattern of Fagus sylvatica L. due to aphid infection. In: Atmos. Environ. 44 (2010), Nr. 2, S. 227-234

[116] Pearse, Ian S. ; Hughes, Kathy ; Shiojiri, Kaori ; Ishizaki, Satomi ; Karban, Richard: Interplant volatile signaling in willows: revisiting the original talking trees. In: Oecologia 172 (2013), Nr. 3, S. 869-875

[117] StÄdler, E ; Roessingh, P: Perception of surface chemicals by feeding and ovipositing insects. In: Proceedings 7th International Symposium on Insect-Plant Relationships. Symposia Biologica Hungarica. 1991, S. 71-86 
[118] Bernays, E A. ; Chapman, R E.: Behavior: the process of host-plant selection. In: Host-plant selection by phytophagous insects. London, UK: Chapman and Hall, 1994. - ISBN 0-412-03131-0, S. 95-165

[119] Paczkowska, Marta ; RACHOw, Christine ; Schütz, Stefan: Electrophysiological reaction and behavioural response of the oligophagous moths Smerinthus ocellatus (L. 1758) and Cerura vinula (L. 1758) towards methyl salicylate and other host plant odors. In: Mitt. Dtsch. Ges. Allg. Angew. Ent. 18 (2012), S. 213-217

[120] Byers, J A.: Chemical ecology of bark beetles in a complex olfactory landscape. In: Lieutier, F (Hrsg.) ; Day, K R. (Hrsg.) ; Battisti, A (Hrsg.) ; GréGoire, Jean-Claude (Hrsg.) ; Evans, Hugh F. (Hrsg.): Bark and wood boring insects in living trees in Europe, a synthesis. Dordrecht, NL: Springer, 2007. - ISBN 978-14020-2240-1, S. 89-134

[121] Rachow, Christine ; Gabriel, Martin ; SchÜtz, Stefan: Olfactory response of Pale Tussock Calliteara pudibunda (L. 1758) (Lep., Noctuidae) and Tau Emperor Aglia tau (L. 1761) (Lep., Saturniidae) to stem volatiles of beech (Fagus sylvatica). In: Mitt. Dtsch. Ges. Allg. Angew. Ent. 19 (2014), S. 57-62

[122] Hupfeld, M ; Berendes, G ; Lehnardt, F: Buchenrotkern und Zielstärkennutzung. Untersucht im hessischen Forstamt Hatzfeld. In: AFZ DERWALD 52 (1997), S. $1024-1027$

[123] WeIS, Björn: Die radiale Diffusion totkernspezifischer Substanzen durch das Holz von Fagus sylvatica L., Universität Göttingen, Diplomarbeit

[124] Erlbeck, Reinhold ; Haseder, Ilse ; Stinglwagner, Gerhard: Kosmos Waldund Forstlexikon. 4. Aufl. Stuttgart, DE: Kosmos, 2009. - 1022 S. - ISBN 978-3440-12160-3

[125] Bressem, Ulrich: Komplexe Erkrankungen an Buche. Complex diseases in beech. In: NW-FVA (Hrsg.): Ergebnisse angewandter Forschung zur Buche. Bd. 3. Göttingen, DE: Universitätsverlag Göttingen, 2008. - ISBN 978-3-940344-44-1, S. $87-107$

[126] Holzke, C ; Dindorf, T ; Kesselmeier, J ; Kuhn, U ; Koppmann, R: Terpene emissions from European beech (shape Fagus sylvatica L.): pattern and emission behaviour over two vegetation periods. In: J. Atmos. Chem. 55 (2006), Nr. 1, S. 81-102 
[127] Tollsten, Lars ; MÜller, Peter M.: Volatile organic compounds emitted from beech leaves. In: Phytochemistry 43 (1996), Nr. 4, S. 759-762

[128] RutheR, J: Retention index database for identification of general green leaf volatiles in plants by coupled capillary gas chromatography-mass spectrometry. In: J. Chrom. A 890 (2000), Nr. 2, S. 313-319

[129] Rachow, Christine ; Gester-Gerstmann, Patrik ; Weis, Björn ; Schütz, Stefan: Ein neues Verfahren zum Sammeln von Stammvolatilen. In: Mitt. Dtsch. Ges. Allg. Angew. Ent. 18 (2012), S. 635-639

[130] König, Georg ; Brunda, Monika ; Puxbaum, Hans ; Hewitt, C N. ; Duckham, S C. ; Rudolph, Jochen: Relative contribution of oxygenated hydrocarbons to the total biogenic VOC emissions of selected mid-European agricultural and natural plant species. In: Atmos. Environ. 29 (1995), Nr. 8, S. 861-874

[131] Dindorf, T ; Kuhn, U ; Ganzeveld, L ; Schebeske, G ; Ciccioli, P ; Holzke, $\mathrm{C}$; Köble, R ; Seufert, G ; Kesselmeier, J: Emission of monoterpenes from European beech (Fagus sylvatica L.) as a function of light and temperature. In: Biogeosci. Discuss. (2005), Nr. 1, S. 137-182

[132] Šimpraga, M ; Verbeeck, Hans ; Demarcke, Marie ; Joó, Éva ; Amelynck, Crist ; Schoon, Niels ; Dewulf, Jo ; Van Langenhove, Herman ; Heinesch, Bernard ; Aubinet, Marc u. a.: Comparing monoterpenoid emissions and net photosynthesis of beech (Fagus sylvatica L.) in controlled and natural conditions. In: Atmospheric environment 45 (2011), Nr. 17, S. 2922-2928

[133] Demarcke, Marie ; Müller, J-F ; Schoon, Niels ; Van Langenhove, Herman ; Dewulf, Jo ; Joó, E ; Steppe, Kathy ; Šimpraga, M ; Heinesch, Bernard ; Aubinet, Marc ; Amelynck, C: History effect of light and temperature on monoterpenoid emissions from Fagus sylvatica L. In: Atmos. Environ. 44 (2010), Nr. 27, S. 3261-3268

[134] Flamini, G ; Cioni, P L. ; Morelli, I: Differences in the fragrances of pollen, leaves, and floral parts of Garland (Chrysanthemum coronarium) and composition of the essential oils from flowerheads and leaves. In: J. Agric. Food Chem. 51 (2003), Nr. 8, S. 2267-2271

[135] Keskitalo, Marjo ; Pehu, Eija ; Simon, James E.: Variation in volatile compounds from tansy (Tanacetum vulgare L.) related to genetic and morphological differences of genotypes. In: Biochem. Syst. Ecol. 29 (2001), Nr. 3, S. 267-285 
[136] Vrkočová, P ; Valterová, Irena ; Vrkoč, Jan ; Koutek, B: Volatiles released from oak, a host tree for the bark beetle Scolytus intricatus. In: Biochem. Syst. Ecol. 28 (2000), Nr. 10, S. 933-947

[137] Piechulla, B ; Pott, M B.: Plant scents - mediators of inter- and intraorganismic communication. In: Planta 217 (2003), Nr. 5, S. 687-689

[138] Schulze, Birgit ; Kost, Christian ; Arimura, Gen-Ichiro ; Boland, Wilhelm: Duftstoffe: Die Sprache der Pflanzen. Signalrezeption, Biosynthese und Ökologie. In: Chem. Unserer Zeit 40 (2006), Nr. 6, S. 366-377

[139] Schütz, Stefan ; Weissbecker, Bernhard ; Apel, Karl-Heinz ; Wenk, Matthias: Duftstoffe als Marker für die Befallsdisposition von Kiefern durch den Blauen Kiefernprachtkäfer Phaenops cyanea F. (Col., Buprestidae). In: Mitt. Dtsch. Ges. Allg. Angew. Ent. 14 (2004), S. 301-306

[140] Deutsches Institut FÜr Normung E. V.: Innenraumluftverunreinigungen Teil 6: Bestimmung in der Innenraumluft und in Prüfkammern, Probenahme auf Tenax $^{\circledR}$ TA, thermische Desorption und Gaschromatographie mit MS/FID (ISO 16000-6:2004). Berlin, DE: Beuth Verlag, 2004. - 31 S.

[141] Solomon, S ; Qin, D ; Manning, M ; Chen, Z ; Marquis, M ; Averyt, K ; Tignor, Melinda M-B ; L, Miller H.: Climate Change 200\%. The Physical Sience Basis. Contribution of working group I to the Fourth Assessment Report of the Intergovernmental Panel on Climate Change. Cambridge, UK: Cambridge University Press, 2007. - 996 S.

[142] Holighaus, Gerrit ; Schütz, Stefan: Odours of wood decay as semiochemicals for Trypodendron domesticum L.(Col., Scolytidae). In: Mitt. Dtsch. Ges. Allg. Angew. Ent. 15 (2006), S. 161-165

[143] Moukhtar, Sophie ; Bessagnet, Bertrand ; Rouil, Laurence ; Simon, V: Monoterpene emissions from Beech (Fagus sylvatica) in a French forest and impact on secondary pollutants formation at regional scale. In: Atmos. Environ. 39 (2005), Nr. 19, S. 3535-3547

[144] Schober, R (Hrsg.): Massentafeln zur Bestimmung des Holzgehaltes stehender Waldbäume and Wäldbestände. 10. Aufl. Berlin, DE: Paul Parey, 1952. - 216 S.

[145] Guenther, Alex ; Hewitt, C N. ; Erickson, David ; Fall, Ray ; Geron, Chris ; Graedel, Tom ; Harley, Peter ; Klinger, Lee ; Lerdau, Manuel ; McKay, 
W A. u. a.: A global model of natural volatile organic compound emissions. In: $J$. Geophys. Res. Atmos. 100 (1995), Nr. D5, S. 8873-8892

[146] Fehsenfeld, Fred ; Calvert, Jack ; Fall, Ray ; Goldan, Paul ; Guenther, Alex B. ; Hewitt, C N. ; Lamb, Brian ; Liu, Shaw ; Trainer, Michael ; WestBerG, Hal ; Zimmerman, Pat: Emissions of volatile organic compounds from vegetation and the implications for atmospheric chemistry. In: Glob. Biogeochem. Cycles 6 (1992), Nr. 4, S. 389-430

[147] Klein, D ; Schulz, C: Kohlenstoffspeicherung von Bäumen. In: LWF-Merkblatt (2011), Nr. 27, S. 1-6

[148] Claeys, Magda ; Graham, Bim ; Vas, Gyorgy ; Wang, Wu ; Vermeylen, Reinhilde ; Pashynska, Vlada ; Cafmeyer, Jan ; Guyon, Pascal ; Andreae, Meinrat O. ; ARTAXo, Paulo u.a.: Formation of secondary organic aerosols through photooxidation of isoprene. In: Science 303 (2004), Nr. 5661, S. 1173-1176

[149] Griffin, Robert J. ; Cocker III, David R. ; Seinfeld, John H. ; Dabdub, Donald: Estimate of global atmospheric organic aerosol from oxidation of biogenic hydrocarbons. In: Geophys. Res. Lett. 26 (1999), Nr. 17, S. 2721-2724

[150] Ceschia, Éric ; Damesin, Claire ; Lebaube, Stéphanie ; Pontailler, Jean-Yves ; DUfrêne, Éric: Spatial and seasonal variations in stem respiration of beech trees (Fagus sylvatica). In: Ann. For. Sci. 59 (2002), Nr. 8, S. 801-812

[151] Kováts, E: Gas-chromatographische Charakterisierung organischer Verbindungen. Teil 1: Retentionsindices aliphatischer Halogenide, Alkohole, Aldehyde und Ketone. In: Hel. Chim. Acta 41 (1958), Nr. 7, S. 1915-1932

[152] Schüler, Silvio ; Liesebach, Mirko ; von Wühlisch, Georg: Genetische Variation und Plastizität des Blattaustriebs von Herkünften der Rot-Buche. In: Appl. Agric. Forestry Res. 62 (2012), Nr. 4, S. 211-220

[153] Vitale, Marcello ; Salvatori, Elisabetta ; Loreto, Francesco ; Fares, Silvano ; MAnes, Fausto: Physiological responses of Quercus ilex leaves to water stress and acute ozone exposure under controlled conditions. In: Water Air Soil Pollut. 189 (2008), Nr. 1-4, S. 113-125

[154] Lindfors, Virpi ; Laurila, Tuomas: Biogenic volatile organic compound (VOC) emissions from forests in Finland. In: Boreal Environ. Res. 5 (2000), Nr. 2, S. 95-113 
[155] Rapparini, Francesca ; Baraldi, Rita ; FACini, Osvaldo: Seasonal variation of monoterpene emission from Malus domestica and Prunus avium. In: Phytochemistry 57 (2001), Nr. 5, S. 681-687

[156] Llusià, J ; Peñuelas, J: Changes in terpene content and emission in potted Mediterranean woody plants under severe drought. In: Can. J. Bot. 76 (1998), Nr. 8, S. $1366-1373$

[157] Kainulainen, P ; Oksanen, J ; Palomäki, V ; Holopainen, JK ; Holopainen, T: Effect of drought and waterlogging stress on needle monoterpenes of Picea abies. In: Can. J. Bot. 70 (1992), Nr. 8, S. 1613-1616

[158] Copolovici, Lucian ; Kännaste, Astrid ; Ninnemets, Ülo: Gas chromatographymass spectrometry method for determination of monoterpene and sesquiterpene emissions from stressed plants. In: Studia Universitatis Babes-Bolyai, Chemia 54 (2009), S. 329-339

[159] Khalid, Kh A.: Influence of water stress on growth, essential oil, and chemical composition of herbs (Ocimum sp.). In: Int. Agrophys. 20 (2006), Nr. 4, S. 289-296

[160] Schuh, G ; Heiden, A C. ; Hoffmann, Th ; Kahl, J ; Rockel, P ; Rudolph, $\mathrm{J}$; WiLDT, J: Emissions of volatile organic compounds from sunflower and beech: dependence on temperature and light intensity. In: J. Atmos. Chem. 27 (1997), Nr. 3, S. $291-318$

[161] Salerno, Gianandrea ; Frati, Francesca ; Marino, Giovanni ; Ederli, Luisa ; Pasqualini, Stefania ; Loreto, Francesco ; Colazza, Stefano ; Centritto, Mauro: Effects of water stress on emission of volatile organic compounds by Vicia faba, and consequences for attraction of the egg parasitoid Trissolcus basalis. In: $J$. Pest Sci. 90 (2017), Nr. 2, S. 635-647

[162] Fischbach, Robert J. ; Staudt, Michael ; Zimmer, Ina ; Rambal, Serge ; SCHNitzLER, Jörg-Peter: Seasonal pattern of monoterpene synthase activities in leaves of the evergreen tree Quercus ilex. In: Physiol. Plant. 114 (2002), Nr. 3, S. $354-360$

[163] Granier, A ; Biron, P ; Bréda, N ; Pontailler, J-Y ; Saugier, B: Transpiration of trees and forest stands: short and long-term monitoring using sapflow methods. In: Glob. Chang. Biol. 2 (1996), S. 265-274

[164] Hack, H ; Bleiholder, H ; Buhr, L ; Meier, U ; Schnock-Fricke, U u.a.: A uniform code for phenological growth stages of mono-and dicotyledonous plants. 
Extended BBCH scale, general-Einheitliche Codierung der phanologischen Entwicklungsstadien mono-und dikotyler Pflanzen. Erweiterte BBCH-Skala, Allgemein. In: Nachrbl. Deut. Pflanzenschutzd. 44 (1992), S. 265-270

[165] MeIER, Uwe: Growth stages of mono-and dicotyledonous plants. Blackwell Wissenschafts-Verlag, 1997. - 622 S. - ISBN 3-8263-3152-4

[166] Finn, GA ; Straszewski, AE ; Peterson, V: A general growth stage key for describing trees and woody plants. In: Ann. Appl. Biol. 151 (2007), Nr. 1, S. 127-131

[167] Schuster, C ; Estrella, Nicole ; Menzel, Annette: Shifting and extension of phenological periods with increasing temperature along elevational transects in southern B avaria. In: Plant Biol. 16 (2014), Nr. 2, S. 332-344

[168] Čufar, Katarina ; De Luis, Martin ; SAZ, Miguel A. ; ČrepinšEK, Zalika ; KajfeŽ-Bogataj, Lučka: Temporal shifts in leaf phenology of beech (Fagus sylvatica) depend on elevation. In: Trees 26 (2012), Nr. 4, S. 1091-1100

[169] Prislan, Peter ; GriČAr, Jožica ; Luis, Martin de ; Smith, Kevin T. ; Čufar, Katarina: Phenological variation in xylem and phloem formation in Fagus sylvatica from two contrasting sites. In: Agric. For. Meteorol. 180 (2013), S. 142-151

[170] Wen, Yuan ; Corre, Marife D. ; Rachow, Christine ; Chen, Lin ; Veldkamp, Edzo: Nitrous oxide emissions from stems of alder, beech and spruce in a temperate forest. In: Plant and Soil 420 (2017), Nr. 1-2, S. 423-434

[171] Bundesministerium FÜr Bildung und Forschung: MOPMModelling Oak Processionary Moth. - Online erhältlich unter www.fona.de/mediathek/pdf/Perspektive_Erde_2015_10.pdf; abgerufen am 10. Februar 2019

[172] Schäff, T ; Wolf, M ; Metzger, J ; Petercord, R: Monitoring der bodenlebenden Stadien des Waldmaikäfers in Unterfranken. In: Forstschutz Aktuell 57/58 (2013), S. 18-21

[173] Petercord, Ralf: Waldschutzsituation in Schutzgebieten - Folgerungen für eine nachhaltige Forstwirtschaft. Bedeutung des integrierten Pflanzenschutzes in Zeiten des Klimawandels. In: LWF aktuell 87 (2012), S. 54-57

[174] Ditengou, Franck A. ; Müller, Anna ; Rosenkranz, Maaria ; Felten, Judith ; Lasok, Hanna ; Van Doorn, Maja M. ; Legué, Valerie ; PAlme, Klaus ; Schnitzler, Jörg-Peter ; Polle, Andrea: Volatile signalling by sesquiterpenes 
from ectomycorrhizal fungi reprogrammes root architecture. In: Nat. Commun. 6 (2015), Nr. 6279, S. 1-9

[175] Wan, Mingguo ; Li, Guoqing ; Zhang, Jibin ; Jiang, Daohong ; Huang, HungChang: Effect of volatile substances of Streptomyces platensis F-1 on control of plant fungal diseases. In: Biol. Control 46 (2008), Nr. 3, S. 552-559

[176] Bais, Harsh P. ; Weir, Tiffany L. ; Perry, Laura G. ; Gilroy, Simon ; Vivanco, Jorge M.: The role of root exudates in rhizosphere interactions with plants and other organisms. In: Annu. Rev. Plant Biol. 57 (2006), S. 233-266

[177] Neeru, Narula ; Kothe, Erika ; Behl, Rishi K.: Role of root exudates in plantmicrobe interactions. In: J. Appl. Bot. Food Qual. 82 (2009), Nr. 2, S. 122-130

[178] Rasmann, Sergio ; Hiltpold, Ivan ; Ali, Jared: The role of root-produced volatile secondary metabolites in mediating soil interactions. In: Montanaro, Giuseppe (Hrsg.): Advances in selected plant physiology aspects. Rijeka, HR: InTech, 2012. ISBN 9798-953-51-0557-2, S. 269-290

[179] Delory, Benjamin M. ; Delaplace, Pierre ; Fauconnier, Marie-Laure ; DU JARDin, Patrick: Root-emitted volatile organic compounds: can they mediate belowground plant-plant interactions? In: Plant Soil 402 (2016), Nr. 1-2, S. 1-26

[180] Leuschner, Christoph ; Hertel, Dietrich ; Muhs, Annette ; Schmid, Iris: Feinwurzel-Bestandesmassen der Rotbuche an verschiedenen Standorten innerhalb ihrer ökologischen Amplitude in Nordwest- und Mitteldeutschland. In: Verh. Ges. Ökol. 28 (1998), S. 429-434

[181] Smith, Sally E. ; ReAD, David J.: Mycorrhizal symbiosis. 3. Aufl. London, UK: Academic press, 2008. - 800 S. - ISBN 978-0-12-370526-6

[182] Newsham, K K. ; Fitter, A H. ; Watkinson, A R.: Root pathogenic and arbuscular mycorrhizal fungi determine fecundity of asymptomatic plants in the field. In: J. Ecol. 82 (1994), Nr. 4, S. 805-814

[183] Lehto, Tarja ; ZwiAzeK, Janusz J.: Ectomycorrhizas and water relations of trees: a review. In: Mycorrhiza 21 (2011), Nr. 2, S. 71-90

[184] Harley, John L. ; Harley, E L.: A check-list of mycorrhiza in the British flora. In: New Phytol. 105 (1987), S. 1-102

[185] Nörr, R. ; Kottke, I. ; Blaschke, M.: Das unterirdische Geheimnis von Steinpilz und Trüffel. In: LWF aktuell 41 (2003), S. 26-28 
[186] Frey-Klett, P ; Garbaye, J ; TARkKA, M: The mycorrhiza helper bacteria revisited. In: New Phytol. 176 (2007), Nr. 1, S. 22-36

[187] Bonfante, Paola ; AncA, Iulia-Andra: Plants, mycorrhizal fungi, and bacteria: a network of interactions. In: Annu. Rev. Microbiol. 63 (2009), S. 363-383

[188] Peñuelas, J ; Asensio, D ; Tholl, D ; Wenke, K ; Rosenkranz, M ; PieChulla, B ; Schnitzler, J P.: Biogenic volatile emissions from the soil. In: Plant Cell Environ. 37 (2014), Nr. 8, S. 1866-1891

[189] Effmert, Uta ; Kalderás, Janine ; Warnke, René ; Piechulla, Birgit: Volatile mediated interactions between bacteria and fungi in the soil. In: Journal of chemical ecology 38 (2012), Nr. 6, S. 665-703

[190] Hayward, S ; Muncey, R J. ; James, A E. ; Halsall, Crispin J. ; Hewitt, C N.: Monoterpene emissions from soil in a Sitka spruce forest. In: Atmos. Environ. 35 (2001), Nr. 24, S. 4081-4087

[191] Janson, Robert W.: Monoterpene emissions from Scots pine and Norwegian spruce. In: J. Geophys. Res. Atmos. 98 (1993), Nr. D2, S. 2839-2850

[192] Girlanda, M ; Perotto, S ; Bonfante, P: Mycorrhizal fungi: their habitats and nutritional strategies. In: EsSER, Karl (Hrsg.): The Mycota. Environmental and microbial relationships. 2. Aufl. Berlin, Heidelberg, DE: Springer, 2007. ISBN 978-3-540-71839-0, S. 229-256

[193] DARrah, P R.: The rhizosphere and plant nutrition: a quantitative approach. In: Plant Soil 155 (1993), Nr. 1, S. 1-20

[194] Mendes, Rodrigo ; Garbeva, Paolina ; RaAijmakers, Jos M.: The rhizosphere microbiome: significance of plant beneficial, plant pathogenic, and human pathogenic microorganisms. In: FEMS Microbiol. Rev. 37 (2013), Nr. 5, S. 634-663

[195] Gregory, Peter J.: Roots, rhizosphere and soil: the route to a better understanding of soil science? In: Eur. J. Soil Sci. 57 (2006), Nr. 1, S. 2-12

[196] Brimecombe, Melissa J. ; De LeiJ, F A A M. ; Lynch, James M.: Rhizodeposition and microbial populations. In: Pinton, Roberto (Hrsg.) ; VARAnini, Zeno (Hrsg.) ; NANNIPIERI, Paolo (Hrsg.): The rhizosphere. Biochemistry and organic susbstances at the soil-plant interface. Boca Raton, Florida, USA: CRC Press, 2007. - ISBN 978-0-8493-3855-7, S. 73-109 
[197] Nannipieri, Paolo ; Ascher, Judith ; Ceccherini, Maria T. ; LAndi, Loretta ; Pietramellara, Giacomo ; Renella, Giancarlo ; Valori, Federico: Microbial diversity and microbial activity in the rhizosphere. In: Ci. Suelo 25 (2007), Nr. 1, S. 89-97

[198] Wenke, Katrin ; KAI, Marco ; Piechulla, Birgit: Belowground volatiles facilitate interactions between plant roots and soil organisms. In: Planta 231 (2010), Nr. 3, S. $499-506$

[199] Nardi, S ; Concheri, G ; Pizzeghello, D ; Sturaro, A ; Rella, R ; Parvoli, G: Soil organic matter mobilization by root exudates. In: Chemosphere 41 (2000), Nr. 5, S. 653-658

[200] Viles, A L. ; Reese, R N.: Allelopathic potential of Echinacea angustifolia DC. In: Environ. Exp. Bot. 36 (1996), Nr. 1, S. 39-43

[201] Sun, Xue-Guang ; TANG, Ming: Effect of arbuscular mycorrhizal fungi inoculation on root traits and root volatile organic compound emissions of Sorghum bicolor. In: S. Afr. J. Bot. 88 (2013), S. 373-379

[202] Rostás, Michael ; CRIPps, Michael G. ; SilCOCK, Patrick: Aboveground endophyte affects root volatile emission and host plant selection of a belowground insect. In: Oecologia 177 (2015), Nr. 2, S. 487-497

[203] Ballhorn, Daniel J. ; Kautz, Stefanie ; Heil, Martin: Distance and sex determine host plant choice by herbivorous beetles. In: PLoS ONE 8 (2013), Nr. 2, S. e55602

[204] De Moraes, Consuelo M. ; Mescher, Mark C. ; Tumlinson, James H.: Caterpillar-induced nocturnal plant volatiles repel conspecific females. In: Nature 410 (2001), Nr. 6828, S. 577-580

[205] Winter, Thorsten R. ; RostÁs, Michael: Nitrogen deficiency affects bottom-up cascade without disrupting indirect plant defense. In: J. Chem. Ecol. 36 (2010), Nr. 6, S. 642-651

[206] Laothawornkitkul, Jullada ; Taylor, Jane E. ; Paul, Nigel D. ; Hewitt, C N.: Biogenic volatile organic compounds in the Earth system. In: New Phytol. 183 (2009), Nr. 1, S. 27-51

[207] Johnson, Scott N. ; Nielsen, Uffe N.: Foraging in the dark-chemically mediated host plant location by belowground insect herbivores. In: J. Chem. Ecol. 38 (2012), Nr. 6, S. 604-614 
[208] Asensio, Dolores ; Peñuelas, Josep ; Ogaya, Romà ; Llusià, Joan: Seasonal soil VOC exchange rates in a Mediterranean holm oak forest and their responses to drought conditions. In: Atmos. Environ. 41 (2007), Nr. 11, S. 2456-2466

[209] Isidorov, Valery ; JdAnOvA, Maria: Volatile organic compounds from leaves litter. In: Chemosphere 48 (2002), Nr. 9, S. 975-979

[210] Rohloff, Jens: Volatiles from rhizomes of Rhodiola rosea L. In: Phytochemistry 59 (2002), Nr. 6, S. 655-661

[211] Lin, Chun ; Owen, Susan M. ; Peñuelas, Josep: Volatile organic compounds in the roots and rhizosphere of Pinus spp. In: Soil Biol. Biochem. 39 (2007), Nr. 4, S. 951-960

[212] Ens, Emilie J. ; Bremner, John B. ; French, Kris ; Korth, Jurgen: Identification of volatile compounds released by roots of an invasive plant, bitou bush (Chrysanthemoides monilifera spp. rotundata), and their inhibition of native seedling growth. In: Biol. Invasions 11 (2009), Nr. 2, S. 275-287

[213] Mcloughlin, Emma ; Rhodes, Angela H. ; Owen, Susan M. ; Semple, Kirk T.: Biogenic volatile organic compounds as a potential stimulator for organic contaminant degradation by soil microorganisms. In: Environ. Pollut. 157 (2009), Nr. 1, S. 86-94

[214] Paavolainen, Laura ; Kitunen, Veikko ; Smolander, Aino: Inhibition of nitrification in forest soil by monoterpenes. In: Plant Soil 205 (1998), Nr. 2, S. 147-154

[215] CoBB JR, F W.: Inhibitory effects of volatile oleoresin components on Fomes annosus and four Ceratocystis species. In: Phytopathology 58 (1968), S. 1327-1335

[216] Melin, ELIAS ; KrupA, SAGAR: Studies on ectomycorrhizae of pine II. Growth inhibition of mycorrhizal fungi by volatile organic constituents of Pinus silvestris (Scots Pine) roots. In: Physiol. Plant. 25 (1971), Nr. 3, S. 337-340

[217] Akiyama, Kohki ; Matsuzaki, Ken ; Hayashi, Hideo: Plant sesquiterpenes induce hyphal branching in arbuscular mycorrhizal fungi. In: Nature 435 (2005), Nr. 7043 , S. $824-827$

[218] Walter, Michael H. ; Hans, Joachim ; Strack, Dieter: Two distantly related genes encoding 1-deoxy-D-xylulose 5-phosphate synthases: differential regulation in shoots and apocarotenoid-accumulating mycorrhizal roots. In: The Plant Journal 31 (2002), Nr. 3, S. 243-254 
[219] Kleinheinz, G T. ; Bagley, S T. ; John, W P S. ; Rughani, J R. ; McGinnis, G D.: Characterization of alpha-pinene-degrading microorganisms and application to a bench-scale biofiltration system for VOC degradation. In: Arch. Environ. Contam. Toxicol. 37 (1999), Nr. 2, S. 151-157

[220] Steeghs, Marco ; Bais, Harsh P. ; Gouw, Joost de ; Goldan, Paul ; Kuster, William ; Northway, Megan ; Fall, Ray ; Vivanco, Jorge M.: Proton-transferreaction mass spectrometry as a new tool for real time analysis of root-secreted volatile organic compounds in Arabidopsis. In: Plant Physiol. 135 (2004), Nr. 1, S. 47-58

[221] Kalemba, D ; Kusewicz, D ; Świasder, K: Antimicrobial properties of the essential oil of Artemisia asiatica Nakai. In: Phytotherapy Research 16 (2002), Nr. 3, S. $288-291$

[222] Rasmann, Sergio ; Köllner, Tobias G. ; Degenhardt, Jörg ; Hiltpold, Ivan ; Toepfer, Stefan ; Kuhlmann, Ulrich ; Gershenzon, Jonathan ; Turlings, Ted C J.: Recruitment of entomopathogenic nematodes by insect-damaged maize roots. In: Nature 434 (2005), Nr. 7034, S. 732-737

[223] Hiltpold, Ivan ; Toepfer, Stefan ; Kuhlmann, Ulrich ; Turlings, Ted C J.: How maize root volatiles affect the efficacy of entomopathogenic nematodes in controlling the western corn rootworm? In: Chemoecology 20 (2010), Nr. 2, S. 155-162

[224] Weissteiner, S ; Schütz, S: Are different volatile pattern influencing host plant choice of belowground living insects? In: Mitt. Dtsch. Ges. Allg. Angew. Ent. 15 (2006), S. 51-55

[225] Robert, Christelle A M. ; Erb, Matthias ; Duployer, Marianne ; Zwahlen, Claudia ; Doyen, Gwladys R. ; Turlings, Ted C J.: Herbivore-induced plant volatiles mediate host selection by a root herbivore. In: New Phytol. 194 (2012), Nr. 4, S. 1061-1069

[226] Palma, R ; Mutis, A ; Manosalva, L ; Ceballos, R ; Quiroz, A: Behavioral and electrophysiological responses of Hylastinus obscurus to volatiles released from the roots of Trifolium pratense L. In: J. Soil Sci. Plant Nutr. 12 (2012), Nr. 1, S. 183-193

[227] Dudareva, Natalia ; Pichersky, Eran: Metabolic engineering of plant volatiles. In: Curr. Opin. Biotechnol. 19 (2008), Nr. 2, S. 181-189 
[228] Almenar, Eva ; Auras, Rafael ; Wharton, Phillip ; Rubino, Maria ; Harte, Bruce: Release of acetaldehyde from $\beta$-cyclodextrins inhibits postharvest decay fungi in vitro. In: J. Agric. Food Chem. 55 (2007), Nr. 17, S. 7205-7212

[229] Fernando, W G D. ; Ramarathnam, Rajesh ; Krishnamoorthy, Akkanas S. ; SAVCHUK, Sarah C.: Identification and use of potential bacterial organic antifungal volatiles in biocontrol. In: Soil Biol. Biochem. 37 (2005), Nr. 5, S. 955-964

[230] Chuankun, Xu ; Minghe, Mo ; Leming, Zhang ; Keqin, Zhang: Soil volatile fungistasis and volatile fungistatic compounds. In: Soil Biol. Biochem. 36 (2004), Nr. 12, S. 1997-2004

[231] Guerin, P M. ; Ryan, M F.: Relationship between root volatiles of some carrot cultivars and their resistance to the carrot fly, Psila rosae. In: Entomol. Exp. Appl. 36 (1984), Nr. 3, S. 217-224

[232] Wijesekera, R O B. ; Jayewardene, A L. ; Rajapakse, Lakshmi S.: Volatile constituents of leaf, stem and root oils of cinnamon (Cinnamomum zeylanicum). In: J. Sci. Food Agric. 25 (1974), Nr. 10, S. 1211-1220

[233] Kennedy, Alan I. ; Deans, Stanley G. ; Svoboda, Katerina P. ; Gray, Alexander I. ; Waterman, Peter G.: Volatile oils from normal and transformed root of Artemisia absinthium. In: Phytochemistry 32 (1993), Nr. 6, S. 1449-1451

[234] Bertoli, Alessandra ; Pistelli, Luisa ; Morelli, Ivano ; Fraternale, Daniele ; GiAmPeRI, Laura ; RICCI, Donata: Volatile constituents of different parts (roots, stems and leaves) of Smyrnium olusatrum L. In: Flavour Fragr. J. 19 (2004), Nr. 6, S. $522-525$

[235] Abraham, John ; Giacomuzzi, Valentino ; Angeli, Sergio: Root damage to apple plants by cockchafer larvae induces a change in volatile signals below- and above-ground. In: Entomol. Exp. Appl. 156 (2015), Nr. 3, S. 279-289

[236] Hertel, Dietrich ; Strecker, Tanja ; Müller-Haubold, Hilmar ; Leuschner, Christoph: Fine root biomass and dynamics in beech forests across a precipitation gradient - is optimal resource partitioning theory applicable to water-limited mature trees? In: J. Ecol. 101 (2013), Nr. 5, S. 1183-1200

[237] Santantonio, D ; Hermann, R K.: Standing crop, production, and turnover of fine roots on dry, moderate, and wet sites of mature Douglas-fir in western Oregon. In: Ann. Sci. for. 42 (1985), Nr. 2, S. 113-142 
[238] Leuschner, Christoph ; Hertel, Dietrich ; Schmid, Iris ; Koch, Oliver ; Muhs, Annette ; Hölscher, Dirk: Stand fine root biomass and fine root morphology in old-growth beech forests as a function of precipitation and soil fertility. In: Plant Soil 258 (2004), Nr. 1, S. 43-56

[239] Leuschner, Ch ; Backes, K ; Hertel, D ; Schipka, F ; Schmitt, U ; Terborg, $\mathrm{O}$; Runge, M: Drought responses at leaf, stem and fine root levels of competitive Fagus sylvatica L. and Quercus petraea (Matt.) Liebl. trees in dry and wet years. In: For. Ecol. Manage. 149 (2001), Nr. 1-3, S. 33-46

[240] Coleman, W E. ; Melton, Robert G. ; Slater, Robert W. ; Kopfler, Frederick C. ; Voto, Stephen J. ; Allen, Wendy K. ; Aurand, Theresa A.: Determination of organic contaminants by the Grob closed-loop-stripping technique. In: $J$. Am. Water Works Assoc. 73 (1981), Nr. 2, S. 119-125

[241] MÜller, Sigrid ; Van Der Merwe, Andre ; Schildknecht, Hermann ; Visser, Johann H.: An automated system for large-scale recovery of germination stimulants and other root exudates. In: Weed Sci. 41 (1993), Nr. 1, S. 138-143

[242] Marcetic, M ; Petroviç, S ; Milenkoviç, M ; Vujisiç, Lj ; Tesevic, V ; Niketic, M: Composition and antimicrobial activity of root essential oil of Balkan endemic species Eryngium palmatum. In: Chem. Nat. Compd. 49 (2014), Nr. 6, S. $1140-1142$

[243] Thakeow, Prodepran ; Weissbecker, Bernhard ; Schütz, Stefan: Volatile organic compounds emitted from fungal-rotting beech (Fagus sylvatica). In: Mitt. Dtsch. Ges. Allg. Angew. Ent. 15 (2006), S. 157-160

[244] Splivallo, Richard ; Ottonello, Simone ; Mello, Antonietta ; Karlovsky, Petr: Truffle volatiles: from chemical ecology to aroma biosynthesis. In: New Phytol. 189 (2011), Nr. 3, S. 688-699

[245] Ruiz-LozAno, JM ; AzCón, R: Hyphal contribution to water uptake in mycorrhizal plants as affected by the fungal species and water status. In: Physiol. Plant. 95 (1995), S. 472-478

[246] Pollierer, Melanie M. ; Dyckmans, Jens ; Scheu, Stefan ; Haubert, Dominique: Carbon flux through fungi and bacteria into the forest soil animal food web as indicated by compound-specific ${ }^{13} \mathrm{C}$ fatty acid analysis. In: Funct. Ecol. 26 (2012), Nr. 4, S. 978-990 
[247] Sponn, Marie ; Kuzyakov, Yakov: Distribution of microbial- and root-derived phosphatase activities in the rhizosphere depending on $\mathrm{P}$ availability and $\mathrm{C}$ allocation - Coupling soil zymography with ${ }^{14} \mathrm{C}$ imaging. In: Soil Biol. Biochem. 67 (2013), S. 106-113

[248] Bruce, Toby J A. ; Wadhams, Lester J. ; Woodcock, Christine M.: Insect host location: a volatile situation. In: Trends Plant Sci. 10 (2005), Nr. 6, S. 269-274

[249] Hetherington, Alistair M. ; Woodward, F I.: The role of stomata in sensing and driving environmental change. In: Nature 424 (2003), Nr. 6951, S. 901-908

[250] Willmer, Colin ; Fricker, Mark: Stomata. 2. Aufl. London, UK: Chapman and Hall, 1996. - 375 S. - ISBN 0-412-72500-2

[251] Holopainen, Jarmo K. ; Gershenzon, Jonathan: Multiple stress factors and the emission of plant VOCs. In: Trends Plant Sci. 15 (2010), Nr. 3, S. 176-184

[252] Braud, I ; Dantas-Antonino, A C. ; Vauclin, M ; Thony, J L. ; Ruelle, P: A simple soil-plant-atmosphere transfer model (SiSPAT) development and field verification. In: J. Hydrol. 166 (1995), Nr. 3-4, S. 213-250

[253] WeI, Jianing ; KAnG, Le: Roles of (Z)-3-hexenol in plant-insect interactions. In: Plant Signal. Behav. 6 (2011), Nr. 3, S. 369-371

[254] Kai, Marco ; Wenke, Katrin ; Piechulla, Birgit: Duftstoffe im Erdreich. Flüchtige Metabolite als Infochemikalien. In: Biol. Unserer Zeit 39 (2009), Nr. 5, S. 313-319

[255] Bertin, Cecile; Yang, Xiaohan ; Weston, Leslie A.: The role of root exudates and allelochemicals in the rhizosphere. In: Plant Soil 256 (2003), Nr. 1, S. 67-83

[256] Hartge, Karl H. ; Horn, Rainer ; Bachmann, Jörg ; Peth, Stephan: Einführung in die Bodenphysik. 4. Aufl. Stuttgart, DE: Schweizerbart Sience Publishers, 2014. - 372 S. - ISBN 978-3-510-65280-8

[257] Ruiz, Joaquín ; Bilbao, Rafael ; Murillo, María B: Adsorption of different VOC onto soil minerals from gas phase: influence of mineral, type of VOC, and air humidity. In: Environ. Sci. Technol. 32 (1998), Nr. 8, S. 1079-1084

[258] Diamadopoulos, Es ; Sakellariadis, D ; Koukouraki, E: The effect of humic acid on the transport of volatile chlorinated hydrocarbons in soil. In: Water Res. 32 (1998), Nr. 11, S. 3325-3330 
[259] Atkins, Peter W. ; Jones, Loretta: Chemie - einfach alles. 2. Aufl. Weinheim, DE: Wiley-VCH, 2006. - 991 S. - ISBN 978-3-527-31579-6

[260] Daunderer, Max: Gifte im Alltag: Wo sie vorkommen, wie sie wirken, wie man sich dagegen schützt. 3. Aufl. Münschen, DE: C.H. Beck, 2011. - 288 S. - ISBN 978-3-406-61734-8

[261] Fahed, Layal ; Khoury, Madona ; Stien, Didier ; Ouaini, Naïm ; Eparvier, Véronique ; El Beyrouthy, Marc: Essential oils composition and antimicrobial activity of six conifers harvested in Lebanon. In: Chem. Biodivers. 14 (2017), Nr. 2, S. e1600235

[262] Manter, Daniel K. ; Kelsey, Rick G. ; Karchesy, Joseph J.: Antimicrobial activity of extractable conifer heartwood compounds toward Phytophthora ramorum. In: J. Chem. Ecol. 33 (2007), Nr. 11, S. 2133-2147

[263] Mortimer, Charles E. ; MÜller, Ulrich: Chemie: Das Basiswissen der Chemie; 126 Tabellen. 9. Aufl. Stuttgart, DE: Georg Thieme Verlag, 2007. - 784 S. - ISBN 978-3-134-84309-5

[264] UHDE, Erik: Vergleich von Emissionsprüfkammern: Untersuchungen unter dynamischen Bedingungen, Technische Universität Carolo-Wilhelmina, Dissertation, 1998

[265] Mersmann, Alfons: Berechnen von Diffusionskoeffizienten. In: Stoffübertragung. Berlin, Heidelberg, DE: Springer, 1986. - ISBN 978-3-642-82617-7, S. 195-207

[266] Baehr, Hans D. ; Stephan, Karl: Wärme- und Stoffübertragung. 8. Aufl. Springer, Berlin, Heidelberg, 2013. - 804 S. - ISBN 978-3-642-36558-4

[267] Hiltpold, Ivan ; Turlings, Ted C J.: Belowground chemical signaling in maize: when simplicity rhymes with efficiency. In: J. Chem. Ecol. 34 (2008), Nr. 5, S. 628-635

[268] SzEwZyK, Ulrich ; SzEwzyK, Regine: Biofilme - die etwas andere Lebensweise. In: BIOspektrum 9 (1986), Nr. 3, S. 253-255

[269] Hiltpold, Ivan ; Bernklau, Elisa ; Bjostad, Louis B. ; Alvarez, Nadir ; Miller-Struttmann, Nicole E. ; Lundgren, Jonathan G. ; HibBard, Bruce E.: Nature, evolution and characterisation of rhizospheric chemical exudates affecting root herbivores. In: Johnson, Sccott (Hrsg.) ; Hiltpold, Ivan (Hrsg.) ; TuRLINGS, Ted C J. (Hrsg.): Advances in insect physiology. Behaviour an physiology of root herbivores Bd. 45. Elsevier, 2013. - ISBN 978-0-12-417165-7, S. 97-157 
[270] Payne, D ; Gregory, PJ: The soil atmosphere. In: Wild, A (Hrsg.): Russell's Soil Conditions and Plant Growth. 11. Aufl. Harlow, Essex, UK: Longman Scientific and Technical, 1988, S. 298-314

[271] Bundesministerium FÜr Verbraucherschutz, ERnährung Und LANDWIRTschaft: Die zweite Bundeswaldinventur - BWI II. Das Wichtigste in Kürze. Bonn, 2004. - 87 S.

[272] Ebert, G (Hrsg.): Die Schmetterlinge Baden-Württembergs, Bd. 4: Nachtfalter II. 3. Aufl. Stuttgart, DE: Eugen Ulmer Verlag, 1994. - 535 S. - ISBN 3-8001-3474-8

[273] Wei $\beta$ Becker, B ; Holighaus, G ; Schütz, S: Gas chromatography with mass spectrometric and electroantennographic detection: analysis of wood odorants by direct coupling of insect olfaction and mass spectrometry. In: J. Chrom. A 1056 (2004), Nr. 1-2, S. 209-216

[274] Schütz, S ; Wei $\beta$ Becker, B ; Koch, U T. ; Hummel, H E.: Detection of volatiles released by diseased potato tubers using a biosensor on the basis of intact insect antennae. In: Biosens. Bioelectron. 14 (1999), Nr. 2, S. 221-228

[275] Kaissling, K-E ; Thorson, J: Insect olfactory sensilla: structural, chemical and electrical aspects of the functional organisation. In: SATElle, D B. (Hrsg.) ; Hall, L M. (Hrsg.) ; Hildebrand, J G. (Hrsg.): Receptors for neurotransmitters, hormones and pheromone in insects. Amsterdam, NL: Elsevier, 1980, S. 261-282

[276] Coats, J R. ; Karr, L L. ; Drewes, C D.: Toxicity and neurotoxic effects of monoterpenoids in insects and earthworms. In: Hedin, P A. (Hrsg.): Naturally occuring pest bioregulators. Washington, USA: American Chemical Society, 1991. ISBN 0-8412-1897-8, S. 305-316

[277] Schopfer, Peter ; Brennicke, Axel: Pflanzenphysiologie. 7. Aufl. Heidelberg, DE: Spektrum Akademischer Verlag, 2010. - 702 S. - ISBN 978-3-8274-2351-1

[278] Fujimori, T ; Kasuga, R ; Kaneko, H ; Noguchi, M: Neutral volatile components of Burley tobacco. In: Beiträge zur Tabakforschung International 9 (1978), S. $317-325$

[279] Kappers, I F. ; Aharoni, Asaph ; Herpen, Teun W J M. ; Luckerhoff, Ludo L P. ; Dicke, Marcel ; Bouwmeester, H J.: Genetic engineering of terpenoid metabolism attracts bodyguards to Arabidopsis. In: Sience 309 (2005), Nr. 5743, S. 2070-2072 
[280] Mazzoglio, Peter J. ; Paoletta, Massimo ; Patetta, Augusto ; Currado, Italo: Calliteara pudibunda (Lepidoptera Lymantriidae) in Northwest Italy. In: Bull. Insectology 58 (2005), Nr. 1, S. 25-34

[281] Lerdau, M ; Dilts, S ; Westberg, H ; Lamb, B ; Allwine, G: Monoterpene emission from Ponderosa pine. In: J. Geophys. Res. 99 (1994), S. 16609-16615

[282] Loreto, Francesco ; Ciccioli, Paolo ; Cecinato, Angelo ; Brancaleoni, Enzo ; Frattoni, Massimiliano ; Tricoli, Domenico: Influence of environmental factors and air composition on the emission of [alpha]-pinene from Quercus ilex leaves. In: Plant Physiol. 110 (1996), Nr. 1, S. 267-275

[283] Bertin, N ; StaudT, M: Effect of water stress on monoterpene emissions from young potted holm oak (Quercus ilex L.) trees. In: Oecologia 107 (1996), Nr. 4, S. $456-462$

[284] Scharnweber, Tobias ; Manthey, Michael ; Criegee, Christian ; Bauwe, Andreas ; Schröder, Christian ; Wilmking, Martin: Drought matters - Declining precipitation influences growth of Fagus sylvatica L. and Quercus robur L. in northeastern Germany. In: For. Ecol. Manage. 262 (2011), Nr. 6, S. 947-961

[285] Weber, Pascale ; Heiri, Caroline ; Lévesque, Mathieu ; Sanders, Tanja ; Trotsiuk, Volodymyr ; WALtherT, Lorenz: Zuwachs und Klimasensitivität von Baumarten im Ökogramm der kollinen und submontanen Stufe. In: Schweiz. Z. Forstwes. 166 (2015), Nr. 6, S. 380-388

[286] Pichersky, Eran ; Raguso, Robert A.: Why do plants produce so many terpenoid compounds? In: New Phytol. 220 (2018), Nr. 3, S. 692-702

[287] Heldt, Hans W. ; Piechulla, Birgit: Eine große Vielfalt von Isoprenoiden erfüllt sehr unterschiedliche Funktionen im Pflanzenstoffwechsel. In: HELDT, Hans W. (Hrsg.) ; Piechulla, Birgit (Hrsg.): Pflanzenbiochemie. 5. Aufl. Heidelberg, Berlin, DE: Springer, 2015. - ISBN 978-3-662-44398-9, S. 393-413

[288] TAYlor, W E. ; ViCKERY, B: Insecticidal properties of limonene, a constituent of citrus oil. In: Ghana J. Agri. Sci. 7 (1974), S. 61-62

[289] Reid, Mary L. ; Purcell, J R C.: Condition-dependent tolerance of monoterpenes in an insect herbivore. In: Arthropod Plant Interact. 5 (2011), Nr. 4, S. 331-337

[290] Gouinguené, Sandrine ; Pickett, John A. ; Wadhams, Lester J. ; Birkett, Michael A. ; TurLings, Ted C J.: Antennal electrophysiological responses of three 
parasitic wasps to caterpillar-induced volatiles from maize (Zea mays mays), cotton (Gossypium herbaceum), and cowpea (Vigna unguiculata). In: J. Chem. Ecol. 31 (2005), Nr. 5, S. 1023-1038

[291] Williams, Livy ; Rodriguez-Saona, Cesar ; Castle, Sandra C. ; Zhu, Su: EAG-active herbivore-induced plant volatiles modify behavioral responses and host attack by an egg parasitoid. In: J. Chem. Ecol. 34 (2008), Nr. 9, S. 1190-1201

[292] WeI, Jian-Rong ; Lu, Xi-Ping ; JiAng, Li: Monoterpenes from larval frass of two Cerambycids as chemical cues for a parasitoid, Dastarcus helophoroides. In: Journal of insect science 13 (2013), Nr. 1, S. 1-12

[293] Peterson, Joseph K. ; Horvat, Robert J. ; Elsey, Kent D.: Squash leaf glandular trichome volatiles: identification and influence on behavior of female pickleworm moth [Diaphania nitidalis (Stoll.)](Lepidoptera: Pyralidae). In: J. Chem. Ecol. 20 (1994), Nr. 8, S. 2099-2109

[294] Keuneke, Karen ; KÖHLER, Gabriele: Strategien zur Regulierung von im Gemüsebau schädigenden Blumenfliegenarten (Anthomyiidae): Wurzelfliegen an Buschbohnen (Delia platura, Delia florilega), Zwiebelfliege (Delia antiqua), Kleine Kohlfliege (Delia radicum), Sächsische Landesanstalt für Landwirtschaft, Abschlussbericht, 2003

[295] Gugerli, Felix ; Frank, Aline ; Rellstab, Christian ; Pluess, Andrea R. ; MoSer, Barbara ; Arend, Matthias ; Sperisen, Christoph ; Wohlgemuth, Thomas ; HeIrI, Caroline: 3.2 Genetische Variation und lokale Anpassung bei Waldbaumarten im Zeichen des Klimawandels. In: Pleuss, Andrea R. (Hrsg.) ; Augustin, S (Hrsg.) ; Brang, P (Hrsg.): Wald im Klimawandel. Grundlagen für Adaptationsstrategien. 2016, S. 93-103

[296] Buiteveld, J ; Vendramin, GG ; Leonardi, S ; Kamer, K ; Geburek, Th: Genetic diversity and differentiation in European beech (Fagus sylvatica L.) stands varying in management history. In: For. Ecol. Manage. 247 (2007), Nr. 1-3, S. 98-106

[297] JumP, Alistair S. ; Penuelas, Josep: Extensive spatial genetic structure revealed by AFLP but not SSR molecular markers in the wind-pollinated tree, Fagus sylvatica. In: Mol. Ecol. 16 (2007), Nr. 5, S. 925-936

[298] Eller, Allyson S. ; De Gouw, Joost ; Graus, Martin ; Monson, Russell K.: 
Variation among different genotypes of hybrid poplar with regard to leaf volatile organic compound emissions. In: Ecol. Appl. 22 (2012), Nr. 7, S. 1865-1875

[299] I $\beta$ LEIB, Dörte: Genetische Strukturen in Buchen-Altbeständen und Naturverjüngung (Fagus sylvatica L.) des Mittleren Erzgebirges. Ulmer, 2006. - 136 S. - ISBN 38001-5437-4

[300] Carsjens, Caroline ; NGuyen NGoc, Quynh ; Guzy, Jonas ; Knutzen, Florian ; Meier, Ina C. ; MÜller, Markus ; Finkeldey, Reiner ; Leuschner, Christoph ; POLLE, Andrea: Intra-specific variations in expression of stress-related genes in beech progenies are stronger than drought-induced responses. In: Tree Physiol. 34 (2014), Nr. 12, S. 1348-1361

[301] DWD: Climate Data Center (CDC): Historical monthly precipitation observations for Germany, version v007, 2018

[302] DiCKE, Marcel: Are herbivore-induced plant volatiles reliable indicators of herbivore identity to foraging carnivorous arthropods? In: Entomol. Exp. Appl. 91 (1999), S. $131-142$

[303] WeIS, Björn: Die radiale Diffusion rotkernspezifischer Substanzen durch das Holz von Fagus sylvatica L., Georg-August-Universität Göttingen, Masterarbeit, 2009

[304] CAdmic GmbH: ProtectFOREST. - Online erhältlich unter www.cadmic.de/protectforest.html; abgerufen am 10. Dezember 2019

[305] Pobkrut, Theerapat; Kerdcharoen, Teerakiat: Soil sensing survey robots based on electronic nose. In: 201414 th International Conference on Control, Automation and Systems (ICCAS 2014) IEEE, 2014. - ISBN 2093-7121, S. 1604-1609 


\section{A. Anhang}

\section{A.1. Laub-VOC von F. sylvatica}

Table A.1: Bekannte VOC von Zweigen und Blätter von F. sylvatica in der Literatur

\begin{tabular}{|c|c|c|c|c|}
\hline Stoffgruppe & VOC & LRI & Untersuchungsobjekt & Literatur \\
\hline MT & allgemein & & $\begin{array}{l}\text { Zweig, junge Pflanze } \\
\text { Zweig, adulter Baum }\end{array}$ & $\begin{array}{c}1,11,12] \\
{[7]}\end{array}$ \\
\hline MT & Tricyclen & 918 & Sonnenblätter & {$[5]$} \\
\hline MT & Thujen & 920 & Zweig & {$[10]$} \\
\hline MT & $\alpha$-Thujen & 924 & $\begin{array}{c}\text { Sonnenblätter } \\
\text { Zweig, junge Pflanze } \\
\text { Zweig, adulter Baum }\end{array}$ & $\begin{array}{c}{[3]} \\
{[4]} \\
{[7,13]}\end{array}$ \\
\hline MT & $\beta$-Thujen & 926 & Zweig, junge Pflanze & {$[4]$} \\
\hline MT & $\alpha$-Pinen & 937 & $\begin{array}{c}\text { Sonnenblätter } \\
\text { Zweig } \\
\text { Zweig, junge Pflanze } \\
\text { Zweig, adulter Baum }\end{array}$ & $\begin{array}{c}{[3]} \\
{[10]} \\
{[4,6]} \\
{[5,7,8,13]}\end{array}$ \\
\hline MT & $\beta$-Pinen & 943 & $\begin{array}{c}\text { Sonnenblätter } \\
\text { Zweig, adulter Baum }\end{array}$ & $\begin{array}{c}{[3]} \\
{[2,5,7,8,13]}\end{array}$ \\
\hline MT & Camphen & 952 & $\begin{array}{c}\text { Sonnenblätter } \\
\text { Zweig, adulter Baum }\end{array}$ & $\begin{array}{l}3] \\
{[2]}\end{array}$ \\
\hline MT & Sabinen & 964 & $\begin{array}{c}\text { Sonnenblätter } \\
\text { Zweige } \\
\text { Zweig, junge Pflanze } \\
\text { Zweig, adulter Baum }\end{array}$ & $\begin{array}{c}{[3]} \\
{[10]} \\
{[4,6]} \\
{[2,5,7,8,13]}\end{array}$ \\
\hline MT & Myrcen & 982 & $\begin{array}{c}\text { Sonnenblätter } \\
\text { Zweig, adulter Baum }\end{array}$ & $\begin{array}{c}{[3]} \\
{[2,5,13]}\end{array}$ \\
\hline MT & $\beta$-Myrcen & 990 & Zweig, adulter Baum & {$[4]$} \\
\hline MT & $\alpha$-Phellandren & 997 & Sonnenblätter & {$[3]$} \\
\hline MT & (E)- $\beta$-Ocimen & 1001 & Zweig, junge Pflanze & {$[4]$} \\
\hline MT & $\alpha$-Terpinen & 1010 & $\begin{array}{c}\text { Sonnenblätter } \\
\text { Zweig, junge Pflanze } \\
\text { Zweig, adulter Baum }\end{array}$ & $\begin{array}{c}{[3]} \\
{[4]} \\
{[2,5,13]}\end{array}$ \\
\hline MT & p-Cymen & 1012 & $\begin{array}{c}\text { Sonnenblätter } \\
\text { Zweig, adulter Baum }\end{array}$ & $\begin{array}{c}{[3]} \\
{[2,5,13]}\end{array}$ \\
\hline MT & 3-Caren & 1014 & $\begin{array}{c}\text { Sonnenblätter } \\
\text { Zweig, adulter Baum }\end{array}$ & $\begin{array}{c}{[3]} \\
{[2,5]}\end{array}$ \\
\hline MT & $\beta$-Phellandren & 1021 & $\begin{array}{c}\text { Sonnenblätter } \\
\text { Zweig, adulter Baum }\end{array}$ & $\begin{array}{c}{[3]} \\
{[5,7,13]}\end{array}$ \\
\hline MT & (Z)-Ocimen & 1024 & Zweig, adulter Baum & {$[5]$} \\
\hline MT & (E)-Ocimen & 1034 & Zweig, adulter Baum & {$[13]$} \\
\hline MT & Limonen & 1034 & $\begin{array}{c}\text { Sonnenblätter } \\
\text { Zweig, junge Pflanze } \\
\text { Zweig, adulter Baum }\end{array}$ & $\begin{array}{c}{[3]} \\
{[6]} \\
{[2,5,7,8,13]}\end{array}$ \\
\hline MT & $\gamma$-Terpinen & 1049 & $\begin{array}{c}\text { Sonnenblätter } \\
\text { Zweig, junge Pflanze } \\
\text { Zweig, adulter Baum }\end{array}$ & $\begin{array}{c}{[3]} \\
{[4]} \\
{[2,5,7,13]}\end{array}$ \\
\hline MT & (E)-Sabinen hydrat & 1072 & Zweig, adulter Baum & {$[13]$} \\
\hline MT & $\alpha$-Terpinolen & 1078 & Zweig, junge Pflanze & {$[4]$} \\
\hline MT & Terpinolen & 1079 & $\begin{array}{c}\text { Sonnenblätter } \\
\text { Zweig, adulter Baum }\end{array}$ & $\begin{array}{c}{[3]} \\
{[7,13]}\end{array}$ \\
\hline
\end{tabular}




\begin{tabular}{|c|c|c|c|c|}
\hline Stoffgruppe & VOC & LRI & Untersuchungsobjekt & Literatur \\
\hline MT & Linalool & 1081 & $\begin{array}{l}\text { Zweig, junge Pflanze } \\
\text { Zweig, adulter Baum }\end{array}$ & $\begin{array}{c}4] \\
{[13]}\end{array}$ \\
\hline Homoterpen & DMNT & 1114 & $\begin{array}{l}\text { Zweig, junge Pflanze } \\
\text { Zweig, adulter Baum }\end{array}$ & $\begin{array}{c}{[4]} \\
{[13]}\end{array}$ \\
\hline ST & $\beta$-Bourbonen & 1386 & Zweig, junge Pflanze & {$[4]$} \\
\hline ST & Caryophyllen & 1424 & $\begin{array}{l}\text { Zweig, junge Pflanze } \\
\text { Zweig, adulter Baum }\end{array}$ & $\begin{array}{c}{[4]} \\
{[13]}\end{array}$ \\
\hline ST & $\alpha$-Humulen & 1456 & Zweig, junge Pflanze & {$[4]$} \\
\hline ST & Germacren D & 1481 & $\begin{array}{l}\text { Zweig, junge Pflanze } \\
\text { Zweig, adulter Baum }\end{array}$ & $\begin{array}{c}{[4]} \\
{[13]}\end{array}$ \\
\hline ST & $(\mathrm{Z})(\mathrm{E})-\alpha$-Farnesen & 1486 & $\begin{array}{l}\text { Zweig, junge Pflanze } \\
\text { Zweig, adulter Baum }\end{array}$ & $\begin{array}{c}{[4]} \\
{[13]}\end{array}$ \\
\hline ST & $(\mathrm{E})(\mathrm{E})-\alpha$-Farnesen & 1486 & $\begin{array}{l}\text { Zweig, junge Pflanze } \\
\text { Zweig, adulter Baum }\end{array}$ & $\begin{array}{c}{[4]} \\
{[13]}\end{array}$ \\
\hline Alken & Isopren & 504 & Zweig, adulter Baum & {$[7]$} \\
\hline Aldehyd & Hexanal & 805 & Zweig, adulter Baum & {$[7]$} \\
\hline GLV & (Z)-3-Hexen-1-ol & 858 & $\begin{array}{c}\text { Zweig } \\
\text { Zweig, adulter Baum }\end{array}$ & $\begin{array}{c}{[9]} \\
{[13]}\end{array}$ \\
\hline GLV & (Z)-3-Hexenylacetat & 987 & $\begin{array}{c}\text { Zweig } \\
\text { Zweig, junge Pflanze } \\
\text { Zweig, adulter Baum }\end{array}$ & $\begin{array}{c}9] \\
{[4]} \\
{[7,13]}\end{array}$ \\
\hline Aromat & Methylsalicylat & 1164 & Zweig, junge Pflanze & {$[4]$} \\
\hline Alkohol & 4-Terpineol & 1177 & Zweig, junge Pflanze & {$[4]$} \\
\hline
\end{tabular}

$\mathrm{LRI}=$ linearer Retentionsindex (Literatur), VOC = Flüchtige organische Verbindung (volatile organic compounds), MT = Monoterpen, $\mathrm{ST}=$ Sequiterpen, GLV = Grünblattdüfte (green leaf volatiles), junge Pflanze $=3$-6-jährige Pflanze, adulter Baum $=$ Waldbaum $(>15 \mathrm{~m})$

\section{Literatur:}

[1] Demarcke, M.; Müller, J.-F.,; Schoon, N.; Van Langenhove, H.; Dewulf, J.; Joó, É.; Steppe, K.; Šimpraga, B.; Heinesch, B.; Aubinet, M.; Amelynck, C.: History effect of light and temperature on monoterpenoid emissions from Fagus sylvatica L. In: Atmos. Environ. 44 (2010), Nr. 27, S. 3261-3268

[2] Dindorf, T.; Kuhn, U.; Ganzeveld, L.; Schebeske, G.; Ciccioli, P.; Holzke, C.; Köble, R.; Seufert, G.; Kesselmeier, J.: Emission of monoterpenes from European beech (Fagus sylvatica L.) as a function of light and temperature. In: Biogeosci. Discuss. 2 (2005), Nr. 1, S. 137-182

[3] Dindorf, T.; Kuhn, U.; Ganzeveld, L.; Schebeske, G.; Ciccioli, P.; Holzke, C.; Köble, R.; Seufert, G.; Kesselmeier, J.: Significant light and temperature dependent monoterpene emissions from European beech (Fagus sylvatica L.) and their potential impact on the European volatile organic compound budget. In: J. of Geophys. Res. 111 (2006), S. D16305

[4] Gossner, M. M.; Weisser, W. W.; Gershenzon, J.; Unsicker, S. B.: Insect attraction to herbivore-induced beech volatiles under different forest management regimes. In: Oecologia 176 (2014), Nr. 2, S. 569-580

[5] Holzke, C.; Dindorf, T.; Kesselmeier, J.; Kuhn, U.; Koppmann, R.: Terpene emissions from European beech (shape Fagus sylvatica L.): Pattern and Emission Behaviour Over two Vegetation Periods. In: J. of Atmos. Chem. 55 (2006), Nr. 1, S. 81-102

[6] Kahl, J.; Hoffmann, T.; Klockow, D.: Differentiation between de novo synthesized and con- 
stitutively released terpenoids from Fagus sylvatica. In: Phytochemistry 51 (1999), Nr. 3, S. 383-388

[7] König, G.; Brunda, M.; Puxbaum, H.; Hewitt, C. N.; Duckham, S. C.; Rudolph, J.: Relative contribution of oxygenated hydrocarbons to the total biogenic VOC emissions of selected mid-European agricultural and natural plant species. In: Atmos. Environ. 29 (1995), Nr. 8, S. 861-874

[8] Moukhtar, S.; Bessagnet, B.; Rouil, L.; Simon, V.: Monoterpene emissions from beech (Fagus sylvatica) in a French forest and impact on secondary pollutants formation at regional scale. In: Atmos. Environ. 39 (2005), Nr. 19, S. 3535-3547

[9] Ruther, J.: Retention index database for identification of general green leaf volatiles in plants by coupled capillary gas chromatography-mass spectrometry. In: J. Chrom. A 890 (2000), Nr. 2, S. 313-319

[10] Schuh, G.; Heiden, A. C.; Hoffmann, T.; Kahl, J.; Rockel, P.; Rudolph, J.; Wildt, J.: Emissions of volatile organic compounds from sunflower and beech: dependence on temperature and light intensity. In: J. Atmos. Chem. 27 (1997), Nr. 3, S. 291-318

[11] Šimpraga, M.; Verbeeck, H.; Demarcke, M.; Joó, É.; Pokorska, O.; Amelynck, C.; Schoon, N.; Dewulf, J.; Langenhove, H. V.; Heinesch, B.; Aubinet, M.; Laffineur, Q.; Müller, J.-F.; Steppe, K.: Clear link between drought stress, photosynthesis and biogenic volatile organic compounds in Fagus sylvatica L. In: Atmos. Environ. 45 (2011), Nr. 30, S. 5254-5259

[12] Šimpraga, M.; Verbeeck, H.; Demarcke, M.; Joó, É.; Amelynck, C.; Schoon, N.; Dewulf, J.; Langenhove, H. V.; Heinesch, B.; Aubinet, M.; Müller, J.-F.; Steppe, K.: Comparing monoterpenoid emissions and net photosynthesis of beech (Fagus sylvatica L.) in controlled and natural conditions. In: Atmos. Environ. 45 (2011), Nr. 17, S. 2922-2928

[13] Tollsten, L.; Müller, P. M.: Volatile organic compounds emitted from beech leaves. In: Phytochemistry 43 (1996), Nr. 4, S. 759-762 
A.2. Stamm-VOC von F. sylvatica 


\begin{tabular}{|c|c|c|c|c|c|c|c|c|c|c|c|c|c|c|c|c|c|c|c|}
\hline \multirow{2}{*}{ LRI Lit } & \multirow{2}{*}{ LRI } & \multirow{2}{*}{ RT } & \multirow{2}{*}{ VOC: Unterlüß } & \multirow{2}{*}{ CAS } & \multicolumn{2}{|c|}{ Mai } & \multicolumn{2}{|c|}{ Juni } & \multicolumn{2}{|c|}{ Juli } & \multicolumn{2}{|c|}{ August } & \multicolumn{2}{|c|}{ September } & \multicolumn{2}{|c|}{ November } & \multicolumn{2}{|c|}{ Dezember } & \multirow{2}{*}{ Stoffklasse } \\
\hline & & & & & $S$ & $\mathrm{~L}$ & $s$ & $\mathrm{~L}$ & s & $\mathrm{L}$ & \begin{tabular}{|l|}
$s$ \\
\end{tabular} & $\mathrm{~L}$ & s & $\mathrm{L}$ & \begin{tabular}{|l|}
$s$ \\
\end{tabular} & L & s & $\mathrm{TL}$ & \\
\hline 742 & $<800$ & 6,048 & 2-Methylpentanal & 000123-15-9 & 0 & 0 & 0 & 5 & 0 & 2 & 0 & 0 & 0 & 3 & 0 & 0 & 0 & 0 & Aldehyd \\
\hline 806 & $<800$ & 6,471 & Toluen & $000108-88-3$ & 7 & 7 & 6 & 0 & 3 & 3 & \begin{tabular}{c|c}
7 \\
\end{tabular} & 6 & $\begin{array}{lll}7 & & \\
\end{array}$ & 7 & 6 & 7 & 6 & 0 & Aromat \\
\hline 737 & $<800$ & 6,893 & 2-Ethyl-4-methyl-1-penten & $003404-80-6$ & 0 & 0 & 0 & 0 & 0 & 0 & 0 & 0 & 0 & 0 & 3 & 0 & 4 & 0 & Alken \\
\hline 766 & $<800$ & 6,932 & 2-Ethylhexen & $001632-16-2$ & 0 & 6 & 0 & 0 & 0 & 0 & 0 & 0 & 0 & 0 & 0 & 0 & 0 & 0 & Alken \\
\hline 785 & $<800$ & 6,978 & 1-Octen & 000111-66-0 & 0 & 0 & 0 & 0 & 2 & 0 & 0 & 0 & 0 & 0 & 0 & 0 & 0 & 0 & Alken \\
\hline 800 & $<800$ & 7,117 & Octan & 000111-65-9 & 5 & 6 & 0 & 5 & 5 & 4 & 4 & 0 & 5 & 3 & 4 & 4 & 4 & 0 & Alkan \\
\hline \multirow[t]{2}{*}{769} & 802 & 7,209 & Hexanal & $000066-25-1$ & 5 & 0 & 6 & 7 & 0 & 5 & 2 & 0 & 5 & 5 & 0 & 0 & 0 & 0 & Aldehyd \\
\hline & 838 & 8,053 & $?$ & & 0 & 0 & 6 & 7 & 0 & 5 & 0 & 0 & 6 & 6 & 0 & 0 & 0 & 0 & Unbekannt \\
\hline 854 & 864 & 8,667 & Ethylbenzen & 000100-41-4 & 7 & 7 & 7 & 3 & 0 & 6 & 7 & 5 & 7 & 7 & 5 & 7 & 6 & 0 & Aromat \\
\hline 862 & 872 & 8,865 & p-Xylen & $000108-38-3$ & 7 & 6 & 6 & 4 & 6 & 4 & 7 & 5 & 7 & 7 & 6 & 7 & 6 & 0 & Aromat \\
\hline 852 & 876 & 8,944 & 1-Hexanol & $000111-27-3$ & 0 & 0 & 0 & 4 & 0 & 0 & 0 & 0 & 0 & 0 & 0 & 0 & 0 & 0 & Alkohol \\
\hline 870 & 883 & 9,115 & Phenylethyn & 000536-74-3 & 0 & 0 & 0 & 0 & 0 & 0 & 7 & 6 & 0 & 0 & 0 & 0 & 0 & 0 & Aromat \\
\hline 865 & 890 & 9,293 & 3-Heptanon & 000106-35-4 & 0 & 0 & 3 & 3 & 0 & 0 & 0 & 0 & 0 & 5 & 0 & 0 & 0 & 0 & Keton \\
\hline 896 & 891 & 9,313 & 1-Nonen & $000124-11-8$ & 4 & 0 & 0 & 0 & 0 & 0 & 0 & 0 & 0 & 0 & 0 & 0 & 0 & 0 & Alken \\
\hline 907 & 896 & 9,425 & Xylen & $001330-20-7$ & 7 & 7 & 0 & 0 & 3 & 0 & 7 & 6 & 7 & 7 & 6 & 7 & 6 & 0 & Aromat \\
\hline 900 & 899 & 9,504 & Nonan & $000111-84-2$ & 5 & 3 & 5 & 5 & 4 & 2 & 0 & 0 & 4 & 0 & 0 & 0 & 0 & 0 & Alkan \\
\hline 879 & 905 & 9,630 & Heptanal & 000111-71-7 & 5 & 4 & 6 & 7 & 4 & 5 & 2 & 0 & 5 & 4 & 0 & 0 & 0 & 0 & Aldehyd \\
\hline 900 & 924 & 10,078 & Methoxybenzen & $000100-66-3$ & 0 & 6 & 0 & 0 & 0 & 2 & 0 & 0 & 0 & 0 & 0 & 3 & 0 & 0 & Ether \\
\hline 922 & 924 & 10,085 & Tricyclen & $000508-32-7$ & 0 & 0 & 0 & 0 & 0 & 0 & 0 & 0 & 0 & 0 & 4 & 0 & 0 & 0 & Monoterpen \\
\hline 928 & 930 & 10,210 & $\alpha$-Thujen & $002867-05-2$ & 0 & 0 & 0 & 0 & 4 & 6 & 0 & 0 & 0 & 0 & 0 & 0 & 0 & 0 & Monoterpen \\
\hline 930 & 937 & 10,369 & $\alpha$-Pinen & $000080-56-8$ & 5 & 7 & 6 & 7 & 5 & 7 & 5 & 2 & 7 & 7 & 4 & 3 & 4 & 0 & Monoterpen \\
\hline 922 & 945 & 10,560 & 5-Methyl-3-heptanon & $000541-85-5$ & 0 & 0 & 0 & 0 & 0 & 0 & 0 & 0 & 0 & 0 & 0 & 3 & 0 & 0 & Keton \\
\hline 943 & 952 & 10,732 & Camphen & $000079-92-5$ & 6 & 7 & 0 & 4 & 6 & 7 & 2 & 0 & 7 & 7 & 4 & 4 & 3 & 0 & Monoterpen \\
\hline 935 & 958 & 10,857 & 2-Ethylhexanal & 000123-05-7 & 0 & 0 & 4 & 7 & 3 & 4 & 0 & 0 & 5 & 5 & 4 & 7 & 0 & 0 & Aldehyd \\
\hline 952 & 966 & 11,055 & 1-Ethyl-3-methylbenzen & $000620-14-4$ & 5 & 0 & 0 & 0 & 0 & 0 & 0 & 0 & 7 & 0 & 5 & 0 & 4 & 0 & Aromat \\
\hline 972 & 970 & 11,134 & Benzaldehyd & $000100-52-7$ & 3 & 4 & 5 & 6 & 3 & 0 & 7 & 5 & 4 & 2 & 0 & 7 & 0 & 0 & Monoterpen \\
\hline 964 & 977 & 11,305 & Sabinen & $003387-41-5$ & 7 & 7 & 6 & 7 & 6 & 7 & 0 & 0 & 0 & 0 & 0 & 0 & 0 & 0 & Monoterpen \\
\hline 972 & 981 & 11,384 & $\beta$-Pinen & $000127-91-3$ & 0 & 7 & 0 & 2 & 0 & 0 & 5 & 0 & 7 & 7 & 3 & 2 & 3 & 0 & Monoterpen \\
\hline \multirow[t]{2}{*}{988} & 984 & 11,463 & 1-Ethyl-2-methylbenzen & 000611-14-3 & 0 & 0 & 0 & 0 & 0 & 0 & 0 & 0 & 0 & 0 & 0 & 7 & 0 & 0 & Aromat \\
\hline & 986 & 11,510 & ? & & 0 & 0 & 0 & 0 & 0 & 0 & 0 & 0 & 0 & 0 & 4 & 0 & 2 & 0 & Unbekannt \\
\hline 938 & 990 & 11,595 & 6-Methyl-5-hepten-2-on & $000110-93-0$ & 3 & 0 & 5 & 0 & 0 & 0 & 0 & 0 & 0 & 0 & 0 & 0 & 0 & 0 & Keton \\
\hline 963 & 991 & 11,635 & 1-Hexyl-2-methylcyclopropan & $062238-09-9$ & 0 & 0 & 0 & 0 & 0 & 0 & 0 & 0 & 0 & 0 & 5 & 0 & 0 & 0 & Alkan \\
\hline 996 & 992 & 11,641 & $2,2,4,6,6$-Pentamethylheptan & $013475-82-6$ & 5 & 6 & 0 & 5 & 0 & 0 & 0 & 6 & 7 & 6 & 0 & 7 & 3 & 0 & Alkan \\
\hline 1005 & 997 & 11,767 & 1,2,3-Trimethylbenzen & $000526-73-8$ & 0 & 3 & 0 & 0 & 0 & 0 & 0 & 0 & 0 & 0 & 0 & 5 & 5 & 0 & Aromat \\
\hline
\end{tabular}




\begin{tabular}{|c|c|c|c|c|c|c|c|c|c|c|c|c|c|c|c|c|c|c|c|}
\hline \multirow{2}{*}{ LRI Lit } & \multirow{2}{*}{ LRI } & \multirow{2}{*}{ RT } & \multirow{2}{*}{ VOC: Unterlüß } & \multirow{2}{*}{ CAS } & \multicolumn{2}{|c|}{ Mai } & \multicolumn{2}{|c|}{ Juni } & \multicolumn{2}{|c|}{ Juli } & \multicolumn{2}{|c|}{ August } & \multicolumn{2}{|c|}{\begin{tabular}{|l|} 
September \\
\end{tabular}} & \multicolumn{2}{|c|}{\begin{tabular}{|l|} 
November \\
\end{tabular}} & \multicolumn{2}{|c|}{\begin{tabular}{|l|} 
Dezember \\
\end{tabular}} & \multirow{2}{*}{ Stoffklasse } \\
\hline & & & & & $\mathbf{s}$ & $\mathrm{L}$ & $s$ & $\mathrm{~L}$ & $s$ & $\mathrm{~L}$ & $\mathbf{s}$ & $\mathrm{L}$ & \begin{tabular}{|l|l|l}
$S$ & $L$ \\
\end{tabular} & \begin{tabular}{|l|l}
$L$ &
\end{tabular} & \begin{tabular}{|l|l|}
$S$ & $L$ \\
\end{tabular} & \begin{tabular}{|l|l}
$\mathrm{L}$ &
\end{tabular} & \begin{tabular}{|l|l|} 
S & L \\
\end{tabular} & $\mathrm{L}$ & \\
\hline 1000 & 1000 & 11,826 & Decan & 000124-18-5 & 0 & 0 & 4 & 0 & 0 & 0 & 0 & 0 & 0 & 0 & 0 & 0 & 0 & 0 & Alkan \\
\hline 981 & 1005 & 11,951 & Octanal & 000124-13-0 & 5 & 0 & 5 & 6 & 0 & 2 & 7 & 0 & 6 & 4 & 0 & 1 & 0 & 0 & Aldehyd \\
\hline 1007 & 1008 & 12,011 & I-Phellandren & 000099-83-2 & 0 & 0 & 0 & 0 & 2 & 4 & 0 & 0 & 0 & 0 & 0 & 0 & 0 & 0 & Monoterpen \\
\hline 1005 & 1014 & 12,136 & 3-Caren & 013466-78-9 & 7 & 7 & 6 & 7 & 6 & 7 & 4 & 2 & 7 & 7 & 4 & 6 & 4 & 0 & Monoterpen \\
\hline 1017 & 1020 & 12,275 & $\alpha$-Terpinen & $000099-86-5$ & 0 & 0 & 0 & 7 & 5 & 7 & 0 & 0 & 0 & 0 & 0 & 0 & 0 & 0 & Monoterpen \\
\hline 1011 & 1029 & 12,459 & p-Cymol & 000099-87-6 & 6 & 7 & 0 & 6 & 5 & 7 & 0 & 0 & 0 & 4 & 5 & 6 & 0 & 0 & Aromat \\
\hline 1019 & 1033 & 12,545 & Limonen & 000138-86-3 & 7 & 7 & 6 & 7 & 6 & 7 & 5 & 4 & 5 & 7 & 3 & 6 & 4 & 0 & Monoterpen \\
\hline 1030 & 1034 & 12,565 & $\beta$-Phellandren & $000555-10-2$ & 0 & 0 & 0 & 0 & 0 & 6 & 0 & 0 & 0 & 0 & 0 & 0 & 0 & 0 & Monoterpen \\
\hline 1023 & 1036 & 12,624 & Eukalyptol & $000470-82-6$ & 6 & 6 & 6 & 6 & 6 & 7 & 2 & 0 & 3 & 2 & 2 & 0 & 0 & 0 & Monoterpen \\
\hline 1013 & 1040 & 12,710 & 2,6,6-Trimethylcyclohexanon & 002408-37-9 & 0 & 0 & 0 & 0 & 0 & 0 & 0 & 0 & 3 & 0 & 0 & 0 & 0 & 0 & Keton \\
\hline \multirow[t]{3}{*}{1031} & 1045 & 12,809 & Methyl 2-ethylhexanoat & 000816-19-3 & 7 & 5 & 0 & 0 & 6 & 3 & 0 & 0 & 4 & 3 & 2 & 0 & 0 & 0 & Ester \\
\hline & 1054 & 13,013 & ? & & 0 & 0 & 0 & 0 & 0 & 0 & 6 & 5 & 0 & 0 & 0 & 7 & 0 & 0 & Unbekannt \\
\hline & 1059 & 13,106 & ? & & 0 & 0 & 7 & 0 & 0 & 6 & 0 & 0 & 5 & 7 & 5 & 0 & 3 & 0 & Unbekannt \\
\hline 1053 & 1063 & 13,211 & $\mathrm{Y}$-Terpinen & $000099-85-4$ & 0 & 5 & 0 & 7 & 5 & 7 & 0 & 0 & 0 & 0 & 0 & 3 & 0 & 0 & Monoterpen \\
\hline \multirow[t]{2}{*}{1052} & 1078 & 13,534 & Acetophenon & 000098-86-2 & 0 & 0 & 4 & 7 & 3 & 0 & 7 & 6 & 0 & 0 & 0 & 4 & 0 & 0 & Keton \\
\hline & 1090 & 13,792 & $?$ & & 0 & 0 & 0 & 0 & 0 & 0 & 0 & 0 & 0 & 0 & 0 & 3 & 0 & 0 & \\
\hline 1080 & 1093 & 13,851 & $\alpha$-Terpinolen & 000586-62-9 & 0 & 2 & 0 & 4 & 2 & 7 & 0 & 0 & 0 & 0 & 0 & 0 & 0 & 0 & Monoterpen \\
\hline \multirow[t]{2}{*}{1100} & 1099 & 13,983 & Undecan & 001120-21-4 & 4 & 0 & 4 & 0 & 0 & 4 & 0 & 0 & 0 & 0 & 0 & 4 & 2 & 0 & Alkan \\
\hline & 1101 & 14,036 & $?$ & & 0 & 2 & 0 & 0 & 0 & 6 & 0 & 0 & 0 & 0 & 0 & 5 & 0 & 0 & Unbekannt \\
\hline \multirow[t]{3}{*}{1128} & 1107 & 14,141 & Nonanal & 000124-19-6 & 7 & 5 & 6 & 7 & 7 & 7 & 7 & 7 & 5 & 5 & 1 & 5 & 2 & 0 & Aldehyd \\
\hline & 1137 & 14,768 & $?$ & & 0 & 0 & 0 & 0 & 0 & 4 & 0 & 0 & 0 & 0 & 0 & 0 & 0 & 0 & Unbekannt \\
\hline & 1143 & 14,873 & ? & & 7 & 0 & 6 & 5 & 6 & 6 & 3 & 0 & 0 & 0 & 0 & 2 & 0 & 0 & Unbekannt \\
\hline 1146 & 1145 & 14,919 & 5-Ethyldecan & 017302-36-2 & 0 & 0 & 0 & 0 & 0 & 3 & 0 & 0 & 0 & 0 & 0 & 0 & 0 & 0 & Alkan \\
\hline \multirow[t]{2}{*}{1142} & 1148 & 14,979 & $(1 \mathrm{R})-(+)$-Norinone & 038651-65-9 & 7 & 0 & 6 & 7 & 3 & 3 & 0 & 0 & 0 & 0 & 0 & 0 & 0 & 0 & Keton \\
\hline & 1151 & 15,051 & ? & & 0 & 7 & 7 & 7 & 6 & 7 & 7 & 6 & 0 & 7 & 6 & 7 & 6 & 0 & Unbekannt \\
\hline 1121 & 1154 & 15,111 & Campher & $000076-22-2$ & 6 & 7 & 6 & 7 & 7 & 6 & 0 & 0 & 0 & 0 & 0 & 0 & 0 & 0 & Monoterpen \\
\hline 1126 & 1168 & 15,381 & Sabina ketone & 000513-20-2 & 0 & 0 & 6 & 0 & 0 & 3 & 0 & 0 & 0 & 0 & 0 & 0 & 0 & 0 & Keton \\
\hline 1143 & 1185 & 15,724 & 3-Pinanon & 000547-60-4 & 6 & 5 & 0 & 0 & 0 & 4 & 0 & 0 & 0 & 0 & 0 & 0 & 0 & 0 & Keton \\
\hline 1160 & 1196 & 15,948 & Benzoic acid & 000065-85-0 & 0 & 0 & 0 & 0 & 0 & 0 & 6 & 5 & 0 & 0 & 0 & 0 & 0 & 0 & organ. Säure \\
\hline 1200 & 1199 & 16,014 & Dodecan & 000112-40-3 & 5 & 0 & 5 & 7 & 0 & 4 & 4 & 4 & 0 & 0 & 5 & 7 & 2 & 0 & Alkan \\
\hline \multirow[t]{2}{*}{1183} & 1208 & 16,186 & Decanal & 000112-31-2 & 6 & 0 & 7 & 5 & 3 & 6 & 7 & 6 & 0 & 0 & 0 & 5 & 0 & 0 & Aldehyd \\
\hline & 1213 & 16,285 & ? & & 0 & 0 & 0 & 0 & 4 & 0 & 0 & 0 & 0 & 0 & 4 & 0 & 0 & 0 & Unbekannt \\
\hline \multirow[t]{2}{*}{1210} & 1223 & 16,476 & 5-Propyldecan & 017312-62-8 & 0 & 0 & \begin{tabular}{l|l}
0 \\
\end{tabular} & 0 & 0 & 4 & 0 & 0 & 0 & 0 & 0 & 0 & \begin{tabular}{l|l}
0 \\
\end{tabular} & 0 & Alkan \\
\hline & 1247 & 16,918 & & & 0 & 0 & 0 & 0 & 0 & 0 & 5 & 0 & 0 & 0 & 0 & 0 & 0 & 0 & Unbekannt \\
\hline
\end{tabular}




\begin{tabular}{|c|c|c|c|c|c|c|c|c|c|c|c|c|c|c|c|c|c|c|c|}
\hline \multirow{2}{*}{ LRI Lit } & \multirow{2}{*}{ LRI } & \multirow{2}{*}{ RT } & \multirow{2}{*}{ VOC: Unterlüß } & \multirow{2}{*}{ CAS } & \multicolumn{2}{|c|}{ Mai } & \multicolumn{2}{|c|}{ Juni } & \multicolumn{2}{|c|}{ Juli } & \multicolumn{2}{|c|}{ August } & \multicolumn{2}{|c|}{\begin{tabular}{|l|} 
September \\
\end{tabular}} & \multicolumn{2}{|c|}{ November } & \multicolumn{2}{|c|}{ Dezember } & \multirow{2}{*}{ Stoffklasse } \\
\hline & & & & & $S$ & L & $s$ & $\mathrm{~L}$ & $S$ & L & \begin{tabular}{|l|l|}
$s$ \\
\end{tabular} & $\mathrm{~L}$ & s & \begin{tabular}{|l|l}
$\mathrm{L}$ & \\
\end{tabular} & \begin{tabular}{|l|l}
$S$ & $L$ \\
\end{tabular} & $\mathrm{~L}$ & \begin{tabular}{|l|l}
$\mathbf{S}$ & $\mathrm{L}$ \\
\end{tabular} & $\mathrm{L}$ & \\
\hline \multirow{2}{*}{1255} & 1275 & 17,452 & 1-Decanol & 000112-30-1 & 0 & 4 & 0 & 0 & 0 & 0 & 7 & 6 & 0 & 0 & 0 & 2 & 0 & 0 & Alkohol \\
\hline & 1280 & 17,551 & ? & & 0 & 0 & 0 & 7 & 0 & 4 & 0 & 0 & 0 & 3 & 0 & 3 & 0 & 0 & Unbekannt \\
\hline 1277 & 1294 & 17,815 & Bornylacetat & $000076-49-3$ & 6 & 0 & 0 & 5 & 0 & 0 & 0 & 0 & 0 & 0 & 0 & 0 & 0 & 0 & Acetat \\
\hline \multirow{2}{*}{1300} & 1299 & 17,907 & Tridecan & 000629-50-5 & 6 & 0 & 7 & 0 & 0 & 0 & 6 & 6 & 0 & 0 & 2 & 7 & 5 & 0 & Alkan \\
\hline & 1311 & 18,125 & & & 0 & 0 & 0 & 0 & 5 & 0 & 7 & 4 & 0 & 0 & 0 & 0 & 0 & 0 & Unbekannt \\
\hline \multirow{8}{*}{1329} & 1328 & 18,429 & $2,2,4,4,6,8,8$-Heptamethylnonan & 004390-04-9 & 3 & 0 & 0 & 0 & 0 & 0 & 0 & 0 & 0 & 2 & 5 & 6 & 0 & 0 & Alkan \\
\hline & 1331 & 18,481 & ? & & 0 & 0 & 0 & 0 & 0 & 0 & 7 & 0 & 0 & 0 & 0 & 0 & 0 & 0 & Unbekannt \\
\hline & 1335 & 18,554 & $?$ & & 0 & 0 & 0 & 0 & 0 & 3 & 0 & 5 & 0 & 0 & 0 & 0 & 0 & 0 & Unbekannt \\
\hline & 1335 & 18,560 & $?$ & & 0 & 0 & 0 & 0 & 3 & 0 & 0 & 0 & 0 & 0 & 0 & 0 & 0 & 0 & Unbekannt \\
\hline & 1350 & 18,824 & $?$ & & 0 & 0 & 0 & 0 & 0 & 0 & 0 & 5 & 0 & 0 & 0 & 0 & 0 & 0 & Unbekannt \\
\hline & 1362 & 19,035 & $?$ & & 6 & 0 & 6 & 0 & 6 & 0 & 5 & 3 & 0 & 0 & 0 & 0 & 0 & 0 & Unbekannt \\
\hline & 1366 & 19,101 & & & 0 & 0 & 0 & 0 & 0 & 7 & 0 & 4 & 0 & 0 & 0 & 0 & 0 & & Unbekannt \\
\hline & 1379 & 19,338 & $?$ & & 0 & 0 & 0 & 0 & 0 & 0 & 5 & 0 & 0 & 0 & 0 & 0 & 0 & 0 & Unbekannt \\
\hline 1376 & 1388 & 19,497 & $\alpha$-Copaen & $003856-25-5$ & 7 & 0 & 0 & 0 & 0 & 0 & 0 & 0 & 0 & 0 & 2 & 0 & 0 & 0 & Sesquiterpen \\
\hline \multirow{2}{*}{1376} & 1388 & 19,503 & Longicyclen & $001137-12-8$ & 0 & 6 & 0 & 4 & 0 & 3 & 0 & 3 & 0 & 0 & 0 & 3 & 0 & & Sesquiterpen \\
\hline & 1393 & 19,583 & & & 0 & 0 & 5 & 0 & 6 & 0 & 7 & 5 & 0 & 0 & 0 & 3 & 0 & & Unbekannt \\
\hline 1403 & 1395 & 19,622 & $\alpha$-Patchoulen & 000560-32-7 & 0 & 4 & 0 & 0 & 0 & 5 & 0 & 0 & 0 & 0 & 0 & 0 & 0 & & Sesquiterpen \\
\hline \multirow{2}{*}{1400} & 1399 & 19,688 & Tetradecan & 000629-59-4 & 6 & 0 & 6 & 0 & 0 & 0 & 6 & 6 & 0 & 0 & 2 & 7 & 0 & 0 & Alkan \\
\hline & 1411 & \begin{tabular}{|l|}
19,899 \\
\end{tabular} & & & 0 & 0 & 0 & 0 & 0 & 0 & 0 & 5 & 0 & 0 & 0 & 0 & 0 & & Unbekannt \\
\hline \multirow[t]{2}{*}{1461} & 1413 & 19,919 & Y-Gurjunen & 022567-17-5 & 6 & 5 & 0 & 0 & 0 & 0 & 0 & 0 & 0 & 0 & 0 & 0 & 0 & 0 & Sesquiterpen \\
\hline & 1416 & \begin{tabular}{|l|}
19,972 \\
\end{tabular} & Tetraethylbenzen & 033637-20-6 & 5 & 7 & 0 & 4 & 0 & 0 & 0 & 0 & 0 & 0 & 0 & 0 & 0 & 0 & Aromat \\
\hline 1417 & 1425 & 20,123 & Junipen & 000475-20-7 & 7 & 7 & 5 & 7 & 7 & 6 & 7 & 6 & 6 & 6 & 4 & 6 & 4 & 0 & Sesquiterpen \\
\hline \multirow[t]{3}{*}{1436} & 1451 & 20,559 & $\alpha$-Gurjunen & 000489-40-7 & 0 & 3 & 0 & 0 & 0 & 2 & 5 & 4 & 5 & 2 & 3 & 5 & 0 & 0 & Sesquiterpen \\
\hline & 1456 & 20,651 & & 999192-20-2 & 0 & 5 & 5 & 5 & 6 & 6 & 2 & 0 & 0 & 3 & 0 & 0 & 0 & 0 & Unbekannt \\
\hline & 1468 & 20,855 & $?$ & & 0 & 0 & 0 & 0 & 0 & 4 & 0 & 5 & 0 & 0 & 0 & 0 & 0 & 0 & Unbekannt \\
\hline 1419 & 1469 & 20,869 & (-)-Isoleden & 095910-36-4 & 0 & 0 & 0 & 0 & 7 & 3 & 5 & 0 & 0 & 0 & 0 & 0 & \begin{tabular}{l|l}
0 & \\
\end{tabular} & 0 & Sesquiterpen \\
\hline 1499 & 1497 & 21,330 & trans- $\beta$-Farnesen & 000502-60-3 & 0 & 0 & 0 & 0 & 0 & 0 & 0 & 0 & 0 & 0 & 4 & 3 & 0 & 0 & Sesquiterpen \\
\hline 1500 & 1498 & 21,357 & Pentadecan & 000629-62-9 & 6 & 0 & 4 & 0 & 0 & 0 & 0 & 3 & 3 & 5 & 2 & 4 & 3 & 0 & Alkan \\
\hline 1491 & 1499 & 21,363 & Cadinen & 999191-97-0 & 0 & 4 & 0 & 0 & 7 & 6 & 7 & 0 & 0 & 0 & 0 & 0 & 0 & 0 & Sesquiterpen \\
\hline 1483 & 1505 & 21,456 & $\beta$-Selinen & 017066-67-0 & 0 & 3 & 0 & 0 & 0 & 6 & 0 & 0 & 0 & 0 & 0 & 0 & 0 & 0 & Sesquiterpen \\
\hline \multirow[t]{2}{*}{1500} & 1505 & 21,462 & $\alpha$-Selinen & 000473-13-2 & 0 & 0 & 0 & 0 & 7 & 0 & 3 & 0 & 0 & 0 & 0 & 0 & 0 & 0 & Sesquiterpen \\
\hline & 1513 & 21,588 & ? & & 0 & 0 & 0 & 0 & 0 & 6 & 0 & 2 & 0 & 0 & 0 & 0 & 0 & 0 & Unbekannt \\
\hline 1494 & 1513 & 21,594 & $\alpha$-Muurolen & 031983-22-9 & 0 & 0 & 0 & 0 & 5 & 0 & 7 & 0 & 0 & 0 & 0 & 0 & 0 & 0 & Sesquiterpen \\
\hline 1507 & 1520 & 21,701 & $\psi$-Cadinen & 039029-41-9 & 0 & 0 & 0 & 0 & 5 & 0 & 0 & 0 & 0 & 0 & 0 & 0 & 0 & & Sesquiterpen \\
\hline
\end{tabular}




\begin{tabular}{|c|c|c|c|c|c|c|c|c|c|c|c|c|c|c|c|c|c|c|c|}
\hline \multirow{2}{*}{ LRI Lit } & \multirow{2}{*}{ LRI } & \multirow{2}{*}{ RT } & \multirow{2}{*}{ VOC: Unterlüß } & \multirow{2}{*}{ CAS } & \multicolumn{2}{|c|}{ Mai } & \multicolumn{2}{|c|}{ Juni } & \multicolumn{2}{|c|}{ Juli } & \multicolumn{2}{|c|}{ August } & \multicolumn{2}{|c|}{ September } & \multicolumn{2}{|c|}{ November } & \multicolumn{2}{|c|}{ Dezember } & \multirow{2}{*}{ Stoffklasse } \\
\hline & & & & & $S$ & $\mathrm{~L}$ & S & $\bar{L}$ & $S$ & $\mathrm{TL}$ & \begin{tabular}{|l|l|}
$s$ \\
\end{tabular} & $\mathrm{~L}$ & \begin{tabular}{|l|l|}
$\mathbf{S}$ & $\mathrm{a}$ \\
\end{tabular} & $\mathrm{L}$ & \begin{tabular}{|l|l}
$S$ & $L$ \\
\end{tabular} & \begin{tabular}{|l|l}
$\mathrm{L}$ \\
\end{tabular} & \begin{tabular}{|l|l}
$\mathbf{S}$ & $\mathrm{t}$ \\
\end{tabular} & $\mathrm{L}$ & \\
\hline & 1542 & 22,056 & ? & & 0 & 0 & 0 & 0 & 0 & 0 & 0 & 6 & 0 & 2 & 0 & 0 & 0 & 0 & Unbekannt \\
\hline & 1544 & 22,089 & ? & & 0 & 0 & 0 & 0 & 0 & 0 & 6 & 0 & 0 & 0 & 0 & 0 & 0 & 0 & Unbekannt \\
\hline & 1550 & 22,181 & $?$ & & 0 & 0 & 0 & 0 & 0 & 0 & 0 & 0 & 0 & 0 & 0 & 4 & 0 & 0 & Unbekannt \\
\hline \multirow[t]{2}{*}{1551} & 1556 & 22,280 & n-Nonylcyclohexan & 002883-02-5 & 0 & 0 & 0 & 0 & 0 & 0 & 0 & 0 & 0 & 0 & 0 & 3 & 0 & 0 & Alkan \\
\hline & 1558 & 22,313 & ? & & 0 & 0 & 0 & 0 & 0 & 0 & 0 & 4 & 0 & 0 & 0 & 0 & 0 & 0 & Unbekannt \\
\hline 1568 & 1570 & 22,498 & 3-Methylpentadecan & 002882-96-4 & 0 & 0 & 0 & 0 & 6 & 0 & 0 & 0 & 0 & 0 & 3 & 2 & 0 & 0 & Alkan \\
\hline 1600 & 1598 & 22,946 & Hexadecan & 000544-76-3 & 3 & 0 & 0 & 0 & 5 & 0 & 0 & 5 & 4 & 5 & 3 & 6 & 0 & 0 & Alkan \\
\hline 1578 & 1606 & 23,059 & Diethylphthalat & $000084-66-2$ & 4 & 0 & 0 & 0 & 0 & 0 & 6 & 0 & 0 & 0 & 0 & 0 & 0 & 0 & Ester \\
\hline \multirow[t]{2}{*}{1611} & 1652 & 23,744 & Benzophenon & 000119-61-9 & 5 & 0 & 0 & 0 & 0 & 0 & 2 & 2 & 0 & 0 & 0 & 0 & 0 & 0 & Keton \\
\hline & 1672 & 24,054 & 6(E),8(E)-Heptadecadien & 999281-78-6 & 0 & 0 & 6 & 5 & 0 & 3 & 0 & 0 & 0 & 0 & 2 & 4 & 0 & 0 & Alken \\
\hline \multirow[t]{2}{*}{1719} & 1680 & 24,166 & 8-Heptadecen & 002579-04-6 & 6 & 2 & 6 & 6 & 0 & 5 & 3 & 0 & 0 & 3 & 2 & 3 & 0 & 0 & Alken \\
\hline & 1685 & 24,252 & ? & & 0 & 0 & 0 & 0 & 0 & 0 & 7 & 6 & 0 & 0 & 0 & 2 & 0 & 0 & Unbekannt \\
\hline 1700 & 1698 & 24,444 & Heptadecan & 000629-78-7 & 0 & 0 & 0 & 0 & 0 & 0 & 0 & 0 & 0 & 2 & \begin{tabular}{l|l}
0 \\
\end{tabular} & 3 & 0 & 0 & Alkan \\
\hline
\end{tabular}

LRI Lit = linearer Retentionsindex in der Literatur; LRI =linearer Retetionsindex; VOC = flüchtige organische Verbindung; CAS = Chemical Abstracts Service Number; $S=$ Sand; $L=L$ Lehm; $?=$ nicht identifiziertes VOC 


\begin{tabular}{|c|c|c|c|c|c|c|c|c|c|c|c|c|c|c|c|c|c|c|c|}
\hline \multirow{2}{*}{ LRI Lit } & \multirow{2}{*}{ LRI } & \multirow{2}{*}{ RT } & \multirow{2}{*}{ VOC: Göhrde } & \multirow{2}{*}{ CAS } & \multicolumn{2}{|c|}{ Mai } & \multicolumn{2}{|c|}{ Juni } & \multicolumn{2}{|c|}{ Juli } & \multicolumn{2}{|c|}{ August } & \multicolumn{2}{|c|}{\begin{tabular}{|l|} 
September \\
\end{tabular}} & \multicolumn{2}{|c|}{ Oktober } & \multicolumn{2}{|c|}{\begin{tabular}{|l|} 
Dezember \\
\end{tabular}} & \multirow{2}{*}{ Stoffklasse } \\
\hline & & & & & $S$ & $\mathrm{~L}$ & 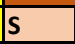 & $\mathrm{L}$ & $\mathrm{s}$ & L & $\mathrm{s}$ & $\overline{\mathrm{L}}$ & \begin{tabular}{|l|l|}
$s$ \\
\end{tabular} & 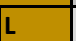 & \begin{tabular}{|l|l|l|}
$\mathbf{s}$ & $\mathrm{a}$
\end{tabular} & L & \begin{tabular}{|l|l|l}
$s$ & 1 \\
\end{tabular} & $\mathrm{~L}$ & \\
\hline 707 & $<800$ & 5,059 & Pentanal & $000110-62-3$ & 2 & 6 & 4 & 4 & 4 & 0 & 3 & 0 & 2 & 0 & 0 & 0 & 0 & 0 & Aldehyd \\
\hline 736 & $<800$ & 6,015 & 2-Methylpentanal & $000123-15-9$ & 0 & 7 & 5 & 6 & 6 & 7 & 7 & 7 & 0 & 5 & 5 & 0 & 0 & 0 & Aldehyd \\
\hline 716 & $<800$ & 6,141 & 2-Isopropyl-2-methyloxiran & $072221-03-5$ & 0 & 0 & & 0 & 0 & 0 & 4 & 0 & 0 & 0 & 0 & 0 & 0 & 0 & Ether \\
\hline 806 & $<800$ & 6,457 & Toluen & $000108-88-3$ & 5 & 4 & & 5 & 7 & 6 & 7 & 7 & 7 & 7 & 7 & 7 & 7 & 7 & Aromat \\
\hline 785 & $<800$ & 6,899 & 1-Octen & 000111-66-0 & 0 & 6 & & 4 & 5 & 6 & 5 & 6 & 3 & 4 & 0 & 0 & 4 & 7 & Alken \\
\hline 800 & $<800$ & 7,077 & Octan & $000111-65-9$ & 0 & 4 & & 2 & 6 & 4 & 3 & 6 & 7 & 6 & 7 & 4 & 6 & 7 & $\begin{array}{l}\text { Alkan } \\
\end{array}$ \\
\hline 785 & \begin{tabular}{|l|}
803 \\
\end{tabular} & 7,229 & Hexanal & \begin{tabular}{|l|}
$000066-25-1$ \\
\end{tabular} & 7 & 7 & & 6 & 7 & 7 & 7 & 6 & 7 & 6 & 7 & 2 & 0 & 0 & Aldehyd \\
\hline 823 & 818 & 7,592 & 2,4-Dimethylheptan & 002213-23-2 & 0 & 0 & & 0 & 0 & 0 & 0 & 2 & 0 & 0 & 0 & 0 & 0 & 0 & Alkan \\
\hline & 838 & 8,060 & ? & & 5 & 6 & 6 & 6 & 7 & 7 & 7 & 6 & 5 & 6 & 7 & 0 & 0 & 0 & Unbekannt \\
\hline 854 & 864 & 8,673 & Ethylbenzen & 000100-41-4 & 0 & 3 & 5 & 4 & 7 & 7 & 5 & 7 & 7 & 7 & 7 & 6 & 7 & 7 & $\begin{array}{l}\text { Aromat } \\
\end{array}$ \\
\hline 862 & 872 & 8,865 & p-Xylen & 000108-38-3 & 3 & 7 & 0 & 5 & 7 & 7 & 7 & 4 & 7 & 7 & 7 & 7 & 6 & & Aromat \\
\hline 870 & 882 & 9,102 & Phenylethyn & 000536-74-3 & 0 & 0 & 0 & 0 & 0 & 0 & 0 & 0 & 0 & 0 & 0 & 4 & 0 & 0 & Aromat \\
\hline 865 & 890 & 9,280 & 3-Heptanon & $000106-35-4$ & 0 & 0 & 0 & 2 & 6 & 4 & 5 & 2 & 6 & 4 & 2 & 0 & 0 & 0 & Keton \\
\hline 907 & 895 & 9,412 & Xylen & $001330-20-7$ & 0 & 0 & 3 & 4 & 6 & 7 & 7 & 7 & 7 & 7 & 7 & 7 & 7 & 4 & Aromat \\
\hline 900 & 899 & 9,498 & Nonan & $000111-84-2$ & 7 & 7 & 5 & 5 & 7 & 4 & 5 & 2 & 4 & 0 & 0 & 1 & 0 & 5 & Alkan \\
\hline 879 & 904 & 9,610 & Heptanal & 000111-71-7 & 7 & 7 & 6 & 6 & 7 & 6 & 7 & 6 & 7 & 6 & 6 & 3 & 0 & 0 & Aldehyd \\
\hline 922 & 924 & 10,078 & Tricyclen & 000508-32-7 & 0 & 0 & 0 & 0 & 0 & 0 & 0 & 4 & 0 & 0 & 5 & 5 & 4 & & Monoterpen \\
\hline 928 & 930 & 10,203 & $\alpha$-Thujen & 002867-05-2 & 0 & 0 & 5 & 0 & 0 & 0 & 0 & 0 & 0 & 0 & 0 & 0 & 0 & 0 & Monoterpen \\
\hline 930 & 937 & 10,368 & $\alpha$-Pinen & 000080-56-8 & 7 & 7 & 6 & 6 & 7 & 7 & 6 & 6 & 7 & 7 & 7 & 7 & 7 & & Monoterpen \\
\hline 922 & 946 & 10,573 & 5-Methyl-3-heptanon & $000541-85-5$ & 0 & 0 & 0 & 0 & 0 & 0 & 0 & 0 & 0 & 0 & 7 & 0 & 0 & 0 & Keton \\
\hline 943 & 952 & 10,725 & Camphen & $000079-92-5$ & 3 & 7 & 5 & 4 & 2 & 7 & 0 & 4 & 0 & 7 & 7 & 7 & 6 & 6 & Monoterpen \\
\hline 933 & 957 & 10,843 & 2-Ethylhexanal & $000123-05-7$ & 6 & 5 & 5 & 4 & 7 & 0 & 7 & 4 & 7 & 3 & 6 & 0 & 0 & 0 & Aldehyd \\
\hline & 962 & 10,962 & $?$ & & 0 & 0 & 0 & 3 & 0 & 0 & 0 & 3 & 0 & 0 & 0 & 0 & 0 & 0 & Unbekannt \\
\hline 952 & 966 & 11,041 & 1-Ethyl-3-methylbenzen & $000620-14-4$ & 0 & 4 & 0 & 3 & 3 & 4 & 0 & 0 & 0 & 7 & 0 & 3 & 0 & 0 & Aromat \\
\hline 972 & 970 & 11,147 & Benzaldehyd & $000100-52-7$ & 7 & 6 & 5 & 5 & 3 & 4 & 7 & 6 & 7 & 4 & 0 & 5 & 0 & 0 & Aldehyd \\
\hline 964 & 977 & 11,292 & Sabinen & $000555-10-2$ & 7 & 0 & 6 & 0 & 7 & 7 & 0 & 6 & 5 & 0 & 0 & 0 & 0 & 0 & Monoterpen \\
\hline 972 & 981 & 11,384 & $\beta$-Pinen & $018172-67-3$ & 0 & 7 & & 6 & 0 & 5 & 7 & 6 & 4 & 7 & 7 & 7 & 4 & 6 & Monoterpen \\
\hline 996 & 989 & 11,569 & 3 -Octanon & 000106-68-3 & 0 & 0 & 0 & 0 & 2 & 0 & 0 & 0 & 0 & 2 & 0 & 0 & 0 & 0 & Keton \\
\hline 996 & 991 & 11,635 & $2,2,4,6,6$-Pentamethylheptan & $013475-82-6$ & 6 & 0 & 4 & 6 & 4 & 0 & 6 & 2 & 5 & 6 & 4 & 4 & 6 & 4 & Alkan \\
\hline 978 & 998 & 11,787 & $1,2,4$-Trimethylbenzen & $000095-63-6$ & 0 & 0 & 0 & 0 & 0 & 0 & 0 & 0 & 0 & 0 & 0 & 3 & 0 & 0 & Aromat \\
\hline 1000 & 999 & 11,819 & Decan & $000124-18-5$ & 7 & 7 & 4 & 3 & 0 & 0 & 5 & 0 & 4 & 0 & 0 & 0 & 5 & 0 & Alkan \\
\hline 981 & 1005 & 11,945 & Octanal & $000124-13-0$ & 7 & 7 & 5 & 6 & 7 & 6 & 7 & 6 & 7 & 6 & 6 & 2 & 0 & 0 & Aldehyd \\
\hline 1005 & 1014 & 12,136 & 3-Caren & $013466-78-9$ & 7 & 7 & 6 & 6 & 7 & 7 & 7 & 7 & 7 & 7 & 7 & 7 & 7 & 6 & Monoterpen \\
\hline 1017 & 1020 & 12,274 & $\alpha$-Terpinen & 000099-86-5 & 0 & 0 & 6 & 0 & 4 & 5 & 0 & 0 & 0 & 0 & 0 & 0 & 0 & 0 & Monoterpen \\
\hline 1029 & 1024 & 12,361 & 2,2,6-Trimethyloctan & $062016-28-8$ & 0 & 0 & 0 & 0 & 0 & 0 & 0 & 0 & 0 & 0 & 0 & 0 & 3 & 0 & Alkan \\
\hline 1011 & 1028 & 12,453 & p-Cymol & |000099-87-6 & 7 & 6 & 6 & 4 & 7 & 7 & 5 & 6 & 6 & 6 & 7 & 0 & 3 & & Monoterpen \\
\hline
\end{tabular}




\begin{tabular}{|c|c|c|c|c|c|c|c|c|c|c|c|c|c|c|c|c|c|c|c|}
\hline \multirow{2}{*}{ LRI Lit } & \multirow{2}{*}{ LRI } & \multirow{2}{*}{ RT } & \multirow{2}{*}{ VOC: Göhrde } & \multirow{2}{*}{ CAS } & \multicolumn{2}{|c|}{ Mai } & \multicolumn{2}{|c|}{ Juni } & \multicolumn{2}{|c|}{ Juli } & \multicolumn{2}{|c|}{ August } & \multicolumn{2}{|c|}{\begin{tabular}{|l|} 
September \\
\end{tabular}} & \multicolumn{2}{|c|}{\begin{tabular}{|l|} 
Oktober \\
\end{tabular}} & \multicolumn{2}{|c|}{ Dezember } & \multirow{2}{*}{ Stoffklasse } \\
\hline & & & & & $\mathbf{S}$ & $\mathrm{L}$ & s & $\mathrm{L}$ & $\mathbf{S}$ & L & \begin{tabular}{|l|l|l|l|}
$\mathbf{s}$ & $\mathrm{l}$
\end{tabular} & $\mathrm{L}$ & \begin{tabular}{|l|l}
$\mathbf{S}$ & $\mathrm{L}$ \\
\end{tabular} & \begin{tabular}{|l|l}
$\mathrm{L}$ & $\mathrm{S}$ \\
\end{tabular} & \begin{tabular}{l|l|l|}
$\mathbf{s}$ & $\mathbf{r}$
\end{tabular} & L & \begin{tabular}{|l|l}
$S$ & 1 \\
\end{tabular} & $\mathrm{~L}$ & \\
\hline 1010 & 1032 & 12,532 & 2-Ethylhexanol & 000104-76-7 & 0 & 0 & 0 & 0 & 0 & 0 & 0 & $4 \mid$ & 0 & 5 & 0 & 0 & \begin{tabular}{l|l}
0 & $-r a r a t$ \\
\end{tabular} & 0 & Alkohol \\
\hline 1019 & 1033 & 12,551 & Limonen & 000138-86-3 & 7 & 7 & 6 & 6 & 7 & 7 & 7 & 6 & 7 & 7 & 7 & 4 & 6 & 5 & Monoterpen \\
\hline 1023 & 1036 & 12,624 & Eukalyptol & $000470-82-6$ & 7 & 6 & 6 & 6 & 6 & 7 & 5 & 7 & 0 & 4 & 0 & 1 & 0 & 0 & Monoterpen \\
\hline 1013 & 1040 & 12,697 & 2,6,6-Trimethylcyclohexanon & 002408-37-9 & 0 & 0 & 0 & 0 & 0 & 0 & 0 & 5 & 0 & 0 & 0 & 0 & 0 & 0 & Keton \\
\hline \multirow[t]{4}{*}{1031} & 1045 & 12,809 & Methyl 2-ethylhexanoat & 000816-19-3 & 0 & 0 & 0 & 0 & 0 & 0 & 0 & 0 & 0 & 3 & 0 & 0 & 0 & 0 & Ester \\
\hline & 1053 & 12,987 & $?$ & & 0 & 0 & 0 & 0 & 0 & 0 & 0 & 3 & 0 & 0 & 0 & 0 & 0 & 0 & Unbekannt \\
\hline & 1058 & 13,099 & $?$ & & 0 & 0 & 0 & 0 & 6 & 0 & 6 & 6 & 5 & 5 & 7 & 6 & 3 & 7 & Unbekannt \\
\hline & 1059 & 13,112 & $?$ & & 0 & 0 & 0 & 0 & 0 & 0 & 7 & 0 & 0 & 0 & 0 & 0 & 0 & 0 & Unbekannt \\
\hline \multirow[t]{2}{*}{1053} & 1063 & 13,198 & $\gamma$-Terpinen & 000099-85-4 & 6 & 4 & 6 & 4 & 7 & 7 & 0 & 2 & 0 & 0 & 6 & 0 & 0 & 0 & Monoterpen \\
\hline & 1064 & 13,231 & $?$ & & 0 & 0 & 0 & 0 & 0 & 0 & 7 & 2 & 0 & 0 & 3 & 0 & 3 & 7 & Unbekannt \\
\hline \multirow{2}{*}{1049} & 1076 & 13,495 & Acetophenon & $000098-86-2$ & 6 & 5 & 4 & 5 & 3 & 2 & 5 & 6 & 3 & 4 & 0 & 5 & 0 & 0 & Keton \\
\hline & 1082 & 13,613 & ? & & 0 & 0 & 0 & 0 & 7 & 0 & 7 & 0 & 5 & 0 & 3 & 0 & 0 & 0 & Unbekannt \\
\hline \multirow{2}{*}{1080} & 1093 & 13,851 & $\alpha$-Terpinolen & $000586-62-9$ & 0 & 0 & 5 & 0 & 0 & 0 & 0 & 0 & 0 & 0 & 0 & 0 & 0 & 0 & Monoterpen \\
\hline & 1097 & 13,936 & ? & & 4 & 5 & 0 & 0 & 0 & 0 & 0 & 0 & 0 & 0 & 0 & 0 & 0 & 0 & Unbekannt \\
\hline \multirow{2}{*}{1100} & 1099 & 13,989 & Undecan & $001120-21-4$ & 5 & 5 & 0 & 5 & 5 & 0 & 6 & 7 & 4 & 4 & 0 & 4 & 0 & 6 & Alkan \\
\hline & 1102 & 14,049 & ? & & 0 & 0 & 0 & 0 & 5 & 0 & 5 & 0 & 3 & 0 & 5 & 0 & 0 & 0 & Unbekannt \\
\hline \multirow{3}{*}{1128} & 1107 & \begin{tabular}{|l|}
14,141 \\
\end{tabular} & Nonanal & 000124-19-6 & 7 & 7 & 6 & 6 & 7 & 7 & 7 & 7 & 7 & 7 & 6 & 5 & 3 & 3 & Aldehyd \\
\hline & 1135 & 14,709 & $?$ & & 0 & 4 & 0 & 0 & 0 & 0 & 4 & 4 & 0 & 0 & 0 & 0 & 0 & 0 & Unbekannt \\
\hline & 1143 & 14,874 & $?$ & & 0 & 0 & 0 & 0 & 3 & 0 & 0 & 7 & 0 & 0 & 0 & 0 & 0 & 0 & Unbekannt \\
\hline \multirow{2}{*}{1142} & 1147 & 14,966 & $(1 \mathrm{R})-(+)-$ Norinon & 038651-65-9 & 4 & 6 & 0 & 5 & 0 & 5 & 7 & 7 & 0 & 3 & 0 & 0 & 0 & 0 & Keton \\
\hline & 1151 & 15,049 & ? & & 7 & 7 & 6 & 6 & 7 & 6 & 7 & 7 & 6 & 7 & 7 & 7 & 7 & 7 & Unbekannt \\
\hline 1121 & 1154 & 15,111 & Campher & $000076-22-2$ & 2 & 4 & 3 & 5 & 3 & 3 & 5 & 6 & 2 & 6 & 0 & 4 & 0 & 0 & Monoterpen \\
\hline & 1166 & 15,355 & $?$ & & 0 & 0 & 0 & 0 & 5 & 0 & 0 & 0 & 0 & 0 & 0 & 0 & 0 & 0 & Unbekannt \\
\hline 1126 & 1168 & 15,388 & Sabina keton & 000513-20-2 & 0 & 5 & 0 & 0 & 0 & 6 & 0 & 0 & 0 & 0 & 0 & 0 & 0 & 0 & Keton \\
\hline & 1197 & 15,968 & ? & & 0 & 0 & 0 & 0 & 7 & 0 & 4 & 0 & 0 & 0 & 0 & 0 & 0 & 0 & Alkan \\
\hline 1200 & 1199 & 16,014 & Dodecan & 000112-40-3 & 6 & 4 & 0 & 3 & 6 & 5 & 7 & 7 & 4 & 5 & 0 & 6 & 4 & 0 & Alkan \\
\hline & 1200 & 16,028 & & & 0 & 0 & 0 & 0 & 0 & 6 & 0 & 0 & 0 & 0 & 0 & 0 & 3 & 0 & Unbekannt \\
\hline & 1204 & \begin{tabular}{|l|}
16,114 \\
\end{tabular} & $?$ & & 0 & 0 & 0 & 0 & 0 & 7 & 0 & 5 & 0 & 0 & 0 & 0 & 0 & 0 & Unbekannt \\
\hline 1183 & 1208 & 16,186 & Decanal & $000112-31-2$ & 6 & 6 & 6 & 6 & 6 & 0 & 7 & 7 & 7 & 3 & 0 & 5 & 2 & 0 & Aldehyd \\
\hline & 1211 & 16,245 & $?$ & & 0 & 0 & 0 & 0 & 6 & 5 & 0 & 0 & 0 & 0 & 0 & 0 & 0 & 2 & Unbekannt \\
\hline & 1223 & 16,463 & ? & & 0 & 0 & 0 & 0 & 0 & 5 & 0 & 0 & 0 & 0 & 0 & 0 & 0 & 0 & Unbekannt \\
\hline 1210 & 1223 & 16,469 & 5-Propyldecan & $017312-62-8$ & 0 & 0 & 0 & 0 & 5 & 0 & 2 & 0 & 0 & 0 & 0 & 0 & 0 & 0 & Alkan \\
\hline & 1229 & 16,588 & ? & & 0 & 0 & 0 & 0 & 0 & 4 & 0 & 0 & 0 & 0 & 0 & 0 & 0 & 0 & Unbekannt \\
\hline 1196 & 1231 & 16,614 & $\beta$-Cyclocitral & $000432-25-7$ & 0 & 0 & 2 & 0 & 0 & 0 & 0 & 5 & 0 & 0 & 0 & 0 & 0 & 0 & Aldehyd \\
\hline 1224 & 1231 & 16,621 & 2-Ethylhexylpropenoat & 000103-11-7 & 0 & 0 & 0 & 0 & 0 & 0 & $2 \mid$ & 0 & 5 & 0 & 0 & 0 & 0 & 0 & Ester \\
\hline 1208 & 1237 & 16,733 & Cyclohexanone, 4-(1,1-dimethylethyl)- & 000098-53-3 & 0 & 0 & 0 & 0 & 0 & 0 & $2 \mid$ & 0 & $3 \mid$ & 0 & 0 & 0 & 0 & 0 & Keton \\
\hline
\end{tabular}




\begin{tabular}{|c|c|c|c|c|c|c|c|c|c|c|c|c|c|c|c|c|c|c|c|}
\hline \multirow{2}{*}{ LRI Lit } & \multirow{2}{*}{ LRI } & \multirow{2}{*}{ RT } & \multirow{2}{*}{ VOC: Göhrde } & \multirow{2}{*}{ CAS } & \multicolumn{2}{|c|}{ Mai } & \multicolumn{2}{|c|}{ Juni } & \multicolumn{2}{|c|}{ Juli } & \multirow{2}{*}{\multicolumn{2}{|c|}{ August }} & \multirow{2}{*}{\multicolumn{2}{|c|}{ September }} & \multicolumn{2}{|c|}{ Oktober } & \multicolumn{2}{|c|}{ Dezember } & \multirow{2}{*}{ Stoffklasse } \\
\hline & & & & & $\mathbf{S}$ & $\mathbf{L}$ & S & $\mathrm{L}$ & s & $\mathrm{L}$ & & $\mathrm{L}$ & s & & \begin{tabular}{|l|l}
$\mathbf{s}$ & $\mathrm{t}$ \\
$\mathrm{s}$
\end{tabular} & L & \begin{tabular}{|l|l}
$\mathbf{S}$ & $\mathrm{L}$ \\
\end{tabular} & $\mathrm{L}$ & \\
\hline & 1240 & 16,786 & ? & & 0 & 0 & 0 & 0 & 0 & 0 & 0 & 0 & 4 & 0 & 0 & 0 & 0 & 0 & Unbekannt \\
\hline & 1241 & 16,819 & ? & & 0 & 0 & 0 & 0 & 4 & 0 & 0 & 0 & 0 & 0 & 0 & 0 & 0 & 0 & Unbekannt \\
\hline & 1260 & 17,168 & $?$ & & 0 & 0 & 0 & 0 & 0 & 0 & 3 & 0 & 0 & 0 & 0 & 0 & 0 & 0 & Unbekannt \\
\hline 1255 & 1274 & 17,445 & 1-Decanol & 000112-30-1 & 0 & 0 & 0 & 0 & 0 & 0 & 2 & 0 & 0 & 0 & 0 & 4 & 0 & 0 & Alkohol \\
\hline & 1280 & 17,551 & $?$ & & 0 & 0 & 0 & 0 & 0 & 0 & 0 & $\overline{6}$ & 4 & 4 & 0 & 0 & 0 & 4 & Unbekannt \\
\hline & 1281 & 17,564 & 2,6,11-Trimethyldodecan & 031295-56-4 & 0 & 0 & 0 & 0 & 0 & 0 & 4 & 0 & 0 & 0 & 0 & 0 & 0 & 0 & Alkan \\
\hline 1270 & 1294 & 17,815 & Bornylacetat & $000076-49-3$ & 0 & 0 & 0 & 3 & 0 & 0 & 0 & 0 & 0 & 0 & 0 & 0 & 0 & 0 & Ester \\
\hline 1300 & 1298 & 17,901 & Tridecan & 000629-50-5 & 3 & 6 & 0 & 6 & 0 & 0 & 7 & 6 & 6 & 3 & 0 & 6 & 5 & 4 & Alkan \\
\hline & 1311 & 18,132 & ? & & 0 & 0 & 0 & 0 & 0 & 7 & 0 & 7 & 0 & 0 & 0 & 3 & 0 & 0 & Unbekannt \\
\hline & 1322 & 18,330 & ? & & 0 & 0 & 0 & 0 & 0 & 0 & 0 & 5 & 0 & 0 & 0 & 0 & 0 & 0 & Unbekannt \\
\hline 1357 & 1331 & 18,488 & 1-Undecanol & $000112-42-5$ & 0 & 0 & 0 & 0 & 0 & 0 & 0 & 0 & 0 & 0 & 0 & 4 & 0 & 0 & Alkohol \\
\hline & 1335 & 18,554 & $?$ & & 0 & 0 & 0 & 0 & 6 & 0 & 0 & 0 & 0 & 0 & 0 & 0 & 0 & 0 & Unbekannt \\
\hline & 1352 & 18,864 & $?$ & & 0 & 0 & 0 & 0 & 0 & 0 & 0 & 3 & 0 & 0 & 0 & 0 & 0 & 0 & Unbekannt \\
\hline & 1362 & 19,028 & ? & & 0 & 0 & 2 & 3 & 7 & 3 & 3 & 6 & 4 & 0 & 0 & 0 & 0 & 0 & Sesquiterpen \\
\hline & 1389 & 19,510 & $?$ & & 0 & 0 & 0 & 5 & 0 & 0 & 3 & 4 & 0 & 4 & 0 & 4 & 0 & 0 & Unbekannt \\
\hline & 1394 & 19,609 & ? & & 0 & 0 & 0 & 0 & 6 & 0 & 6 & 0 & 2 & 0 & 0 & 0 & 0 & 0 & Sesquiterpen \\
\hline 1400 & 1398 & 19,675 & Tetradecan & 000629-59-4 & 0 & 0 & 0 & 0 & 0 & 0 & 7 & 7 & 4 & 3 & 0 & 6 & 0 & 2 & Alkan \\
\hline & 1416 & 19,972 & Tetraethylbenzen & 033637-20-6 & 0 & 0 & 0 & 0 & 0 & 0 & 0 & 0 & 0 & 0 & 0 & 0 & 7 & 7 & Aromat \\
\hline 1417 & 1425 & 20,123 & Junipen & 000475-20-7 & 4 & 6 & 4 & 6 & 5 & 3 & 5 & 7 & 0 & 7 & 4 & 2 & 0 & 0 & Sesquiterpen \\
\hline & 1428 & 20,183 & ? & & 0 & 0 & 0 & 0 & 0 & 0 & 0 & 0 & 0 & 0 & 0 & 4 & 0 & 0 & Unbekannt \\
\hline & 1456 & 20,638 & ? & & 0 & 0 & 0 & 0 & 0 & 0 & 0 & 0 & 5 & 0 & 0 & 0 & 0 & 0 & Unbekannt \\
\hline & 1456 & 20,644 & $?$ & & 5 & 0 & 0 & 0 & 7 & 3 & 6 & 0 & 0 & 0 & 0 & 0 & 0 & 0 & Unbekannt \\
\hline 1439 & 1469 & 20,862 & Aromadendren & 000489-39-4 & 0 & 0 & 0 & 0 & 0 & 0 & 0 & 4 & 0 & 0 & 0 & 0 & 0 & 0 & Sesquiterpen \\
\hline 1499 & 1497 & 21,337 & trans- $\beta$-Farnesen & $000502-60-3$ & 0 & 0 & 0 & 0 & 0 & 0 & 3 & 0 & 0 & 0 & 0 & 0 & 2 & 5 & Sesquiterpen \\
\hline 1500 & 1499 & 21,363 & Pentadecan & 000629-62-9 & 5 & 0 & 6 & 0 & 4 & 0 & 3 & 5 & 3 & 3 & 6 & 4 & 0 & 0 & Alkan \\
\hline 1483 & 1504 & 21,449 & $\beta$-Selinen & \begin{tabular}{|l|}
$017066-67-0$ \\
\end{tabular} & 0 & 0 & 0 & 0 & 4 & 0 & 2 & 0 & 0 & 0 & 0 & 0 & 0 & 0 & Sesquiterpen \\
\hline 1523 & 1512 & 21,581 & $\alpha$-Selinen & 000473-13-2 & 0 & 0 & 0 & 0 & 5 & 6 & 3 & 6 & 0 & 0 & 0 & 0 & 0 & 0 & Sesquiterpen \\
\hline & 1542 & 22,056 & $?$ & & 0 & 0 & 0 & 0 & 0 & 0 & 4 & 0 & 0 & 0 & 0 & 0 & 0 & 0 & Unbekannt \\
\hline & 1545 & 22,102 & $?$ & & 0 & 0 & 0 & 0 & 0 & 0 & 0 & 4 & 0 & 0 & 0 & 4 & 0 & 0 & Unbekannt \\
\hline & 1551 & 22,201 & ? & & 0 & 0 & 0 & 0 & 0 & 0 & 0 & 4 & 0 & 0 & 0 & 0 & 0 & 0 & Unbekannt \\
\hline & 1557 & 22,287 & $?$ & & 0 & 0 & 0 & 0 & 0 & 0 & 0 & 4 & 0 & 0 & 0 & 0 & 0 & 0 & Unbekannt \\
\hline 1600 & 1598 & 22,946 & Hexadecan & 000544-76-3 & 0 & 0 & 0 & 0 & 3 & 0 & 0 & 3 & 2 & 5 & 5 & 5 & 0 & 0 & Alkan \\
\hline 1578 & 1606 & 23,065 & Diethylphthalat & $000084-66-2$ & 0 & 0 & 0 & 0 & 5 & 0 & 7 & 5 & 0 & 0 & 0 & 4 & 0 & 0 & Ester \\
\hline & 1672 & 24,054 & $6(\mathrm{E}), 8(\mathrm{E})$-Heptadecadien & \begin{tabular}{|l|}
$999281-78-6$ \\
\end{tabular} & 5 & 4 & 6 & 3 & 6 & 0 & 5 & 0 & 0 & 0 & 0 & 0 & 0 & 0 & Alken \\
\hline 1719 & 1680 & 24,166 & 8-Heptadecen & 002579-04-6 & 7 & 6 & 6 & 5 & 7 & 5 & 6 & 3 & 4 & 3 & 1 & 0 & 4 & 0 & Alken \\
\hline & 1686 & 24,259 & & & 0 & 0 & 0 & 4 & 0 & 0 & 3 & 2 & 0 & 0 & 0 & 4 & \begin{tabular}{c|c}
0 \\
\end{tabular} & & Unbekannt \\
\hline
\end{tabular}




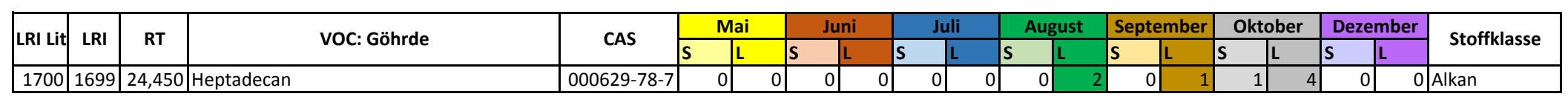

LRI Lit = linearer Retentionsindex in der Literatur; LRI = linearer Retetionsindex; VOC = flüchtige organische Verbindung; CAS = Chemical Abstracts Service Number; $S=$ Sand; $L=$ Lehm; $?=$ nicht identifiziertes VOC 


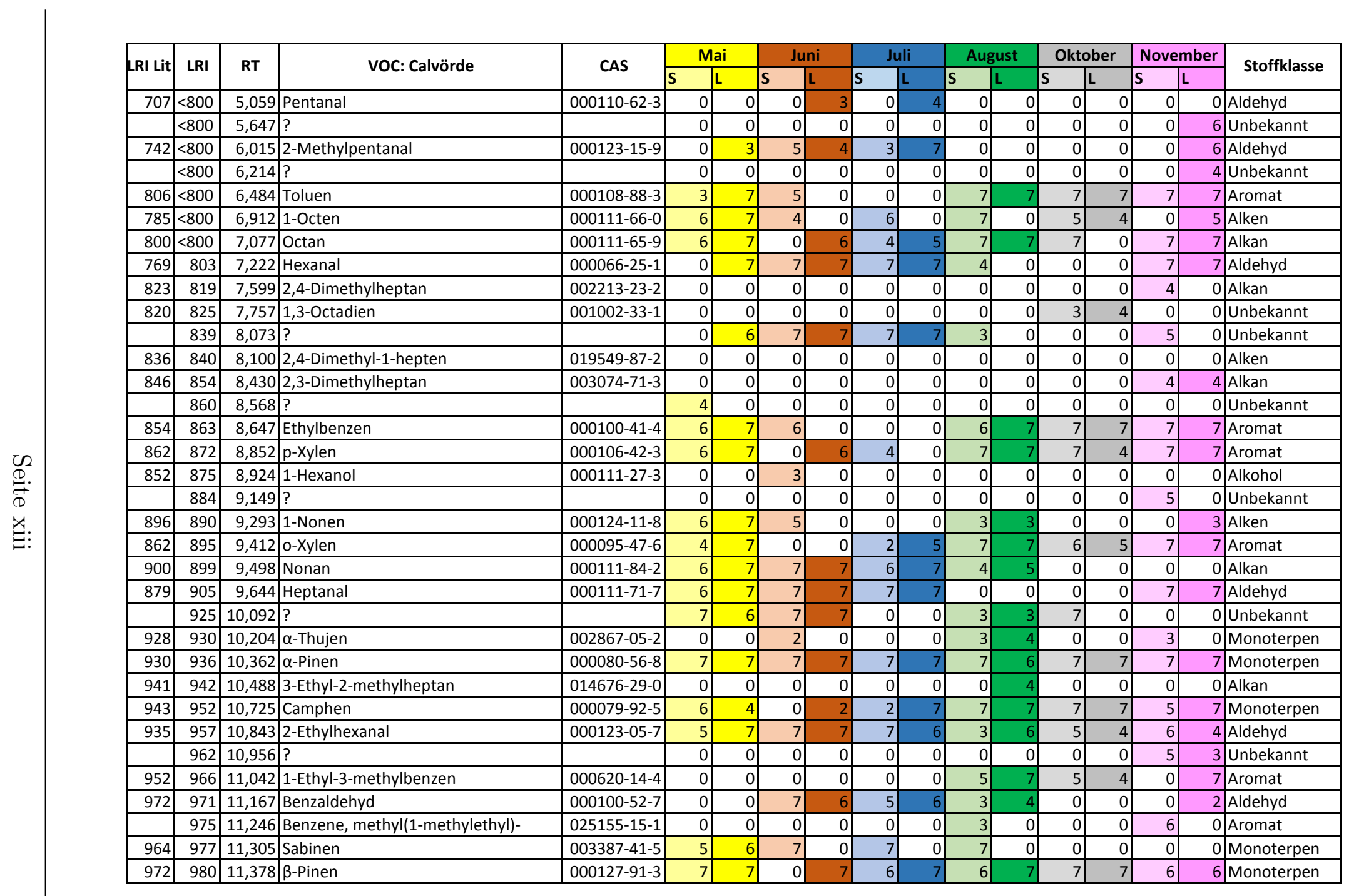




\begin{tabular}{|c|c|c|c|c|c|c|c|c|c|c|c|c|c|c|c|c|c|}
\hline \multirow{2}{*}{\begin{tabular}{|l|} 
LRI Lit \\
\end{tabular}} & \multirow{2}{*}{ LRI } & \multirow{2}{*}{ RT } & \multirow{2}{*}{ VOC: Calvörde } & \multirow{2}{*}{ CAS } & \multicolumn{2}{|c|}{ Mai } & \multicolumn{2}{|c|}{ Juni } & \multicolumn{2}{|c|}{ Juli } & \multicolumn{2}{|c|}{ August } & \multicolumn{2}{|c|}{ Oktober } & \multicolumn{2}{|c|}{ November } & \multirow{2}{*}{ Stoffklass } \\
\hline & & & & & $S$ & L & 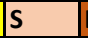 & L & S & $\mathrm{L}$ & $s$ & $\mathrm{~L}$ & s & $\mathrm{L}$ & \begin{tabular}{|l|l|l|l|l|}
$s$ & 1
\end{tabular} & $\mathrm{~L}$ & \\
\hline 988 & 985 & 11,484 & 1-Ethyl-2-methylbenzen & 000611-14-3 & 0 & 0 & 0 & 0 & 0 & 0 & 0 & 4 & 0 & 0 & 0 & 4 & \begin{tabular}{l|l}
4 & Aromat \\
\end{tabular} \\
\hline 962 & 989 & 11,589 & 3-Octanon & 000106-68-3 & 0 & 3 & 0 & 0 & 4 & 0 & 0 & 0 & 0 & 0 & 0 & 0 & Keton \\
\hline 996 & 992 & 11,648 & 2,2,4,6,6-Pentamethylheptan & 013475-82-6 & 0 & 0 & 6 & 0 & 2 & 0 & 7 & 7 & 0 & 5 & 0 & 7 & 7 Alkan \\
\hline 981 & 993 & 11,668 & $\beta$-Myrcen & 000123-35-3 & 0 & 0 & 2 & 0 & 6 & 0 & 0 & 0 & 0 & 0 & 7 & 0 & Monoterpen \\
\hline 978 & 997 & 11,774 & 1,2,4-Trimethylbenzen & 000095-63-6 & 0 & 0 & 0 & 0 & 0 & 0 & 0 & 0 & 6 & 3 & 6 & 6 & \begin{tabular}{|l|} 
Aromat \\
\end{tabular} \\
\hline 1000 & 999 & 11,819 & Decan & 000124-18-5 & 4 & 7 & 6 & 7 & 0 & 0 & 0 & 0 & 0 & 0 & 0 & 0 & \begin{tabular}{|l|l|}
0 Alkan \\
\end{tabular} \\
\hline 981 & 1005 & 11,945 & Octanal & 000124-13-0 & 6 & 7 & 7 & 6 & 7 & 7 & 5 & 0 & 0 & 0 & 3 & 5 & 5 Aldehyd \\
\hline 1005 & 1014 & 12,136 & 3-Caren & 013466-78-9 & 6 & 7 & 7 & 7 & 7 & 6 & 7 & 7 & 7 & 7 & 7 & 7 & 7 Monoterpen \\
\hline 1017 & 1020 & 12,268 & $\alpha$-Terpinen & 000099-86-5 & 0 & 0 & 4 & 0 & 2 & 0 & 0 & 4 & 0 & 0 & 0 & 0 & Monoterpen \\
\hline 1060 & 1023 & 12,334 & 4-Methyldecan & 002847-72-5 & 0 & 0 & 0 & 0 & 0 & 0 & 0 & 5 & 0 & 0 & 0 & 0 & Alkan \\
\hline 1029 & 1025 & 12,374 & 2,2,6-Trimethyloctan & 062016-28-8 & 0 & 0 & 0 & 0 & 0 & 0 & 0 & 0 & 0 & 0 & 6 & 0 & Alkan \\
\hline 1011 & 1028 & 12,453 & p-Cymol & 000099-87-6 & 5 & 7 & 7 & 7 & 7 & 7 & 6 & 7 & 7 & 7 & 0 & 7 & 7 Monoterpen \\
\hline 1019 & 1033 & 12,551 & Limonen & 000138-86-3 & 7 & 7 & 7 & 7 & 7 & 7 & 7 & 7 & 7 & 7 & 7 & 7 & 7 Monoterpen \\
\hline 1023 & 1037 & 12,631 & Eukalyptol & 000470-82-6 & 0 & 6 & 7 & 7 & 7 & 7 & 4 & 5 & 0 & 0 & 7 & 5 & 5 Monoterpen \\
\hline 1013 & 1041 & 12,717 & 2,6,6-Trimethylcyclohexanon & 002408-37-9 & 4 & 3 & 0 & 0 & 0 & 0 & 0 & 0 & 0 & 0 & 7 & 0 & Keton \\
\hline 1024 & 1045 & 12,809 & cis- $\beta$-Ocimen & $003338-55-4$ & 5 & 4 & 5 & 0 & 3 & 0 & 7 & 7 & 5 & 0 & 0 & 0 & Monoterpen \\
\hline & 1047 & 12,849 & ? & & 0 & 0 & 0 & 0 & 0 & 0 & 0 & 0 & 0 & 0 & 5 & 0 & Unbekannt \\
\hline & 1052 & 12,961 & $?$ & & 0 & 0 & 0 & 0 & 0 & 0 & 0 & 0 & 0 & 0 & 0 & 5 & 5 Unbekannt \\
\hline & 1055 & 13,020 & $?$ & & 0 & 0 & 0 & 0 & 0 & 0 & 4 & 0 & 7 & 0 & 7 & 0 & Unbekannt \\
\hline & 1057 & 13,067 & ? & & 0 & 0 & 0 & 0 & 0 & 0 & 0 & 0 & 0 & 0 & 0 & 5 & 5 Unbekannt \\
\hline & 1059 & 13,106 & ? & & 0 & 0 & 0 & 0 & 0 & 0 & 0 & 0 & 0 & 6 & 0 & 7 & 7 Unbekannt \\
\hline & 1059 & 13,112 & $?$ & & 0 & 0 & 7 & 0 & 6 & 5 & 6 & 0 & 0 & 0 & 5 & 0 & Unbekannt \\
\hline 1053 & 1063 & 13,204 & $\psi$-Terpinen & $000099-85-4$ & 0 & 0 & 7 & 7 & 5 & 7 & 2 & 7 & 0 & 0 & 0 & 0 & Monoterpen \\
\hline & 1065 & 13,238 & $?$ & & 0 & 0 & 0 & 0 & 0 & 0 & 0 & 0 & 0 & 0 & 7 & 5 & \begin{tabular}{|l|l|}
5 Unbekannt \\
\end{tabular} \\
\hline & 1068 & 13,317 & $?$ & & 0 & 0 & 0 & 0 & 0 & 0 & 0 & 5 & 0 & 4 & 0 & 0 & Unbekannt \\
\hline 1052 & 1078 & 13,521 & Acetophenon & $000098-86-2$ & 0 & 4 & 6 & 6 & 3 & 0 & 3 & 0 & 0 & 0 & 0 & 0 & \begin{tabular}{|l|} 
Keton \\
\end{tabular} \\
\hline & 1082 & 13,607 & $?$ & & 0 & 4 & 0 & 5 & 0 & 0 & 3 & 0 & 7 & 0 & 7 & 0 & Unbekannt \\
\hline & 1082 & 13,613 & ? & & 5 & 0 & 5 & 0 & 4 & 0 & 0 & 0 & 0 & 5 & 0 & 0 & Unbekannt \\
\hline & 1089 & 13,759 & ? & & 0 & 0 & 0 & 0 & 0 & 0 & 0 & 0 & 0 & 0 & 4 & 0 & Unbekannt \\
\hline & 1091 & 13,812 & $?$ & & 0 & 0 & 0 & 0 & 0 & 0 & 0 & 0 & 0 & 0 & 6 & 5 & 5 Unbekannt \\
\hline 1088 & 1092 & 13,825 & 1-Undecen & 000821-95-4 & 0 & 0 & 4 & 0 & 0 & 0 & 0 & 0 & 0 & 0 & 0 & 0 & Alken \\
\hline & 1096 & 13,917 & $?$ & & 0 & 0 & 0 & 0 & 0 & 0 & 4 & 0 & 0 & 0 & 7 & 4 & 4 Unbekannt \\
\hline & 1097 & 13,93 & $?$ & & 0 & 0 & 0 & 6 & 0 & 0 & 0 & 0 & 0 & 0 & 0 & 0 & Unbekannt \\
\hline 1100 & 1099 & 13,983 & Undecan & 001120-21-4 & 5 & 6 & 5 & 6 & 4 & 3 & 3 & 0 & 0 & 0 & 0 & 0 & Alkan \\
\hline
\end{tabular}




\begin{tabular}{|c|c|c|c|c|c|c|c|c|c|c|c|c|c|c|c|c|c|}
\hline \multirow{2}{*}{ LRI Lit } & \multirow{2}{*}{ LRI } & \multirow{2}{*}{ RT } & \multirow{2}{*}{ VOC: Calvörde } & \multirow{2}{*}{ CAS } & \multicolumn{2}{|c|}{ Mai } & \multicolumn{2}{|c|}{ Juni } & \multicolumn{2}{|c|}{ Juli } & \multicolumn{2}{|c|}{ August } & \multicolumn{2}{|c|}{ Oktober } & \multicolumn{2}{|c|}{ November } & \multirow{2}{*}{ Stoffklasse } \\
\hline & & & & & $S$ & L & $s$ & $\mathrm{TL}$ & $s$ & L & s & $\mathrm{L}$ & s & $\mathrm{L}$ & \begin{tabular}{|l|l|}
$s$ & \\
\end{tabular} & $\mathrm{~L}$ & \\
\hline & 1102 & 14,049 & $?$ & & 0 & 0 & 6 & 0 & 0 & 0 & 7 & 0 & 0 & 3 & 6 & & Unbekannt \\
\hline \multirow[t]{5}{*}{1128} & 1107 & 14,141 & Nonanal & 000124-19-6 & 6 & 7 & 7 & 7 & 7 & 7 & 7 & 7 & 5 & 1 & 7 & & Aldehyd \\
\hline & 1130 & 14,609 & $?$ & & 0 & 0 & 0 & 0 & 5 & 0 & 0 & 0 & 0 & 0 & 0 & & Unbekannt \\
\hline & 1135 & 14,715 & ? & & 0 & 0 & 0 & 0 & 5 & 0 & 0 & 0 & 0 & 0 & 0 & & Unbekannt \\
\hline & 1142 & 14,867 & ? & & 0 & 0 & 0 & 0 & 6 & 6 & 0 & 0 & 0 & 0 & 0 & 0 & Unbekannt \\
\hline & 1143 & 14,873 & $?$ & & 4 & 0 & 3 & 0 & 0 & 5 & 7 & 4 & 0 & 0 & 0 & 0 & Unbekannt \\
\hline 1146 & 1145 & 14,919 & 5-Ethyldecan & 017302-36-2 & 0 & 0 & 0 & 0 & 0 & 6 & 0 & 0 & 0 & 0 & 0 & & Alkan \\
\hline \multirow{3}{*}{1142} & 1147 & 14,966 & $(1 \mathrm{R})-(+)$-Norinon & 038651-65-9 & 6 & 0 & 7 & 7 & 6 & 6 & 0 & 0 & 0 & 0 & 0 & & Keton \\
\hline & 1148 & 14,982 & ? & & 0 & 6 & 0 & 0 & 0 & 0 & 0 & 0 & 0 & 0 & 0 & & Unbekannt \\
\hline & 1151 & 15,051 & $?$ & & 7 & 7 & 7 & 7 & 6 & 7 & 7 & 7 & 7 & 7 & 7 & & Unbekannt \\
\hline 1121 & 1154 & 15,104 & Campher & $000076-22-2$ & 6 & 6 & 7 & 7 & 7 & 0 & 4 & 0 & 7 & 4 & 0 & & Monoterpen \\
\hline \multirow{2}{*}{1126} & 1167 & 15,374 & Sabina ketone & 000513-20-2 & 0 & 3 & 7 & 7 & 4 & 0 & 0 & 0 & 0 & 0 & 0 & & \begin{tabular}{|l|} 
Keton \\
\end{tabular} \\
\hline & 1180 & 15,632 & $?$ & & 0 & 0 & 0 & 0 & 6 & 0 & 4 & 0 & 0 & 0 & 0 & & Unbekannt \\
\hline \multirow[t]{2}{*}{1165} & 1184 & 15,704 & 3,3,6-Trimethyldecan & 062338-14-1 & 0 & 0 & 0 & 0 & 0 & 6 & 0 & 0 & 0 & 0 & 0 & & Alkan \\
\hline & 1194 & 15,922 & ? & & 0 & 0 & 0 & 0 & 5 & 0 & 3 & 0 & 0 & 0 & 0 & & Unbekannt \\
\hline 1200 & 1199 & 16,008 & Dodecan & 000112-40-3 & 0 & 0 & 5 & 7 & 7 & 6 & 7 & 0 & 0 & 0 & 6 & & Alkan \\
\hline \multirow{2}{*}{1183} & 1208 & 16,186 & Decanal & 000112-31-2 & 6 & 4 & 7 & 6 & 7 & 7 & 5 & 0 & 4 & 0 & 2 & & Aldehyd \\
\hline & 1214 & 16,305 & $?$ & & 0 & 0 & 0 & 0 & 4 & 0 & 0 & 0 & 0 & 0 & 0 & 0 & Unbekannt \\
\hline 1196 & 1221 & 16,436 & Verbenon & 000080-57-9 & 0 & 0 & 6 & 5 & 4 & 0 & 6 & 0 & 0 & 0 & 0 & & Monoterpen \\
\hline 1210 & 1223 & 16,476 & 5-Propyldecan & 017312-62-8 & 0 & 0 & 0 & 0 & 0 & 6 & 0 & 0 & 0 & 0 & 0 & & Alkan \\
\hline \multirow{3}{*}{1224} & 1231 & 16,621 & 2-Ethylhexylpropenoat & 000103-11-7 & 4 & 0 & 0 & 0 & 5 & 0 & 4 & 0 & 0 & 0 & 0 & & Ester \\
\hline & 1247 & 16,918 & ? & & 0 & 0 & 0 & 0 & 0 & 0 & 0 & 7 & 0 & 0 & 0 & 0 & Unbekannt \\
\hline & 1254 & 17,056 & $?$ & & 0 & 0 & 0 & 0 & 7 & 0 & 0 & 0 & 0 & 0 & 0 & 0 & Unbekannt \\
\hline \multirow{2}{*}{1249} & 1259 & 17,162 & 1,3-Ditertiarybutylbenzen & 001014-60-4 & 0 & 0 & 0 & 0 & 0 & 0 & 4 & 0 & 0 & 0 & 0 & 0 & Aromat \\
\hline & 1280 & 17,558 & ? & & 0 & 0 & 7 & 6 & 4 & 0 & 7 & 0 & 0 & 0 & 6 & 6 & Unbekannt \\
\hline 1270 & 1293 & 17,808 & Bornylacetat & 000076-49-3 & 5 & 4 & 4 & 6 & 5 & 4 & 4 & 0 & 0 & 0 & 0 & 0 & Acetat \\
\hline \multirow{5}{*}{1300} & 1299 & 17,907 & Tridecan & $000629-50-5$ & 0 & 0 & 6 & 4 & 5 & 4 & 4 & 0 & 0 & 0 & 4 & 0 & Alkan \\
\hline & 1311 & 18,131 & $?$ & & 0 & 0 & 0 & 0 & 0 & 0 & 0 & 0 & 0 & 4 & 0 & 0 & Unbekannt \\
\hline & 1311 & 18,138 & ? & & 0 & 0 & 0 & 0 & 0 & 7 & 0 & 7 & 0 & 0 & 0 & 0 & Unbekannt \\
\hline & 1322 & 18,330 & ? & & 0 & 0 & 0 & 0 & 0 & 0 & 0 & 7 & 0 & 0 & 0 & 0 & Unbekannt \\
\hline & 1323 & 18,336 & $?$ & & 0 & 0 & 0 & 0 & 0 & 6 & 0 & 0 & 0 & 0 & 0 & 0 & Unbekannt \\
\hline \multirow[t]{3}{*}{1329} & 1328 & 18,429 & $2,2,4,4,6,8,8$-Heptamethylnonan & 004390-04-9 & 0 & 0 & 0 & 0 & 0 & 0 & 4 & 7 & 7 & 3 & 0 & 0 & Alkan \\
\hline & 1338 & 18,607 & ? & & 0 & 0 & 0 & 0 & 0 & 4 & 0 & 7 & 0 & 0 & 0 & 0 & Unbekannt \\
\hline & 1351 & 18,831 & $?$ & & 0 & 0 & 0 & 0 & 0 & 0 & 5 & 0 & 0 & 0 & 0 & & Unbekannt \\
\hline
\end{tabular}




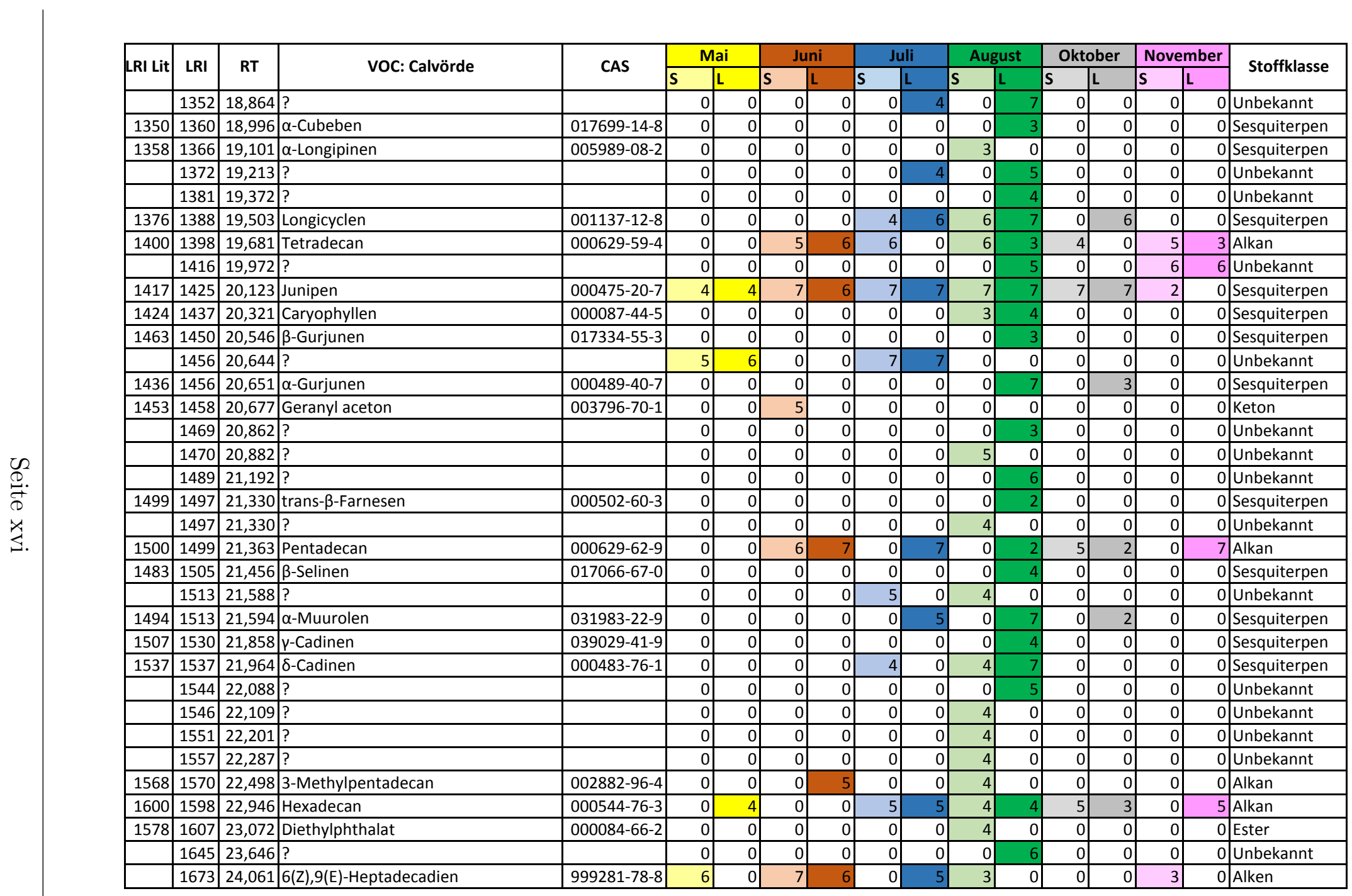




\begin{tabular}{|c|c|c|c|c|c|c|c|c|c|c|c|c|c|c|c|c|c|c|}
\hline \multirow{2}{*}{ LRI Lit } & \multirow{2}{*}{ LRI } & \multirow{2}{*}{ RT } & \multirow{2}{*}{ VOC: Calvörde } & \multirow{2}{*}{ CAS } & \multicolumn{2}{|c|}{ Mai } & \multicolumn{3}{|c|}{ Juni } & \multicolumn{2}{|c|}{ Juli } & \multicolumn{2}{|c|}{ August } & \multicolumn{2}{|c|}{ Oktober } & \multicolumn{2}{|c|}{ November } & \multirow{2}{*}{ Stoffklasse } \\
\hline & & & & & $\mathbf{S}$ & $\mathbf{L}$ & $S$ & $\mathrm{~L}$ & & S & L & $s$ & $\mathrm{~L}$ & $s$ & $\mathrm{~L}$ & \begin{tabular}{|l|l|}
$\mathbf{S}$ & \\
\end{tabular} & $\mathrm{L}$ & \\
\hline 1719 & 1680 & 24,173 & 8-Heptadecen & 002579-04-6 & 4 & 0 & & \begin{tabular}{l|l}
6 \\
\end{tabular} & 5 & 0 & 3 & 3 & 0 & 0 & 0 & 2 & & $\begin{array}{l}0 \text { Alken } \\
\end{array}$ \\
\hline 1700 & 1698 & 24,444 & Heptadecan & 000629-78-7 & 0 & 0 & t & 0 & 0 & 0 & 0 & 3 & 0 & 4 & 0 & 0 & & \begin{tabular}{l|l} 
Alkan \\
\end{tabular} \\
\hline
\end{tabular}

LRI Lit = linearer Retentionsindex in der Literatur; LRI = linearer Retetionsindex; VOC = flüchtige organische Verbindung; CAS = Chemical Abstracts Service Number; $S=$ Sand; $L=$ Lehm; $?=$ nicht identifiziertes $\mathrm{VOC}$ 
Table A.5: Signifikante Unterschiede in den Konzentrationen verschiedener VOC in Bezug auf die Einflussfaktoren

\begin{tabular}{|l|l|l|l|l|}
\hline \multicolumn{5}{|c|}{ p-Wert } \\
\hline VOC & CAS & Untersuchungsgebiet & Bodentyp & Jahreszeit \\
\hline \hline$\alpha$-Pinen & $000080-56-8$ & $<0,001^{* * *}$ & n. s. & n. s. \\
\hline Limonen & $000138-86-3$ & $0,030^{*}$ & n. s. & $<0,001^{* * *}$ \\
\hline Camphen & $000079-92-5$ & n. s. & n. s. & n. s. \\
\hline Camphor & $000076-22-2$ & $<0,001^{* * *}$ & $0,009^{* *}$ & $<0,001^{* * *}$ \\
\hline Nonanal & $000124-19-6$ & n. s. & $0,030^{*}$ & $<0,001^{* * *}$ \\
\hline Decanal & $000112-31-2$ & n. s. & $0,005^{* *}$ & $<0,001^{* * *}$ \\
\hline
\end{tabular}

ANOVA: $* \mathrm{p} \leq 0,05, * * \mathrm{p} \leq 0,01, * * * \mathrm{p} \leq 0,001, \mathrm{n} . \mathrm{s} .=$ nicht signifikant

Table A.6: Signifikante Abhängigkeit der Konzentrationen von sechs VOC bei drei Einflussfaktoren

\begin{tabular}{|l|l|l|l|l|l|l|l|}
\hline \multicolumn{1}{|c|}{ p-Wert } \\
\hline \multirow{3}{*}{ Faktor } & VOC & $\alpha$-Pinen & Limonen & Nonanal & Decanal & Camphen & Camphor \\
\cline { 2 - 8 } & U vs G & $<0,001^{* * *}$ & n. s. & n. s. & n. s. & n. s. & $0,005^{* *}$ \\
\hline \multirow{2}{*}{ Bodentyp } & San vs C & $<0,001^{* * *}$ & $0,050^{*}$ & n. s. & n. s. & n. s. & n. s. \\
\hline Jahreszeit & F vs S & n. s. & n. s. & $<0,001^{* * *}$ & $0,026^{*}$ & n. s. & n. s. \\
\cline { 2 - 8 } & F vs H & n. s. & $0,002^{* *}$ & $<0,001^{* * *}$ & $<0,001^{* * *}$ & n. s. & $<0,001^{* * *}$ \\
\cline { 2 - 8 } & F vs W & n. s. & $0,002^{* *}$ & $<0,001^{* * *}$ & $<0,001^{* * *}$ & n. s. & $<0,001^{* * *}$ \\
\cline { 2 - 8 } & S vs H & n. s. & $0,050^{*}$ & n. s. & n. s. & n. s. & $0,012^{*}$ \\
\cline { 2 - 8 } & S vs W & n. s. & $0,042^{*}$ & n. s. & $0,006^{* *}$ & n. s. & $<0,001^{* * *}$ \\
\cline { 2 - 8 } & H vs W & n. s. & n. s. & n. s. & n. s. & n. s. & $0,016^{*}$ \\
\hline
\end{tabular}

Bonferroni T-Test: ${ }^{*} \mathrm{p} \leq 0,05,{ }^{* *} \mathrm{p} \leq 0,01,{ }^{* * *} \mathrm{p} \leq 0,001, \mathrm{n} . \mathrm{s} .=$ nicht signifikant. $\mathrm{U}=$ Unterlüß, $\mathrm{G}=$ Göhrde, $\mathrm{C}=$ Calvörde, $\mathrm{F}=$ Frühling, $\mathrm{S}=$ Sommer, $\mathrm{H}=$ Herbst, $\mathrm{W}=$ Winter 


\section{A.3. Wurzel-VOC von $F$. sylvatica}

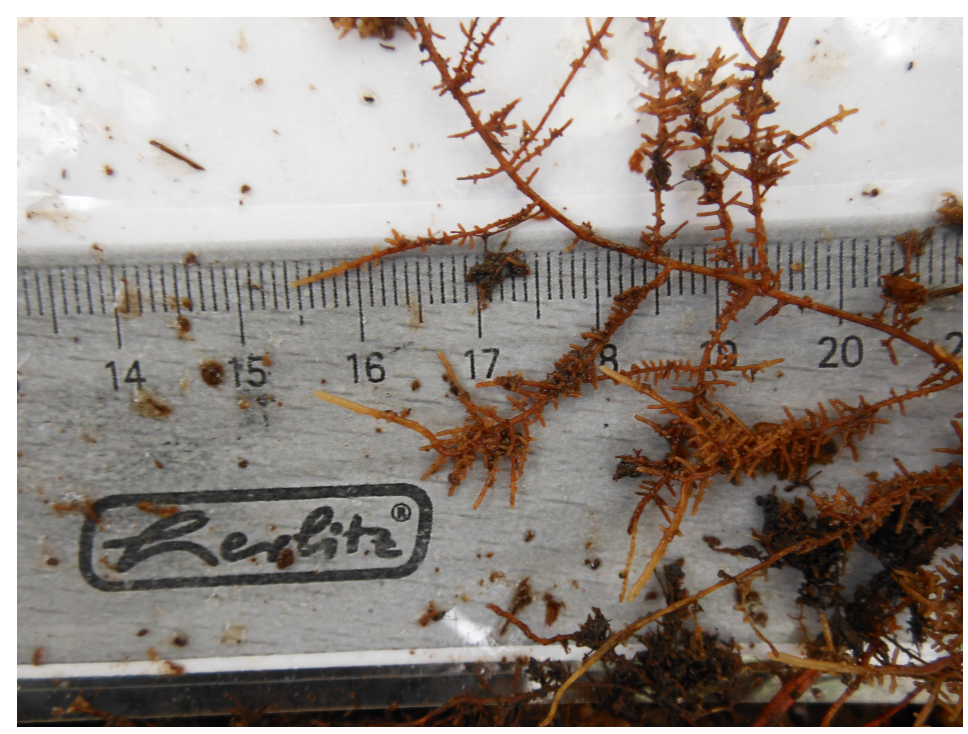

Figure A.1: Feinwurzel mit Mykorrhizierung

Table A.7: Klimadaten für die Wurzel-VOC-Messungen im Mai

\begin{tabular}{|l|c|r|r|c|}
\hline Fläche & Datum & mittl. Temperatur & mittl. rel. Luftfeuchte & Messzeit \\
\hline Sellhorn & 07.05 .2015 & $11,3{ }^{\circ} \mathrm{C}$ & $70,2 \%$ & $10: 41-12: 41 \mathrm{~h}$ \\
\hline Unterlüß & 05.05 .2015 & $18,2{ }^{\circ} \mathrm{C}$ & $88,7 \%$ & $12: 24-14: 24 \mathrm{~h}$ \\
\hline Klötze & 12.05 .2015 & $25,3{ }^{\circ} \mathrm{C}$ & $47,1 \%$ & $12: 25-14: 25 \mathrm{~h}$ \\
\hline
\end{tabular}


Table A.8: Wurzellänge der Feinwurzeln im Oktober 2013

\begin{tabular}{|l|c|c|r|}
\hline Standort & Baumnr. & Grube & Wurzellänge [cm] \\
\hline Sellhorn & 1 & 1 & 35 \\
\hline Sellhorn & 2 & 1 & 40 \\
\hline Sellhorn & 3 & 1 & 40 \\
\hline Sellhorn & 4 & 2 & 50 \\
\hline Sellhorn & 5 & 2 & 50 \\
\hline Sellhorn & 6 & 2 & 60 \\
\hline Sellhorn & 7 & 3 & 45 \\
\hline Sellhorn & 8 & 3 & 45 \\
\hline Sellhorn & 9 & 3 & 25 \\
\hline Unterlüß & 1 & 1 & 100 \\
\hline Unterlüß & 2 & 1 & 160 \\
\hline Unterlüß & 3 & 1 & 80 \\
\hline Unterlüß & 4 & 2 & 40 \\
\hline Unterlüß & 5 & 2 & 80 \\
\hline Unterlüß & 6 & 2 & 40 \\
\hline Unterlüß & 7 & 3 & 25 \\
\hline Unterlüß & 8 & 3 & 25 \\
\hline Unterlüß & 9 & 3 & 60 \\
\hline Klötze & 1 & 1 & 110 \\
\hline Klötze & 2 & 1 & 100 \\
\hline Klötze & 3 & 1 & 45 \\
\hline Klötze & 4 & 2 & 70 \\
\hline Klötze & 5 & 2 & 45 \\
\hline Klötze & 6 & 2 & 70 \\
\hline Klötze & 7 & 3 & 30 \\
\hline Klötze & 8 & 3 & 3 \\
\hline Klötze & 9 & 3 & \\
\hline & & & 2 \\
\hline
\end{tabular}


Table A.9: Wurzellänge, Abstand zum jeweiligen Baum und BHD für die VOC-Messung der Feinwurzeln im Mai 2015

\begin{tabular}{|l|r|r|r|r|}
\hline Gebiet & Baumnr. & Wurzellänge [cm] & Abstand zum Baum [m] & BHD [cm] \\
\hline Sellhorn & 1 & 40 & 4,00 & 102 \\
\hline Sellhorn & 2 & 38 & 2,48 & 141 \\
\hline Sellhorn & 3 & 39 & 2,53 & 150 \\
\hline Sellhorn & 4 & 34 & 1,66 & 131 \\
\hline Sellhorn & 5 & 45 & 1,93 & 136 \\
\hline Sellhorn & 6 & 39 & 2,34 & 152 \\
\hline Sellhorn & 7 & 35 & 2,00 & 105 \\
\hline Sellhorn & 8 & 30 & 2,16 & 151 \\
\hline Sellhorn & 9 & 40 & 3,60 & 192 \\
\hline Unterlüß & 1 & 48 & 2,85 & 168 \\
\hline Unterlüß & 2 & 54 & 2,27 & 148 \\
\hline Unterlüß & 3 & 29 & 2,15 & 106 \\
\hline Unterlüß & 4 & 52 & 2,24 & 148 \\
\hline Unterlüß & 5 & 46 & 1,98 & 100 \\
\hline Unterlüß & 6 & 51 & 2,33 & 113 \\
\hline Unterlüß & 7 & 46 & 2,56 & 127 \\
\hline Unterlüß & 8 & 34 & 2,28 & 147 \\
\hline Unterlüß & 9 & 39 & 1,87 & 163 \\
\hline Klötze & 1 & 43 & 2,80 & 136 \\
\hline Klötze & 2 & 57 & 1,85 & 141 \\
\hline Klötze & 3 & 49 & 1,75 & 188 \\
\hline Klötze & 4 & 25 & 1,90 & 129 \\
\hline Klötze & 5 & 23 & 3,20 & 157 \\
\hline Klötze & 6 & 50 & 1,65 & 134 \\
\hline Klötze & 7 & 47 & 1,90 & 176 \\
\hline Klötze & 8 & 25 & 2,80 & 199 \\
\hline Klötze & 9 & & & 140 \\
\hline
\end{tabular}


A.4. Zuordnung der Wurzel-VOC von F. sylvatica zu weiteren Produzenten 
Tabelle: Zuordnung der Wurzel-VOC zu den Produzenten

\begin{tabular}{|c|c|c|c|c|c|c|}
\hline \multirow[b]{2}{*}{$\mathbf{L R I}^{1}$} & \multirow[b]{2}{*}{ VOC } & \multicolumn{5}{|c|}{ Produzent } \\
\hline & & Pflanze & Pilz & $\begin{array}{l}\text { Lebens } \\
\text { zyklus }\end{array}$ & Stamm & Bakterien \\
\hline$<700$ & Acetic acid" & & Tuber excavatum $[28]$ & $\mathrm{M}$ & $\mathrm{A}$ & \\
\hline \multirow[t]{2}{*}{766} & 1-Pentanol ${ }^{\#}$ & Hordeum vulgare $[18]$ & $\begin{array}{l}\text { Trichoderma spp. [45], [29]; Tuber indicum [38] } \\
\text { Fusarium graminearum [7] }\end{array}$ & $\begin{array}{l}\mathrm{M} \\
\mathrm{P}\end{array}$ & $\begin{array}{l}\mathrm{A} \\
\mathrm{A}\end{array}$ & $\begin{array}{l}\text { Bacillus spp. [25], [15] } \\
\text { Escherichia coli [15] }\end{array}$ \\
\hline & & & Armillaria mellea [29] & & & \\
\hline \multirow[t]{7}{*}{805} & Hexanal $\left.\right|^{* * \times}$ & Smyrnium olusatrum [3] & $\begin{array}{l}\text { Trichoderma spp. [45]; Tuber spp. [reviewed by 39], [28], } \\
\text { [12] }\end{array}$ & M & A & \\
\hline & & Trifolium pratense [31] & Agaricus bisporus [19] & $\mathrm{S}$ & B & \\
\hline & & Festuca pratensis $\mathrm{x}$ Lolium perenne [34] & Fusarium graminearum [7] & $\mathrm{P}$ & A & \\
\hline & & Hordeum vulgare $[18]$ & Gymnopilus penetrans [8] & $\mathrm{s}$ & B & \\
\hline & & Hordeum distichon [5] & Cenococcum geophilum $[8]$ & M & A & \\
\hline & & Eryngium palmatum [27] & Sillus varigatus $[8]$ & M & B & \\
\hline & & & Meliniomyces variables [8]; Phialocephala fortinii [8] & M & A & \\
\hline 810 & 3-Cyclohepten-1-on & & Paxillus involutus [29] & $\mathrm{M}$ & $\mathrm{B}$ & \\
\hline 858 & (Z)-3-Hexen-1-ol ${ }^{\#}$ & Hordeum vulgare $[18]$ & & & & \\
\hline 863 & 4-Methyloctan ${ }^{\#}$ & & & & & \\
\hline 864 & Ethylbenzol $^{1 \times}$ & & Tuber aestivum [12] & $\mathrm{M}$ & A & \\
\hline 888 & Heptanon $^{\#}$ & & & & & \\
\hline 896 & $\begin{array}{l}y l e n^{\# x} \\
\end{array}$ & & $\begin{array}{l}\text { Trichoderma spp. [45] } \\
\text { Fusarium graminearum [7] }\end{array}$ & $\mathrm{M}$ & $\mathrm{A}$ & \\
\hline 901 & Nonan $^{\# x}$ & & Fusarium graminearum [7] & & $\mathrm{A}$ & Pseudomonas spp. [16] \\
\hline \multirow[t]{2}{*}{906} & Heptanal $^{\# \times}$ & Eryngium palmatum [27] & Trichoderma spp. [45]; Tuber spp. [12], [28] & $\mathrm{M}$ & A & \\
\hline & & & Fusarium graminearum $[7]$ & $\mathrm{P}$ & A & \\
\hline 920 & Anisol & Quercus spp. [47] & & & & Chondromyces crocatus [37] \\
\hline \multirow[t]{7}{*}{937} & $\alpha$-Pinen ${ }^{\# x}$ & Pinus pinea [26] & Fomes fomentarius [17] & $\mathrm{S} ; \mathrm{P}$ & B & \\
\hline & & Smyrnium olusatrum [3] & Gloephyllum trabeum [42]; Trametes versicolor [42]; & S & B & \\
\hline & & Trifolium pratense [31] & Tyromyces placenta $[42]$ & s & D & \\
\hline & & Artemisia tridentata $[21]$ & Fusarium graminearum [7] & $\mathrm{P}$ & A & \\
\hline & & Daucus carota ssp. sativus [46] & Gymnopilus penetrans $[8]$ & $\mathrm{s}$ & B & \\
\hline & & Quercus sp. [46] & Piloderma olivaceum [8] & M & B & \\
\hline & & Pseudotsuga menziesii [23] & Tomentellopsis submollis [8] & $\mathrm{M}$ & A & \\
\hline 952 & Camphen $^{\# x}$ & $\begin{array}{l}\text { Pinus pinea [26]; Valeriana officinalis [33] } \\
\text { Artemisia tridentata [21] } \\
\text { Pseudotsuga menziesii [23] }\end{array}$ & & & & \\
\hline 958 & 2-Ethylhexanal ${ }^{\text {fx }}$ & & & & & \\
\hline \multirow[t]{5}{*}{974} & ${\text { Benzaldehyd }{ }^{\sharp x}}$ & & $\begin{array}{l}\text { Trichoderma spp. [45]; Tuber spp. [28], [12] } \\
\end{array}$ & $\mathrm{M}$ & A & Escherichia coli $[15]$ \\
\hline & & & Fomes fomentarius [20], [17] & $\mathrm{S} ; \mathrm{P}$ & B & Pseudomonas spp. [16] \\
\hline & & & Agaricus bisporus [19]; Bjerkandera adusta [24] & $\mathrm{s}$ & B & Bacillus spp. [48], [25], [15] \\
\hline & & & Paecilomyces lilacinus [48]; Pochonia chlamydosporia [48] & $\mathrm{S}$ & A & \\
\hline & & & Fusarium graminearum $[7]$ & $\mathrm{P}$ & A & \\
\hline
\end{tabular}


Tabelle: Zuordnung der Wurzel-VOC zu den Produzenten (Fortsetzung)

\begin{tabular}{|c|c|c|c|c|c|c|}
\hline \multirow[b]{2}{*}{ LRI $^{1}$} & \multirow[b]{2}{*}{ VOC } & \multicolumn{5}{|c|}{ Produzent } \\
\hline & & Pflanze & Pilz & $\begin{array}{l}\text { Lebens } \\
\text { zyklus }\end{array}$ & Stamm & Bakterien \\
\hline \multirow[t]{14}{*}{981} & 1-Octen-3-o ${ }^{* *}$ & Quercus spp. [47] & Penicillium [6], [9], [22] & $\mathrm{s}$ & A & \\
\hline & & Hordeum vulgare $[18]$ & Paxillus involutus $[29]$ & M & B & \\
\hline & & & Typischer Pilzduft [10] & & & \\
\hline & & & Trichoderma spp. [30], [29],[11] & M & A & \\
\hline & & & Fomes fomentarius $[20]$ & $\mathrm{S} ; \mathrm{P}$ & B & \\
\hline & & & $\begin{array}{l}\text { Agaricus bisporus [1]; Gloephyllum trabeum [42]; Trametes } \\
\text { versicolor [42]: Poria placenta [42]: Armillaria mellea [29] }\end{array}$ & $\mathrm{s}$ & B & \\
\hline & & & $\begin{array}{l}\text { Aspergillus spp. [22], [reviewed by 41] } \\
\text { S }\end{array}$ & $\mathrm{S} ; \mathrm{P}$ & A & \\
\hline & & & Alternaria [22] & $\mathrm{S} ; \mathrm{P}$ & A & \\
\hline & & & Fusarium [22], [7] & $\mathrm{P}$ & A & \\
\hline & & & Tuber spp. [32], [12], [28], [reviewed by 39] & M & A & \\
\hline & & & Gymnopilus penetrans $[8]$ & $\mathrm{s}$ & B & \\
\hline & & & Cenococcum geophilum $[8]$ & M & A & \\
\hline & & & Piloderma olivaceum [8]; Sillus varigatus [8] & M & B & \\
\hline & & & Meliniomyces variables $[8]$, Phialocephala fortinii $[8]$ & $\mathrm{M}$ & A & \\
\hline \multirow[t]{9}{*}{988} & 3-Octanon ${ }^{* *}$ & $\begin{array}{l}\text { Trifolium pratense [31] } \\
\end{array}$ & Paxillus involutus [29] & $\mathrm{M}$ & $\mathrm{B}$ & \\
\hline & & Quercus spp. [47] & Typischer Pilzduft [10] & & & \\
\hline & & & $\begin{array}{l}\text { Trichoderma spp. [30], [11], [29]; Tuber spp. [reviewed by } \\
\text { 39], [28], [12], [32], [43] }\end{array}$ & M & A & \\
\hline & & & Fomes fomentarius $[20],[17]$ & $\mathrm{S} ; \mathrm{P}$ & B & \\
\hline & & & $\begin{array}{l}\text { Agaricus bisporus [19]; Gloephyllum trabeum [42]; Trametes } \\
\text { versicolor [42]: Poria placenta [l2]: Armillaria mellea [29] }\end{array}$ & $\mathrm{S}$ & B & \\
\hline & & & Aspergillus spp. [22], [reviewed by 40] & $\mathrm{S} ; \mathrm{P}$ & A & \\
\hline & & & Penicillium spp. [22], [9] & s & A & \\
\hline & & & Alternaria [22] & $\mathrm{S} ; \mathrm{P}$ & A & \\
\hline & & & Fusarium [22] & $\mathrm{P}$ & A & \\
\hline \multirow[t]{4}{*}{997} & 3-Octanol ${ }^{\#}$ & & Typischer Pilzduft [10] & & & \\
\hline & - Cotanor & & Aspergillus flavus [reviewed by 40] & $\mathrm{P}$ & A & \\
\hline & & & Penicillium paneum $[9]$ & S & A & \\
\hline & & & Tuber borchii [38]; Trichoderma spp. [30] & M & A & \\
\hline 1000 & Decan $^{*}$ & & Tuber spp. [28] & $\mathrm{M}$ & $\overline{\mathrm{A}}$ & $\begin{array}{l}\text { Pseudomonas spp. [16] } \\
\text { Bacillus spp. subtilis [35], [36] }\end{array}$ \\
\hline \multirow[t]{2}{*}{1006} & Octanal $^{+x}$ & Eryngium palmatum [27] & & $\mathrm{S} ; \mathrm{P}$ & $\mathrm{B}$ & \\
\hline & & & $\begin{array}{l}\text { Tuber aestivum }[12] \\
\end{array}$ & $\mathrm{M}$ & $\mathrm{A}$ & \\
\hline \multirow[t]{3}{*}{1014} & 3 -Caren ${ }^{\# x}$ & Smyrnium olusatrum [3] & Gloephyllum trabeum [42]; Trametes versicolor [42] & $\mathrm{S}$ & $\mathrm{B}$ & \\
\hline & & Quercus sp. [46] & Fusarium graminearum [7] & $\mathrm{P}$ & A & \\
\hline & & Chrysanthemoides monilifera [13] & & & & \\
\hline 1023 & 3-Cyclohexen-1-carbonitril ${ }^{\#}$ & & & & & \\
\hline 1031 & 2-Ethylhexanol ${ }^{* * x}$ & & Tuber aestivum [12] & $\overline{\mathrm{M}}$ & A & Pseudomonas spp. [16] \\
\hline & & Daucus carota ssp. sativus [46] & & & & \\
\hline
\end{tabular}


Tabelle: Zuordnung der Wurzel-VOC zu den Produzenten (Fortsetzung)

\begin{tabular}{|c|c|c|c|c|c|c|}
\hline \multirow[b]{2}{*}{ LRI $^{1}$} & \multirow[b]{2}{*}{ vOC } & \multicolumn{5}{|c|}{ Produzent } \\
\hline & & Pflanze & Pilz & $\begin{array}{l}\text { Lebens } \\
\text { zyklus }\end{array}$ & Stamm & Bakterien \\
\hline \multirow[t]{5}{*}{1034} & Limonen $^{\# x}$ & Pinus pinea $[26]$ & Trichoderma spp. [45], [30]; Tuber brumale [28] & $\mathrm{M}$ & A & \\
\hline & & Smyrnium olusatrum [3] & Fomes fomentarius [17] & $\mathrm{S} ; \mathrm{P}$ & B & \\
\hline & & Trifolium pratense [31] & Gymnopilus penetrans $[8]$ & $\mathrm{S}$ & B & \\
\hline & & Daucus carota ssp. sativus [46] & Cenococcum geophilum [8] & M & A & \\
\hline & & Pseudotsuga menziesii $[23]$ & Piloderma olivaceum $[8]$ & $\mathrm{M}$ & B & \\
\hline 1096 & 1-Phenylethanon ${ }^{*}$ & & Bjerkandera adusta [24] & $\mathrm{s}$ & $\mathrm{B}$ & \\
\hline 1094 & 2-Nonanon ${ }^{*}$ & Eryngium palmatum [27] & $\begin{array}{l}\text { Muscodor albus [41], [14] } \\
\text { Trichoderma spp. [11] }\end{array}$ & $\begin{array}{l}\mathrm{S} \\
\mathrm{M}\end{array}$ & $\begin{array}{l}\mathrm{A} \\
\mathrm{A}\end{array}$ & $\begin{array}{l}\text { Staphylococcus pasteuri [2] } \\
\text { Burkholderia spp. [4] } \\
\text { Serratia spp. [4] } \\
\text { Pseudosomas spp. [4] } \\
\text { Escherichia coli [4] }\end{array}$ \\
\hline 1100 & Undecan ${ }^{\#}$ & & Fusarium graminearum [7] & $P$ & $\overline{\mathrm{A}}$ & $\begin{array}{l}\text { Pseudomonas spp. [16], [15], [4] } \\
\text { Bacillus spp. [25], [15], [35], [36] } \\
\text { Paenibacillus polymyxa [25] } \\
\text { Escherichia coli [15] } \\
\end{array}$ \\
\hline \multirow[t]{3}{*}{1106} & Nonanal $^{* * x}$ & $\begin{array}{l}\text { Quercus spp. [47], [46] } \\
\text { Malus domestica [1] }\end{array}$ & $\begin{array}{l}\text { Trichoderma spp. [45]; Tuber spp. [12] } \\
\text { Fomes fomentarius [17] }\end{array}$ & $\begin{array}{l}\mathrm{M} \\
\mathrm{S} ; \mathrm{P}\end{array}$ & $\begin{array}{l}\text { A } \\
\text { B }\end{array}$ & Pseudomonas spp. $[16]$ \\
\hline & & Eryngium palmatum [27] & Fusarium graminearum [7] & $\mathrm{P}$ & A & \\
\hline & & & Penicillium paneum [9] & $\mathrm{s}$ & A & \\
\hline 1154 & Camphor $^{*}$ & $\begin{array}{l}\text { Artemisia tridentata }[21] \\
\text { Quercus spp. [47], }[46] \\
\text { Malus domestica }[1] \\
\text { Festuca pratensis x Lolium perenne [34] }\end{array}$ & & & & \\
\hline 1171 & 2-Nonenal ${ }^{*}$ & $\begin{array}{l}\text { Eryngium palmatum }[27] \\
\text { Hordeum vulgare }[18]\end{array}$ & Fomes fomentarius [20] & $\mathrm{S} ; \mathrm{P}$ & $\mathrm{B}$ & \\
\hline 1169 & Benzolcarbonsäure & & Bjerkandera adusta [24] & $\mathrm{S}$ & $\mathrm{B}$ & \\
\hline 1197 & Naphthalen $^{*}$ & & Fusarium graminearum [7] & $\mathrm{P}$ & A & \\
\hline \multirow[t]{3}{*}{1200} & Dodecan $^{*}$ & & Fusarium graminearum [7] & $P$ & A & Paenibacillus polymyxa [25] \\
\hline & & & & & & Escherichia coli [15] \\
\hline & & & & & & Bacillus spp. [48], [25], [35], [15] \\
\hline \multirow{3}{*}{1210} & Decanal $^{+}$ & Ouercus spp. [47], [46] & Trichoderma spp. [45] & $\bar{M}$ & $\bar{A}$ & $\begin{array}{l}\text { Pseudomonas spp. [15], [16] } \\
\text { Pseudomonas spp. [16] }\end{array}$ \\
\hline & & Malus domestica [1] & Fusarium graminearum [7] & $\mathrm{P}$ & A & Bacillus spp. subtilis [35]. [36] \\
\hline & & & Penicillium paneum $[9]$ & $\mathrm{S}$ & A & Burkholderia hospita [4] \\
\hline 1265 & Caprolactam ${ }^{\#}$ & & & & & \\
\hline \multirow[t]{3}{*}{1295} & 2-Undecanon ${ }^{\#}$ & & Trichoderma spp. [11] & M & A & \\
\hline & & & & & & Bacillus subtilis [35]; Serratia spp. [44] \\
\hline & & & & & & $\begin{array}{l}\text { Staphylococcus pasteuri [2] } \\
\text { Burkholderia spp.; Escherichia coli [4] }\end{array}$ \\
\hline
\end{tabular}


Tabelle: Zuordnung der Wurzel-VOC zu den Produzenten (Fortsetzung)

\begin{tabular}{|c|c|c|c|c|c|c|}
\hline \multirow[b]{2}{*}{$\mathbf{L R I}^{1}$} & \multirow[b]{2}{*}{ VOC } & \multicolumn{5}{|c|}{ Produzent } \\
\hline & & Pflanze & Pilz & Lebens & Stamm & Bakterien \\
\hline 1300 & Tridecan $^{\#}$ & & & & & Paenibacillus polymyxa [25] \\
\hline 1310 & Undecanal ${ }^{*}$ & & & & & Serratia spp. [4] \\
\hline 1400 & Tetradecan ${ }^{\#}$ & & $\begin{array}{l}\text { Fusarium graminearum }[7] \\
\text { Tuber borchii }[28]\end{array}$ & $\begin{array}{l}\mathrm{P} \\
\mathrm{M}\end{array}$ & $\begin{array}{l}\mathrm{A} \\
\mathrm{A}\end{array}$ & Psendomonas sp. $[16]$ \\
\hline 1500 & Pentadecan $^{\# \times}$ & Chrysanthemoides monilifera [13] & $\begin{array}{l}\text { Trichoderma spp. [45] } \\
\text { Fusarium graminearum [7] }\end{array}$ & $\begin{array}{l}\mathrm{M} \\
\mathrm{P}\end{array}$ & $\begin{array}{l}\text { A } \\
\text { A }\end{array}$ & $\begin{array}{l}\text { Pseudomonas spp. }[16] \\
\text { Burkholderia sachari }[4] \\
\text { Serratia entomophilia }[4]\end{array}$ \\
\hline 1600 & Hexadecan ${ }^{\text {\#x }}$ & & Fusarium graminearum [7] & $P$ & $\mathrm{~A}$ & $\begin{array}{l}\text { Bacillus spp. subtilis [15] } \\
\text { Escherichia coli [15] } \\
\text { Pseudomonas spp. [15], [16] }\end{array}$ \\
\hline
\end{tabular}

${ }^{1}$ Linearer Retentionsindex; * = Dominate VOC in den Chromatogrammen der Wurzelproben; ${ }^{\#}=$ durch Koelution mit authentischen Standardsubstanzen bestätigt

$\times=$ Rot-Buchenstamm-VOC; Lebenszyklus: M= Mykorrhiza, P= Pathogen, S= Saprophyt; Stamm: A= Ascomycota, B= Basidiomycota

\section{Literatur:}

Abraham, J.; Giacomuzzi, V.; Sergio, A.: Root damage to apple plants by cockchafer larvae induces a change in volatile signals below- and aboveground. In: Entomol. Exp. Appl. 156 (2015), Nr. 3, S. 279-289

2. Barbieri, E.; Gioacchini, A. M.; Zambonelli, A.; Bertini, L.; Stocchi, V.: Determination of microbial volatile organic compounds from Staphylococcus pasteuri against Tuber borchii using solid-phase microextraction and gas chromatography/ion trap mass spectrometry. In: Rapid Commun. Mass Spectrom. 19 (2005), Nr. 22, S. 3411-3415

3. Bertoli, A.; Pistelli, L.; Morelli, I.; Fraternale, D.; Giamperi, L.; Ricci, D.: Volatile constituents of different parts (roots, stems and leaves) of Smyrnium olusatrum L. In: Flavour Fragr. J. 19 (2004), Nr. 6, S. 522-525

4. Blom, D.; Fabbri, C.; Connor, E. C.; Schiestl, F. P.; Klauser, D. R.; Boller, T.; Eberl, L.; Weisskopf, L.: Production of plant growth modulating volatiles is widespread among rhizosphere bacteria and strongly depends on culture conditions. In: Environ. Microbiol. 13 (2011), Nr. 11, S.3047-3058

5. Barsics, F.; Delory, B. M.; Delaplace, P.; Francis, F.; Fauconnier, M. L.; Haubruge, É.; Verheggen, F. J.: Foraging wireworms are attracted to root-produced volatile aldehydes. In: J. Pest Sci. 90 (2017), Nr. 1, S. 69-76

6. Bjurman, J.; Nordstrand, E.; Kristensson, J.: Growth-phase-related production of potential volatile-organic tracer compounds by moulds on wood. In: Indoor Air. 7 (1997), Nr. 1, S. 2-7

7. Buśko, M.; Kulik, T.; Ostrowska, A.; Góral, T.; Perkowski, J.: Quantitative volatile compound profiles in fungal cultures of three different Fusarium graminearum chemotypes. In: FEMS Microbiol. Lett. 359 (2014), Nr. 1, S. 85-93

8. Bäck, J.; Aaltonen, H.; Hellén, H.; Kajos, M. K.; Patokoski, J.; Taipale, R.; Pumpanen, J.; Heinonsalo, J.: Variable emissions of microbial volatile organic compounds (MVOCs) from root-associated fungi isolated from Scots pine. In: Atmos. Environ. 44 (2010), Nr. 30, S. 3651-3659

9. Chitarra, G. S.; Abee, T.; Rombouts, F. M.; Posthumus, M. A.; Dijksterhuis, J.: Germination of Penicillium paneum conidia is regulated by 1-octen-3-ol, a volatile selfinhibitor. In: Appl. Environ. Microbiol. 70 (2004), Nr. 5, S. 2823-2829

10. Combet, E.; Eastwood, D. C.; Burton, K. S.; Henderson, J.: Eight-carbon volatiles in mushrooms and fungi: properties, analysis, and biosynthesis. In: Mycoscience 47 (2006) Nr. 6, S. 317-326 
11. Crutcher, F. K.; Parich, A.; Schuhmacher, R.; Mukherjee, P. K.; Zeilinger, S.; Kenerley, C. M.: A putative terpene cyclase, vir4, is responsible for the biosynthesis of volatile terpene compounds in the biocontrol fungus Trichoderma virens. In: Fungal. Genet. Biol. 56 (2013), S. 67-77

12. Díaz, P.; Señoráns, F. J.; Reglero, G.; Ibañez, E.: Truffle aroma analysis by headspace solid phase microextraction. In: J. Agric. Food Chem. 50 (2002), Nr. 22, S. 64686472

13. Ens, E. J.; Bremner, J. B.; French, K.; Korth, J.: Identification of volatile compounds released by roots of an invasive plant, bitou bush (Chrysanthemoides monilifera spp. rotundata), and their inhibition of native seedling growth. In: Biol. Invasions 11 (2009), Nr. 2, S. 275-287

14. Ezra, D.; Hess, W. M.; Strobel, G. A.: New endophytic isolates of Muscodor albus, a volatile-antibiotic-producing fungus. In: Microbiology 150 (2004), Nr. 12, S. 40234031

15. Farag, M. A.; Ryu, C.-M.; Sumner, L. W.; Paré, P. W.: GC-MS SPME profiling of rhizobacterial volatiles reveals prospective inducers of growth promotion and induced systemic resistance in plants. In: Phytochemistry 67 (2006), Nr. 20, S. 2262-2268

16. Fernando, W. G. D.; Ramarathnam, R.; Krishnamoorthy, A. S.; Savchuk, S. C.: Identification and use of potential bacterial organic antifungal volatiles in biocontrol. In: Soil Biol. Biochem. 37 (2005), Nr. 5, S. 955-964

17. Fäldt, J.; Jonsell, M.; Nordlander, G.; Borg-Karlson, A. K.: Volatiles of bracket fungi Fomitopsis pinicola and Fomes fomentarius and their functions as insect attractants. In: J. Chem. Ecol. 25 (1999), Nr. 3, S. 567-590

18. Gfeller, A.; Laloux, M · Barsics, F.; Kati, D E · Haubruge, E · du Jardin, P · Verheggen, F. J. Lognay, G. Wathelet, J -P · Fauconnier, M.-L.: Characterization of volatile organic compounds emitted by barley (Hordeum vulgare L.) roots and their attractiveness to wireworms. In: J. Chem. Ecol. 39 (2013), Nr. 8, S. 1129-1139

19. Grove, J. F.: Volatile compounds from the mycelium of the mushroom Agraicus bisporus. In: Phytochemistry 20 (1981), Nr. 8, S. 2021-2022

20. Holighaus, G.; Weißbecker, B.; von Fragstein, M.; Schütz, S.: Ubiquitous eight-carbon volatiles of fungi are infochemicals for a specialist fungivore. In: Chemoecology 24 (2014), Nr. 2, S. 57-66

21. Jassbi, A. R.; Zamanizadehnajari, S.; Baldwin, I. T.: Phytotoxic volatiles in the roots and shoots of Artemisia tridentata as detected by headspace solid-phase microextraction and gas chromatographic-mass spectrometry analysis. In: J. Chem. Ecol. 36 (2010), Nr. 12, S. 1398-1407

22. Kaminski, E.; Stawicki, S.; Wasowicz, E.: Volatile flavor compounds produced by molds of Aspergillus, Penicillium and Fungi imperfecti. In: Appl. Environ. Microbiol. 27 (1974), Nr. 6, S. 1001-1004

23. Kleiber, A.; Duan, Q.; Jansen, K.; Junker L. V.; Kammerer, B.; Rennenberg, H.; Ensminger, I.; Gessler, A.; Kreuzwieser, J.: Drought effects on root and needle terpenoid content of a coastal and an interior Douglas fir provenance. In: Tree Physiol. 37 (2017), Nr. 12, S. 1648-1658

24. Lapadatescu, C.; Giniès, C.; Le Quéré, J.-L.; Bonnarme, P.: Novel scheme for biosynthesis of aryl metabolites from L-phenylalanine in the fungus Bjerkandera adusta. In: Appl. Environ. Microbiol. 66 (2000), Nr. 4, S. 1517-1522

25. Lee, B.; Farag, M. A.; Park, H. B.; Kloepper, J. W.; Lee, S. H.; Ryu, C.-M.: Induced resistance by a long-chain bacterial volatile: elicitation of plant systemic defense by a C13 volatile produced by Paenibacillus polymyxa. In: PloS ONE 7 (2012), Nr. 7, S. e48744

26. Lin, C.; Owen, S. M.; Peñuelas, J.: Volatile organic compounds in the roots and rhizosphere of Pinus spp. In: Soil Biol. Biochem. 39 (2007), Nr. 4, S. 951-960

27. Marcetic, M.; Petrovic, S.; Milenkovic, M.; Vujisic, L.; Tesevic, V.; Niketic, M.: Composition and antimicrobial activity of root essential oil of Balkan endemic species Eryngium palmatum. In: Chem. Nat. Compd. 49 (2014), Nr. 6, S. 1140-1142

28. Mauriello, G.; Marino, R.; D’Auria, M.; Cerone, G.; Rana, G. L.: Determination of volatile organic compounds from truffles via SPME-GC-MS. In: J. Chromatogr. Sci. 42 (2004), Nr. 6, S. 299-305

29. Müller, A.; Faubert, P.; Hagen, M.; zu Castell, W.; Polle, A.; Schnitzler, J.-P.; Rosenkranz, M.: Volatile profiles of fungi-Chemotyping of species and ecological functions. In: Fungal Genet. Biol. 54 (2013), S. 25-33

30. Nemčovič, M.; Jakubíková, L.; Víden, I.; Farkaš, V.: Induction of conidiation by endogenous volatile compounds in Trichoderma spp. In: FEMS Microbiol. Lett. 284 (2008), Nr. 2, S. 231-236 
31. Palma, R.; Mutis, A.; Manosalva, R.; Ceballos, R.; Quiroz, A.: Behavioral and electrophysiological responses of Hylastinus obscurus to volatiles released from the roots of Trifolium pratense L. In: J. Soil Sci. Plant Nutr. 12 (2012), Nr. 1, S. 183-193

32. Pelusio, F.; Nilsson, T.; Montanarella, L.; Tilio, R.; Larsen, B.; Madsen, J. Ø.; Facchetti, S.: Headspace solid-phase microextraction analysis of volatile organic sulfur compounds in black and white truffle aroma. In: J. Agric. Food Chem. 43 (1995), Nr. 8, S. 2138-2143.

33. Raal, A.; Arak, E.; Orav, A.; Kailas, T.; Müurisepp, M.: Variation in the composition of the essential oil of commercial Valeriana officinalis L. roots from different countries. In: J. Essent. Oil Res. 20 (2008), Nr. 6, S. 524-529

34. Rostás, M.; Cripps, M. G.; Silcock, P.: Aboveground endophyte affects root volatile emission and host plant selection of a belowground insect. In: Oecologia 177 (2015), Nr. 2, S. 487-497

35. Ryu, C.-M.; Farag, M. A.; Hu, C.-H.; Reddy, M. S.; Wei, H.-X.; Paré, P.W.; Kloepper, J. W.: Bacterial volatiles promote growth in Arabidopsis. In: Proc. Natl. Acad. Sci. U.S.A. 100 (2003), Nr. 8, S. 4927-4932

36. Ryu, C.-M.; Farag, M. A.; Hu, C.-H.; Reddy, M. S.; Kloepper, J. W.; Paré, P. W.: Bacterial volatiles induce systemic resistance in Arabidopsis. In: Plant Physiol. 134 (2004), Nr. 3, S. 1017-1026

37. Schulz, S.; Dickschat, J. S.: Bacterial volatiles: the smell of small organisms. In: Nat. Prod. Rep. 24 (2007), Nr. 4, S. 814-842

38. Splivallo, R.; Bossi, S.; Maffei, M.; Bonfante, P.: Discrimination of truffle fruiting body versus mycelial aromas by stir bar sorptive extraction. In: Phytochemistry 68 (2007), Nr. 20, S. 2584-2598

39. Splivallo, R.; Ottonello, S.; Mello, A.; Karlovsky, P.: Truffle volatiles: from chemical ecology to aroma biosynthesis. In: New Phytol. 189 (2011), Nr. 3, S. 688-699

40. Stotzky, G.; Schenk, S.; Papavizas, G. C.: Volatile organic compounds and microorganisms. In: CRC Crit. Rev. Microbiol. 4 (1976), Nr. 4, S. 333-382

41. Strobel, G. A; Dirkse, E.; Sears, J.; Markworth, C.: Volatile antimicrobials from Muscodor albus, a novel endophytic fungus. In: Mirobiology 147 (2001), Nr. 11; S. 29432950

4hakeow, P.; Weißbecker, B.; Schütz, S.: Volatile organic compounds emitted from fungal-rotting beech. In: Mitt. Dtsch. Ges. Allg. Angew. Ent. 15 (2006), S. 157-160

43. Tirillini, B.; Verdelli, G.; Paolocci, F.; Ciccioli, P.; Frattoni, M.: The volatile organic compounds from the mycelium of Tuber borchii Vitt. In: Phytochemistry 55 (2000), Nr. 8, S. 983-985

44. Weise, T.; Thürmer, A.; Brady, S.; Kai, M.; Daniel, R.; Gottschalk, G.; Piechulla, B.: VOC emission of various Serratia species and isolates and genome analysis of Serratia plymuthica 4Rx13. In: FEMS Microbiol. Lett. 351 (2014), Nr. 1, S. 45-53

45. Wheatley, R. E.; Hackett, C.; Bruce, A.; Kundzewicz, A.: Effect of substrate composition on production and inhibitory activity against wood decay fungi of volatile organic compounds from Trichoderma spp. In: Int. Biodeterior. Biodegradation 39 (1997), S. 199-205.

46. Weissteiner, S.; Schütz, S.: Are different volatile pattern influencing host plant choice of belowground living insects? In: Mitt. Dtsch. Ges. Allg. Angew. Ent. 15 (2006), S. 51-55.

47. Weissteiner, S.; Huetteroth, W.; Kollmann, M.; Weißbecker, B.; Romani, R.; Schachtner, J.; Schütz, S.: Cockchafer larvae smell host root scents in soil. In: PLoS ONE 7 (2012), Nr. 10, S. e45827

48. Zou, C.-S.; Mo, M.-H.; Gu, Y.-Q.; Zhou, J.-P.; Zhang, K.-Q.: Possible contributions of volatile-producing bacteria to soil fungistasis. In: Soil Biol. Biochem. 39 (2007), Nr. 9, S. 2371-2379 


\section{A.5. Apparaturentwicklung für die Untersuchung der} Bodenbeweglichkeit von Wurzel-VOC

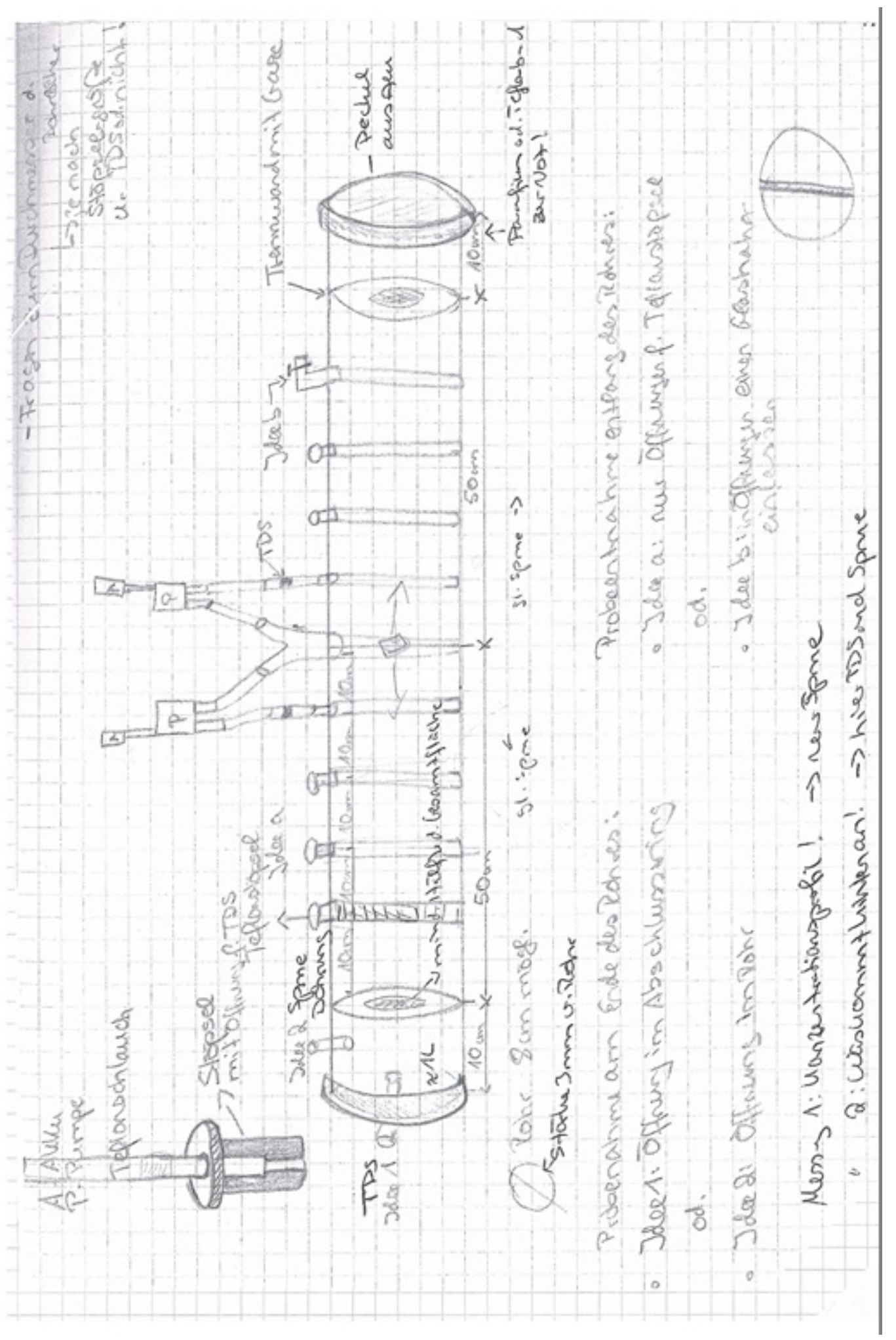

Figure A.2: Handgefertigte Skizze der Apparatur nach ersten Überlegungen 


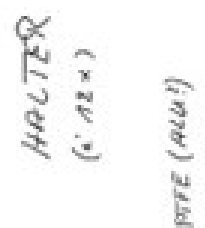
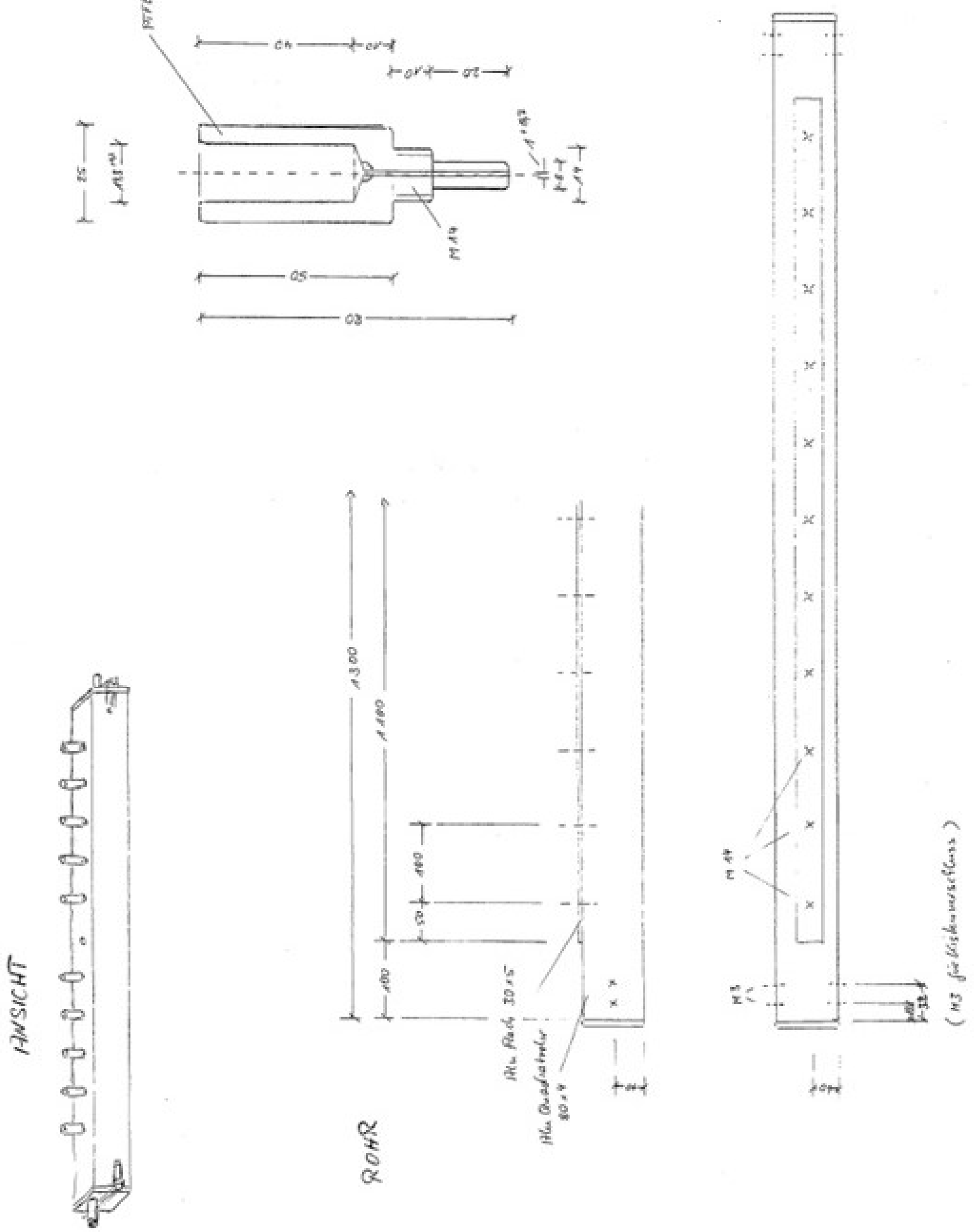

Figure A.3: Technische Zeichung der Apparatur und des Teflonadapters von A. Teichmann 


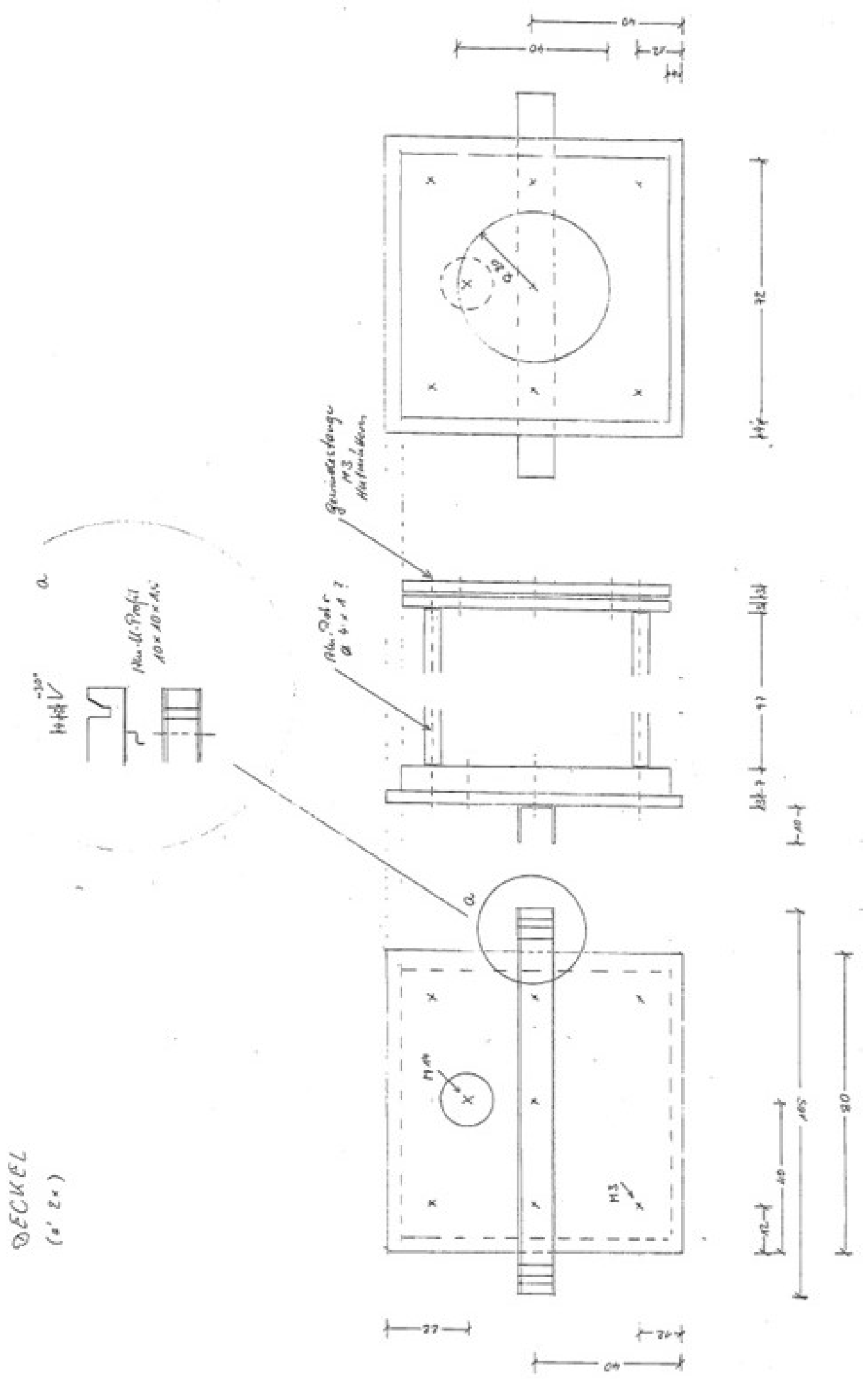

Figure A.4: Technische Zeichnung des Deckels der Apparatur von A. Teichmann 
Table A.10: Messplan des zweiten Hauptversuches mit 3-Octanol: 1. Wiederholung in der Apparatur 1

\begin{tabular}{|c|c|c|c|}
\hline Probenahmzeit [h] & Distanz [cm] & Position & SPME-Faser Nr. \\
\hline $2-4$ & 10 & links & 9 \\
\hline $2-4$ & 20 & links & 10 \\
\hline $2-4$ & 30 & links & 11 \\
\hline $2-4$ & 10 & rechts & 12 \\
\hline $2-4$ & 20 & rechts & 13 \\
\hline $2-4$ & 30 & rechts & 9 \\
\hline $24-26$ & 10 & links & 10 \\
\hline $24-26$ & 20 & links & 11 \\
\hline $24-26$ & 30 & links & 12 \\
\hline $24-26$ & 10 & rechts & 13 \\
\hline $24-26$ & 20 & rechts & 9 \\
\hline $24-26$ & 30 & rechts & 10 \\
\hline $48-50$ & 10 & links & 11 \\
\hline $48-50$ & 20 & links & 12 \\
\hline $48-50$ & 30 & links & 13 \\
\hline $48-50$ & 10 & rechts & 9 \\
\hline $48-50$ & 20 & rechts & 10 \\
\hline $48-50$ & 30 & rechts & 11 \\
\hline $72-74$ & 10 & links & 12 \\
\hline $72-74$ & 20 & links & 13 \\
\hline $72-74$ & 30 & links & 9 \\
\hline $72-74$ & 10 & rechts & 10 \\
\hline $72-74$ & 20 & rechts & 11 \\
\hline $72-74$ & 30 & rechts & 12 \\
\hline 96-98 & 10 & links & 13 \\
\hline $96-98$ & 20 & links & 9 \\
\hline $96-98$ & 30 & links & 10 \\
\hline $96-98$ & 10 & rechts & 11 \\
\hline 96-98 & 20 & rechts & 12 \\
\hline 96-98 & 30 & rechts & 13 \\
\hline
\end{tabular}


Table A.11: Messplan des zweiten Hauptversuches mit 3-Octanol: 2. Wiederholung in der Apparatur 2

\begin{tabular}{|c|c|c|c|}
\hline Probenahmzeit [h] & Distanz [cm] & Position & SPME-Faser Nr. \\
\hline $2-4$ & 10 & links & 9 \\
\hline $2-4$ & 20 & links & 10 \\
\hline $2-4$ & 30 & links & 11 \\
\hline $2-4$ & 10 & rechts & 12 \\
\hline $2-4$ & 20 & rechts & 13 \\
\hline $2-4$ & 30 & rechts & 9 \\
\hline $24-26$ & 10 & links & 10 \\
\hline $24-26$ & 20 & links & 11 \\
\hline $24-26$ & 30 & links & 12 \\
\hline $24-26$ & 10 & rechts & 13 \\
\hline $24-26$ & 20 & rechts & 9 \\
\hline $24-26$ & 30 & rechts & 10 \\
\hline $48-50$ & 10 & links & 11 \\
\hline $48-50$ & 20 & links & 12 \\
\hline $48-50$ & 30 & links & 13 \\
\hline $48-50$ & 10 & rechts & 9 \\
\hline $48-50$ & 20 & rechts & 10 \\
\hline $48-50$ & 30 & rechts & 11 \\
\hline $72-74$ & 10 & links & 12 \\
\hline $72-74$ & 20 & links & 13 \\
\hline $72-74$ & 30 & links & 9 \\
\hline $72-74$ & 10 & rechts & 10 \\
\hline $72-74$ & 20 & rechts & 11 \\
\hline $72-74$ & 30 & rechts & 12 \\
\hline 96-98 & 10 & links & 13 \\
\hline 96-98 & 20 & links & 9 \\
\hline 96-98 & 30 & links & 10 \\
\hline 96-98 & 10 & rechts & 11 \\
\hline 96-98 & 20 & rechts & 12 \\
\hline 96-98 & 30 & rechts & 13 \\
\hline
\end{tabular}

Table A.12: Identifizierte VOC der Kontrollmessungen vom Probesubstrat

\begin{tabular}{|r|l|c|c|c|c|}
\hline $\mathbf{R T}$ & VOC & CAS & $\begin{array}{c}\text { Kontrolle } \\
(0-60 \text { min })\end{array}$ & $\begin{array}{c}\text { Kontrolle } \\
(90-150 \mathrm{~min})\end{array}$ & $\begin{array}{c}\text { Kontrolle } \\
(180-240 \mathrm{~min})\end{array}$ \\
\hline 8,474 & Camphen & $000079-92-5$ & 1 & 1 & 1 \\
\hline 10,097 & 3 -Caren & $013466-78-9$ & 1 & 1 & 1 \\
\hline 10,606 & Cymol & $000099-87-6$ & 1 & 1 & 1 \\
\hline 14,892 & Dodecan & $000112-40-3$ & 1 & 0 & 1 \\
\hline 19,141 & $\alpha$-Cedren & $000469-61-4$ & 1 & 1 & 1 \\
\hline 19,325 & Isolongifolen & $001135-66-6$ & 1 & 1 & 1 \\
\hline 19,841 & Cedr-9-en & $021996-77-0$ & 1 & 1 & 1 \\
\hline 20,158 & $\beta$-Patchoulen & $000514-51-2$ & 1 & 1 & 1 \\
\hline
\end{tabular}

$$
\mathrm{RT}=\text { Retentionszeit }
$$


Table A.13: Identifizierte VOC der Kontrollmessungen von der leeren Apparatur

\begin{tabular}{|c|c|r|l|c|}
\hline Probepostion & Messzeit [h] & RT & VOC & CAS \\
\hline Apparatur Mitte & 2 & 7,427 & Heptanal & $000111-71-7$ \\
\cline { 2 - 5 } & & 9,994 & Octanal & $000124-13-0$ \\
\cline { 2 - 5 } & & 12,553 & Nonanal & $000124-19-6$ \\
\hline Apparatur links & 2 & 7,478 & Heptanal & $000111-71-7$ \\
\cline { 2 - 5 } & & 10,023 & Octanal & $000124-13-0$ \\
\cline { 2 - 5 } & & 12,561 & Nonanal & $000124-19-6$ \\
\cline { 2 - 5 } & & 13,778 & Benzeneethanamin & $040629-66-1$ \\
\hline Apparatur rechts & 2 & 7,493 & Heptanal & $000111-71-7$ \\
\cline { 2 - 5 } & & 10,008 & Octanal & $000124-13-0$ \\
\cline { 2 - 5 } & & 12,575 & Nonanal & $000124-19-6$ \\
\cline { 2 - 5 } & & 21,479 & Pentasiloxan & $000141-63-9$ \\
\hline
\end{tabular}

$\mathrm{RT}=$ Retentionszeit

Table A.14: Identifizierte VOC der Kontrollmessungen von der Apparatur mit eingefülltem Probesubstrat

\begin{tabular}{|c|c|r|l|c|}
\hline Probepostion & Messzeit [h] & RT & VOC & CAS \\
\hline Apparatur Mitte & 2 & 12,789 & $?$ & \\
\cline { 2 - 5 } & & 13,778 & Benzeneethanamin & $040629-66-1$ \\
\cline { 2 - 5 } & & 17,82 & $?$ & \\
\cline { 2 - 5 } & & 21,479 & $?$ & \\
\hline Apparatur links & 2 & 10,134 & Octanal & $000124-13-0$ \\
\cline { 2 - 5 } & & 11,506 & 1 -Chloroundecan & $002473-03-2$ \\
\cline { 2 - 5 } & & 12,612 & Nonanal & $000124-19-6$ \\
\cline { 2 - 5 } & & 13,778 & Benzeneethanamin & $040629-66-1$ \\
\cline { 2 - 5 } & & 19,723 & (+)-Longifolen & $000475-20-7$ \\
\hline Apparatur rechts & & 10,031 & Octanal & $000124-13-0$ \\
\cline { 2 - 5 } & & 11,506 & $1-$ Chlorooctan & $000111-85-3$ \\
\cline { 2 - 5 } & & 12,561 & Nonanal & $000124-19-6$ \\
\cline { 2 - 5 } & & 13,763 & $?$ & \\
\cline { 2 - 5 } & & 17,813 & $?$ & \\
\cline { 2 - 5 } & & & & \\
\end{tabular}

$\mathrm{RT}=$ Retentionszeit,$?=$ nicht identifiziertes VOC 
Table A.15: Peakfläche (SIM: Ion 43) zu den Untersuchungen mit 3-Octanon bei $10 \mathrm{~cm}$ links und rechts von der Startposition

\begin{tabular}{|r|r|r|l|r|}
\hline Messzeit [h] & Distanz [cm] & Peakfläche (Ion 43) & Position & SPME Faser Nr. \\
\hline $2-4$ & 10 & 0 & rechts & 6 \\
\hline $4-6$ & 10 & 0 & rechts & 6 \\
\hline $6-8$ & 10 & 117.099 .672 & rechts & 6 \\
\hline $94-96$ & 10 & 194.517 .499 & rechts & 10 \\
\hline $96-98$ & 10 & 185.377 .306 & rechts & 10 \\
\hline $98-100$ & 10 & 344.992 .100 & rechts & 10 \\
\hline $2-4$ & 10 & 0 & links & 10 \\
\hline $4-6$ & 10 & 0 & links & 10 \\
\hline $6-8$ & 10 & 0 & links & 10 \\
\hline $94-96$ & 10 & 108.043 .772 & links & 6 \\
\hline $96-98$ & 10 & 105.865 .401 & links & 6 \\
\hline $98-100$ & 10 & 174.171 .327 & links & \\
\hline
\end{tabular}

Table A.16: Peakfläche (SIM: Ion 43) zu den Untersuchungen mit 3-Octanon bei den Distanzen 10, 30 und $50 \mathrm{~cm}$ rechts von der Startposition

\begin{tabular}{|r|r|r|r|}
\hline Messzeit [h] & Distanz [cm] & Peakfläche (Ion 43) & SPME Faser Nr. \\
\hline $120-122$ & 10 & 165.779 .576 & 6 \\
\hline $120-122$ & 30 & 22.122 .597 & 9 \\
\hline $120-122$ & 50 & 566.780 & 10 \\
\hline $146-148$ & 10 & 293.286 & 6 \\
\hline $146-148$ & 30 & 30.243 .466 & 9 \\
\hline $146-148$ & 50 & 170.721 .350 & 10 \\
\hline $256-258$ & 10 & 129.884 .988 & 6 \\
\hline $256-258$ & 30 & 63.792 .102 & 9 \\
\hline $256-258$ & 50 & 57.215 & 10 \\
\hline $276-278$ & 10 & 124.267 .859 & 6 \\
\hline $276-278$ & 30 & 60.720 .759 & 9 \\
\hline $276-278$ & 50 & 0 & 6 \\
\hline $976-978$ & 10 & 10.587 .360 & 9 \\
\hline $976-978$ & 30 & 6.315 .238 & 10 \\
\hline $976-978$ & 50 & 0 & \\
\hline
\end{tabular}


Table A.17: Peakflächen von 3-Octanon (SIM: Ion 43) für den Hauptversuch Teil 2 1. Wiederholung: 3-Octanon

\begin{tabular}{|c|c|c|c|}
\hline \multicolumn{4}{|c|}{ 1. Wiederholung: 3-Octanon } \\
\hline Messzeit [h] & Distanz [cm] & Peakfläche in Mio. & \% vom Vergleichswert \\
\hline $2-4$ & 10 & 0 & 0 \\
\hline $2-4$ & 20 & 0 & 0 \\
\hline $2-4$ & 30 & 0 & 0 \\
\hline $2-4$ & 40 & 0 & 0 \\
\hline $2-4$ & 50 & 0 & 0 \\
\hline $24-26$ & 10 & 1.428 .069 & 0,06 \\
\hline $24-26$ & 20 & 0 & 0 \\
\hline $24-26$ & 30 & 0 & 0 \\
\hline $24-26$ & 40 & 0 & 0 \\
\hline $24-26$ & 50 & 0 & 0 \\
\hline $48-50$ & 10 & 5.285 .797 & 0,24 \\
\hline $48-50$ & 20 & 4.490 .174 & 0,20 \\
\hline $48-50$ & 30 & 764.517 & 0,03 \\
\hline $48-50$ & 40 & 0 & 0 \\
\hline $48-50$ & 50 & 0 & 0 \\
\hline $72-74$ & 10 & 2.906 .711 & 0,13 \\
\hline $72-74$ & 20 & 4.053 .582 & 0,18 \\
\hline $72-74$ & 30 & 2.290 .816 & 0,10 \\
\hline $72-74$ & 40 & 0 & 0 \\
\hline $72-74$ & 50 & 0 & 0 \\
\hline $96-98$ & 10 & 4.254 .199 & 0,19 \\
\hline $96-98$ & 20 & 3.790 .332 & 0,17 \\
\hline 96-98 & 30 & 3.631 .023 & 0,16 \\
\hline 96-98 & 40 & 0 & 0 \\
\hline $96-98$ & 50 & 0 & 0 \\
\hline
\end{tabular}

\begin{tabular}{|r|r|r|r|}
\hline \hline & 2. Wiederholung: 3-Octanon & \\
\hline $2-4$ & 10 & 155.102 .827 & 6,91 \\
\hline $2-4$ & 20 & 4.182 .849 & 0,19 \\
\hline $2-4$ & 30 & 577.548 & 0,03 \\
\hline $2-4$ & 40 & 0 & 0 \\
\hline $2-4$ & 50 & 0 & 0 \\
\hline $24-26$ & 10 & 310.385 .775 & 13,83 \\
\hline $24-26$ & 20 & 237.801 .304 & 10,60 \\
\hline $24-26$ & 30 & 17.618 .306 & 0,79 \\
\hline $24-26$ & 40 & 0 & 0 \\
\hline $24-26$ & 50 & 0 & 0 \\
\hline $48-50$ & 10 & 275.153 .810 & 12,26 \\
\hline $48-50$ & 20 & 226.684 .581 & 10,10 \\
\hline $48-50$ & 30 & 62.425 .960 & 2,78 \\
\hline $48-50$ & 40 & 0 & 0 \\
\hline $48-50$ & 50 & 0 & 0 \\
\hline $72-74$ & 10 & 237.322 .202 & 0 \\
\hline $72-74$ & 20 & 203.391 .669 & 0 \\
\hline $72-74$ & 30 & 86.153 .785 & 3,84 \\
\hline $72-74$ & 40 & 1.560 .029 & 0,07 \\
\hline $72-74$ & 50 & 0 & 0 \\
\hline $96-98$ & 10 & 210.289 .658 & 9,37 \\
\hline $96-98$ & 20 & 201.750 .044 & 0,99 \\
\hline $96-98$ & 30 & 103.891 .912 & 034 \\
\hline $96-98$ & 40 & 7.674 .949 & 0 \\
\hline $96-98$ & 50 & & 0 \\
\hline & & 0 & 0 \\
\hline
\end{tabular}

